\title{
Overview of antimonide based III-V semiconductor epitaxial layers and their applications at the center for quantum devices
}

\author{
M. Razeghi ${ }^{\mathrm{a}}$ \\ Center for Quantum Devices, Northwestern University, Evanston, IL 60208, USA
}

Received: 11 March 2003 / Received in final from: 22 April 2003 / Accepted: 26 June 2003

Published online: 15 September 2003 - (C) EDP Sciences

\begin{abstract}
The properties of Sb-based III-V semiconductor compounds for optoelectronic applications in the mid-wavelength infrared (MWIR) and long-wavelength infrared (LWIR) range were reviewed. The growths of the Sb-based binary, ternary and quaternary were studied by molecular beam epitaxy (MBE) and metalorganic chemical vapor deposition (MOCVD). The structural, optical and electrical characterizations were carried out. Focal plane array, photoconductors and photodiodes were fabricated for the MWIR and LWIR range. Doublehetero structure (DH), multi-quantum well (MQW) and strained superlattice (SSL) lasers in the 3-5 $\mu \mathrm{m}$ range were fabricated. InAs-GaSb type-II superlattices were designed, grown and fabricated into photodetectors for the MWIR and LWIR range.
\end{abstract}

PACS. 71.20.Nr Semiconductor compounds - 71.55.Eq III-V semiconductors - 73.21.Cd Superlattices

\section{Introduction}

Techniques for infrared (IR) detection and emission have been advanced a long way since the first discovery of infrared radiation by William Herschel in 1800. Since objects at temperatures between 0 and $1000{ }^{\circ} \mathrm{C}$ emit most of their energy as radiation with an infrared wavelength $1<\lambda<20 \mu \mathrm{m}$ (Fig. 1.1) and the atmosphere has the transmission widow at $3-5 \mu \mathrm{m}$ (mid-wavelength infrared, MWIR) and 8-14 $\mu \mathrm{m}$ (long-wavelength infrared, LWIR) region (Fig. 1.2), infrared detectors and lasers at these wavelength are of great importance for various applications. On the other hand, the $5-8 \mu \mathrm{m}$ regions also finds applications in the proximity fuzes where high atmospheric absorption is important to detect the target only in the close proximity. Infrared detectors in the very long wavelength range (VLWIR) have applications such as space based astronomy and early detection of long-range missiles.

$\mathrm{HgCdTe}(\mathrm{MCT})$ is a well established material system which has been the dominant system for mid and long-wavelength IR photodetectors. However, MCT suffers from instability and non-uniformity problems over larger area due to the high $\mathrm{Hg}$ vapor pressure. This has intensified the search for alternative infrared material system. Sb based materials including III-Sb binary and its ternary and quaternary alloys are suitable candidates for the applications in the infrared range. Figure 1.3 shows the bandgap vs. lattice constant diagram of III-V alloys. InSb

\footnotetext{
a e-mail: razeghi@ece.northwestern.edu
}

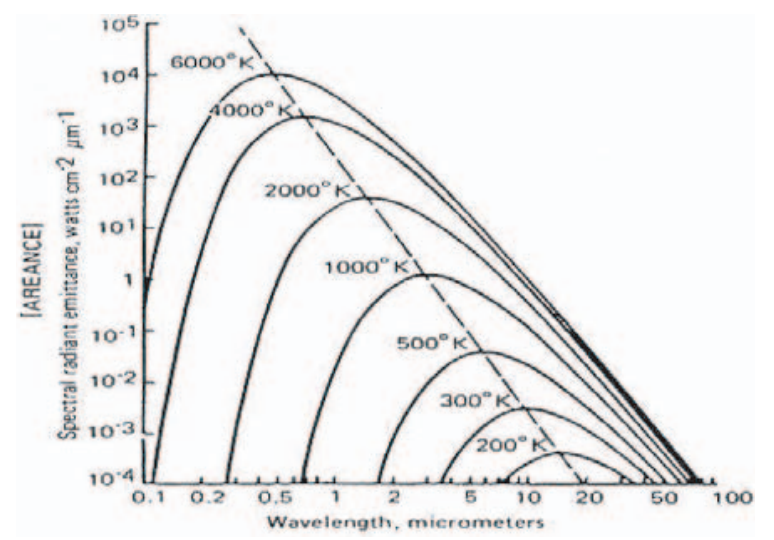

Fig. 1.1. Radiant emittance of a blackbody versus blackbody temperature [1].

has band gap energy of $0.18 \mathrm{eV}$ at room temperature and $0.23 \mathrm{eV}$ at $77 \mathrm{~K}$, enabling it to cover the entire MWIR region up to $5.5 \mu \mathrm{m}$. For longer wavelength, arsenic can be added into InSb to form InAsSb, which shows reduced band gap energy. As an alternative to the InAsSb system, InTlSb is also a potential material for $\lambda>8 \mu \mathrm{m}$ detectors. Besides adding As or $\mathrm{Tl}$ to an InSb lattice, it is also possible to substitute $\mathrm{Bi}$. As a result, a rapid reduction of the bandgap is expected. However, the large miscibility gap limits $\mathrm{Tl}$ and $\mathrm{Bi}$ incorporation to high composition and thus further reduction of band gap. In order to exceed the limitations imposed by the miscibility gap, arsenic can be 


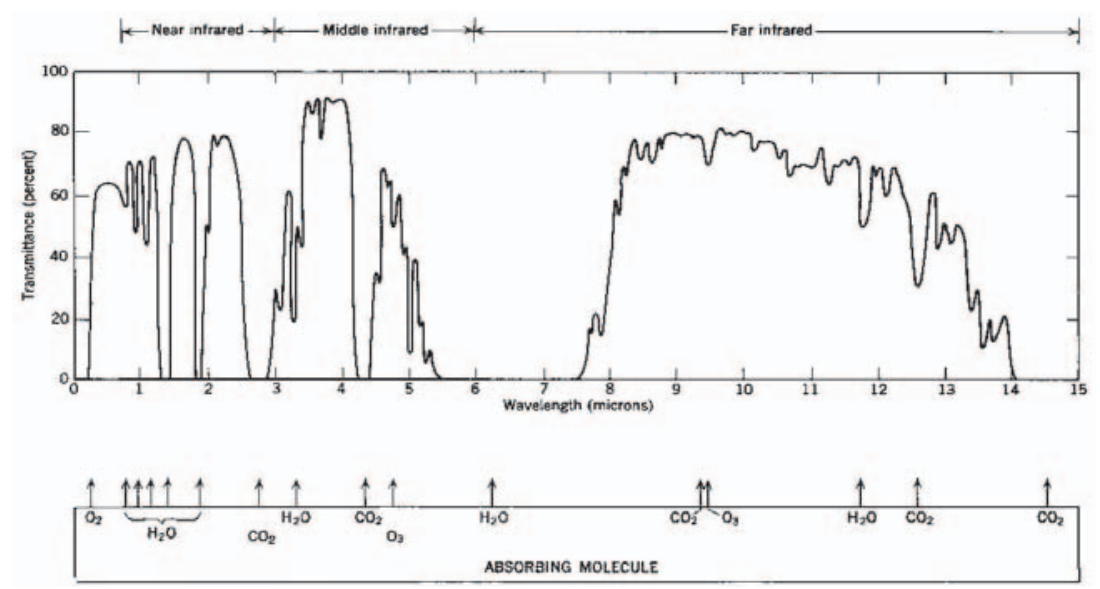

Fig. 1.2. Transmittance of the atmosphere for a 6000 feet horizontal path at ea level.

added to InTlSb to form a quaternary material with an extended photoresponse cutoff wavelength.

The development of laser source for MWIR $(3-5 \mu \mathrm{m})$ has been particularly difficult because of lack of suitable high-quality heterostructure semiconductor materials. InAsSb/InAsSbP is one of the new material systems that have good carrier and optical confinement with good lattice-matching, and thus very promising for laser diode fabrication. Double heterostructure (DH), multiple quantum well (MQW), and superlattice structure lasers using InAsSbP alloy system can cover $3-5 \mu \mathrm{m}$ region by adjusting its alloy composition.

Recently, type-II superlattices have been proposed as an alternative to $\mathrm{HgCdTe}$ for the strategic windows of $3-5 \mu \mathrm{m}$ and $8-12 \mu \mathrm{m}$ as well as longer wavelengths. Unlike HgCdTe, type-II superlattices are constructed from the III-V material system, and hence they have much better mechanical properties and material uniformity. The electron effective mass in these superlattices is higher than the electron effective mass in $\mathrm{HgCdTe}$ and InAsSb, therefore the tunneling current is less. The bandgap of the superlattice can be changed to cover a wide IR range (from $\sim 2 \mu \mathrm{m}$ to above $25 \mu \mathrm{m}$ ) by changing the thickness of the layers rather than the material composition. Also, in comparison to quantum well infrared photodetectors (QWIPs), type-II superlattices have much higher quantum efficiency while they do not require surface gratings for absorption of normal incident light.

\section{Antimony}

Antimony (symbol: $\mathrm{Sb}$ ) is a semimetal, which has an atomic number of 51 and atomic weight of 121.760. It is located in the group 15 (VA) and period 5 in the chemical period table. Antimony is solid at room temperature and standard state. Antimony in its elemental form is a silvery white, brittle crystalline solid. It is not acted on by air at room temperature, but burns brilliantly when heated with the formation of white fumes. It is a poor conductor of heat and electricity. The physical data of antimony are summarized in Table 2.1.

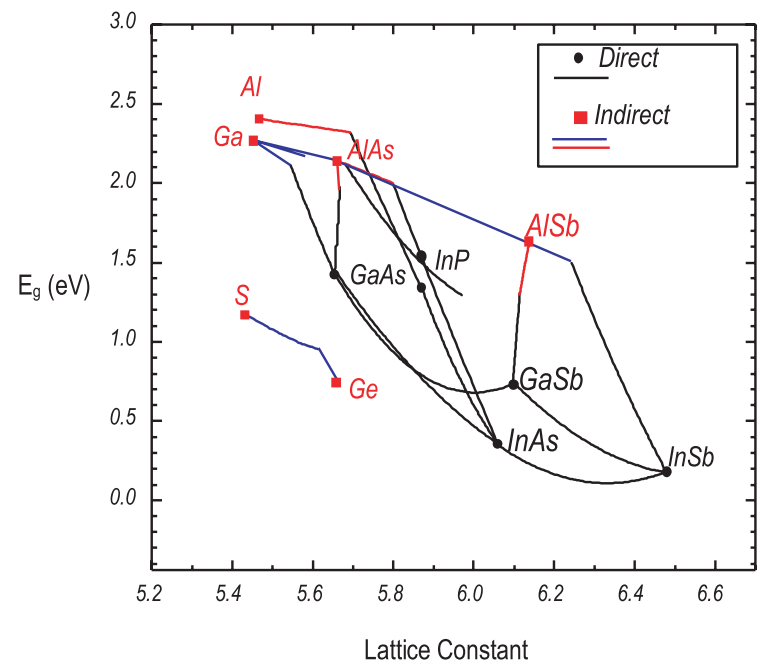

Fig. 1.3. The bandgap vs. lattice constant diagram of III-V alloys.

Antimony has been known and used since the ancient time. The name antimony comes from Greek words "anti + monos" meaning "not alone", since it's often found in ores such as stibnite and valentines. The origin of the symbol Sb comes from the Latin word "stibium" or mark.

Upon heating, antimony reacts with oxygen in air to form the trioxide antimony (III) oxide, $\mathrm{Sb}_{2} \mathrm{O}_{3}$.

$$
4 \mathrm{Sb}(\mathrm{s})+3 \mathrm{O}_{2}(\mathrm{~g}) \rightarrow 2 \mathrm{Sb}_{2} \mathrm{O}_{3}(\mathrm{~s})
$$

At red heat, antimony reacts with water to form the trioxide antimony (III) oxide, $\mathrm{Sb}_{2} \mathrm{O}_{3}$. Antimony reacts more slowly at ambient temperatures.

$$
2 \mathrm{Sb}(\mathrm{s})+3 \mathrm{H}_{2} \mathrm{O}(\mathrm{g}) \rightarrow \mathrm{Sb}_{2} \mathrm{O}_{3}(\mathrm{~s})+3 \mathrm{H}_{2}(\mathrm{~g})
$$

Antimony reacts under controlled conditions with the halogens fluorine, $\mathrm{F}_{2}$, chlorine, $\mathrm{Cl}_{2}$, bromine, $\mathrm{Br}_{2}$, and iodine, $\mathrm{I}_{2}$, to form the respective trihalides antimony (III) fluoride, $\mathrm{SbF}_{3}$, antimony (III) chloride, 
Table 2.1. Summary of the physical data of antimony.

\begin{tabular}{c|c|c}
\hline & Symbol & $\mathrm{Sb}$ \\
\hline \multirow{4}{*}{ Steric } & Atomic number & 51 \\
\cline { 2 - 3 } & Atomic weight & $121.760 \mathrm{amu}$ \\
\cline { 2 - 3 } & Atomic radius & $1.53 \AA$ \\
\cline { 2 - 3 } & Ionic radius & $0.76 \AA$ \\
\cline { 2 - 3 } & Covalent radius & $1.40 \AA$ \\
\cline { 2 - 3 } & Atomic volume & $18.23 \mathrm{~cm}^{3} / \mathrm{mol}^{3}$ \\
\cline { 2 - 3 } & Density $(293 \mathrm{~K})$ & $6.684 \mathrm{~g} / \mathrm{cm}^{3}$ \\
\hline Electronic & Crystal structure & $\mathrm{Rhombohedral}$ \\
\cline { 2 - 3 } & Shells & $2,8,18,18,5$ \\
\cline { 2 - 3 } & Orbitals & {$[\mathrm{Kr}] 4 d^{10} 5 \mathrm{~s}^{2} 5 p^{3}$} \\
\cline { 2 - 3 } & Electronegativity & $1.8,1.9$ \\
\cline { 2 - 3 } & Oxidation states & $5,3,-3$ \\
\hline Thermal & Electrical resistivity & $4 \times 10^{-7} \mathrm{ohm} \mathrm{m}$ \\
\cline { 2 - 3 } & Melting point & $630.74{ }^{\circ} \mathrm{C}$ \\
\cline { 2 - 3 } & Boiling point & $1587{ }^{\circ} \mathrm{C}$ \\
\cline { 2 - 3 } & Specific heat & $0.21 \mathrm{~J} / \mathrm{g} \mathrm{K}$ \\
\cline { 2 - 3 } & Heat of fusion & $19.870 \mathrm{~kJ} / \mathrm{mol}$ \\
\cline { 2 - 3 } & Heat of vaporization & $77.140 \mathrm{~kJ} / \mathrm{mol}$ \\
\cline { 2 - 3 } & Thermal conductivity & $0.243 \mathrm{~W} / \mathrm{cm} \mathrm{K}$ \\
\hline
\end{tabular}

$\mathrm{SbCl}_{3}$, antimony (III) bromide, $\mathrm{SbBr}_{3}$, and antimony (III) iodide, $\mathrm{SbI}_{3}$.

$$
\begin{aligned}
& 2 \mathrm{Sb}(\mathrm{s})+3 \mathrm{~F}_{2}(\mathrm{~g}) \rightarrow 2 \mathrm{SbF}_{3}(\mathrm{~s}) \\
& 2 \mathrm{Sb}(\mathrm{s})+3 \mathrm{Cl}_{2}(\mathrm{~g}) \rightarrow 2 \mathrm{SbCl}_{3}(\mathrm{~s}) \\
& 2 \mathrm{Sb}(\mathrm{s})+3 \mathrm{Br}_{2}(\mathrm{~g}) \rightarrow 2 \mathrm{SbBr}_{3}(\mathrm{~s}) \\
& 2 \mathrm{Sb}(\mathrm{s})+3 \mathrm{I}_{2}(\mathrm{~g}) \rightarrow 2 \mathrm{SbI}_{3}(\mathrm{~s})
\end{aligned}
$$

Antimony is finding use in semiconductor technology for making infrared detectors, diodes and Hall-effect devices. Antimony is alloyed with lead to increase lead's durability. Antimony alloys are also used in batteries, low friction metals, type metal and cable sheathing, among other products. Antimony compounds are used to make flameproofing materials, paints, ceramic enamels, glass and pottery. A few kinds of over-the-counter cold and flu remedies use antimony compounds.

\section{$3 \operatorname{lnSb}$}

\subsection{Introduction}

$\mathrm{InSb}$ is a direct small energy gap semiconductor whose semiconductor properties were first reported by Welker in 1952 [2]. It was soon discovered that InSb had the smallest energy gap among any semiconductors known at that time and naturally its application as infrared detector became obvious. The bandgap energy of $0.18 \mathrm{eV}$ at room temperature indicated it would have a long wavelength limit of approximately $7 \mu \mathrm{m}$ and when cooled with liquid nitrogen the gap increased to $0.23 \mathrm{eV}$, enabling it to

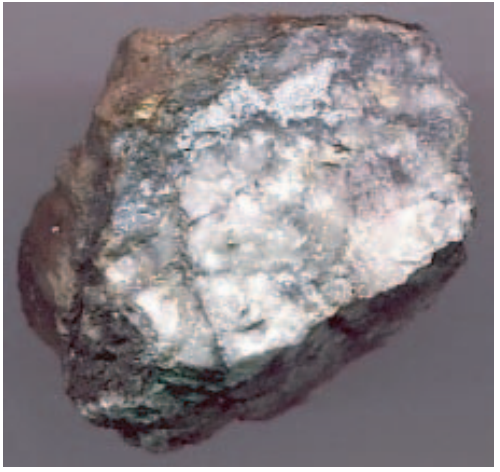

Fig. 2.1. Silvery-white native antimony with stibnite in quartz.

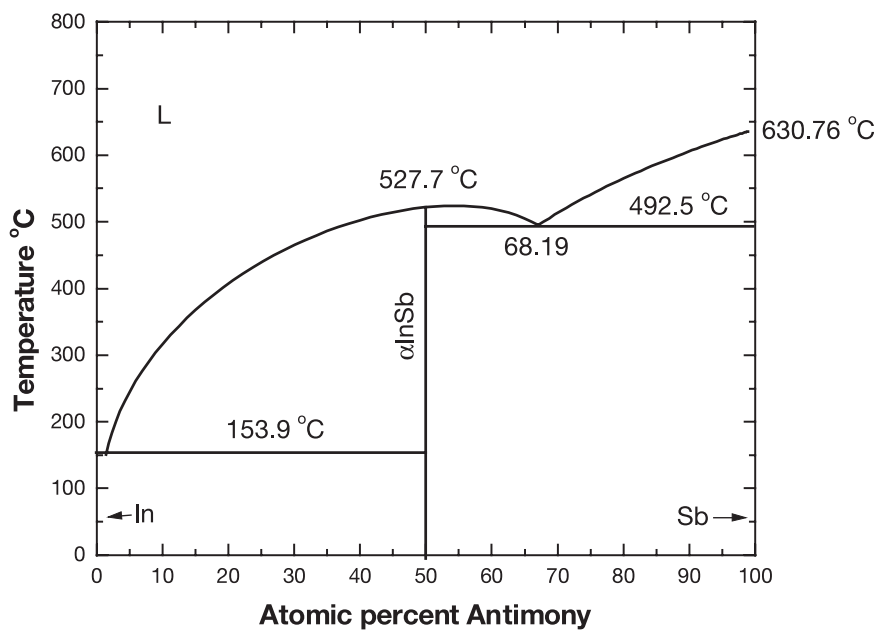

Fig. 3.1. Temperature-composition binary phase diagram of In-Sb, describing the equilibrium of solid with a liquid InSb.

cover the entire MWIR up to $5.5 \mu \mathrm{m}$. Furthermore, it is a relatively robust material with high quantum absorption efficiency and sensitivity in the MWIR. The interest in InSb stemmed not only from its small energy gap but also from the fact that it could be prepared in high quality single crystal form. As shown in the phase diagram of Figure 3.1 [3], InSb exhibits a congruent melting point at $525.7^{\circ} \mathrm{C}$, allowing it to be grown from the melt by the conventional technique. Today, 2 and 3 inch $\mathrm{InSb}$ substrates are commercially available.

Material parameters and characteristics of bulk InSb have been investigated and refined over the years. The experimental bandgap, $E_{0}$, and intrinsic carrier concentration $n_{i}$, have been measured as a function of temperature and the following expressions [4] have been found to agree well with the experimental data:

$$
\begin{gathered}
E_{0}(T)=\left[0.235-\frac{3.2 \times 10^{-4} T^{2}}{(220+T)}\right] \mathrm{eV} \\
n_{i}=5.63 \times 10^{14} T^{3 / 2} \exp \left(-0.127 / k_{\mathrm{B}} T\right) \mathrm{cm}^{-3} .
\end{gathered}
$$

Other important parameters such as lattice constant, electron and hole masses, refractive index and thermal expansion coefficient are summarized in Table 3.1 [5]. 
Table 3.1. Physical properties of InSb.

\begin{tabular}{lcc}
\hline & $T(\mathrm{~K})$ & $\mathrm{InSb}$ \\
\hline Crystal structure & & $\mathrm{Cub}(\mathrm{ZnS})$ \\
\hline Lattice constant $(\AA)$ & 300 & 6.47877 \\
\hline Coef. of thermal expansion & 300 & 5.04 \\
$\left(10^{-6} \mathrm{~K}^{-1}\right)$ & 80 & 6.50 \\
\hline Density $\gamma\left(\mathrm{g} / \mathrm{cm}^{3}\right)$ & 300 & 5.7751 \\
\hline Melting point $(\mathrm{K})$ & & 803 \\
\hline Energy gap $(\mathrm{eV})$ & 4.2 & 0.2357 \\
& 80 & 0.228 \\
& 300 & 0.180 \\
\hline Thermal coefficient of $E_{\mathrm{g}}$ & $100-300$ & $-2.8 \times 10^{4}$ \\
\hline$m_{e}^{*} / m_{0}$ & 4.2 & 0.0145 \\
& 300 & 0.0116 \\
\hline$m_{l h}^{*} / m_{0}$ & 4.2 & 0.0149 \\
\hline$m_{h h}^{*} / m_{0}$ & 4.2 & 0.41 \\
\hline Momentum matrix element $P$ & & $9.4 \times 10^{-8}$ \\
$\left(\mathrm{~cm}{ }^{2} / \mathrm{V}\right.$ s) & & \\
\hline Electron mobility $\left(\mathrm{cm}^{2} / \mathrm{V} \mathrm{s}\right)$ & 300 & $8 \times 10^{4}$ \\
& 77 & $10^{6}$ \\
\hline Hole mobility $\left(\mathrm{cm}^{2} / \mathrm{V} \mathrm{s}\right)$ & 300 & 800 \\
\hline Intrinsic carrier conc. $\left(\mathrm{cm}{ }^{-3}\right)$ & 77 & $10^{4}$ \\
\hline Static dielectric constant & 200 & $2.6 \times 10^{9}$ \\
\hline Optical phonon $\left(\mathrm{cm}^{-1}\right)$ & 300 & $1.9 \times 10^{14}$ \\
\hline TO & & 3.96 \\
\hline
\end{tabular}

InSb has been utilized for many purposes due to its properties described above. Because of the low electron effective mass and the resulting large electron mobility, InSb can be used for very high speed electronic devices and is being used in magnetoresistive sensors in the automotive industry. High performance InSb detectors have been fabricated with this material for decades $[6,7]$. The InSb detector arrays are $p-n$ junctions made mostly by implanting $\mathrm{Be}^{+}$into $n$-type InSb substrate. Such processes yield high quality $p$ - $n$ junctions with the low leakage currents and the high breakdown voltage, which are desirable characteristics for detector arrays. Surface passivation is carried out to minimize the surface leakage current, which can be significant in small-sized detectors (pixels) in high spatial resolution arrays. Surface passivation should exhibit excellent adhesion and must be mechanically stable enough to allow bonding after metallization. Since anodically grown native oxide suffers from high surface state density, $\mathrm{SiO}_{x}$ dielectric layer is normally preferred as the surface passivation.
A fully monolithic structure, where all optical detection and signal processing functions are performed on the same material, is the most ideal architecture. Such monolithic structures have been reported using InSb metaloxide semiconductor field effect transistor [8] but the low breakdown voltage in narrow bandgap material have led to low charge handling capabilities. Additionally, the serious problems involving noise and charge trapping in narrowgap semiconductor reduce charge transfer efficiency. These disadvantages have limited developments of a fully monolithic structure using a narrow-gap material.

An alternative method is the hybrid integration approach where the photon detection process is performed by the narrow bandgap detector array and the signal is transferred to a Si-based charge storage and multiplexed readout. Indium bump is normally used as the interconnect between the optical and the electrical components. Current manufacturing device processes require that bulk materials to be thinned before or after hybridization to achieve backside illumination with reasonable quantum efficiency and minimum crosstalk. The advantage of the hybrid architecture is that the performances of two different devices can be separately optimized. However, this structure suffers from the difference in the thermal expansion coefficients between detector and the multiplexer materials. In addition, the substrate thinning process is a very delicate process that can lower the yield and the reproducibility of the detectors.

The best solution is the pseudo-monolithic integration architecture where InSb detectors can be integrated with GaAs- or Si-based electronic readouts on a common substrate. This would require the ability to grow and fabricate InSb detectors on GaAs or Si. Advantages of such integration are three-fold. First, there back-illumination detector structures will not suffer from having to thin the substrate since it is transparent in the $3-5 \mu \mathrm{m}$ range. Second, the integration eliminates the indium bump process. Third, larger array can be realized since larger (greater than 3 inch InSb substrate that are available) substrate are available in GaAs or Si. Therefore, successful pseudomonolithic integration will permit the production of larger reliable focal plan arrays.

\subsection{MOCVD growth of InSb}

\subsubsection{Material growth and characterization}

The growth of InSb was performed on InSb, GaAs and GaAs-coated silicon substrates with a low pressure MOCVD. TMIn, TEIn, and TMSb were used as precursors. The growth conditions were studied using TMIn and TMSb by varying the growth temperature and V/III ratio. Table 3.2 summarizes the growth parameters and conditions. The low melting temperature of $\operatorname{InSb}\left(525{ }^{\circ} \mathrm{C}\right)$ requires low growth temperature, typically not greater than $475{ }^{\circ} \mathrm{C}$, to prevent surface deterioration. At these growth temperatures, TMIn and TMSb decompose at a reasonable rate. The growth rate of InSb films was approximately 
Table 3.2. Growth parameters and conditions used for the deposition of normally undoped InSb epilayers.

\begin{tabular}{c|c|c}
\hline \multicolumn{2}{c|}{ Growth temperature } & $425{ }^{\circ} \mathrm{C}-475{ }^{\circ} \mathrm{C}$ \\
\hline \multicolumn{2}{c}{ Growth pressure } & 76 torr \\
\hline Substrate orientation & InSb, GaAs, GaAs-coated Si \\
\hline \multirow{2}{*}{ TMIn } & Temperature & $18{ }^{\circ} \mathrm{C}$ \\
\cline { 2 - 3 } & Flow rate & $\sim 50 \mathrm{sccm}$ \\
\hline \multirow{2}{*}{ TMSb } & Temperature & $0{ }^{\circ} \mathrm{C}$ \\
\cline { 2 - 3 } & Flow rate & $16-36 \mathrm{sccm}$ \\
\hline $\mathrm{H}_{2}$ & Flow rate & $1.5 \mathrm{l} / \mathrm{min}$ \\
\hline
\end{tabular}

$1 \mu \mathrm{m}$ per hour and the film thickness ranged from $0.7 \mu \mathrm{m}$ to $6.3 \mu \mathrm{m}$.

TEIn was also tried as alternate In source for InSb growth. Since decomposition of TEIn is complete at the investigated growth temperatures, it was expected to yield more uniform growth over the susceptor area where a small temperature gradient could exit. The growth conditions using TEIn was similar to those in Table 3.2 except that the flow rate of TEIn was adjusted so that similar $\mathrm{V} / \mathrm{III}$ ratio range could be explored.

The substrates used for the growth were $\mathrm{InSb}$ and GaAs substrates, directed $2^{\circ}$ off the (100) toward (110) direction. The InSb substrates were Te-doped to a concentration of about $1 \times 10^{18} \mathrm{~cm}^{-3}$. The GaAs substrates were from Sumitomo as semi-insulating (Cr-doped) and superclean. The $n^{+}$-GaAs and GaAs coated Si substrates were superclean and supplied by Hitachi cable Ltd. and Kopin Corporation, respectively.

\subsubsection{Structure characteristics}

At first a series of samples were investigated using TEIn as the In source. TEIn has a weaker hydrocarbon bond than TMIn and has a decomposition temperature as low as $40{ }^{\circ} \mathrm{C}$ [9]. Thus it was expected to enhance the growth uniformity since it would completely decompose at the growth temperature range. However, InSb growth was unsuccessful in the $\mathrm{V} / \mathrm{III}$ ratio range of $10-230$. At V/III rations higher than 57, there was no film deposition for a growth during up to 20 minutes. At lower V/III ratios (higher In flow rates), only In droplets formed on the surface and white deposit was observed at the inlets of the reactor tube. These results were attributed to instability of TEIn, especially in the presence of hydrogen [9]. Therefore TMIn was used during the rest of the growth investigation on Sb-based system.

For the InSb growth using TMIn, the surface morphology was found to be very sensitive to the V/III ratio and closely reflected the crystallinity of the as-grown films. Based upon the morphology, only a narrow range of $\mathrm{V} / \mathrm{III}$ ratio around 11.5 at $465{ }^{\circ} \mathrm{C}$ was found to be optimum. At a lower V/III ratio around 9 the morphology degraded and the surface was covered with indium droplets. With an increase in V/III ratio the droplets were replaced

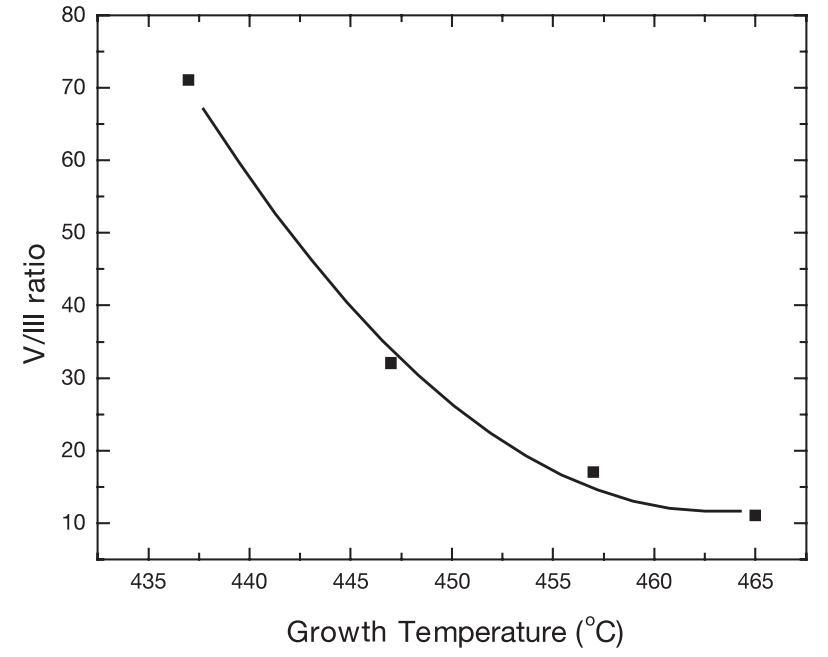

Fig. 3.2. Variation of the optimum V/III ratio as a function of deposition temperature for InSb grown using TMIn and TMSb.

Table 3.3. Optimum growth conditions for InSb.

\begin{tabular}{c|c}
\hline Growth temperature & $465{ }^{\circ} \mathrm{C}$ \\
\hline Growth pressure & 76 torr \\
\hline TMIn flow rate & $50 \mathrm{sccm}$ \\
TMSb flow rate & $20 \mathrm{sccm}$ \\
\hline Total $\mathrm{H}_{2}$ flow rate & $1.5 \mathrm{l} / \mathrm{min}$ \\
\hline
\end{tabular}

by hillocks whose density gradually decreased as the approach the optimum value. At higher V/III ratio than the optimum, the morphology deteriorates with Sb-related hillocks. The observed surface dependence on V/III ratio is related to the low vapor pressure of In and Sb. Therefore, excess In can easily deposit on the surface, leading vapor-liquid-solid (VLS) three-phase growth [10,11]. On the other hand, excess Sb forms a solid second phase which leads to the growth of hillocks; since $\mathrm{Sb}$ has a melting point of $630{ }^{\circ} \mathrm{C}$, VLS growth does not occur.

Similar studies of the V/III ratio dependence on the morphology have also been carried out at various temperatures. The optimum V/IIII ratio at each temperature is shown in Figure 3.2. It's noted that the optimum $\mathrm{V} / \mathrm{III}$ ratio increase with growth temperature decrease. This is due to incomplete decomposition of TMSb. While TMIn completely decomposes around $425^{\circ} \mathrm{C}[12]$, TMSb only starts decomposing at $400{ }^{\circ} \mathrm{C}$ and decomposition efficiency increases as the temperature increases [13]. Therefore, higher flow rate for TMSb is required at lower temperature. However growth carried out at temperature close to the melting point should be avoided since poor quality films are obtained from thermal degradation. Based on the results above, the optimum growth parameters have been determined and summarized in Table 3.3.

Another factor that was critical to morphology is the preparation of substrates. Substrates preparation only applied to InSb substrates since Superclean GaAs and GaAs-coated Si substrates did not require any pre-growth substrate preparation. A degreasing-etching method was 


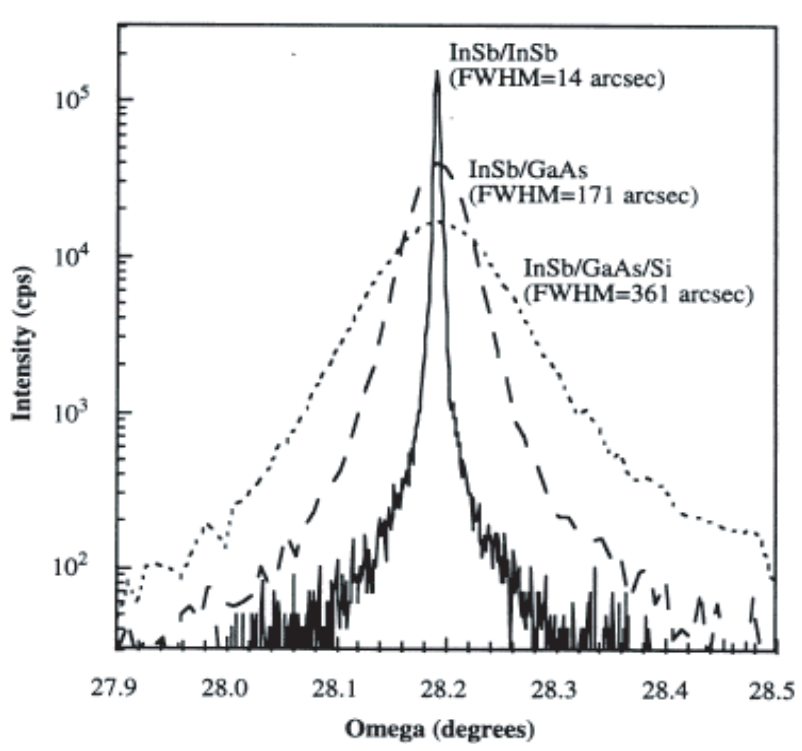

Fig. 3.3. X-ray rocking curve of $3 \mu \mathrm{m}$-thick InSb films grown on InSb, GaAs and GaAs-coated Si substrates.

chosen for the substrate preparation. Degreasing involved baths in boiling trichloroethylene, acetone, and proponol. Etching was carried out in a lactic acid: nitric acid (10:1) mixture for 7 minutes.

Under the optimum conditions, mirror-like InSb epilayers were grown on InSb, GaAs and Si substrates [14]. The comparison of X-ray FWHM of $3 \mu$ m-thick InSb layer was shown in Figure 3.3. The film grown on $\mathrm{InSb}$ showed X-ray FWHM of 14 arcs while those on GaAs and GaAs/Si had FWHM of 171 and 361 arcs, respectively. These broader peaks on GaAs and Si substrates are due to higher dislocation density from lattice mismatch.

For films grown on lattice mismatched substrates, the overall crystalline quality of the film is expected to depend on its thickness. Hence, X-ray diffraction measurements were performed on InSb films of various thicknesses on GaAs and Si substrates and the results are shown in Figure 3.4. The FWHM of InSb on both substrates decreases with the thickness increased, indicating improved crystalline quality way from the highly mismatched epilayersubstrate interface.

\subsubsection{Electrical characteristics}

Figure 3.5 shows the room temperature Hall mobility for InSb grown on GaAs substrates. The Hall mobility is found to improve with increasing thickness. Similar to the $\mathrm{X}$-ray results, this trend reflects the decrease of dislocation density away from the interface. The Hall coefficient RH was negative and the carrier concentration of the InSb was generally in the range of $1 \times 10^{16} \mathrm{~cm}^{-3}$ to $3 \times 10^{16} \mathrm{~cm}^{-3}$ at $300 \mathrm{~K}$, in good agreement with the intrinsic value determined from equation (3.2).

The Hall coefficient remained negative at $77 \mathrm{~K}$ but the Hall mobility decreased dramatically far below expected values for $n$-type InSb. This might indicate that the as-

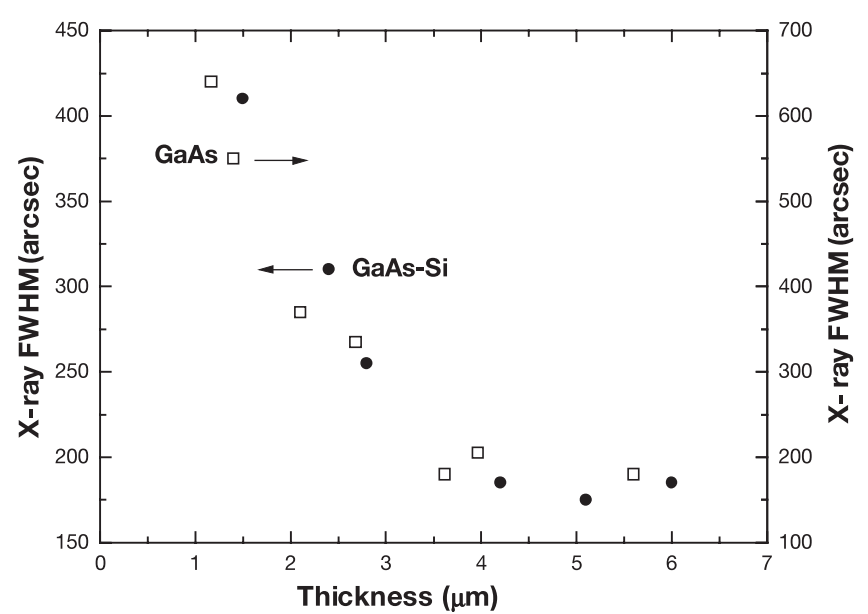

Fig. 3.4. Dependence of X-ray FWHM of heteroepitaxial InSb layers on film thickness.

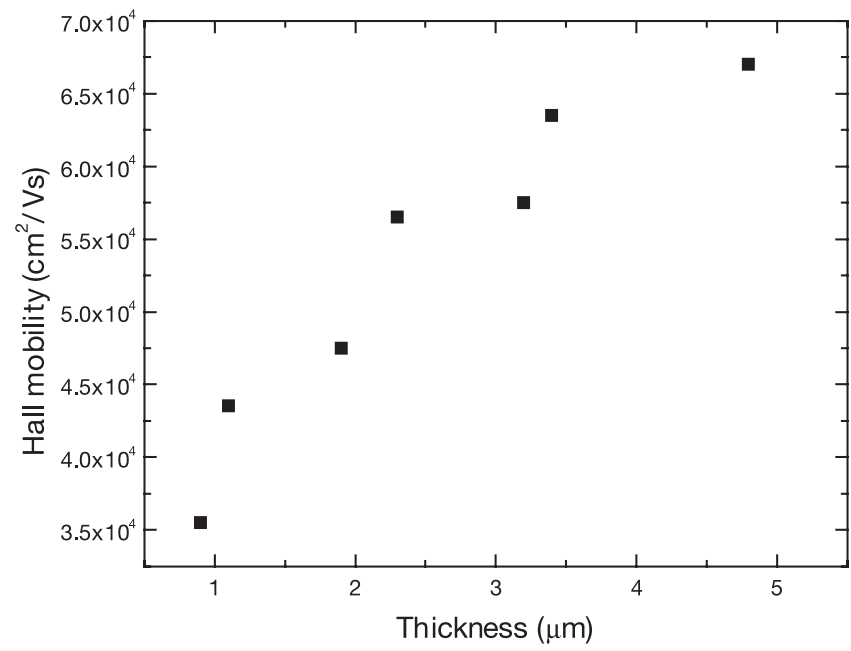

Fig. 3.5. Variation of room temperature Hall mobility of heteroepitaxial InSb layers on GaAs as a function of thickness.

grown layer is $p$-type. In order to verify this, more detailed analysis on this behavior has been done using a three-layer model [15]. Consisting of a surface electron accumulation layer as postulated by Soderstrom et al. [16], an interface layer with high density of dislocations [17], and a bulk like layer with a highly reduced defect density. The theoretical results with the assumption of $p$-type InSb layer showed good agreement with the experimental result verifying the growth of $p$-type InSb on GaAs substrates.

A special-grade TMSb was used instead of the electronic-grade TMSb in an attempt to improve the electrical properties. InSb films with thickness ranging from $1.2 \mu \mathrm{m}$ to $3.6 \mu \mathrm{m}$ were grown under the identical growth condition as previous growth. Compared with the previous sample grown with electronic-grade TMSb, the structural quality from X-ray diffraction was comparable and no noticeable change in the room temperature Hall mobilities were observed. However, the most striking difference were the low temperature electrical characteristics, as shown in Figure 3.6. The Hall mobility of the new InSb 


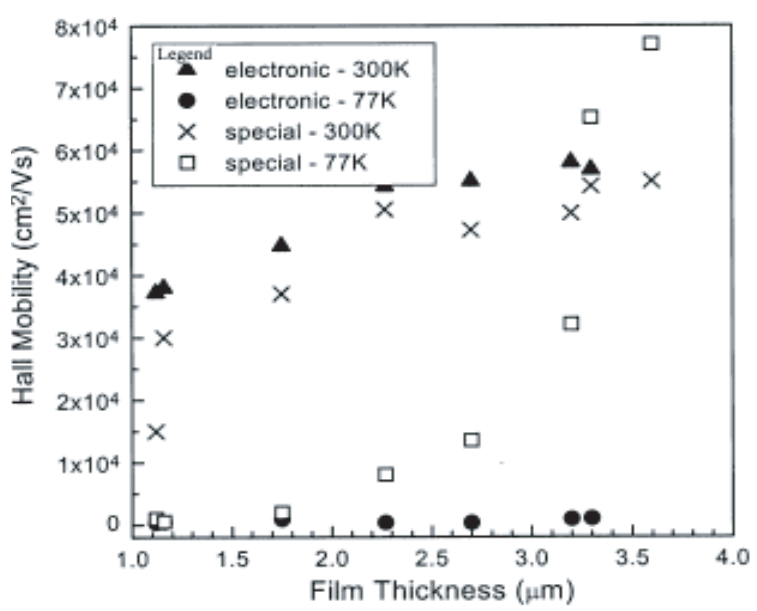

Fig. 3.6. Comparison of $300 \mathrm{~K}$ and $77 \mathrm{~K}$ Hall mobility of undoped InSb grown using electronic-grade and special-grade TMSb.

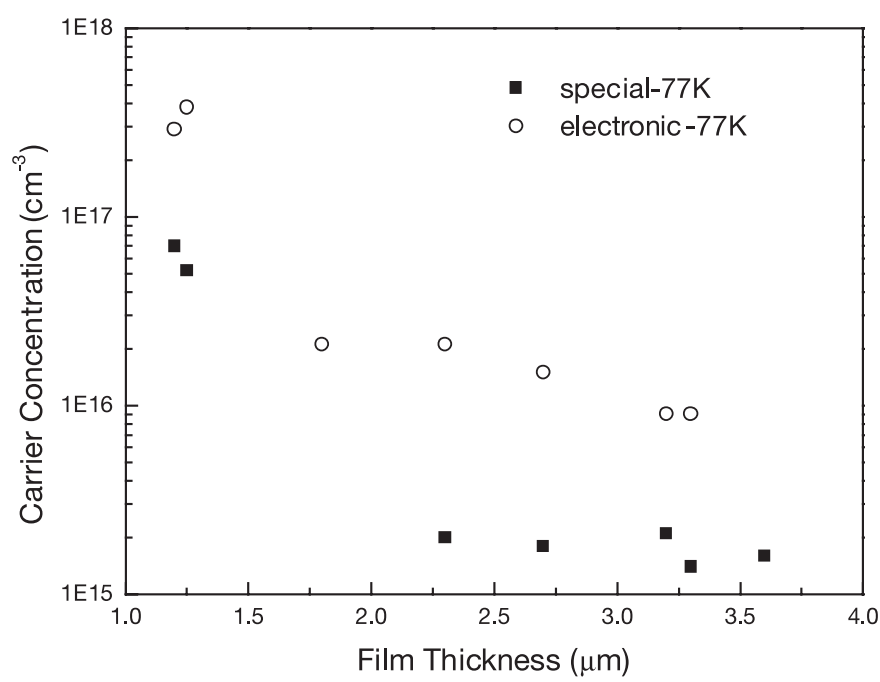

Fig. 3.7. Comparison of the $77 \mathrm{~K}$ carrier concentration of nominally undoped InSb grown with electronic-grade and specialgrade TMSb.

films remained relatively low for film thickness below $2 \mu \mathrm{m}$ while it drastically improved beyond this thickness. A $3.6 \mu \mathrm{m}$ thick InSb film showed mobility of $56000 \mathrm{~cm}^{2} / \mathrm{V} \mathrm{s}$ at $300 \mathrm{~K}$ which increased to about $80000 \mathrm{~cm}^{2} / \mathrm{V} \mathrm{s}$ at $77 \mathrm{~K}$. This temperature dependence of the Hall mobility differs from our previous result and resembles that of a $n$-type bulk material. The background carrier concentration at $77 \mathrm{~K}$ was comparable to the previous InSb samples up to $2 \mu \mathrm{m}$ but decreased by nearly an order of magnitude to $10^{15} \mathrm{~cm}^{-3}$ for thicker samples. These Hall data are comparable to the best epitaxially grown InSb results, irrespective of the growth technique.

Since the growth conditions are identical between the growths with electronic-grade and special grade-TMSb, the changes in electrical properties are attributed to an absence of some impurities in the special-grade TMSb that were otherwise present in the electronic-grade TMSb. The impurities, which have not yet been identified, most likely

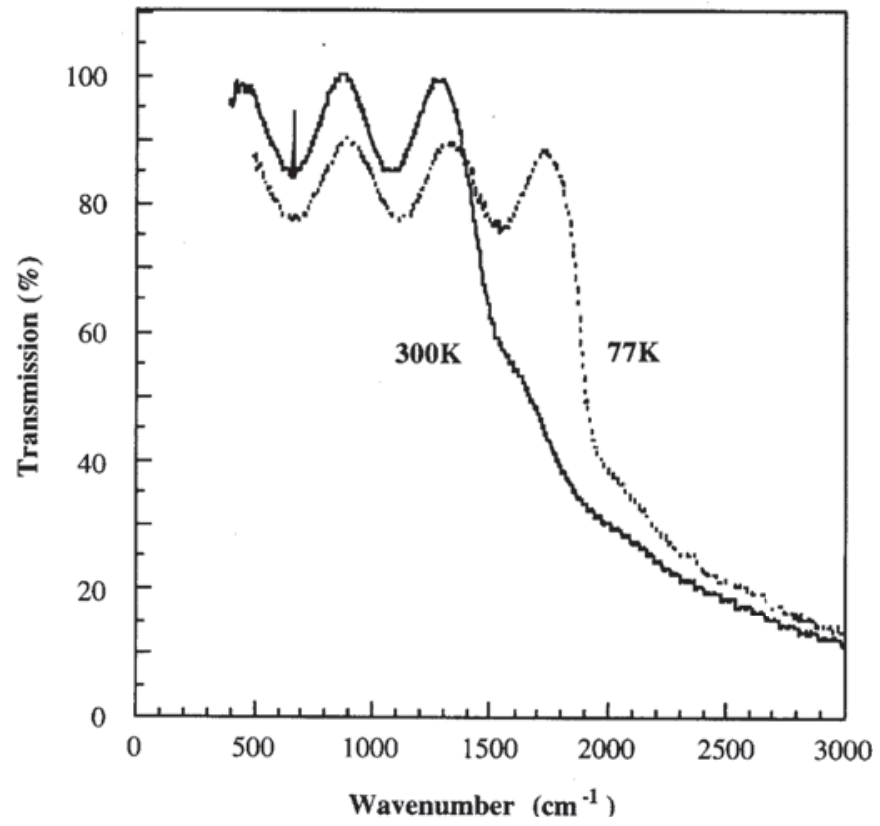

Fig. 3.8. Transmittance of spectra of $3.1 \mu$ m-thick InSb film on GaAs at $300 \mathrm{~K}$ and $77 \mathrm{~K}$.

provided a strong $p$-type background in the previous samples. In the new samples the background is predicted to be $n$-type since the Hall measurements reflected the highmobility electron characteristics.

\subsubsection{Optical characteristics}

Figure 3.8 shows the experimental transmittance spectra of $3.1 \mu \mathrm{m}$-thick InSb at $300 \mathrm{~K}$ and $77 \mathrm{~K}$. The spectra display a sharp absorption edge around $1200 \mathrm{~cm}^{-1}$ at $300 \mathrm{~K}$ which shifts to $1700 \mathrm{~cm}^{-1}$ at $77 \mathrm{~K}$; however, an accurate assessment of the absorption edge is difficult because of Fabry-Perot oscillations. The period of the oscillations yields the phase shift for light traveling trough the epilayer, from which the product $n \cdot d$ can be extracted where $n$ is the refractive index of the epilayer, and $d$ is the thickness.

From the transmission measurements, the absorption coefficient of InSb was extracted. To take Fabry-Perot oscillation into account we have modeled the InSb epilayer as a conventional Fabry-Perot cavity filled with absorbing medium. The reflection and transmission coefficient of each interface were determined using tabulated values for the refractive index of GaAs substrate and InSb. The phase shift through the epilayer was determined from the position of the experimental extrema. The extracted absorption spectrum is shown in Figure 3.9.

In order to accurately determine the bandgap, photoconductivity measurements were carried out. Due to the high thermal background noise at room temperature, the measurement has been performed at $77 \mathrm{~K}$. Figure 3.10 shows the spectral responses of the $3.1 \mu \mathrm{m}$-thick InSb film grown using electronic-grade TMSb (lower response) and an InSb film of comparable thickness grown using special 


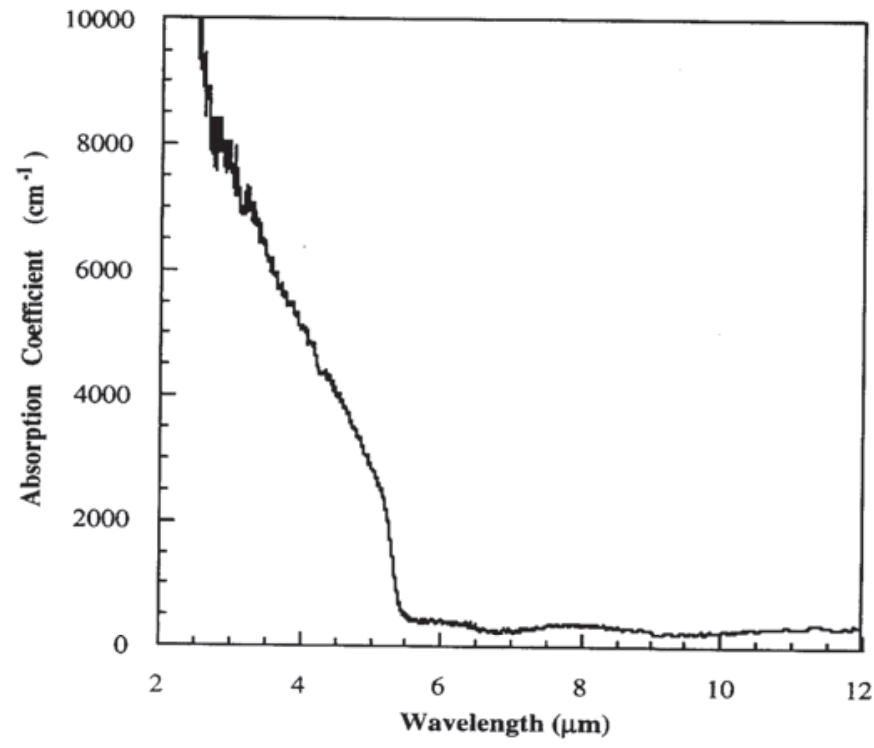

Fig. 3.9. Extracted absorption spectrum of $3.1 \mu \mathrm{m}$-thick InSb film on GaAs at $77 \mathrm{~K}$.

TMSb (higher response). Both responses were measured under the same conditions and they show a sharp cutoff at the $5.5 \mu \mathrm{m}$ which corresponds to a bandgap energy of $0.23 \mathrm{eV}$. The narrow feature around $4.2 \mu \mathrm{m}$ is due to the absorption by carbon dioxide in the surrounding environment. The large difference in the absolute responsivity is attributed to the photoconductive gain $G$ of the samples since the quantum efficiency, which depends on the thickness, is comparable for the two samples. Therefore, a higher gain, which is related to the mobility-lifetime product following the simple photoconductivity theory [18], reflects the improved mobility and the lower background carrier concentration of the samples grown using specialgrade $\mathrm{TMSb}$.

\subsubsection{Doping characteristics}

\subsubsection{4.a N-type}

TESn was used as $n$-type dopant. The TESn bubbler was kept at a temperature of $-25{ }^{\circ} \mathrm{C}$ and the flow rate was varied from $7 \mathrm{sccm}(0.7 \mathrm{nmol} / \mathrm{min})$ to $80 \mathrm{sccm}$ $(8.0 \mathrm{nmol} / \mathrm{min})$, resulting doping level from $5 \times 10^{16} \mathrm{~cm}^{-3}$ to $1.2 \times 10^{18} \mathrm{~cm}^{-3}$, as shown in Figure 3.11. No degradation in morphology and X-ray FWHM were observed. Furthermore, there was no noticeable memory effect associated with TESn.

\subsubsection{4.b P-type}

$P$-doping was first carried out using DMZn. There was no observable effect on the growth rate or the morphology. With a 10 sccm capacity mass flow controller, a controllable doping level no lower than $3 \times 10^{18} \mathrm{~cm}^{-3}$ was obtained. The doping level remained steady down to $4 \mathrm{~K}$

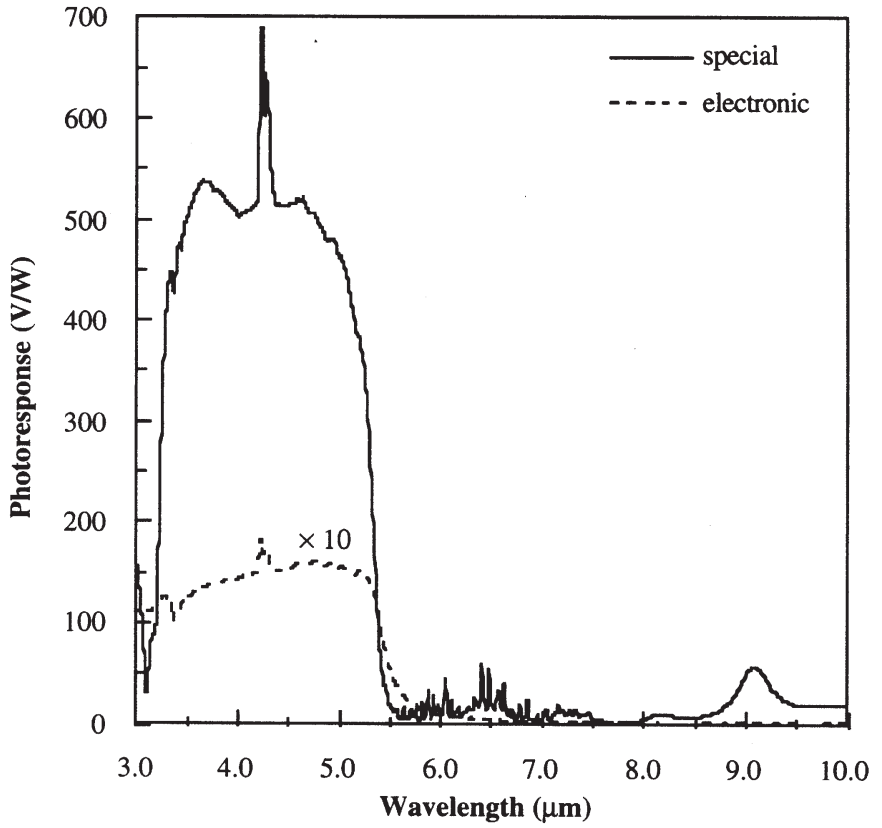

Fig. 3.10. Comparison of the $77 \mathrm{~K}$ photoresponse of $\mathrm{InSb} / \mathrm{GaAs}$ grown using special-grade and electronic-grade TMSb. The thickness of the two samples was comparable $(3.1 \mu \mathrm{m})$. The photoresponse of the "electronic-grade" sample has been multiplied by a factor of 10 .

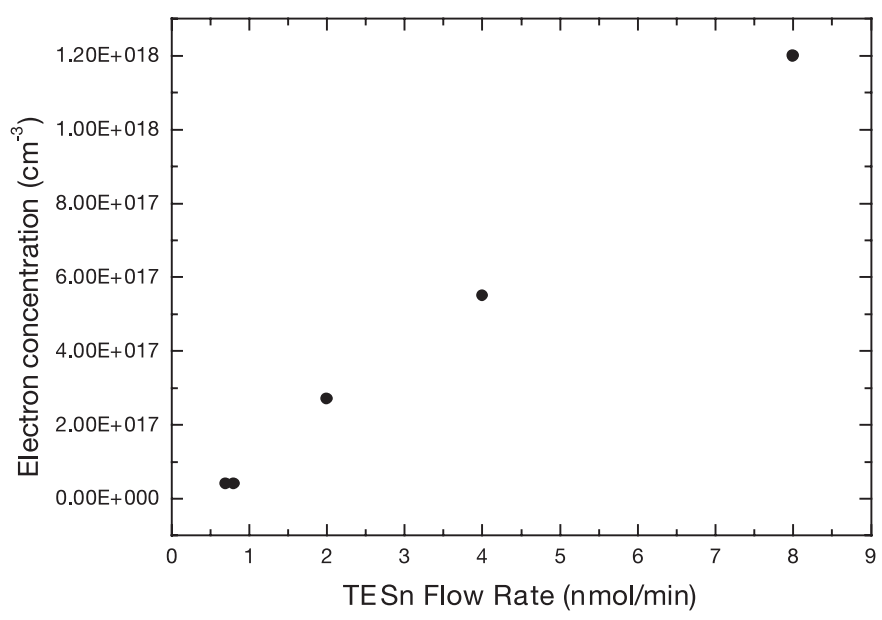

Fig. 3.11. Epitaxial Sn-doping of InSb.

without any singularity, which indicates complete ionization of the acceptors as well as absence of noticeable surface and interface layer contributions.

In order to expand the $p$-doping levels to lower ranges, DEZn was considered because of its lower vapor pressure. With the bubbler temperature at $10{ }^{\circ} \mathrm{C}$, the flow of DEZn was varied from $0.05 \mathrm{sccm}(0.02 \mu \mathrm{mol} / \mathrm{min})$ to $0.2 \mathrm{sccm}$ $(0.08 \mu \mathrm{mol} / \mathrm{min})$. As shown in Figure 3.12 these flows yield doping levels from $2.5 \times 10^{17} \mathrm{~cm}^{-3}$ to $7.5 \times 10^{17} \mathrm{~cm}^{-3}$. In conjunction with the results of DMZn, the successful Zn-doping of InSb in the range of $10^{17}-10^{19} \mathrm{~cm}^{-3}$ was achieved, which is sufficient for the $p^{+}$-layer in InSb photodiode structure. 


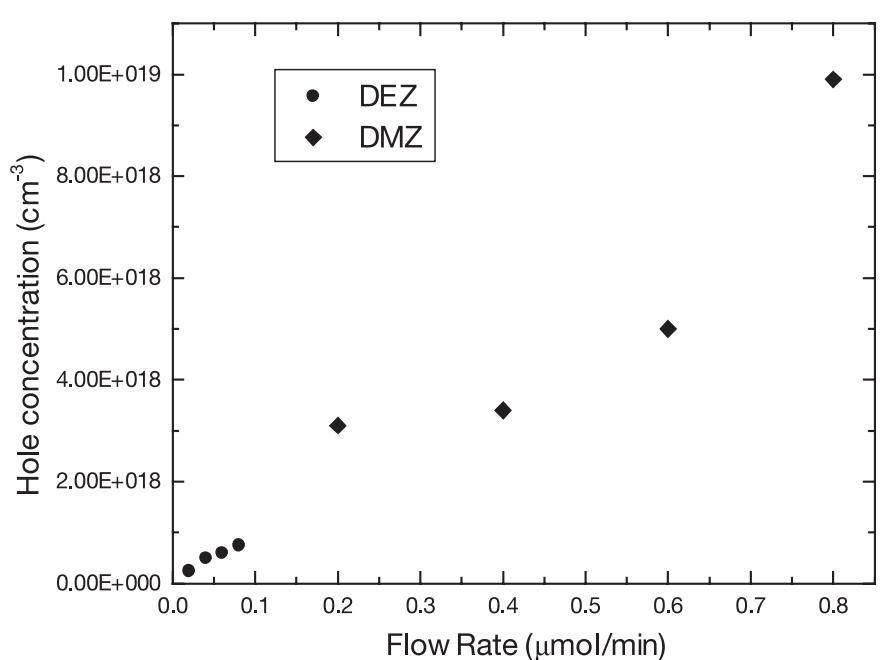

Fig. 3.12. Epitaxial Zn-doping of InSb.

\subsection{MBE growth of InSb}

\subsubsection{InSb on (100) GaAs}

The growth of InSb was optimized using an EPI Modular Gen II Solid source MBE system using elemental uncracked source. Group III materials were $6 N$ (six nines$99.9999 \%$ ) pure and the group $\mathrm{V}$ were $7 N$ pure. The system is equipped with a RHEED system for calibrating growth rate and monitoring the growth surface as well as a mass spectrometer for monitoring molecular species in the chamber. Substrates were epi-ready GaAs (or GaAs/Si).

Growth conditions were calibrated using RHEED oscillations for careful control of the V/III ratio, and static RHEED for monitoring the surface temperature. Optimum conditions on (100) GaAs were found to be 1.2/1.0 $\mathrm{Sb} / \mathrm{In}$ incorporation rate and a substrate temperature $5{ }^{\circ} \mathrm{C}$ above the transition temperature $\left(\sim 395^{\circ} \mathrm{C}\right)$. This gave excellent material quality with a mirror-like surface across the entire wafer.

\subsubsection{Structure characteristics}

The structural quality was examined with 5-crystal $\mathrm{X}$-ray diffraction. Excellent uniformity is indicated by the \pm 3 arcs variation of the rocking curve FWHM for a $10 \mu \mathrm{m}$ InSb layer on a 3" semi-insulating GaAs substrate. The X-ray FWHM decreases with thickness as the dislocation density decreases due to the greater distance between the surface and the highly dislocated interface as shown in Figure 3.13.

\subsubsection{Electrical characteristics}

Electrical characterization of InSb on GaAs was obtained using Hall measurements. As with the X-ray, the electron mobility increased with thickness. Electron mobility versus epilayer thickness is shown in Figure 3.14.

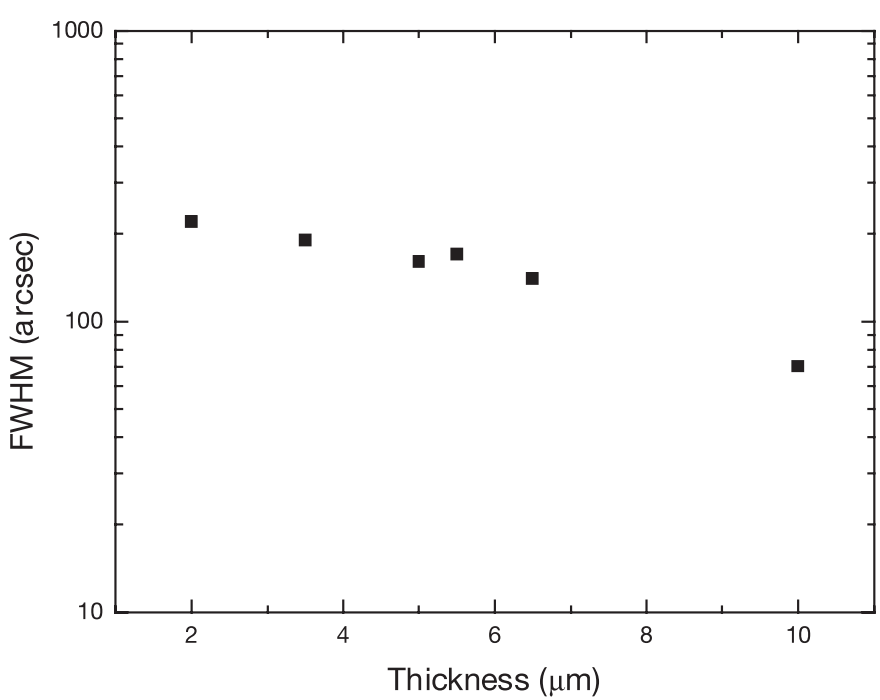

Fig. 3.13. X-ray FWHM versus epilayer thickness.

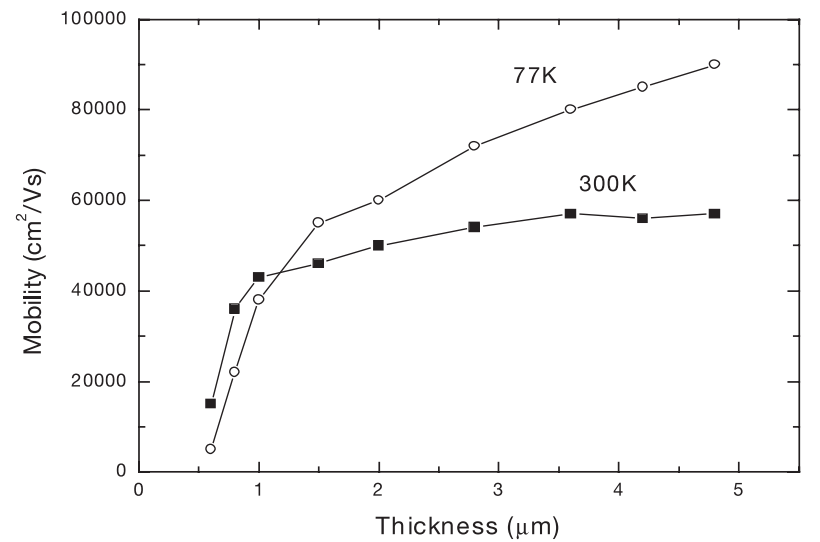

Fig. 3.14. Electron mobility versus $\mathrm{InSb}$ epilayer thicknesses on (100) GaAs at $77 \mathrm{~K}$ and $300 \mathrm{~K}$.

The $300 \mathrm{~K}$ mobility levels are near that of bulk InSb $\left(75000 \mathrm{~cm}^{2} / \mathrm{V} \mathrm{s}\right)$ as the thickness is more than $2 \mu \mathrm{m}$, while the $77 \mathrm{~K}$ mobility continues to increase. The data in Figure 3.15 is from material grown with $6 N$ source, which resulted in a background concentration of $10^{16} \mathrm{~cm}^{-3}$. Mobility measurement on the $6 N$ purity material showed typical $n$-type characteristics as shown in Figure 3.15. The peak in mobility at $77 \mathrm{~K}$ is typical of $n$-type material, and the sharp drop below $77 \mathrm{~K}$ is most likely caused by the dislocation scattering at the InSb/GaAs interface.

Subsequent cell charge with $7 N$ resulted in a background concentration of $10^{15} \mathrm{~cm}^{-3}$. All materials grown with this higher purity source showed slightly higher mobility at $300 \mathrm{~K}$, but drastically decreased mobility $\left(<5000 \mathrm{~cm}^{2} / \mathrm{Vs}\right)$ at $77 \mathrm{~K}$. Similar to the discussion of in Section 3.2.1.2, the three layer model for MOCVD material was applied here to explain why the $77 \mathrm{~K}$ mobility could be so low while excellent material quality was indicated by RHEED, X-ray, room temperature mobility and the device characteristics. In this analysis, the epilayer is assumed to be made of 3 layers: an interface layer with high dislocation density, a bulk-like layer, and a 


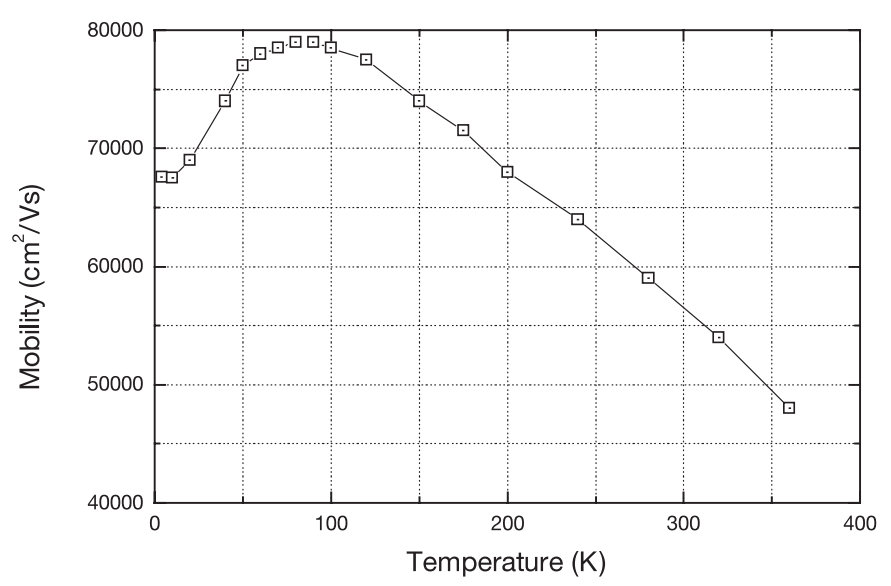

Fig. 3.15. Electron mobility versus temperature for a $3.3 \mu \mathrm{m}$ InSb on (100) GaAs.

surface inversion layer. When the lower purity Sb source was used, the higher background concentration ensured that the $77 \mathrm{~K}$ mobility measurements characterized the high mobility bulk-like layer as well as the interface and surface layers. However, with the higher purity Sb source, the bulk-like layer is ten times less conductive due to the order of the magnitude decrease in carrier concentration, which makes the high dislocation density interface layer to dominate. When the material was doped $n$-type in the range of $5 \times 10^{15} \mathrm{~cm}^{-3}-1 \times 10^{16} \mathrm{~cm}^{-3}$, the $77 \mathrm{~K}$ mobility would be leveled up to that of lower purity Sb result. This, however, did not rule out the possibility of compensation, as the $n$-type doping would negate the inversion and would again make the bulk-like layer more significant.

However, the possibility of compensation was effectively eliminated by the following experiment. Several thick InSb/GaAs sample grown with the higher purity $\mathrm{Sb}$ were epoxied to glass slides and the substrates were etched off leaving only the InSb epilayer with mirrorlike morphology. A $5 \mu \mathrm{m}$ sample exhibited mobility of $65000 \mathrm{~cm}^{2} / \mathrm{Vs}$ at $300 \mathrm{~K}$, and $95000 \mathrm{~cm}^{2} / \mathrm{Vs}$ at $77 \mathrm{~K}$. Before removing the substrates, this sample had mobility of $60000 \mathrm{~cm}^{2} / \mathrm{Vs}$ at $300 \mathrm{~K}$ and $4230 \mathrm{~cm}^{2} / \mathrm{Vs}$ at $77 \mathrm{~K}$. This indicated that the InSb/GaAs interface was indeed dominating the $77 \mathrm{~K}$ measurement. This etching procedure also shows that the interface does not preclude the growth of highly quality InSb away from the interface.

\subsubsection{Optical characteristics}

The optical quality of the material was determined using photoluminescence (PL), transmission, and photoresponse measurement. The PL linewidth of InSb/GaAs was $18 \mathrm{meV}$ at $77 \mathrm{~K}$. Figure 3.16 shows the PL and photoresponse measurements of a $4.8 \mu \mathrm{m}$ InSb on GaAs sample.

Transmission measurements were performed using a Mattson Fourier Transform Infrared (FTIR) spectrometer. Figure 3.17 shows the transmission spectrum for $9.2 \mu \mathrm{m}$ sample. The Fabry-Perot oscillations were used to

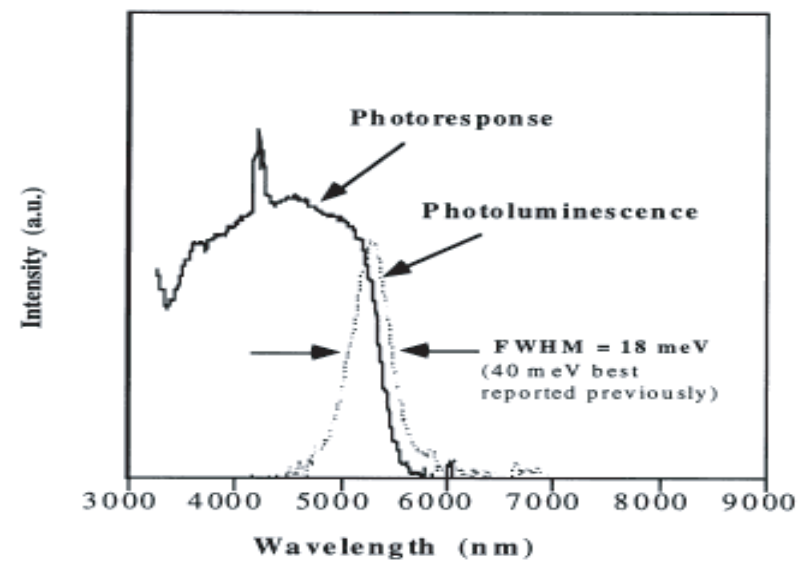

Fig. 3.16. PL and photoresponse of a $4.8 \mu \mathrm{m} \mathrm{InSb}$ on GaAs sample.

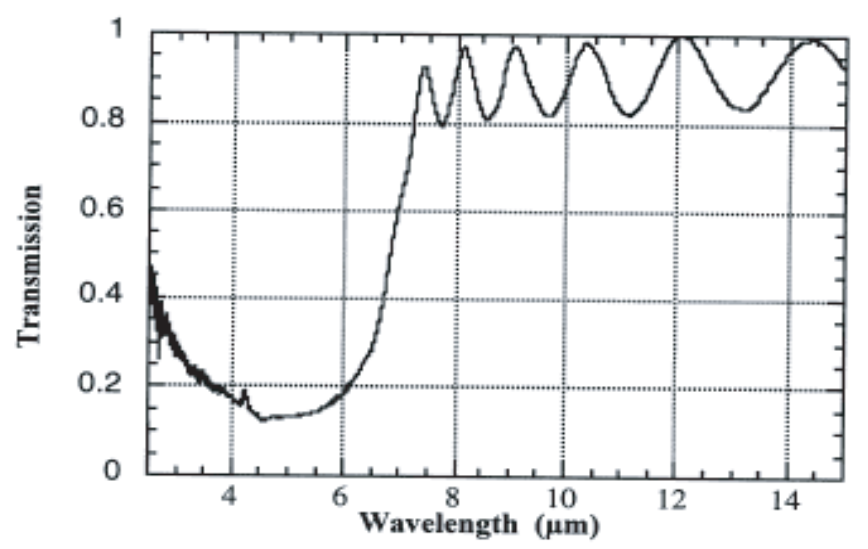

Fig. 3.17. Normalized transmission spectrum for a $9.2 \mu \mathrm{m}$ InSb epilayer on GaAs.

verify the epilayer thickness and were in excellent agreement with the thickness expected from RHEED oscillations and was verified by Scanning electron microscopy (SEM). The absorption coefficient estimated from the transmission spectra obtained on these InSb sample was $\sim 3400 \mathrm{~cm}^{-1}$ at $4 \mu \mathrm{m}$, which is smaller than that of bulk $\mathrm{InSb}$.

Time-solved PL measurements were made to examine carrier lifetime across 3" wafer. Carrier life time of $>200 \mathrm{~ns}$ at $77 \mathrm{~K}$ were obtained, which is comparable to that of bulk $\mathrm{InSb}(\sim 300 \mathrm{~ns})$.

\subsubsection{InSb on (111)B GaAs}

Growth of InSb on (111) GaAs was investigated [19] because properly passivated (111) surfaces have been found to have lower surface recombination and provide better electrical isolation than (100) surfaces [20]. The substrates used for this study were 3" (111) GaAs from Lockheed Martin Fairchild Systems.

The growth conditions which were varied to determine optimum conditions for InSb growth on (111) GaAs were the $\mathrm{Sb} / \mathrm{In}$ ratio and the substrate temperature. The 


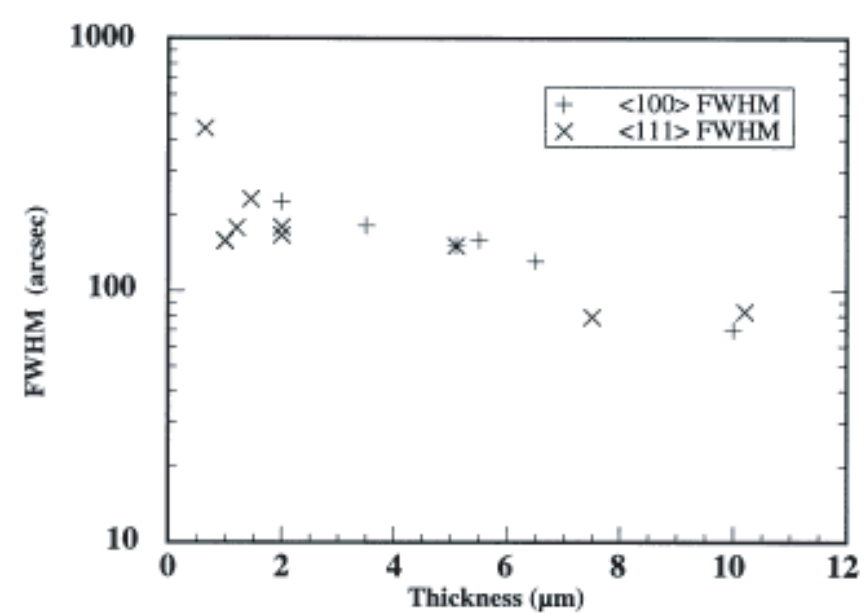

Fig. 3.18. X-ray FWHM versus epilayer thickness for InSb on (100) and (111) GaAs.

$\mathrm{Sb} / \mathrm{In}$ ratio was varied from 1.0 to 1.5 , and the substrate temperature was varied from 380 to $460{ }^{\circ} \mathrm{C}$. The optimum conditions were found to be different for the growth of InSb on (111) B surfaces compared to those for (100) surfaces as described previously. For a given $\mathrm{Sb} / \mathrm{In}$ ratio, it was found that the optimum growth temperature was higher for the growth of InSb on (111) B surfaces than on (100) surfaces. This difference has been attributed to the bonding characteristics at the surface. The (111) B surface has three dangling bonds for $\mathrm{Sb}$ atoms and one dangling bond for In atoms, whereas the (100) surfaces has two bonds for both atoms.

The epilayers were first characterized using high resolution X-ray diffraction. The X-ray FWHM versus thickness measurements in Figure 3.18 shows that the crystallinity is nearly identical for (100) and (111) B InSb at a given thickness under optimum growth conditions. As expected, the crystallinity improves with increasing thickness, but there is no large increase in the FWHM for thinner samples, which might indicate that the crystallinity is optimized under theses growth conditions.

The substrate temperature has a greater effect than thickness on the crystallinity as shown in Figure 3.19. The optimum crystallinity is obtained with growth temperature between 420 and $440{ }^{\circ} \mathrm{C}$, whereas, for (100), the optimum crystallinity is obtained for samples grown at $\sim 395{ }^{\circ} \mathrm{C}$. Again, the difference in optimum growth temperature is most likely due to the difference of dangling bonds at the surfaces.

Figure 3.20 shows the room temperature electron mobility as a function of epilayer thickness for InSb grown on (100) and (111) B GaAs. It can bee seen that the data of (111) B and (100) GaAs agrees with each other exceptionally well. It appears that when InSb is grown under optimized condition on (111) B and (100) GaAs, the quality of the material produced is independent of the substrate orientation.

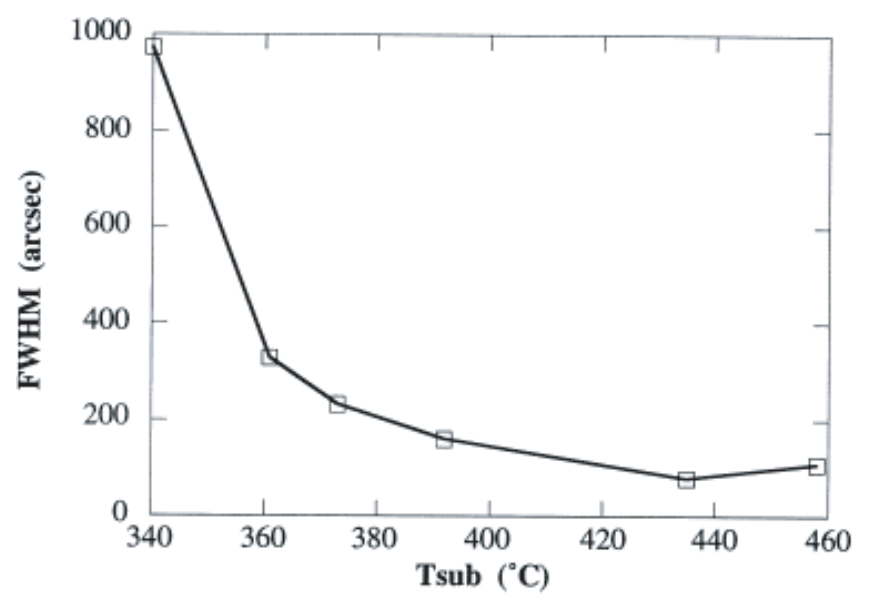

Fig. 3.19. X-ray FWHM versus growth temperature.

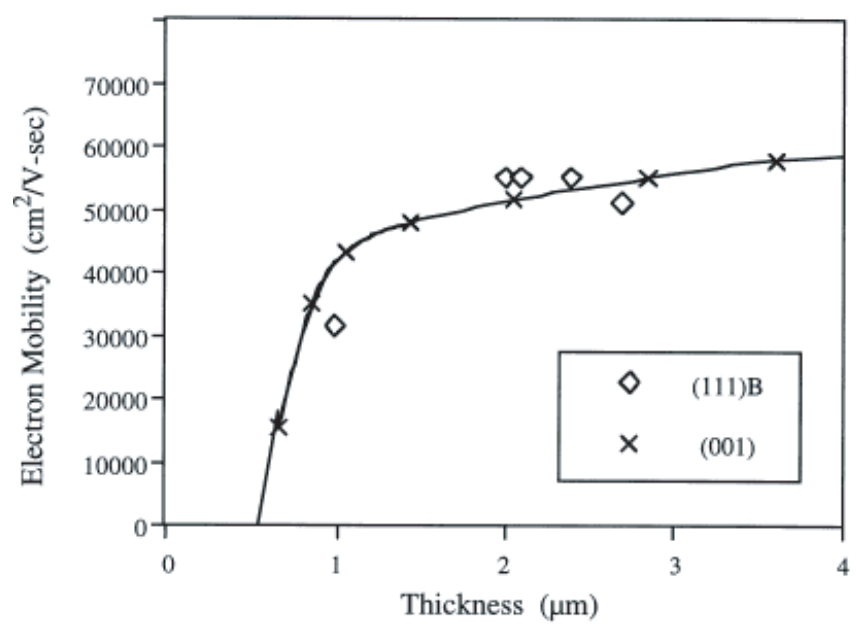

Fig. 3.20. Electron mobility versus epilayer thickness for InSb on (100) and (111) GaAs.

\subsubsection{InSb photodetectors}

\subsubsection{InSb photodiodes}

The InSb photodiodes were grown on 3" $\mathrm{Si}$ and (111) GaAs substrates. The InSb photodiodes typically consisted of a $2 \mu \mathrm{m} n^{+}$region $\left(\sim 10^{18} \mathrm{~cm}^{-3}\right.$ at $\left.77 \mathrm{~K}\right)$, a $\sim 6 \mu \mathrm{m}$ unintentionally doped region $\left(n \sim 10^{15} \mathrm{~cm}^{-3}\right.$ at $77 \mathrm{~K})$ and $\mathrm{a} \sim 0.5 \mu \mathrm{m} p^{+}\left(\sim 10^{18} \mathrm{~cm}^{-3}\right)$ contact layer. The crystallinity of these structure was excellent as confirmed by the X-ray diffraction, which showed FWHM $<100$ arcs for structures grown on (100) and (111) GaAs and Si substrates. Photodiodes were fabricated with $400 \times 400 \mu \mathrm{m}^{2}$ mesa structures by photolithography and wet chemical etching. Au/Ti Ohmic contacts for both $n$ - and $p$-type layers were made by an e-beam evaporator. The contact pattern was defined again by standard photolithograpy and selective etching (KI: $\mathrm{I}_{2}: \mathrm{H}_{2} \mathrm{O}$ for $\mathrm{Au}$ and $\mathrm{HF}$ for $\mathrm{Ti}$ etching). The chips were assembled using standard circuit technologies including die separation, mounting and $\mathrm{Au}-$ wire bonding.

The relative photoresponse of these devices was measured using FTIR system. The absolute responsivity was 


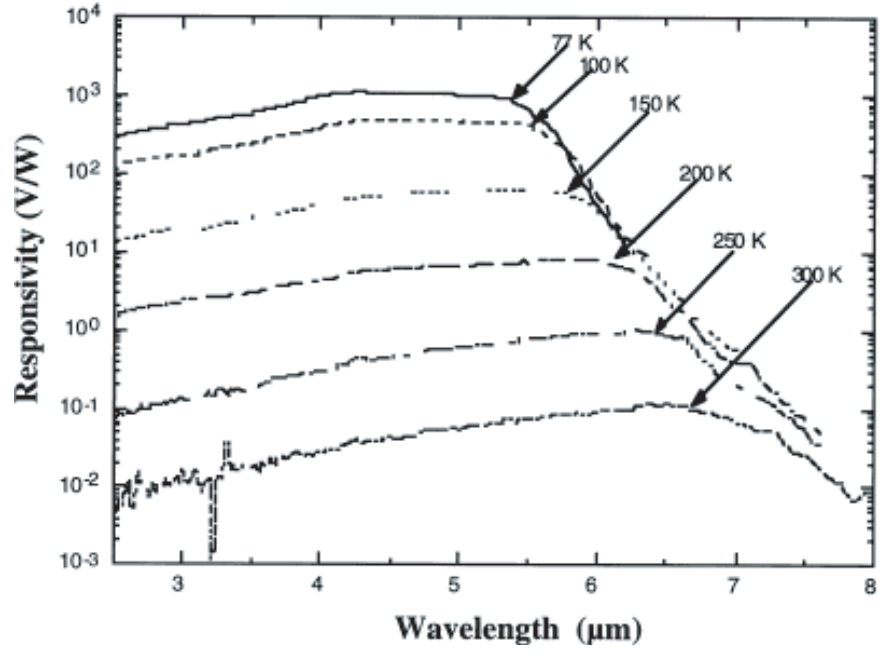

Fig. 3.21. Absolute photoresponse for an InSb $p-i-n$ photodetector on Si.

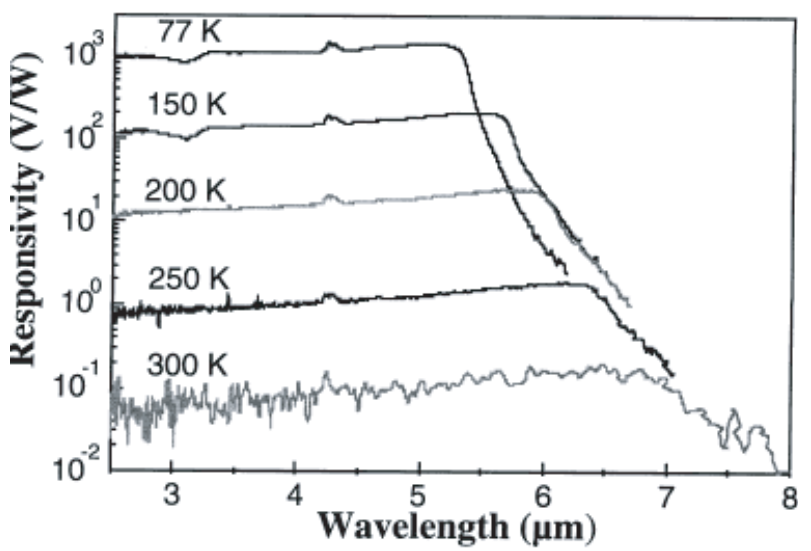

Fig. 3.22. Absolute photoresponse for an InSb $p-i-n$ photodetector on (111) B GaAs.

obtained by measuring the signal using a calibrated blackbody source and a lock-in amplifier system. The absolute responsivity for $p-i-n$ detectors on $\mathrm{Si}$ and (111) GaAs are shown in Figures 3.21 and 3.22.

These devices showed excellent response of about $1000 \mathrm{~V} / \mathrm{W}$, which is comparable to that of bulk detectors, with detectivities of $\sim 3 \times 10^{10} \mathrm{~cm} \mathrm{~Hz} / \mathrm{W}$ at $77 \mathrm{~K}$. Note that the detectors operate up to room temperature, even though these devices were not optimized for room temperature operation.

\subsubsection{InSb focal plane array}

In collaboration with Lockheed Martin Fairchild systems, $256 \times 256$ InSb FPA's were fabricated from InSb layers on 3" GaAs and Si substrates. Starting from an undoped $10 \mu \mathrm{m}$ InSb epilayer, the $p$-type regions were ion implanted. The structures were then chemically etched to form mesas, and In-bump-bonded to Si CMOS readout circuitry. Good imaging was obtained as shown in Figure 3.23 .
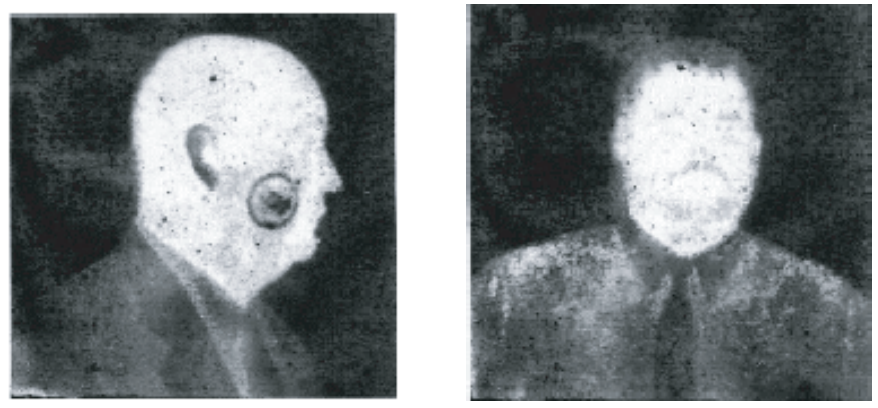

Fig. 3.23. IR images obtained from an InSb on GaAs FPA.

\section{$4 \ln A s S b$}

\subsection{Introduction}

The cutoff of InSb is $\sim 5.5 \mu \mathrm{m}$ at $77 \mathrm{~K}$, and is $\sim 7 \mu \mathrm{m}$ at $300 \mathrm{~K}$. Many applications require longer wavelength detection. Since $300 \mathrm{~K}$ radiation has a peak around 9-11 $\mu \mathrm{m}$, IR detectors which detect in this region are useful for thermal imaging applications. Thus, there is a great amount of work in IR detector material covering $8-12 \mu \mathrm{m}$ wavelength region.

Among III-V semiconductor alloys, InAsSb has the lowest bandgap (with the exception of Tl- and Bicontaining alloys). The experimental results showed that InAsSb can cover the entire 8-14 $\mu \mathrm{m}$ range at near ambient temperature $(200 \mathrm{~K}-300 \mathrm{~K})$, it is a promising material system fore the uncooled photon detectors for the LWIR applications.

\subsubsection{Physical properties}

\subsubsection{Bandgap}

The $\operatorname{InAs}_{1-x} \mathrm{Sb}_{x}$ alloy has a zinc blende structure and direct gap at the Brillouin zone center. The shape of the electron band and the light hole band is determined by the $\mathbf{k p}$ theory.

According to Wieder and Clawson the energy gap of $\operatorname{InAs}_{1-x} \mathrm{Sb}_{x}$ may be described by the following expression [21]:

$$
\begin{aligned}
E_{g}(x, T)=0.411-\frac{3.4 \times 10^{-4} T^{2}}{210}+ & T \\
+ & 0.876 x+0.70 x^{2} \\
& \times 10^{-4} x T(1-x)
\end{aligned}
$$

Some recent studies show that the minimum band gap can be considerably less compared to that predicted by equation (4.1). Bethel et al. [22,64] estimated $300 \mathrm{~K}$ bad gap of $\mathrm{InAs}_{0.22} \mathrm{Sb}_{0.78}$ to be $0.095 \mathrm{eV}$. They also measured significant photoconductivity at $10 \mu \mathrm{m}$ at $77 \mathrm{~K}$. Chiang and Bedair [23]. reported $\sim 9 \mu \mathrm{m}$ cutoff wavelength of $x=0.6$ photovoltaic detector operating at $77 \mathrm{~K}$. Our $300 \mathrm{~K}$ absorption measurements give $\alpha=500 \mathrm{~cm}^{-1}$ for $\lambda=15 \mu \mathrm{m}$ for $x=0.65$ which corresponds to $E_{g}=0.083 \mathrm{eV}$. Therefore, the practical limitation for the long wavelength operation at room temperature is not due to wide gap but rather to high generation recombination rate. 


\subsubsection{Effective masses}

To obtain a good agreement between experimental room temperature effective masses and calculations, Rogalski and Jozwikowski [24] have taken into account the conduction-valence-band mixing theory. In this approximation, the resultant conduction-band effective mass can be written as:

$$
\begin{aligned}
& \frac{1}{m_{e}^{*}}=\frac{1}{m_{c e}^{*}}+\frac{\delta E}{3}\left[\frac{1}{m_{h h}^{*} E_{g v}}+\frac{1}{m_{l h}^{*} E_{g v}}\right. \\
& \left.\quad+\frac{1}{m_{s}^{*}\left(E_{g v}+\Delta_{v}\right)}-\frac{1}{m_{c e}^{*}}\left(\frac{2}{E_{g v}}+\frac{1}{\left(E_{g v}+\Delta_{v}\right)}\right)\right]
\end{aligned}
$$

where $\delta E=E_{g v}-E_{g} . E_{g v}$ and $\Delta_{v}$ can be approximated by expressions: $E_{g v}=0.351-0.176 x$ and $\Delta_{v}=0.39+0.42 x$. $m_{h h}^{*}, m_{l h}^{*}$, and $m_{s}^{*}$ are the effective masses of heavy-hole, light-hole, and split-off band, respectively. Finally, $m_{c e}^{*}$ is the effective mass for the conduction band in the absence of conduction band mixing. The $m_{e}^{*}(x)$ dependence obtained from expression (4.2) gives a good agreement with the experimental data.

\subsubsection{Intrinsic carrier concentration}

The intrinsic carrier concentration in $\operatorname{InAs}_{1-x} \mathrm{Sb}_{x}$ as a function of $x$ for various temperatures has been calculated in terms of the $\mathbf{k p}$ theory. In the calculation, the nonparabolic bands were taken into account and a parabolic shape of the heavy hole band was assumed. By fitting the calculated nonparabolic $n_{i}$ value to the expression for parabolic bands, the following approximation for the intrinsic carrier concentration has been obtained [24]

$$
\begin{array}{r}
n_{i}=\left(1.35+8.50 x+4.22 \times 10^{-3} T-1.53 \times 10^{-3} x T\right. \\
\left.-6.73 x^{2}\right) \times 10^{14} T^{3 / 2} E_{g}^{3 / 4} \exp \left(-E_{g} / 2 k T\right)
\end{array}
$$

where $n_{i}$ is in $\mathrm{cm}^{-3}$.

There is little information available on the physical properties of InAsSb alloys. It is reasonable to assume that the InAsSb ternary semiconductor will exhibit similar properties to both InAs and InSb, although additional process occurs, e.g. an additional scattering process due to the random scattering potential. Table 4.1 summarizes material parameters for InAs, $\operatorname{InAs}_{0.35} \mathrm{Sb}_{0.65}$, and InSb.

\subsection{MOCVD growth of inAsSb}

For the growth investigation of InAsSb a test structure, consisting of an InSb buffer layer grown for 30 minutes followed by an InAsSb layer for 2 hours, was adopted. $\mathrm{InSb}$ buffer layers were grown under the optimum conditions determined from InSb investigation (as described in Sect. 2). The growth of InAsSb layers has been performed in the following sequences. First, TMIn flow was directed to vent line to interrupt the growth of InSb. Then under the TMSb overflow, the flow rate of TMIn was decreased to about $90 \%$ of the original value for the InSb growth. It was found experimentally that V/III ratio needs to be increased for the InAsSb growth. This might be caused by the parasitic reaction between Arsine and TMSb. Third step, Arsine flow was introduced into the growth chamber. TMIn was redirected to growth chamber as the last step to initiate the growth of InAsSb. The average growth rate of these layers was about $0.8 \mu \mathrm{m} / \mathrm{h}$.

\subsubsection{Characterizations}

\subsubsection{Structural characteristics}

The grown layer surfaces are mirror-like. The alloy composition was estimated from X-ray diffraction using Vegard's law. At first, the dependence of Sb composition of InAs $\mathrm{As}_{1-x} \mathrm{Sb}_{x}$ films on various parameters has been studied. Keeping the TMSb flow at $35.4 \mu \mathrm{mol} / \mathrm{min}$ and $\mathrm{AsH}_{3}$ flow at $13.4 \mu \mathrm{mol} / \mathrm{min}$, flow of TMIn has been varied from 3.06 to $4.60 \mu \mathrm{mol} / \mathrm{min}$ at a growth temperature of $465{ }^{\circ} \mathrm{C}$. The corresponding V/III ratio varied from 15.93 to 10.62 . Further increase in the $\mathrm{V} / \mathrm{III}$ ratio resulted in the lower crystal quality and decrease in the V/III ratio caused In related droplet on the surface. As V/III ratio was increased, the composition of $\mathrm{Sb}$ was decreased from 0.8 to 0.63 (as shown in Fig. 4.1). The morphology and crystallinity dependence of the InAsSb layers on the $\mathrm{V} / \mathrm{III}$ ratio was not as critical as that of InSb layer. However, the best morphology and crystallinity were obtained at a slightly higher $\mathrm{V} /$ III ratio than that of InSb growth. This might be caused by the parasitic reaction between the TMSb and $\mathrm{AsH}_{3}$.

The composition dependence on the growth temperature was also investigated. When the temperature increased, the Sb composition was decreased. (Tab. 4.2). It might be due to the fact that $\mathrm{AsH}_{3}$ decomposition has been increased as the temperature increased.

After the growth temperature and V/III ratio were optimized, the dependence of Sb composition on the group $\mathrm{V}$ partial pressure ratio was studies. The growth temperature was set at $465{ }^{\circ} \mathrm{C}$ and the $\mathrm{V} / \mathrm{IIII}$ ratio was 12.5 . Group $\mathrm{V}$ input partial pressure ratio $\mathrm{TMSb} /(\mathrm{TMSb}+$ $\mathrm{AsH}_{3}$ ) has been varied from 0.67 to 0.73 , which resulted in the $\mathrm{Sb}$ composition from 0.65 to 0.80 . The result is plotted in Figure 4.2.

The X-ray diffraction peaks of InAsSb were broader than those of InSb of comparable thicknesses despite the good morphology of InAsSb films. The broadness is probably due to a large number of misfit dislocations and some compositional variations in the epitaxial layers. Figure 4.3 shows the X-ray diffraction of a $2 \mu \mathrm{m}$ thick $\operatorname{InAs}_{0.3} \mathrm{Sb}_{0.7}$ layer directly grown on GaAs substrate.

The dependence of X-ray FWHM on the Sb composition was studied. About $1.2 \mu \mathrm{m}$ thick samples with compositions varied from 0.56 to 0.80 were grown. The X-ray FWHM of the samples with $x=0.80$ were $\sim 1200$ arcs, which increased to $\sim 2000$ arcs with $x=0.56$. The peaks are broader than those of InSb films with comparable thicknesses and the more As is incorporated the broader 
Table 4.1. Physical properties of InAsSb [4].

\begin{tabular}{|c|c|c|c|c|}
\hline & $T(\mathrm{~K})$ & InAs & $\operatorname{InAs}_{0.35} \mathrm{Sb}_{0.65}$ & $\mathrm{InSb}$ \\
\hline Crystal structure & & Cub (ZnS) & Cub (ZnS) & $\mathrm{Cub}(\mathrm{ZnS})$ \\
\hline Lattice constant $(\AA)$ & 300 & 6.0584 & 6.36 & 6.47877 \\
\hline Coef. of thermal expansion & 300 & 5.02 & & 5.04 \\
\hline$\left(10^{-6} \mathrm{~K}^{-1}\right)$ & 80 & & & 6.50 \\
\hline Density $\gamma\left(\mathrm{g} / \mathrm{cm}^{3}\right)$ & 300 & 5.68 & & 5.7751 \\
\hline Melting point $(\mathrm{K})$ & & 1210 & & 803 \\
\hline \multirow[t]{3}{*}{ Energy gap $(\mathrm{eV})$} & 4.2 & 0.42 & 0.138 & 0.2357 \\
\hline & 80 & 0.414 & 0.136 & 0.228 \\
\hline & 300 & 0.359 & 0.1 & 0.180 \\
\hline Thermal coefficient of $E_{g}$ & $100-300$ & $-2.8 \times 10^{4}$ & & $-2.8 \times 10^{4}$ \\
\hline \multirow[t]{2}{*}{$m_{e}^{*} / m_{0}$} & 4.2 & 0.023 & & 0.0145 \\
\hline & 300 & 0.022 & 0.0101 & 0.0116 \\
\hline$m_{l h}^{*} / m_{0}$ & 4.2 & 0.026 & & 0.0149 \\
\hline$m_{h h}^{*} / m_{0}$ & 4.2 & 0.43 & 0.41 & 0.41 \\
\hline $\begin{array}{l}\text { Momentum matrix element } P \\
(\mathrm{~cm} 2 / \mathrm{V} \mathrm{s})\end{array}$ & & $9.2 \times 10^{-8}$ & & $9.4 \times 10^{-8}$ \\
\hline \multirow[t]{2}{*}{ Electron mobility $\left(\mathrm{cm}^{2} / \mathrm{Vs}\right)$} & 300 & $3 \times 10^{4}$ & $5 \times 10^{5}$ & $8 \times 10^{4}$ \\
\hline & 77 & $8 \times 10^{4}$ & $5 \times 10^{4}$ & $10^{6}$ \\
\hline \multirow[t]{2}{*}{ Hole mobility $\left(\mathrm{cm}^{2} / \mathrm{Vs}\right)$} & 300 & 500 & & 800 \\
\hline & 77 & & & $10^{4}$ \\
\hline \multirow[t]{3}{*}{ Intrinsic carrier conc. $\left(\mathrm{cm}^{-3}\right)$} & 77 & $6.5 \times 10^{3}$ & $2.0 \times 10^{12}$ & $2.6 \times 10^{9}$ \\
\hline & 200 & $7.8 \times 10^{12}$ & $8.6 \times 10^{15}$ & $9.1 \times 10^{14}$ \\
\hline & 300 & $9.3 \times 10^{14}$ & $4.1 \times 10^{16}$ & $1.9 \times 10^{16}$ \\
\hline Refractive index & & 3.44 & & 3.96 \\
\hline Static dielectric constant & & 14.5 & & 17.9 \\
\hline High frequency dielectric constant & & 11.6 & 16.8 & \\
\hline \multicolumn{5}{|l|}{ Optical phonon $\left(\mathrm{cm}^{-1}\right)$} \\
\hline $\mathrm{LO}$ & & 242 & $\gg 220$ & 193 \\
\hline TO & & 220 & $\gg 200$ & 185 \\
\hline
\end{tabular}

the peaks. As InAsSb is less lattice-mismatched to GaAs than InSb, this behavior can be explained by the variation in the alloy composition, which becomes more significant as the composition of As is increased.

For monolithic integration applications, growth of InAsSb layers on GaAs-coated Si substrates was also carried out. InAsSb layers were grown on both GaAs/Si and GaAs substrates in the same run. X-ray diffraction spectra was measured. From the comparison of the epilayers on two substrates, it's confirmed that the qualities of the InAsSb layers are about the same. Figure 4.5 shows the $\mathrm{X}$-ray diffraction spectra of InAsSb/InSb layer grown on GaAs/Si substrates. It demonstrated that the high quality InAsSb layer can be grown on GaAs/Si substrates.

\subsubsection{Electrical characteristics}

Normally undoped InAsSb layer showed negative Hall coefficient through entire temperature form 4 to $300 \mathrm{~K}$. To
Table 4.2. Dependence of Sb composition on the growth temperature at $\mathrm{V} / \mathrm{III}$ ratio of 12.5 .

\begin{tabular}{cc}
\hline Growth Temperature $\left({ }^{\circ} \mathrm{C}\right)$ & Sb composition $x$ \\
\hline 457 & 0.73 \\
466 & 0.68 \\
483 & 0.60 \\
\hline
\end{tabular}

investigate the electrical properties of the films with different $\mathrm{Sb}$ composition, $2 \mu \mathrm{m}$ thick layer with different $\mathrm{Sb}$ compositions have been grown. The electrical properties of the samples were measured at $77 \mathrm{~K}$ sand $300 \mathrm{~K}$.

The carrier concentrations at $77 \mathrm{~K}$ ranged from 1 to $6 \times 10^{16} \mathrm{~cm}^{-3}$ and $x$ around 0.65 gives the highest value (Fig. 4.6). This result is in good agreement with the previous result obtained by $\mathrm{Y}$ en [25] and is reasonable since InAsSb has lowest bandgap at $x \sim 0.65$. The Hall mobility was from 4000 to $15000 \mathrm{~cm}^{2} / \mathrm{Vs}$ decreasing as the 


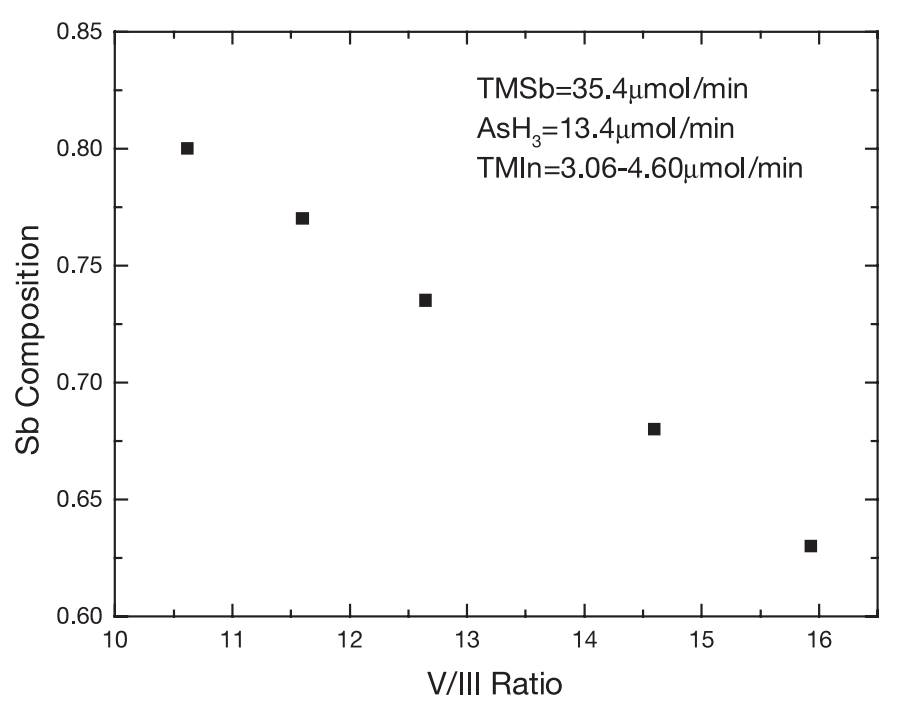

Fig. 4.1. Dependence of Sb composition in the InAsSb epilayer on V/III ratio at a growth temperature of $465{ }^{\circ} \mathrm{C}$.

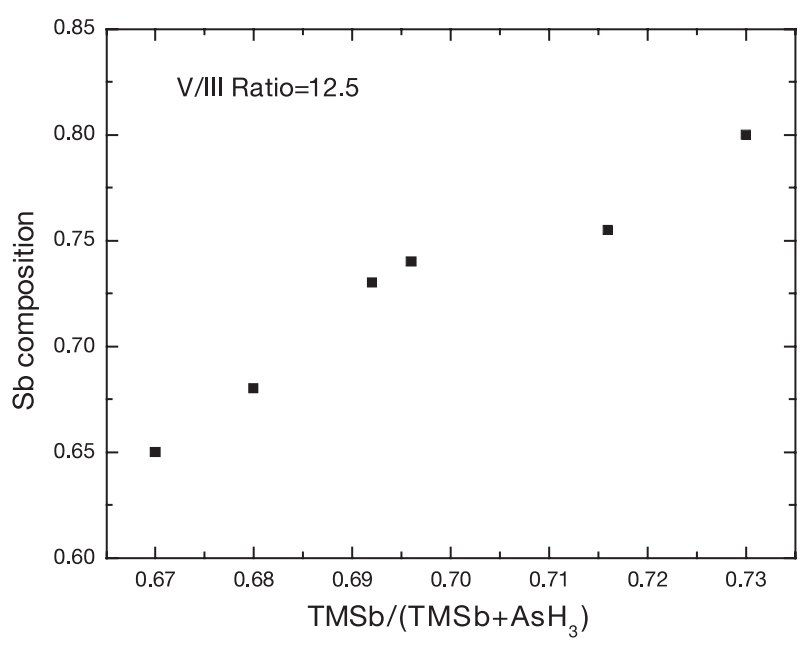

Fig. 4.2. Dependence of Sb composition on the group V fraction of TMSb in the vapor phase.

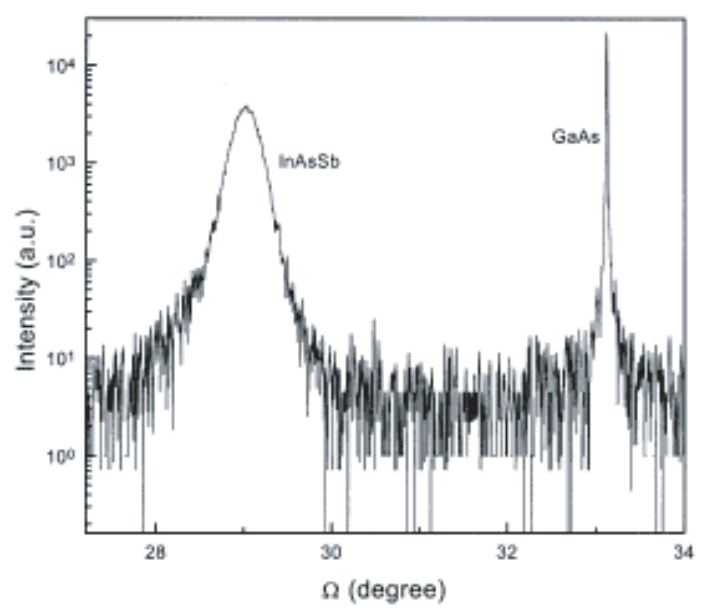

Fig. 4.3. X-ray diffraction spectra of $2 \mu \mathrm{m} \mathrm{InAs}_{0.3} \mathrm{Sb}_{0.7}$ layer grown on GaAs substrate.

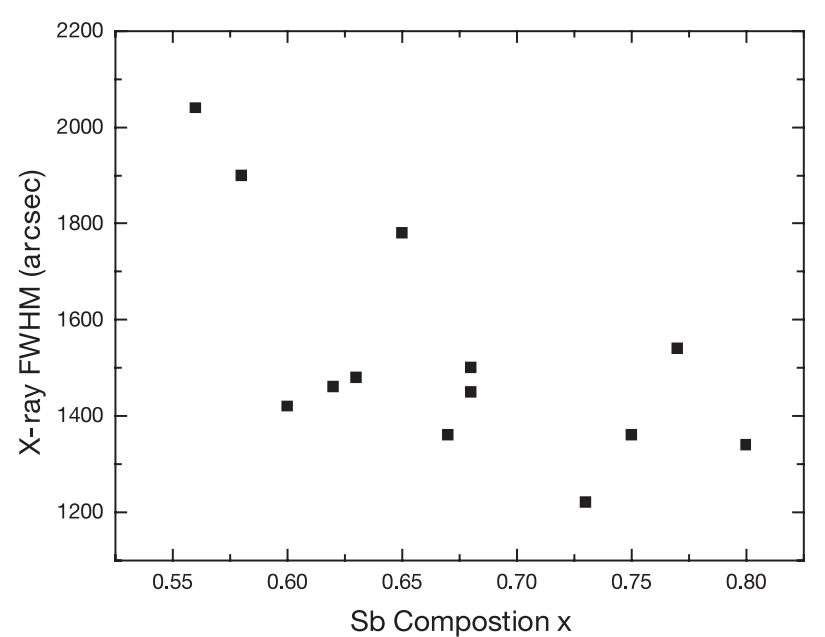

Fig. 4.4. Dependence of X-ray FWHM of $\operatorname{InAs}_{1-x} \mathrm{Sb}_{x}$ epilayer on $x$.

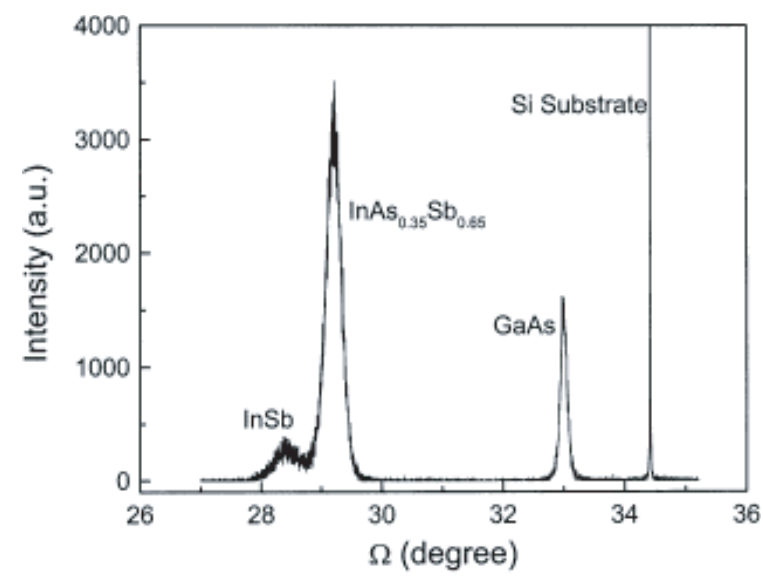

Fig. 4.5. X-ray diffraction spectra of InAsSb on GaAs/Si substrates.

As composition is increasing, as shown in Figure 4.7. Similar behavior has been found by Woolley et al. [26] and Tansley et al. [27] and expatiation were given by the effect of alloy scattering.

At $300 \mathrm{~K}$, carrier concentration increased to $4-14 \times 10^{16} \mathrm{~cm}^{-3}$ and Hall mobility increased to 5000 $30000 \mathrm{~cm}^{2} / \mathrm{Vs}$. The dependence of both properties on the Sb composition $x$ was similar to that at $77 \mathrm{~K}$. It becomes more clear at $300 \mathrm{~K}$ that the carrier concentration is dominated by the intrinsic concentration and is peaked at $x \sim 0.65$. The Hall mobility should increase as the temperature goes down, which is the opposite to our experimental results. The strange behavior suggested that the InAsSb layer could be $p$-type. Theoretical calculations on the $\mathrm{InAs}_{0.3} \mathrm{Sb}_{0.7}$ layers have been performed to verify this behavior. It showed that with a shallow $p$-type background doping density of $5 \times 10^{15} \mathrm{~cm}^{-3}$ and a dislocation density in the order of $2.5-5 \times 10^{8} \mathrm{~cm}^{-3}$, calculated data could well fit the experimental data [28]. 


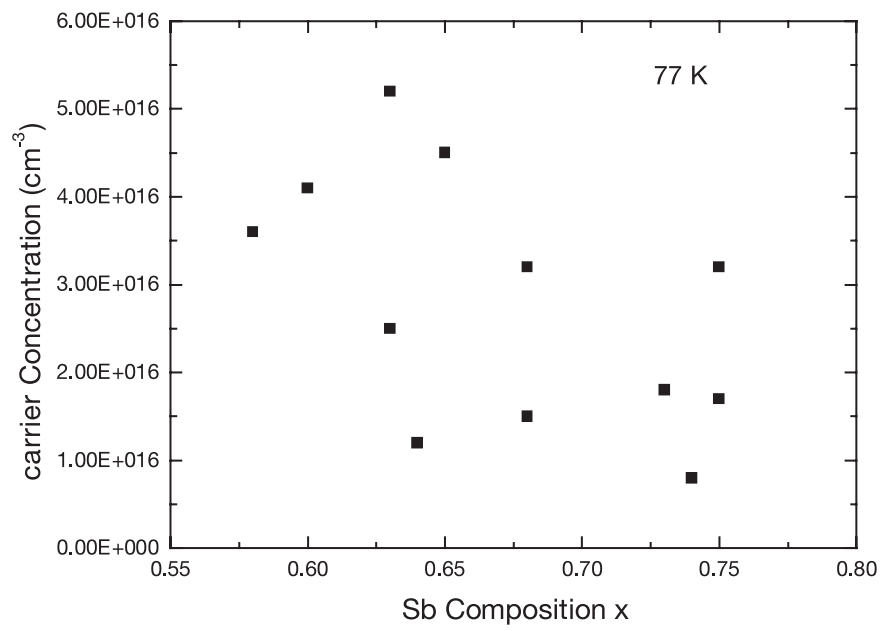

Fig. 4.6. Dependence of $77 \mathrm{~K}$ carrier concentration of $\operatorname{InAs}_{1-x} \mathrm{Sb}_{x}$ layer on $x$.

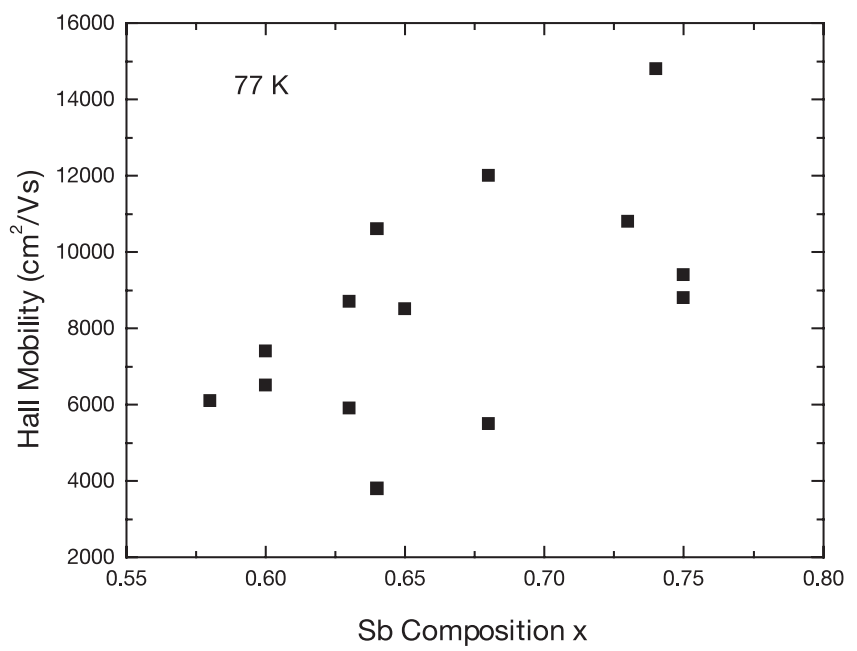

Fig. 4.7. Dependence of $77 \mathrm{~K}$ Hall mobility of $\operatorname{InAs}{ }_{1-x} \mathrm{Sb} x$ layer on Sb composition $x$.

\subsubsection{Optical characteristics}

Optical characterizations on the InAsSb layers have been performed using FTIR. Bandgap variation with As composition can be clearly observed. Optical bandgap can be determined in two ways. First, the normalized transmission spectra is used to calculate the absorption coefficient of the layer. Usual definition of the optical bandgap for the narrow gap semiconductors is the photon energy for which absorption coefficient is equal to $500 \mathrm{~cm}^{-1}$. Figure 4.8 shows the transmission spectra of InAsSb layer with different compositions and thickness at $300 \mathrm{~K}$. Bandgap of $103 \mathrm{meV}$ was derived for $\operatorname{InAs}_{1-x} \mathrm{Sb}_{x}$ with $x=0.77$. Even narrower bandgap of $83 \mathrm{meV}$ was obtained for $x=0.65$. This shows that InAsSb can be used for the near room temperature photodetectors operating in the entire $8-14 \mu \mathrm{m}$ range.

To obtain more accurate absorption coefficient value, the InAsSb layer was successively etched and transmission

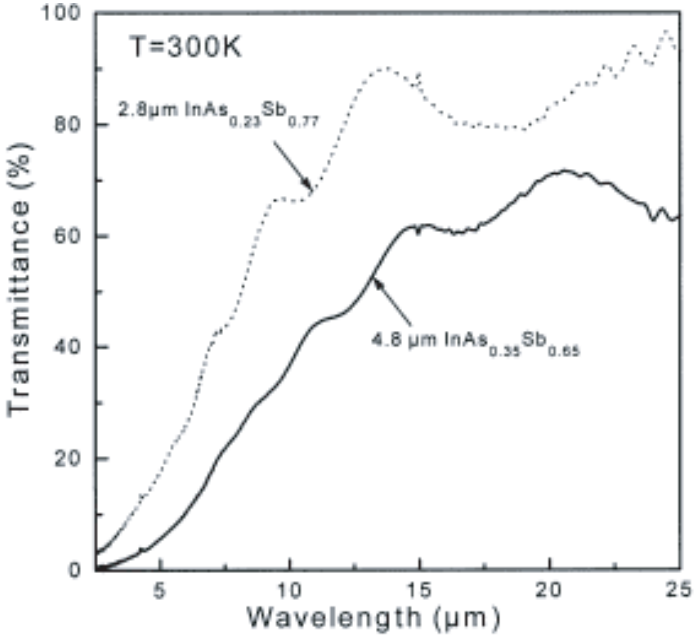

Fig. 4.8. Transmission spectra of InAsSb films with different compositions.

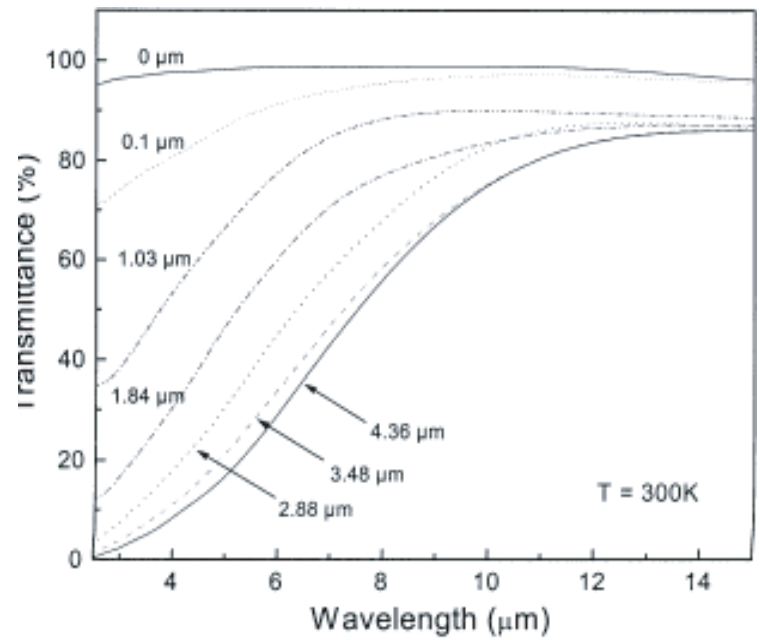

Fig. 4.9. Transmission spectra of InAsSb layer at different thicknesses.

spectra were measured at different thicknesses. The transmission spectra were then smoothed out to remove both the high frequency noise and the Fabry-Perot interference fringes. Figure 4.9 shows the smoothed transmission spectra.

The relationship between the transmittance $T$ and the absorption coefficient $\alpha$ at a certain wavelength is expressed as

$$
T=\mathrm{e}^{-\alpha t}
$$

where $t$ is the thickness of the layer. For certain wavelength, $T$ can be plotted as a function of thickness $t$ by numerical fitting, $\alpha$ can be determined. In this method, $\alpha$ is determined to be $1330 \mathrm{~cm}^{-1}$ for wavelength of $8 \mu \mathrm{m}$.

More accurate measurements of the optical bandgap has been done using the photoconductivity spectra. The optical bandgap of the narrow gap semiconductors can be determined as the energy at which the normalized photoresponse falls to $20 \%$ of the maximum value as shown in Figure 4.10. 


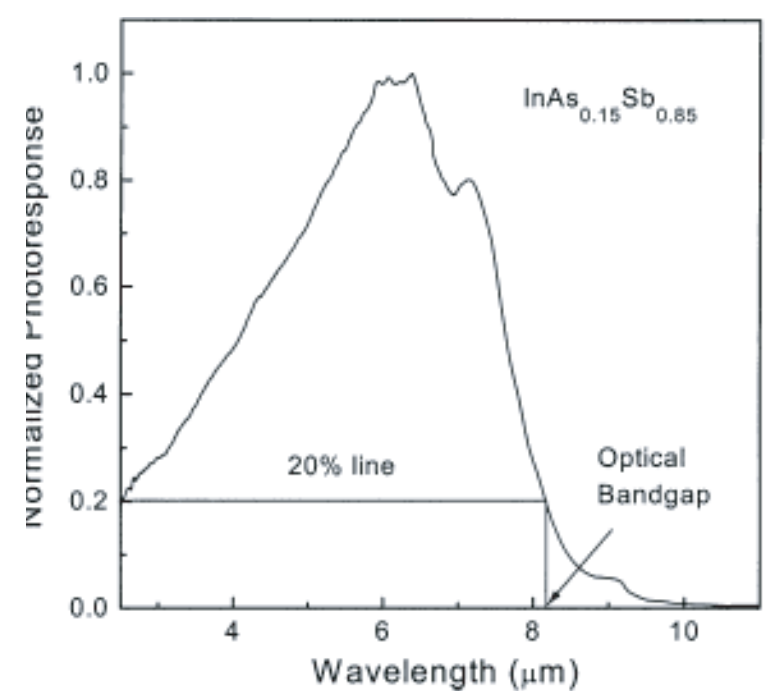

Fig. 4.10. Determination of optical bandgap using the normalized photoresponse spectra.

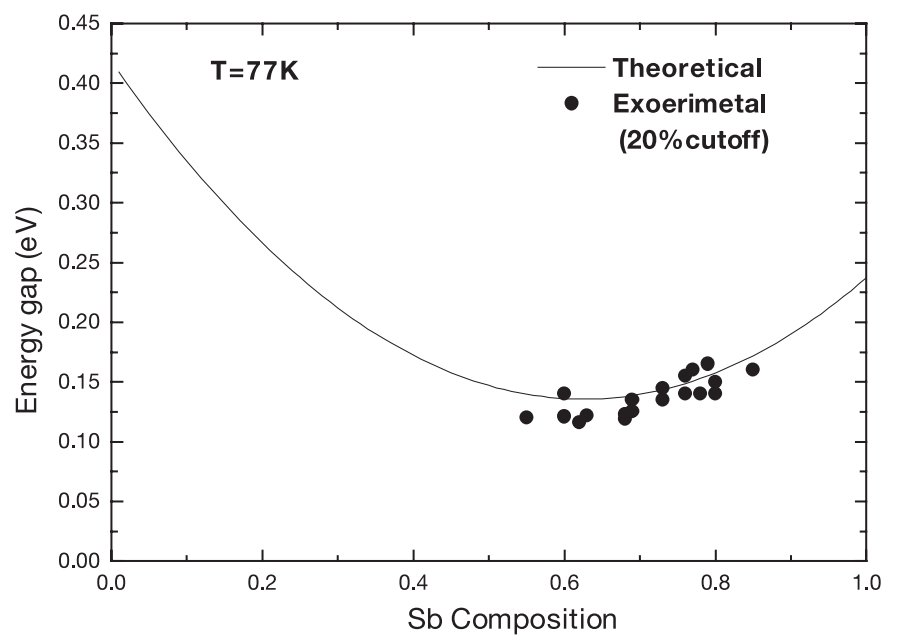

Fig. 4.11. Comparison of experimental optical bandgap with theoretical data for $\operatorname{InAs}_{1-x} \mathrm{Sb}_{x}$ with different compositions.

InAs $\mathrm{A}_{1-x} \mathrm{Sb}_{x}$ with different compositions have been grown and the optical bandgap have been determined using the normalized photoresponse spectra. Figure 4.11 shows both the theoretical and experimental bandgap at $77 \mathrm{~K}$. the experimental values are lower than the theoretical, which has been attributed to the long range ordering.

\subsubsection{Device measurement}

\subsubsection{1 $\ln \mathrm{AsSb}$ photoconductors}

Photoconductors based on $p$-type $\operatorname{InAs}_{0.23} \mathrm{Sb}_{0.77}$ were grown on GaAs substrates by LP-MOCVD [29]. The photoconductor structure composed of two epitaxial layers ( $p$ $\mathrm{InAs}_{0.23} \mathrm{Sb}_{0.77} / p$-InSb) grown on GaAs. InSb is used as a buffer layer with $2 \%$ lattice-mismatch to $\operatorname{InAs}_{0.23} \mathrm{Sb}_{0.77}$ and also as a confinement layer for the electrons in the

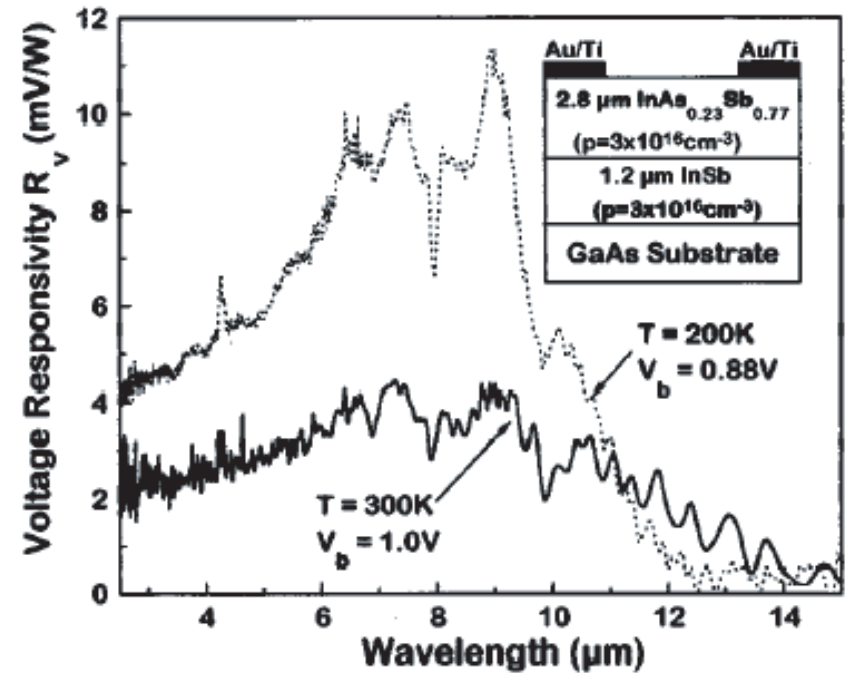

Fig. 4.12. Spectral response of the $\mathrm{InAs}_{0.23} \mathrm{Sb}_{0.77}$ photoconductor at 200 and $300 \mathrm{~K}$.

active layer. The optimized doping level for room temperature operation is around $3 \times 10^{16} \mathrm{~cm}^{-3}$. The epitaxial layers have been grown on (100) semi-insulating GaAs substrates at a growth temperature around $470{ }^{\circ} \mathrm{C}$. The geometry of the photoconductor is $4 \times 3 \mathrm{~mm}^{2}$. Au/Ti contacts were deposited by an e-beam evaporator. The resistance is $23 \Omega$ at $300 \mathrm{~K}$ and $220 \Omega$ at $200 \mathrm{~K}$.

The photodetectors were mounted in a liquid nitrogen cooled cryostat system and measurements were taken at temperatures between $77 \mathrm{~K}$ and $300 \mathrm{~K}$. The relative photoresponse spectra were measured with an FTIR spectrometer. Then the responsivity was calibrated by a blackbody test setup, including a back body source (Mikron 305), preamplifier (EG\&G PA-6), and chopper system (Stanford Research system SR540). The blackbody temperature is set at $800 \mathrm{~K}$. the modulation frequency is set at $450 \mathrm{~Hz}$ since response measurements as a function of chopper frequency showed that the thermal effect could be neglected at frequencies higher than $200 \mathrm{~Hz}$.

Figure 4.12 shows the spectral responsivities of a photoconductor sample at 200 and $300 \mathrm{~K}$. The peak responsivity is about $4 \mathrm{mV} / \mathrm{W}$ at $8 \mu \mathrm{m}$ and at $300 \mathrm{~K}$ for $1 \mathrm{~V}$ bias. The cut-off wavelength around $14 \mu \mathrm{m}$ was observed at $300 \mathrm{~K}$. Noise in the spectral response was attributed to FTIR preamplifier since the contribution of thermal or bolometric response is negligible at measuring frequencies of FTIR which are higher than $1.2 \mathrm{kHz}$. Figure 4.13 displays the voltage-dependent responsivity at $10.6 \mu \mathrm{m}$. It increases with applied voltage and reaches saturation at around $3 \mathrm{~V}$, which corresponds to the values of $5.8 \mathrm{mV} / \mathrm{W}$ at around $3 \mathrm{~V}$. The corresponding Johnson-noise limited detectivity of the photoconductor was then estimated to be $3.27 \times 10^{7} \mathrm{~cm} \mathrm{~Hz}^{1 / 2} / \mathrm{W}$ at $300 \mathrm{~K}$.

Based on the spectral voltage responsivity, the mobility-effective carrier lifetime product $\mu_{e} \tau$ and the effective lifetime $\tau$ can be derived. According to the 


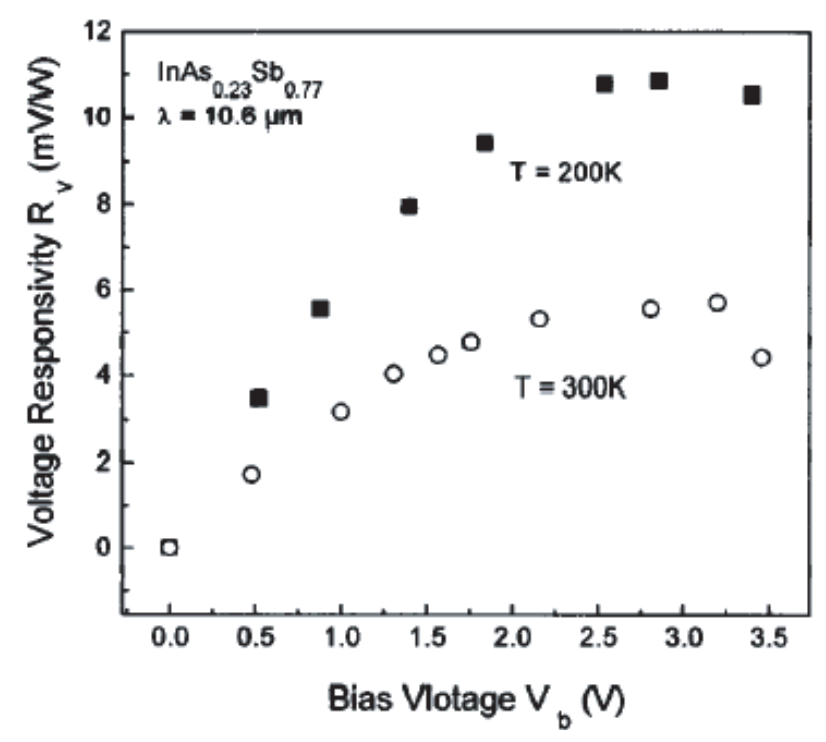

Fig. 4.13. Voltage-dependent responsivity of the $\mathrm{InAs}_{0.23} \mathrm{Sb}_{0.77}$ photoconductor at $10.6 \mu \mathrm{m}$.

simple theory of photoconductivity, the voltage responsivity $R_{V}$ is

$$
R_{V}=\frac{q \lambda}{h c} \frac{\eta \mu_{e} \tau V_{b} R_{D}}{L^{2}}\left(1+\frac{1}{b}\right)
$$

where $q$ is the electron charge, $\lambda$ is the wavelength, $\eta$ is the quantum efficiency, $b$ is the electron-to-hole mobility ratio, $L$ is the detector length, $V_{b}$ is the bias voltage, and $R_{D}$ is the detector resistance. The quantum efficiency $\eta$ is given by

$$
\eta=(1-r) \frac{\left(1-\mathrm{e}^{-\alpha t}\right)}{1-r \mathrm{e}^{-\alpha t}}
$$

where $r$ is the reflectance, $\alpha$ is the absorption coefficient, and $t$ is the thickness of the layer. If $\mu_{e}$ is known, $\tau$ can be estimated. Based on the electron mobility at $300 \mathrm{~K}$, the effective lifetime is estimated to be $0.14 \mathrm{~ns}$.

\subsubsection{2 $\ln \mathrm{AsSb}$ photodiodes}

For the realization of InAsSb photovoltaic detectors, $p^{+}$ $\mathrm{InSb} / p$-InAs $\mathrm{As}_{1-x} \mathrm{Sb}_{x} / \mathrm{n}^{+}-\mathrm{InSb}$ double heterojuction structures were grown on semi-insulating GaAs substrates by MOCVD [30]. The growth temperature was kept at $470{ }^{\circ} \mathrm{C}$ and the V/III ratio was $\sim 20$. The growth rate was $\sim 0.7-$ $0.9 \mu \mathrm{m} / \mathrm{h}$. The standard structure consisted of a $2 \mu \mathrm{m}$ heavily doped $n$-type InSb layer $\left(\sim 3 \times 10^{18} \mathrm{~cm}^{-3}\right)$, a $5 \mu \mathrm{m}$ $p$-type $\left(3.6 \times 10^{16} \mathrm{~cm}^{-3}\right) \mathrm{InAs}_{1-x} \mathrm{Sb}_{x}$ absorber region, and a $0.5 \mu \mathrm{m}$ heavily doped InSb $p$-type $\left(\sim 3 \times 10^{18} \mathrm{~cm}^{-3}\right)$ contact layer.

Structural characterization was performed using X-ray diffraction spectra at (004) orientation. The composition of $\mathrm{InAs}_{1-x} \mathrm{Sb}_{x}$ has been determined from X-ray diffraction data and Vegard's law. Figure 4.14 shows a typical $\mathrm{X}$-ray diffraction spectrum of an InAsSb/InSb/GaAs heterostructure. Peaks for InSb and $\mathrm{InAs}_{1-x} \mathrm{Sb}_{x}$ are clearly resolved and have relatively low FWHM $(\sim 300$ arcs for

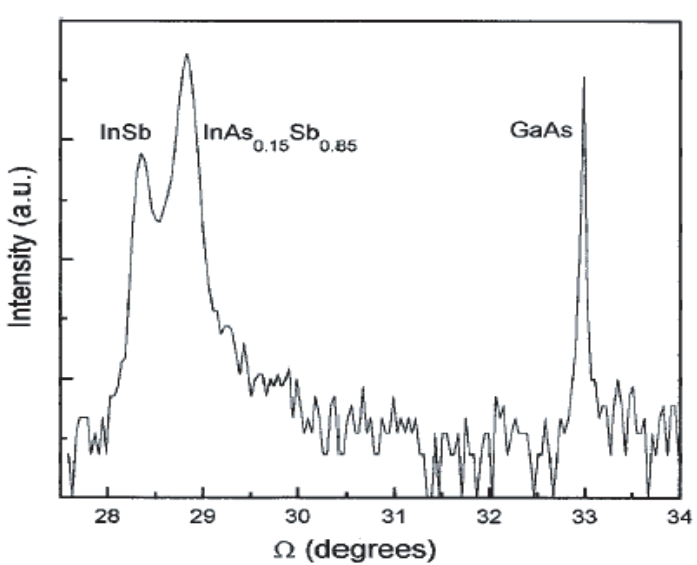

Fig. 4.14. X-ray diffraction of the heterojunction.

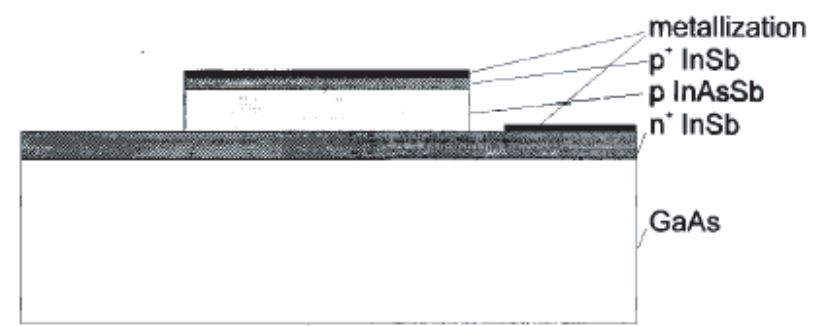

Fig. 4.15. Schematic representation of the photodiode structure.

both InSb and InAsSb), indicating high-quality material. The InAs ${ }_{1-x} \mathrm{Sb}_{x}$ composition calculated from diffraction data was $x=0.85$. Figure 4.15 shows the geometry of the photovoltaic device. The mesa structure has been etched using photolithography. $\mathrm{Au} / \mathrm{Pt} / \mathrm{Ti}$ contacts were deposited by an e-beam evaporator.

Figure 4.16 shows the voltage response at various temperatures. The temperature dependent cut-off wavelength is clearly observed. Photoresponse up to $13 \mu \mathrm{m}$ has been observed. The peak voltage-responsivity is $9.13 \times$ $10^{-2} \mathrm{~V} / \mathrm{W}$ at $300 \mathrm{~K}$. However it is only $2.85 \times 10^{1} \mathrm{~V} / \mathrm{W}$ at $77 \mathrm{~K}$, which is much lower than the expected value. Possible reasons are the poor interface properties due to the lattice mismatch between the absorber and contact layers and high dark current due to the high doping level in the active layer.

As the series resistance is much higher than the junction resistance at high temperature, we could not measure the resistance-area product $R_{0} A$ directly from the $I-V$ curve [30]. The $R_{0} A$ product can be calculated,

$$
R_{v} A=\frac{\lambda \eta q R_{0} A}{h c}
$$

where $\lambda$ is the wavelength, $\eta$ is the quantum efficiency, $q$ is the electron charge, $h$ is the Planck constant, and $c$ is the light velocity. Table 4.3 shows the responsivity-area product and resistance-area product of the $\operatorname{InAs}_{0.15} \mathrm{Sb}_{0.85}$ photodiode at various temperatures [31].

At high temperatures, the $R_{o} A$ products were close to the value set by Auger-limited process, but they were far below the calculated values at low temperatures, as shown 


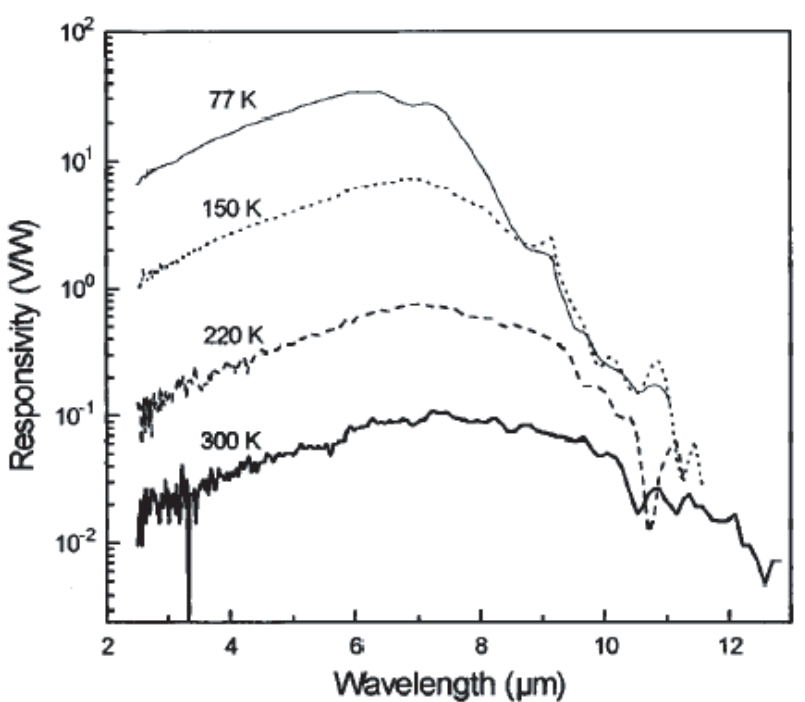

Fig. 4.16. Spectral response of InAsSb photovoltaic detector at different temperatures.

Table 4.3. Peak voltage responsivity-area product and corresponding resistance-area product of InAsSb photovoltaic detectors at various temperatures.

\begin{tabular}{ccc}
\hline$T(\mathrm{~K})$ & $R_{v} A\left(\mathrm{~V} \mathrm{~cm}^{2} / \mathrm{W}\right)$ & $R_{o} A\left(\Omega \mathrm{cm}^{2}\right)$ \\
\hline 77 & $4.56 \times 10^{-2}$ & $3.15 \times 10^{-2}$ \\
100 & $2.58 \times 10^{-2}$ & $1.53 \times 10^{-2}$ \\
150 & $9.68 \times 10^{-3}$ & $5.73 \times 10^{-3}$ \\
200 & $1.64 \times 10^{-3}$ & $9.71 \times 10^{-4}$ \\
220 & $1.03 \times 10^{-3}$ & $6.10 \times 10^{-4}$ \\
250 & $5.57 \times 10^{-4}$ & $3.30 \times 10^{-4}$ \\
300 & $1.46 \times 10^{-4}$ & $7.56 \times 10^{-5}$ \\
\hline
\end{tabular}

in the plot in Figure 4.17. The $R_{o} A$ products at low temperatures are limited by generation-recombination process. It is evident from the plot of the experimental data that $R_{o} A$ is limited by generation-recombination processes but with different carrier lifetimes. As is discussed before, the difference in the carrier lifetime maybe be caused by poor heterointerface properties, surface recombination, or Shockley-Read recombination in the active region.

Further increase in the responsivity of the photovoltaic detectors has been achieved by biasing the device. As shown in Figure 4.18, the voltage responsivity of the InAsSb photovoltaic detectors at near room temperatures has been increased by a factor of $\sim 3$ at $0.4 \mathrm{~V}$ reverse bias. This shows the possible improvement in the device performance through non-equilibrium mode operation.

\subsection{MBE growth of InAsSb, AllnSb and GalnSb}

As described before, there is a great amount of work in IR detector material covering 8-12 $\mu \mathrm{m}$ wavelength region. However, one application that requires cutoff wavelength around $8 \mu \mathrm{m}$ is the projectile fuze. Due to the absorption

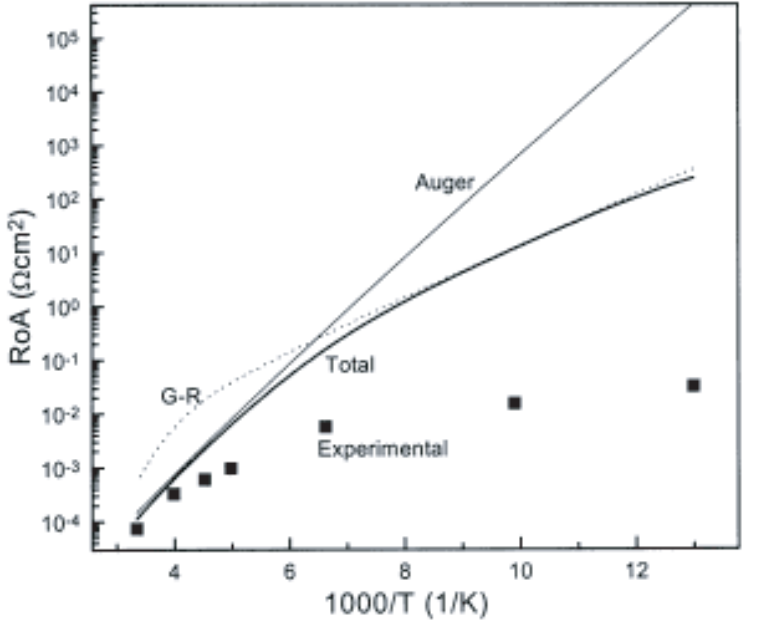

Fig. 4.17. Comparison of experimental $R_{o} A$ values with the theoretical limit.

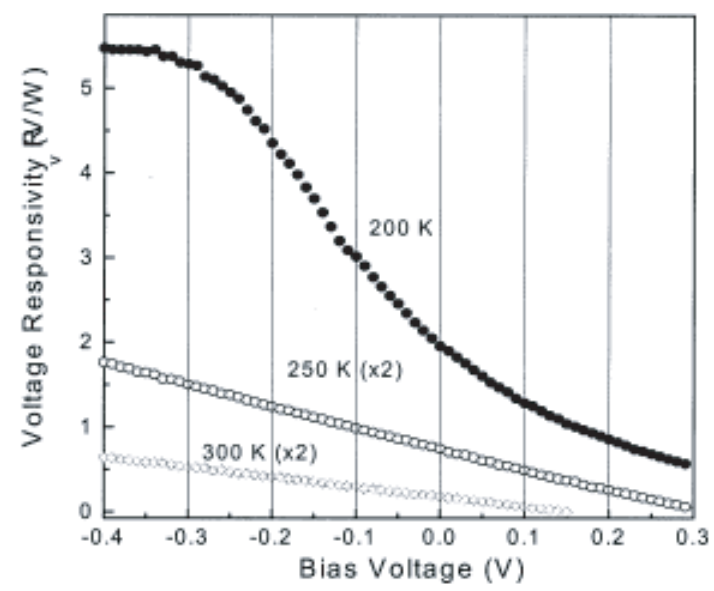

Fig. 4.18. Bias dependent voltage responsivity of the In $\mathrm{As}_{0.15} \mathrm{Sb}_{0.85}$ photovoltaic detectors.

in the atmosphere in the $5-8 \mu \mathrm{m}$ region, the projectile fuze doesn't detect a hot object until it is in close proximity.

\subsubsection{Growth and characterizations}

The growth of InAsSb, especially for high Sb composition, is very similar to the growth of InSb. The layers were grown on GaAs substrate using an EPI Modular Gen II Solid source MBE system with a $\mathrm{V} / \mathrm{III}$ ratio of $\geq 1.2$. The composition was controlled by RHEED calibration of the In and $\mathrm{Sb}$ cell, and using an overpressure of As, the substrate temperature was varied to obtain correct composition, which resulted in an optimum temperature of $\sim 400{ }^{\circ} \mathrm{C}$ as determined by IR pyrometer.

Although InAsSb was studied for As compositions from 0 to $35 \%$, the work described here focused on $\sim 5 \%$ As content for the projectile fuze applications. Nevertheless, some results shown below are for the entire range.

Generally, as the As composition increased from InSb, the X-ray FWHM increases, as shown in Figure 4.19. The mobility of InAsSb layers was less than InSb, decreasing 


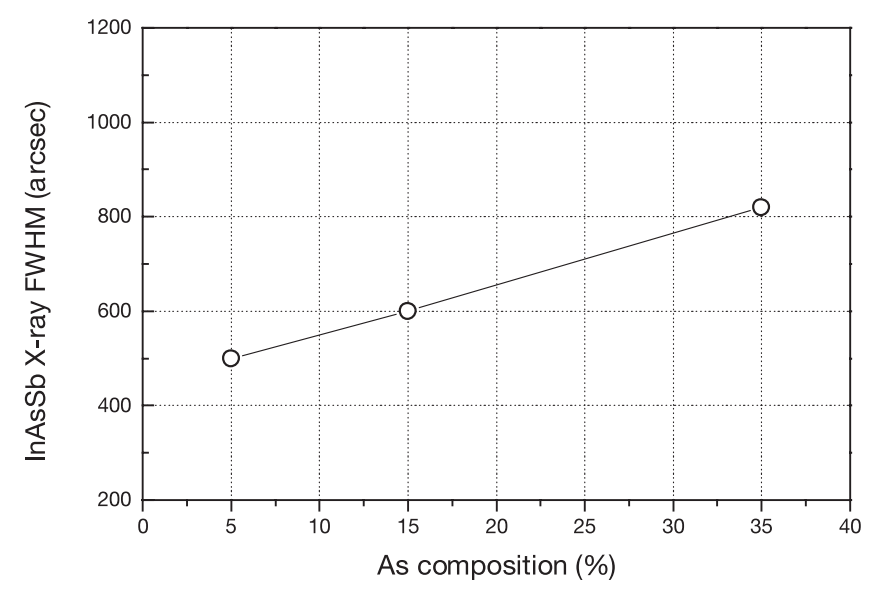

Fig. 4.19. InAsSb X-ray FWHM versus As composition.

with increasing As content. Room temperature mobility was in the $5000-50000 \mathrm{~cm}^{2} / \mathrm{V} \mathrm{s}$ range, and was in the few thousand $\mathrm{cm}^{2} / \mathrm{Vs}$ at $77 \mathrm{~K}$. Photoluminescence measurements were performed on samples. The PL linewidths at $77 \mathrm{~K}$ were in the $30-60 \mathrm{meV}$ range.

In addition to InAsSb, AlInSb and GaInSb were investigated for these detector structures. These layers have wider bandgaps, so that carriers can be confined to the active region. Also, if doped properly, the layers can be barriers to one type of carrier while allowing the other type of carrier through to a contact.

\subsubsection{InAsSb photodiodes}

InAsSb photodiodes structures were grown on (100) and (111) GaAs substrates by MBE. Several device structures were investigated. First $p^{+}-\mathrm{InSb} / p-\mathrm{InAs}_{1-x} \mathrm{Sb}_{x} / n^{+}-\mathrm{InSb}$ double heterojunction structures were grown on semiinsulating GaAs substrates. The bottom $n^{+}$-InSb layer with $\sim 2 \mu \mathrm{m}$ thickness was doped at $2 \times 10^{18} \mathrm{~cm}^{-3}$ both for increased quantum efficiency due to the pronounced MossBurstein effect and for a low series resistance due to high electron mobility. The thickness of the active layer was $\sim 2-5 \mu \mathrm{m}$ to ensure both high quantum efficiency and high optical gain. The top $p^{+}$-InSb contact layer was $0.5 \mu \mathrm{m}$ thick and doped at a level of $10^{18} \mathrm{~cm}^{-3}$.

Some device structures utilized the bottom InSb layer as a buffer layer with the bottom contact made directly to the InAsSb active region. Subsequent devices utilized a wider gap AlInSb buffer (or bottom contact) layer. This structure gave the flexibility that the mesa could be etched down to the InAsSb active region or down to the AlInSb layer which resulted in a heterojunction.

A typical absolute photoresponse of an InAsSb photodiode on an InSb buffer layer is shown in Figure 4.20. The $300 \mathrm{~K}$ Johnson-noise limited detectivity for this device was calculated at $\sim 1 \times 10^{8} \mathrm{~cm} \mathrm{~Hz}^{1 / 2} / \mathrm{W}$. The $R_{o} A$ was $\sim 10^{-3} \Omega \mathrm{cm}^{2}$ at $300 \mathrm{~K}$, which is attributed to the highly dislocated interface layer and the unpassivated surface. The $R_{o} A$ was increased by an order of magnitude by incorporating an AlInSb buffer layer, which blocks carriers

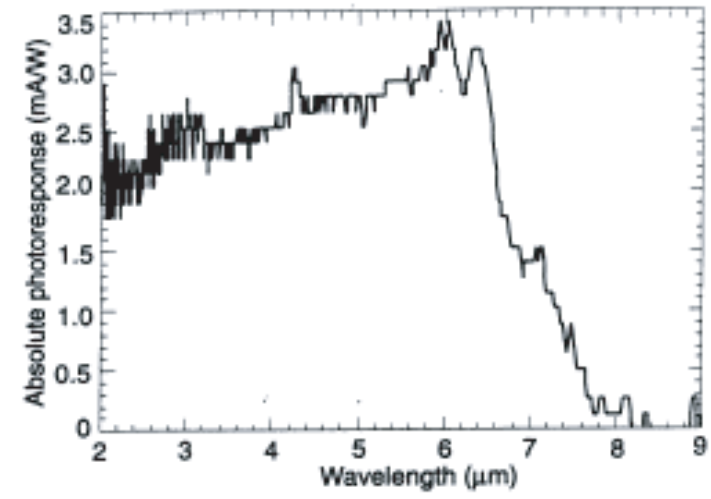

Fig. 4.20. Room temperature absolute photoresponse of an InAsSb photodiode on InSb/GaAs.

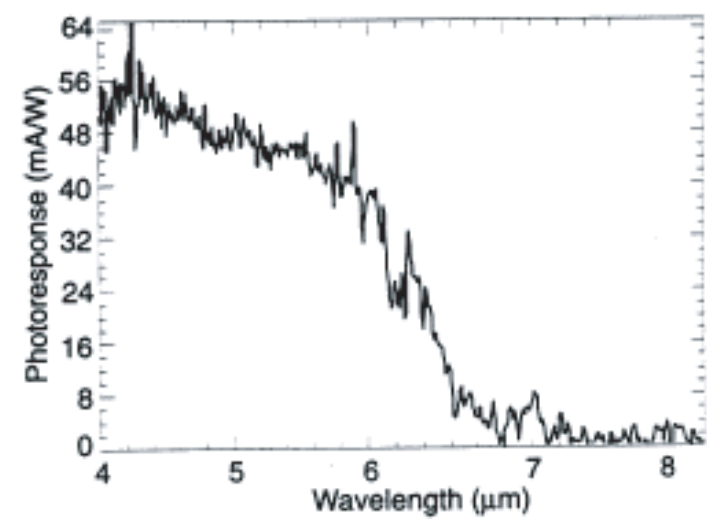

Fig. 4.21. Room temperature absolute photoresponse of an InAsSb photodiode on AlInSb/GaAs.

from the highly dislocated interface, resulting in a peak detectivity of $\sim 3 \times 10^{8} \mathrm{~cm} \mathrm{~Hz}^{1 / 2} / \mathrm{W}$. The absolute photoresponse for an InAsSb AlInSb homojunction on an AlInSb buffer layer is shown in Figure 4.21.

The wider gap AlInSb layer can also be used as the bottom contact layer resulting in a single heterostructure photodiode. The heterojuction increases the $R_{o} A$ of the device from $\sim 10^{-3} \Omega \mathrm{cm}^{2}$ to $\sim 10^{-1} \Omega \mathrm{cm}^{2}$. The current and differential resistance curves versus bias are shown for an InAsSb/AlInSb heterostructure device in Figure 4.22. The peak differential resistance of $70 \Omega$ at zero bias results in a $R_{o} A$ of $0.11 \Omega \mathrm{cm}^{2}$ at $300 \mathrm{~K}$. The absolute photoresponse of this device at $300 \mathrm{~K}$ is shown in Figure 4.23. The Johnson-noise limited detectivity was estimated at $\sim 4 \times 10^{8} \mathrm{~cm} \mathrm{~Hz}^{1 / 2} / \mathrm{W}$ at $3 \mu \mathrm{m}$ and $\sim 5 \times 10^{7} \mathrm{~cm} \mathrm{~Hz}^{1 / 2} / \mathrm{W}$ at $6 \mu \mathrm{m}$. The response curve shown was obtained under a small bias $(\sim 0.15 \mathrm{~V})$ since the device showed little or no response at zero bias. This may be due to the alignment of the band edges of InAsSb and AlInSb where electrons generated in the InAsSb region encounter a barrier at the $\mathrm{AlInSb} / \mathrm{InAsSb}$ interface.

\section{$5 \ln$ TISb}

As an alternative to the InAsSb system, InTlSb is also a potential material for $\lambda>8 \mu \mathrm{m}$ detectors. TlSb was 


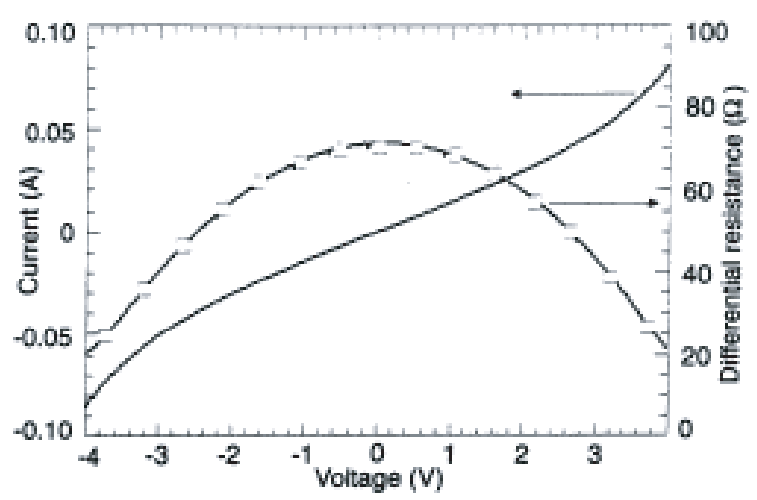

Fig. 4.22. Room temperature current and differential resistance versus bias of an InAsSb photodiode on AlInSb/GaAs.

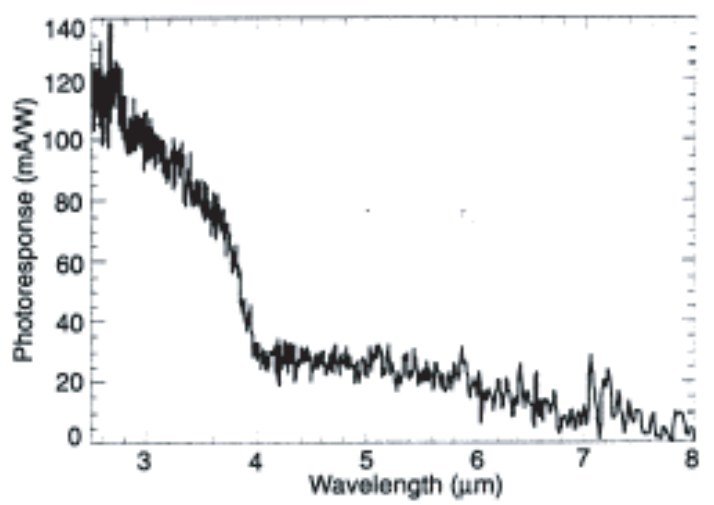

Fig. 4.23. Room temperature absolute photoresponse of an $\mathrm{InAsSb} / \mathrm{AlInSb}$ single heterostructure photodiode on GaAs.

calculated as a semimetal from the full-potential linear muffin-tin (LMTO) method within the local density approximation (LDA) [32]. By alloying TlSb with InSb, it was suggested that the band gap of InTlSb could be varied from $-1.5 \mathrm{eV}$ to $0.26 \mathrm{eV}$. Assuming a linear dependence of the band gap on alloy composition, InTlSb can then be expected to reach a band gap of $0.1 \mathrm{eV}$ at $x=0.08$ while exhibiting similar lattice constant as InSb since the radius of $\mathrm{Tl}$ atom is very similar to In. To date, there have been very limited experimental works reported on the growth and characterization of InTISb. Choi et al. reported on the first successful growth of InTlSb by LP-MOCVD [33]. A band gap shift toward a longer wavelength was observed, which suggest a concentration of $6 \% \mathrm{Tl}$. A group from University of Utah reported $\mathrm{Tl}$ incorporation of less than $1 \%$ in InSb [34]. Recently, Karam et al. reported the InTiSb films with Tl composition up to $10 \%$ [35]. Spectral response measurements showed photoresponse up to $14 \mu \mathrm{m}$ at $77 \mathrm{~K}$.

\subsection{MOCVD growth of InTISb}

The growth of InTlSb was performed on InSb, GaAs and GaAs-coated silicon substrate with a low pressure MOCVD. TMIn, TMSb and CPTl were used as precursors. The structure consisted of an InSb buffer layer grown
Table 5.1. Summery of growth conditions for the nominally undoped InTlSb layers.

\begin{tabular}{c|c|c}
\hline \multicolumn{2}{c|}{ Growth temperature } & $425{ }^{\circ} \mathrm{C}-475{ }^{\circ} \mathrm{C}$ \\
\hline \multicolumn{2}{c}{ Growth pressure } & 76 torr \\
\hline Substrate orientation & InSb, GaAs, GaAs-coated Si \\
\hline \multirow{2}{*}{ TMIn } & Temperature & $18{ }^{\circ} \mathrm{C}$ \\
\cline { 2 - 3 } & Flow rate & $\sim 36-55 \mathrm{sccm}$ \\
\hline \multirow{2}{*}{ TMSb } & Temperature & $0{ }^{\circ} \mathrm{C}$ \\
\cline { 2 - 3 } & Flow rate & $16-36 \mathrm{sccm}$ \\
\hline \multirow{2}{*}{$\mathrm{CPTl}$} & Temperature & $0-80{ }^{\circ} \mathrm{C}$ \\
\cline { 2 - 3 } & Flow rate & $3-300 \mathrm{sccm}$ \\
\hline $\mathrm{H}_{2}$ & Flow rate & $1.5 \mathrm{l} / \mathrm{min}$ \\
\hline
\end{tabular}

for one hour followed by an InTlSb layer grown for another hour. InSb buffer layers were grown under the optimum conditions determined from InSb investigations. The growth of InTlSb was first attempted by introducing a small flow of CPTl without changing other growth parameters. Once the growth of InTlSb has been verified, different amount of $\mathrm{Tl}$ was sent in order to obtain $\mathrm{In}_{1-x} \mathrm{Tl}_{x} \mathrm{Sb}$ alloys of various $x$ compositions. This was achieved by varying both the bubbler temperature as well as the flow rate of CPTl. Table 5.1 summarizes the growth conditions for InTlSb.

\subsubsection{Chemical analysis by Auger electron spectroscopy}

Auger electron spectroscopy measurement was performed on InTlSb samples and compared with that of reference $\mathrm{InSb}$ to verify the incorporation of $\mathrm{Tl}$ [36]. Figure 5.1 shows the Auger electron spectrum of an InTiSb sample as well as that of a reference InSb. The InTlSb sample shows a noticeable intensity dip near $70 \mathrm{eV}$ compared with the reference sample, confirming the presence of $\mathrm{Tl}$.

\subsubsection{Structural characteristics}

X-ray diffraction measurements were performed on as grown InTlSb samples. Tl flow was varied to grow InTlSb alloys with different Tl composition [37-40]. Figure 5.2 shows high resolution (004) X-ray diffraction curves of the $\mathrm{In}_{1-x} \mathrm{Tl}_{x} \mathrm{Sb}$ layers grown on GaAs substrates. An InSb epilayer is grown on the GaAs substrate to provide a reference peak for the X-ray diffraction study. The X-ray peaks corresponding to the InSb epilayer and GaAs substrate are observed at an angle of $28.39^{\circ}$ and $33.02^{\circ}$, respectively. A noticeable feature appeared in the X-ray diffraction spectra for the $\mathrm{In}_{1-x} \mathrm{Tl}_{x} \mathrm{Sb}$ films. The epilayers, which exhibited a Tl peak in the AES spectrum, showed a clear shift of the X-ray diffraction peak toward a higher angle relative to that of InSb. This implies a decrease of the lattice constant (in the growth direction) of $\mathrm{In}_{1-x} \mathrm{Tl}_{x} \mathrm{Sb}$ compared to the lattice constant of InSb, according to Bragg's law. As $\mathrm{Tl}$ incorporation increases, the diffraction peaks shift 


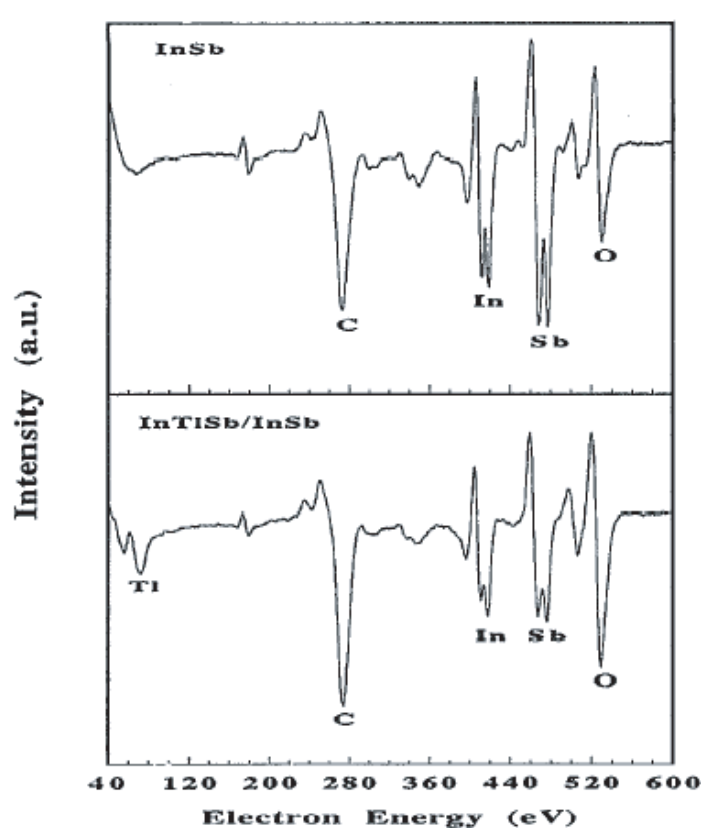

Fig. 5.1. Auger electron spectra of (a) InSb and (b) InTlSb.

gradually toward a higher angle in the experimental range, indicating the gradual decrease of the lattice constants in the growth direction. The lattice constants normal to the growth direction are assessed by asymmetric $\{115\}$ reflections and found to be the same as the lattice constants in the growth direction. These results indicate that the lattice of $\mathrm{In}_{1-x} \mathrm{Tl}_{x} \mathrm{Sb}$ is contracted compared to that of $\mathrm{InSb}$ due to the incorporation of $\mathrm{Tl}$ in InSb. This behavior is particularly striking because it is in direct contrast with the atomic size effect based on tetrahedral covalent radii, which in general holds for isoelectronic impurity incorporation in III antimonides [41]. The low oxidation state of $\mathrm{Tl}$ in $\mathrm{In}_{1-x} \mathrm{Tl}_{x} \mathrm{Sb}$ has been suggested as a possible reason for the observed lattice contraction [42]. The present results indicate that the incorporation behavior of $\mathrm{Tl}$ in $\mathrm{In}_{1-x} \mathrm{Tl}_{x} \mathrm{Sb}$ differs from that of other group III impurities in III antimonides. A curve of the full width at half maximum (FWHM) of the epilayer's (004) diffraction is plotted in Figure 5.3 as a function of $\mathrm{Tl}$ concentration. The trend shows the gradual increase of the FWHM with $\mathrm{Tl}$ incorporation, indicating that the crystallinity of the films is deteriorating.

\subsubsection{Electrical characteristics}

Hall Effect measurement of InTlSb/InSb on semiinsulating GaAs sample was performed and the result is shown in Figure 5.4. The Hall coefficient $R_{H}$ remains negative throughout the entire temperature range and its temperature dependence resembles that of an $n$-type material.

Hall Effect of InTlSb samples with different Tl flow was investigated. As shown in Figure 5.5, room-temperature Hall mobility ranged from $2 \times 10^{4} \mathrm{~cm}^{2} / \mathrm{Vs}$ to $5 \times$ $10^{4} \mathrm{~cm}^{2} / \mathrm{Vs}$, and the electron concentration from $1 \times$

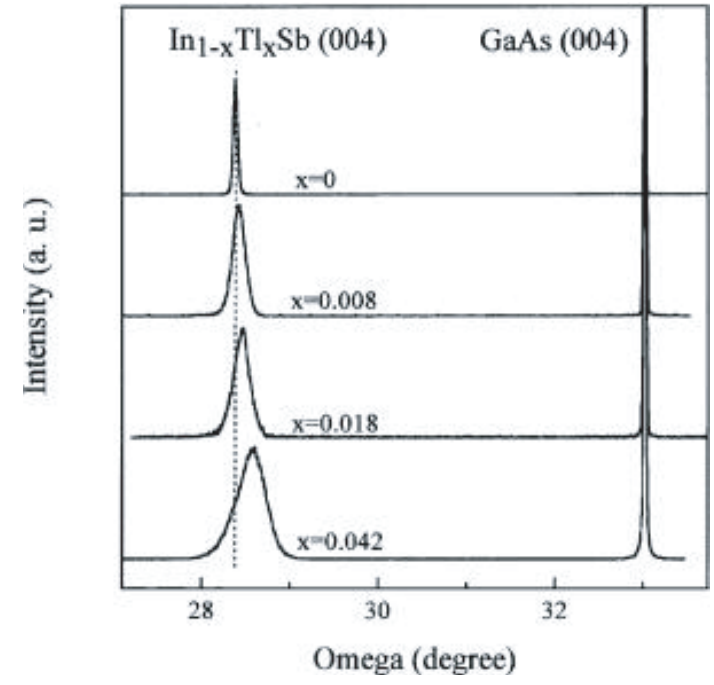

Fig. 5.2. High resolution (004) X-ray diffraction curves of the $\mathrm{In}_{1-x} \mathrm{Tl}_{x} \mathrm{Sb}$ samples grown on the GaAs substrates. The vertical dashed line corresponds to the peak position of the InSb epilayer.

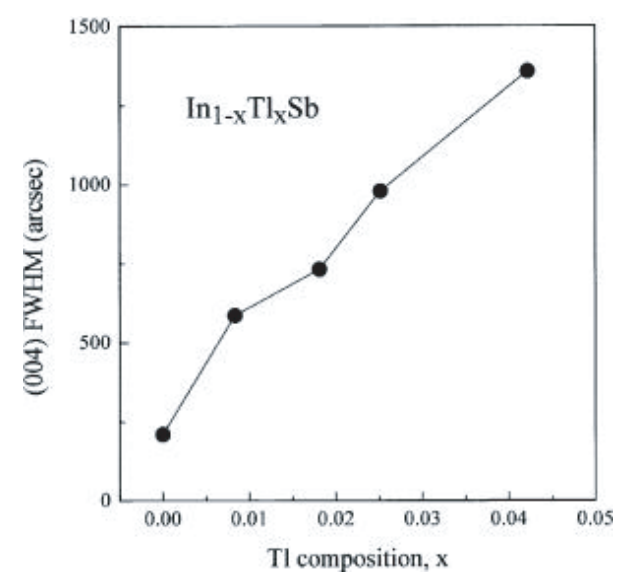

Fig. 5.3. The (004) X-ray diffraction FWHM of $\operatorname{In}_{1-x} \mathrm{Tl}_{x} \mathrm{Sb}$ as a function of $\mathrm{Tl}$ concentration.

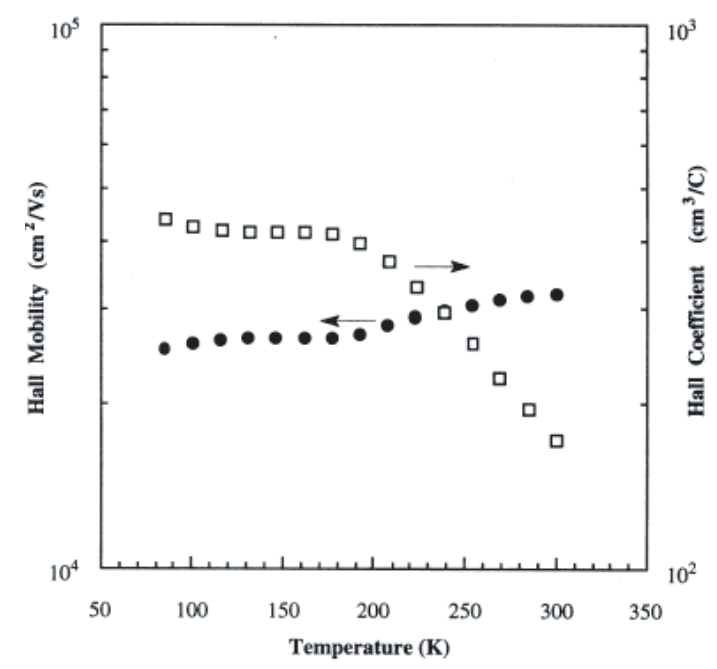

Fig. 5.4. Temperature dependent Hall mobility and coefficient of the InTlSb/InSb film on GaAs. 


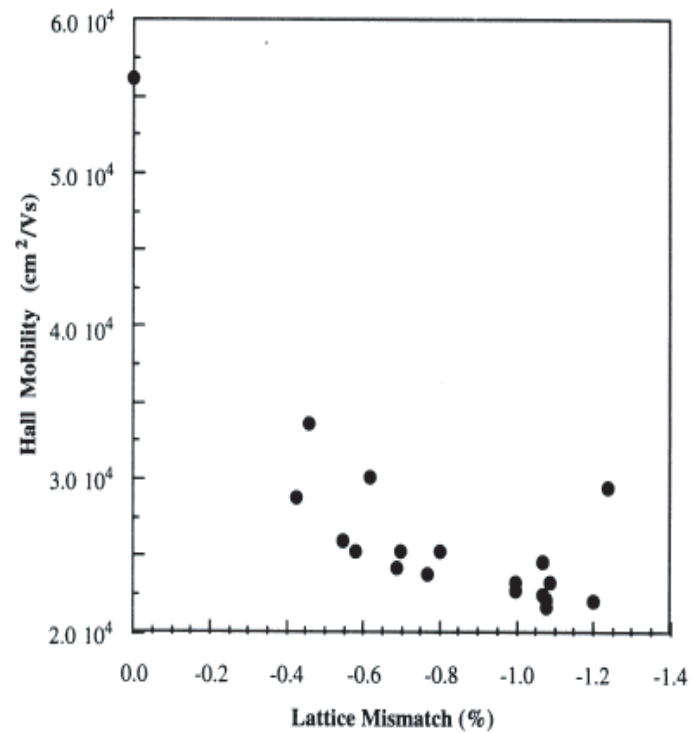

(a)

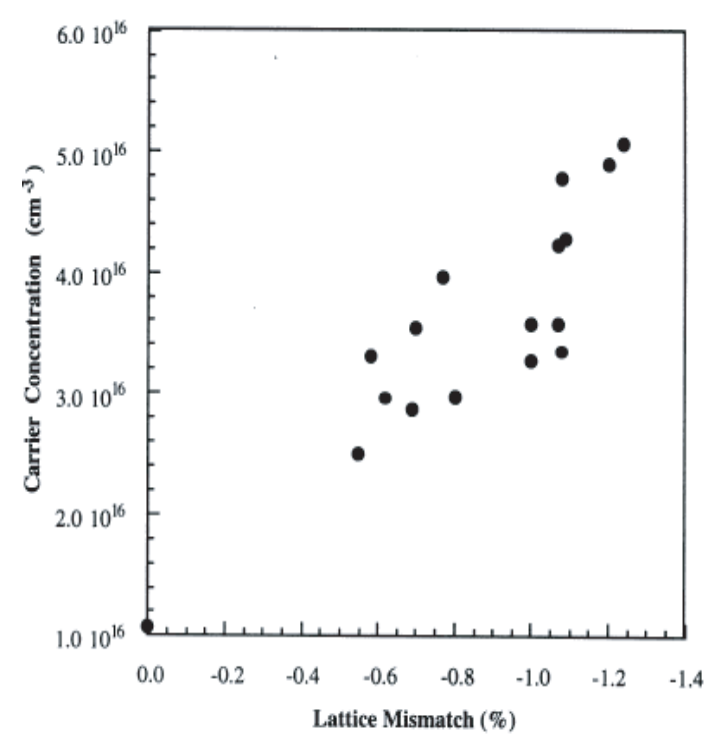

(b)

Fig. 5.5. (a) Dependence of room temperature Hall mobility of InTlSb on the lattice mismatch with InSb. (b) Dependence of room temperature carrier concentration of InTlSb on the lattice mismatch with InSb.

$10^{16} \mathrm{~cm}^{-3}$ to $5 \times 10^{16} \mathrm{~cm}^{-3}$. Since a direct assessment of thallium is difficult, the electrical characteristics have been plotted as a function of the lattice mismatch between InTlSb and InSb which was determined from X-ray diffraction measurements. The lattice mismatch is used here as an indirect indicator of thallium content. The Hall mobility decreased monotonically with increasing thallium flow, while the electron concentration increased simultaneously. The increase in electron concentration is typical of an intrinsic semiconductor with decreasing bandgap. The decrease in mobility is attributed to an increase in alloy scattering with increasing $\mathrm{Tl}$ content. Alloy scattering has been shown to be significant in InSb-based compound such as InAsSb [43-45].

\subsubsection{Optical characteristics}

Figure 5.6 shows the results of the optical transmission measurements of InSb and InTlSb, respectively. The absorption edge of InTlSb is clearly seen beyond that of InSb $\left(\lambda^{-1}=1818 \mathrm{~cm}^{-1}\right)$, verifying a bandgap reduction of InTlSb. However, an accurate assessment of the absorption edge by this method is difficult due to the thin epilayers and the observed Fabry-Perot oscillation. These oscillations are produced by multiple reflections at the airepilayers and epilayers-substrate interfaces, and from the period other optical parameters such as refractive index of the epilayers have been extracted [39].

In order to determine the absorption coefficient, the epilayer has been modeled as a conventional Fabry-Perot etalon filled with an absorbing medium. The reflection and transmission coefficient of each interface were determined using tabulated values for the refractive index of GaAs and InSb, and the refractive index of InTISb was assumed to be the same as that of InSb. The phase shift through the epilayer was determined from the position of the experimental Fabry-Perot extrema. The resulting absorption spectra at $77 \mathrm{~K}$ are given in Figure 5.7. The InSb sample shows a sharp absorption edge at $5.5 \mu \mathrm{m}$. The InTlSb/InSb sample exhibits overall higher absorption, and an absorption tail extending beyond InSb absorption edge is observed. It should be noted that the calculated absorption coefficient for InTlSb/InSb given in the plot corresponds to the average absorption of the two layers weighted by their thickness, respectively.

$$
\alpha=\frac{\alpha_{\mathrm{InTlSb}} d_{\mathrm{InTlSb}}+\alpha_{\mathrm{InSb}} d_{\mathrm{InSb}}}{d_{\mathrm{InTlSb}}+d_{\mathrm{InSb}}}
$$

where $\alpha_{\text {InSb }}$ and $\alpha_{\text {InTISb }}$ are the absorption coefficients of each layer and $d_{\text {InSb }}$ and $d_{\text {InTISb }}$ are their respective thickness. The tendency to attenuate any feature of InTlSb absorption spectrum, and the observed shift should be even more apparent for a thick InTlSb layer.

Figure 5.8 shows the spectral photoresponse from the InSb and InTlSb films grown on GaAs substrates. For the InSb sample, an absorption cutoff wavelength at about $5.5 \mu \mathrm{m}$ was observed at $77 \mathrm{~K}$. The bump around $4.3 \mu \mathrm{m}$ is due to the carbon-dioxide absorption. A distinct shift of absorption edge from $5.5 \mu \mathrm{m}$ to $8.4 \mu \mathrm{m}$ for InTlSb was observed at $77 \mathrm{~K}$. In general agreement with the theoretical prediction, the samples with high $\mathrm{Tl}$ flows give longer response wavelengths. The rough estimation of the $\mathrm{Tl}$ concentration in the samples has been made by linear interpolating the energy band gap between InSb and TlSb from that fact that band gap energy of the grown InTlSb is only slightly deviated from that of InSb. Energy band gap of $-1.5 \mathrm{eV}$ is assumed for the semimetal TlSb. 


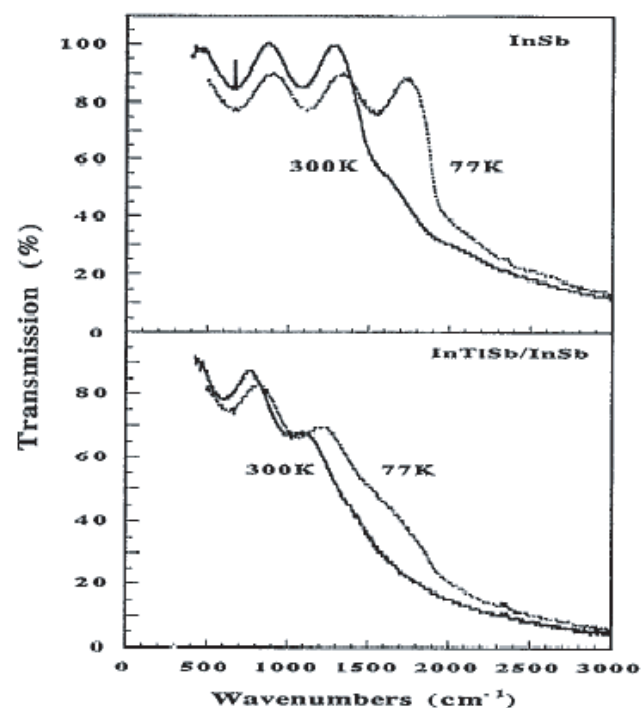

Fig. 5.6. Optical transmission measurements of $\mathrm{InSb}$ and InTlSb at $77 \mathrm{~K}$ and $300 \mathrm{~K}$.

\section{$5.2 \ln$ TISb photodetectors}

In this section we describe the first demonstration of InTlSb photodetectors fabricated on GaAs substrates. Preliminary photodetectors were operated in photoconductive mode. The photoconductors were rectangular shaped with dimension of $3 \times 3 \mathrm{~mm}^{2}$. Ti/Au was evaporated by electron-beam evaporation to make contacts. The resistance is about $50 \Omega$ at room temperature and increases to several thousands $\Omega$ at $77 \mathrm{~K}$. The spectral photoresponse is measured by a FTIR spectrometer with a low-noise preamplifier. The responsivity is calibrated by a blackbody test setup, including a back body source (Mikron 305), preamplifier (EG\&G PA-6), and chopper system. The blackbody temperature is set at $800 \mathrm{~K}$. the modulation frequency is set at $400 \mathrm{~Hz}$ since response measurements as a function of chopper frequency showed that the thermal effect could be neglected at frequencies higher than $200 \mathrm{~Hz}$. The photodetectors were mounted in a liquid nitrogen cooled cryostat system and the measurements were taken at temperatures between $77 \mathrm{~K}$ and $300 \mathrm{~K}$.

The absolute responsivity for an $\mathrm{In}_{0.94} \mathrm{Tl}_{0.06} \mathrm{Sb}$ photodetector is shown in Figure 5.9. The cutoff wavelength extends to $11 \mu \mathrm{m}$ at $300 \mathrm{~K}$. The maximum responsivity in an $\mathrm{In}_{0.96} \mathrm{Tl}_{0.04} \mathrm{Sb}$ photodetector is about $6.64 \mathrm{~V} / \mathrm{W}$ at $77 \mathrm{~K}$. The corresponding Johnson-noise limited detectivity is $7.64 \times 10^{8} \mathrm{~cm} \mathrm{~Hz}^{1 / 2} / \mathrm{W}$. The performance of $\mathrm{In}_{1-x} \mathrm{Tl}_{x} \mathrm{Sb}$ photoconductive detectors are listed in Table 5.2.

\section{$6 \operatorname{lnBiSb}$}

Besides adding As or $\mathrm{Tl}$ to an InSb lattice, it is also possible to substitute Bi. As a result, a rapid reduction of the bandgap is expected. The basis of the prediction is $\mathrm{InBi}$, which has a band gap of $1.5 \mathrm{eV}$. By alloying InBi with $\mathrm{InSb}$, it has been suggested that the band gap energy of

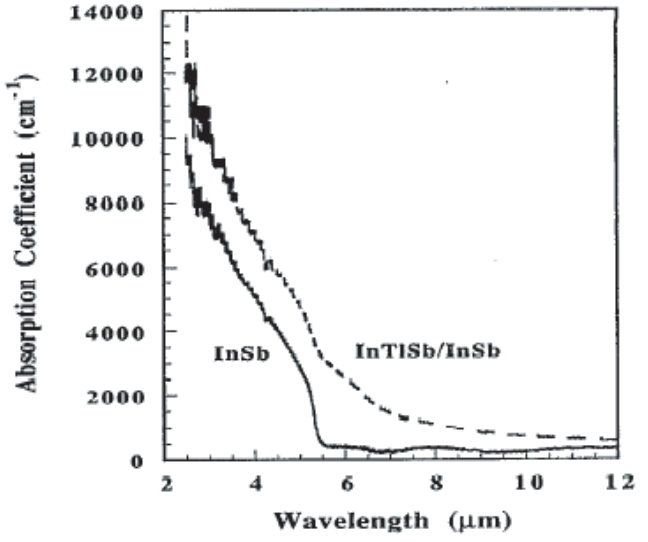

Fig. 5.7. Optical absorption spectra of InSb (solid) and InTISb (dashed) extracted from transmission measurements.

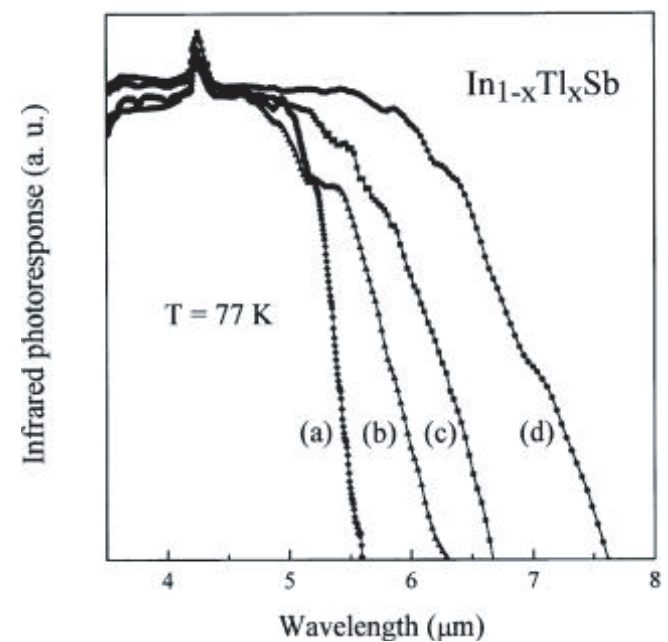

Fig. 5.8. The normalized photoresponse spectra of the $\mathrm{In}_{1-x} \mathrm{Tl}_{x} \mathrm{Sb}$ samples: (a) $x=0$, (b) $x=0.012$, (c) $x=0.021$, and (d) $x=0.034$. The photoconductivity measurements were performed at $77 \mathrm{~K}$.

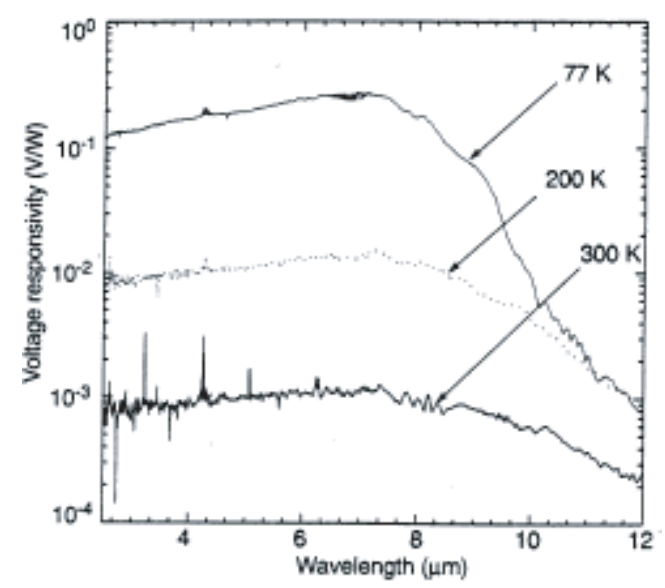

Fig. 5.9. The spectral responsivity of an $\operatorname{In}_{0.9} 4 \mathrm{Tl}_{0.06} \mathrm{Sb}$ photoconductor at 77200 and $300 \mathrm{~K}$. 
Table 5.2. Performance of $\operatorname{In}_{1-x} \mathrm{Tl}_{x} \mathrm{Sb}$ photoconductors at $77 \mathrm{~K}$.

\begin{tabular}{cccccc}
\hline $\operatorname{In}_{1-x} \mathrm{Tl}_{x} \mathrm{Sb}$ & $\begin{array}{c}\lambda_{\text {cut-off }} \\
\mu \mathrm{m}\end{array}$ & $\begin{array}{c}R_{v}^{\mathrm{Max}} \\
(\mathrm{V} / \mathrm{W})\end{array}$ & $\begin{array}{c}D_{\max }^{*} \\
\left(10^{8} \mathrm{~cm} \mathrm{~Hz}^{1 / 2} / \mathrm{W}\right)\end{array}$ & $\begin{array}{c}\mu \\
\left(10^{3} \mathrm{~cm}^{2} / \mathrm{V} \mathrm{s}\right)\end{array}$ & $\begin{array}{c}\tau \\
(\mathrm{ns})\end{array}$ \\
\hline$X=0.023$ & 6.5 & 2.73 & 2.47 & 1.29 & 19.2 \\
$X=0.035$ & 7.3 & 6.64 & 7.64 & 2.00 & 50.1 \\
$X=0.047$ & 8.4 & 3.56 & 8.54 & 2.75 & 40.4 \\
$X=0.053$ & 9.4 & 0.79 & 2.63 & 4.13 & 10.3 \\
\hline
\end{tabular}

Table 6.1. Growth conditions for $\operatorname{InS}_{0.95} \mathrm{Bi}_{0.05}$.

\begin{tabular}{c|c}
\hline Growth temperature & $\sim 456{ }^{\circ} \mathrm{C}$ \\
\hline Growth pressure & 76 torr \\
\hline V/III ratio & $\sim 13$ \\
\hline Growth rate & $\sim 1.3 \mu \mathrm{m} / \mathrm{h}$ \\
\hline Total $\mathrm{H}_{2}$ flow rate & $1500 \mathrm{cc} / \mathrm{min}$ \\
\hline
\end{tabular}

InSbBi could be varied from -1.5 to $0.23 \mathrm{eV}$. Assuming a linear dependence of the band gap energy on the alloy composition, $\mathrm{InSb}_{1-x} \mathrm{Bi}_{x}$ can then be expected to reach a band gap of $0.1 \mathrm{eV}$ at $x=0.08$.

\subsection{MOCVD growth of InSbBi}

For the growth of the InSbBi alloy, InSb buffer was first grown under the optimum growth conditions determined from the InSb investigations. The growth of InSbBi was then attempted by intruding a small flow of TMBi primarily at the optimum growth condition of InSb. Growth conditions such as temperature, pressure, and V/III ratio were varied to obtain good quality films. The highest $\mathrm{Bi}$ concentration in InSbBi was achieved with the growth temperature of $456{ }^{\circ} \mathrm{C}$, growth pressure of 76 torr, and $\mathrm{V} / \mathrm{III}$ ratio of 13 . The growth rate of this layer was about $1.3 \mu \mathrm{m} / \mathrm{h}$.

\subsubsection{Structural characteristics}

Figure 6.1 shows the (004) X-ray diffraction for an as deposited InSbBi layer on InSb substrate. Two distinct peaks around $28.4^{\circ}$ were observed. The X-ray peak corresponding to $\mathrm{InSb}$ substrate is observed at an angle of $28.39^{\circ}$. The X-ray peak at an angle of $28.49^{\circ}$ is attributed to the InSbBi and clearly distinguished from that of $\mathrm{InSb}$ substrate. In order to confirm that the peak at higher angular position corresponds to the InSbBi epilayer, X-ray rocking curves are measured after successive etching of the film. As can bee seen in Figure 6.2, there is no difference in InSb-InSbBi peak angular separation as the intensity of the peak associated with $\mathrm{InSbBi}$, which is proportional to the film thickness, is observed to decrease with respect to that of InSb. This indicates that the X-ray peak at high angular position indeed originates from the $\mathrm{InSbBi}$ epilayer.

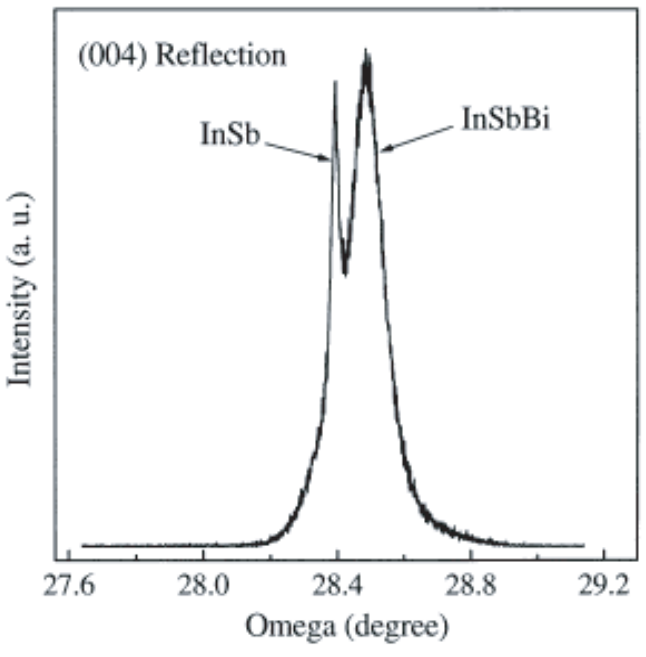

Fig. 6.1. X-ray diffraction of InSbBi/InSb.

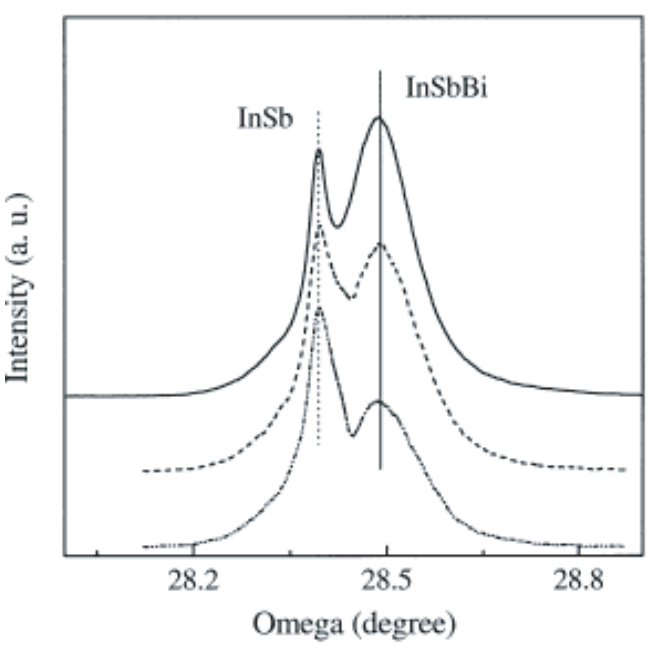

Fig. 6.2. X-ray diffraction profile of the InSbBi epilayer after successive etching.

The study of the dependence of lattice constant on the group $\mathrm{V}$ input partial pressure ratio has been performed. The growth temperature was set at $450{ }^{\circ} \mathrm{C}$ and group $\mathrm{V}$ input partial pressure ratio $\mathrm{Bi} /(\mathrm{Bi}+\mathrm{Sb})$ has been varied from 0.043 to 0.137 . As can be seen in Figure 6.3, quasilinear dependence of lattice constant on group $\mathrm{V}$ input partial pressure ratio was obtained. In order to investigate the crystallinity of the InSbBi thin films, epilayers with different lattice mismatch with InSb have been prepared. 


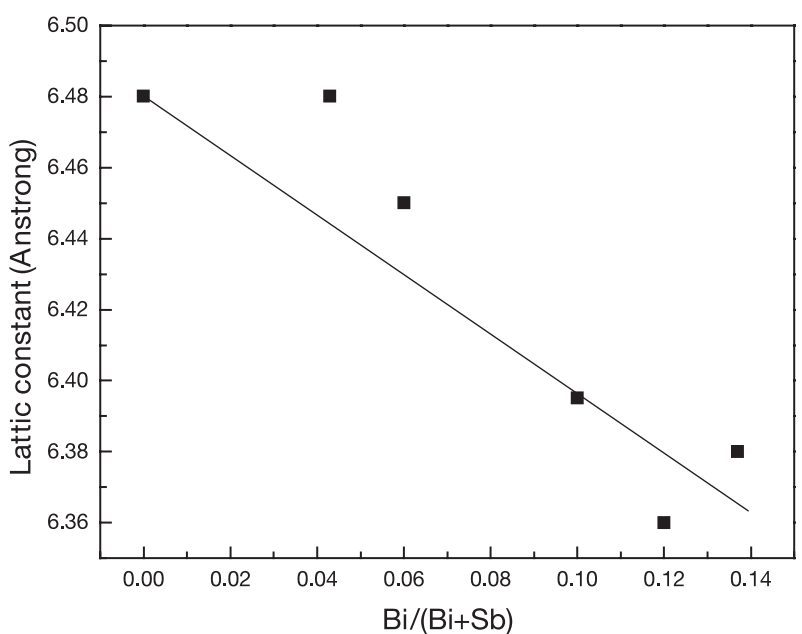

Fig. 6.3. Dependence of lattice constant of the InSbBi epilayer on input $\mathrm{Bi} /(\mathrm{Bi}+\mathrm{Sb})$ ratio.

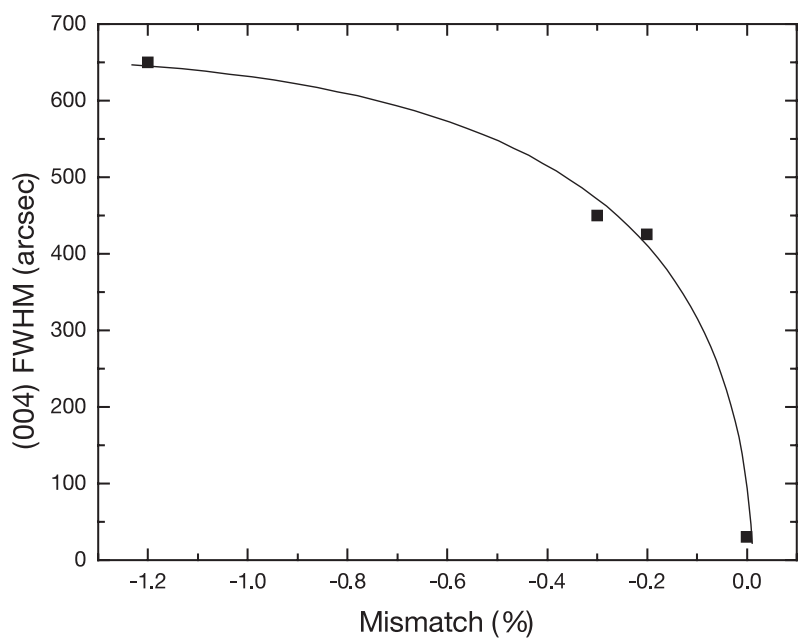

Fig. 6.4. (004) X-ray FWHM of InSbBi on InSb as a function of lattice mismatch with InSb.

Figure 6.4 shows the dependence of X-ray (004) FWHM on the lattice mismatch between InSbBi and InSb. As the mismatch increase, the FWHM increases, indicating worse crystallinity in films with larger lattice mismatch. The broadening of InSbBi peak is attributed to an increase in the defects such as dislocation arising from the increased mismatch with InSb.

\subsubsection{Energy dispersive X-ray analysis}

Energy dispersive X-ray (EDX) spectra for InSb and InS$\mathrm{bBi} / \mathrm{InSb}$ are shown in Figure 6.5. No elements were detected other than $\mathrm{In}, \mathrm{Sb}$, and Bi. Compared to the InSb sample, EDX spectrum of the InSbBi layer clearly showed the presence of a $\mathrm{Bi}$ peak at $2.4 \mathrm{keV}$, which corresponds to a $\mathrm{Bi} \mathrm{M}_{\alpha}$ line. This result further confirms the incorporation of $\mathrm{Bi}$ in the epilayer.
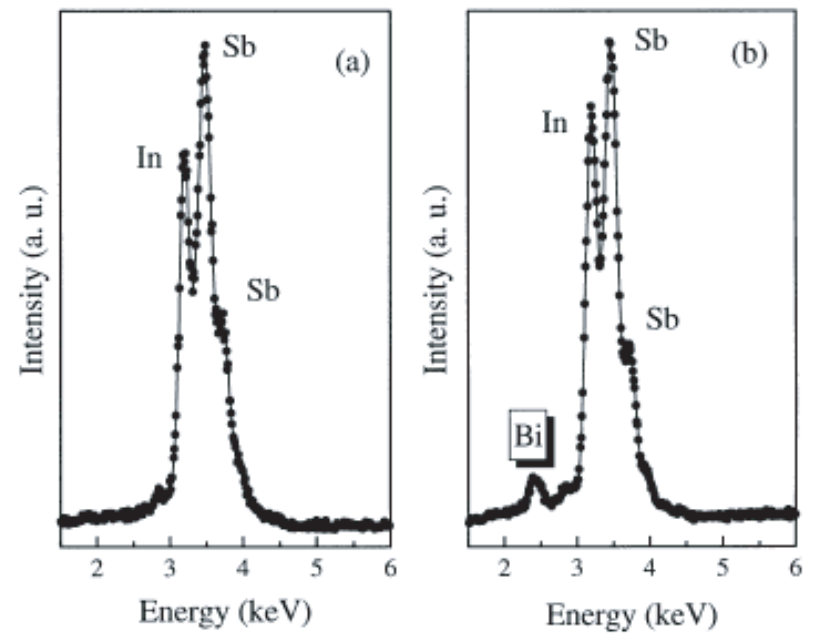

Fig. 6.5. Energy dispersive X-ray analysis spectra of (a) InSb and (b) InSbBi.

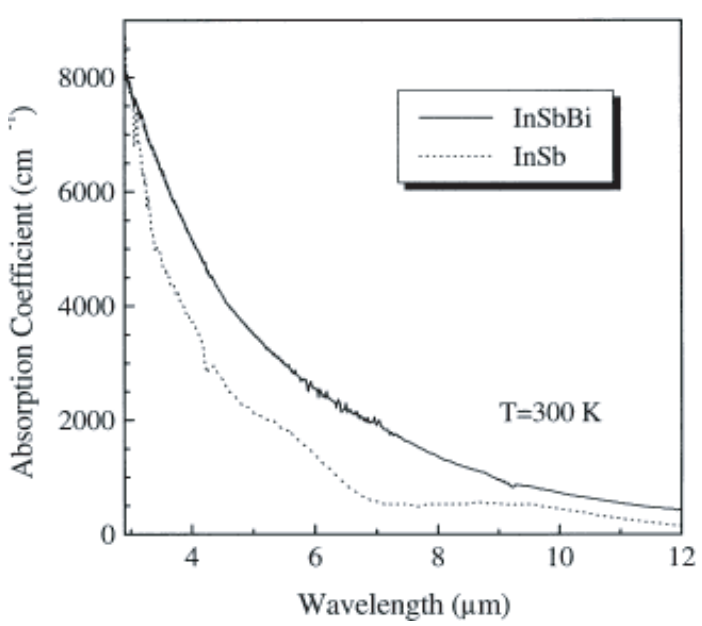

Fig. 6.6. Absorption coefficient of InSbBi at $300 \mathrm{~K}$.

\subsubsection{Optical characteristics}

The reduction of the band gap energy by the addition of $\mathrm{Bi}$ into InSb was verified by optical measurements. Optical absorption measurements were first carried out to study the effect of $\mathrm{Bi}$ incorporation on the band gap energy. Figure 6.6 shows the difference of the absorption coefficients between InSb and InSbBi, which are extracted from the transmission spectra. As can be seen, the difference of the absorption coefficient between the two materials is quite substantial, especially in the range beyond $6 \mu \mathrm{m}$. It is also clear that the incorporation of $\mathrm{Bi}$ in InSb shifts the absorption edge to a smaller energy. The band gap energy of the investigated $\mathrm{InSb}_{0.96} \mathrm{Bi}_{0.04}$ sample roughly estimated from the absorption coefficient of $500 \mathrm{~cm}^{-1}$ was $0.11 \mathrm{eV}$ at room temperature.

Photoresponse measurements were performed using FTIR. Figure 6.7 shows the normalized photoresponse spectra of the selected InSbBi samples along with a photoresponse of a reference InSb. A distinct shift of absorption edge from 5.5 for InSb up to $9.3 \mu \mathrm{m}$ for InSbBi was 


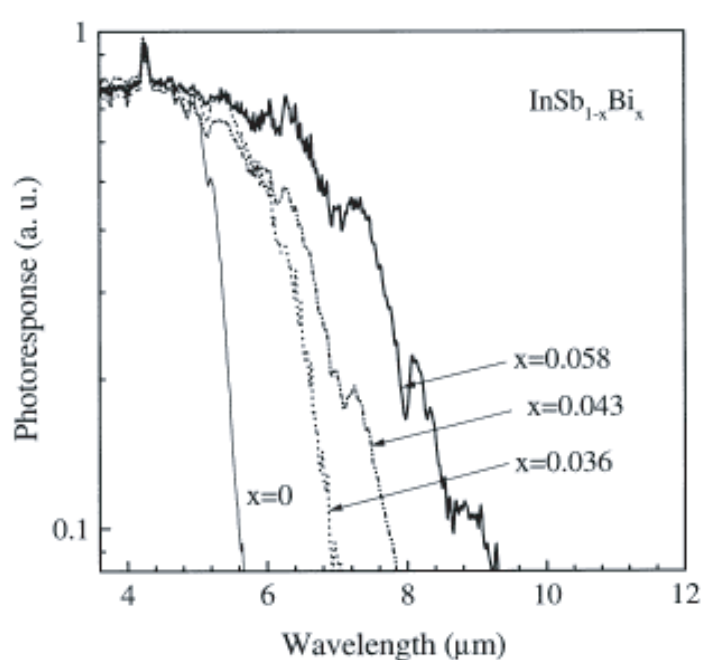

Fig. 6.7. Photoresponse spectra of the InSbBi epilayers with different Bi composition.

observed at $77 \mathrm{~K}$. In general agreement with the theoretical prediction, the samples grown at higher Bi input flows exhibited longer response wavelengths.

The rough estimation of solid Bi concentration in the samples has been made by linearly interpolating the energy band gap between InSb and InBi from the fact that the band gap energy of the grown $\mathrm{InSbBi}$ is deviated only slightly from that of InSb. Energy band gap of $-1.5 \mathrm{eV}$ at $300 \mathrm{~K}$ is assumed for the semimetal InBi. A cutoff wavelength of $9.3 \mu \mathrm{m}$ corresponds to about $5.8 \% \mathrm{Bi}$ incorporation in the $\mathrm{InSbBi}$ layer.

\subsubsection{Electrical characteristics}

The electrical properties of the InSbBi films with thickness varying between $1.4-2.0 \mu \mathrm{m}$ were characterized by Hall measurements. At both $77 \mathrm{~K}$ and $300 \mathrm{~K}, n$-type conductivity has been observed by the incorporation of $\mathrm{Bi}$, which is believed to substitute for $\mathrm{Sb}$ in the InSb lattice.

Figure 6.8 shows the decrease of room temperature mobility from 44100 down to $4910 \mathrm{~cm}^{2} / \mathrm{Vs}$ as the $\mathrm{Bi}$ concentration increase. At $77 \mathrm{~K}$ the mobility decreases from 11000 to $1200 \mathrm{~cm}^{2} / \mathrm{Vs}$ as the Bi concentration increase. The dependence of mobility is probably due to the random alloy scattering potential, which has been shown to be significant in other InSb-based compound such as InAsSb ternary alloy.

As illustrated in Figure 6.9, the electron concentration increased from $3.7 \times 10^{16}$ up to $2.7 \times 10^{17} \mathrm{~cm}^{-3}$ with the increase of $\mathrm{Bi}$ incorporation at $300 \mathrm{~K}$. At $77 \mathrm{~K}$, the electron concentration increase from $4.8 \times 10^{15}$ to $3.9 \times 10^{17} \mathrm{~cm}^{-3}$ as the $\mathrm{Bi}$ concentration increases. The increase of electron concentration with the increase of $\mathrm{Bi}$ concentration is compatible with the decrease of energy band gap in InSbBi ternary alloys.

Temperature dependent Hall measurements were also carried out to examine the Hall mobility and carrier concentration behavior as a function of temperature. Fig-

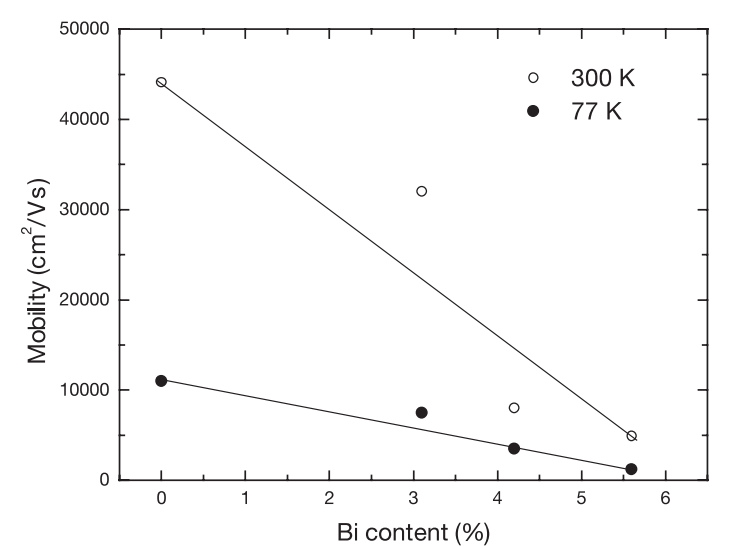

Fig. 6.8. Mobility variation of $\mathrm{InSbBi}$ as a function of Bi content.

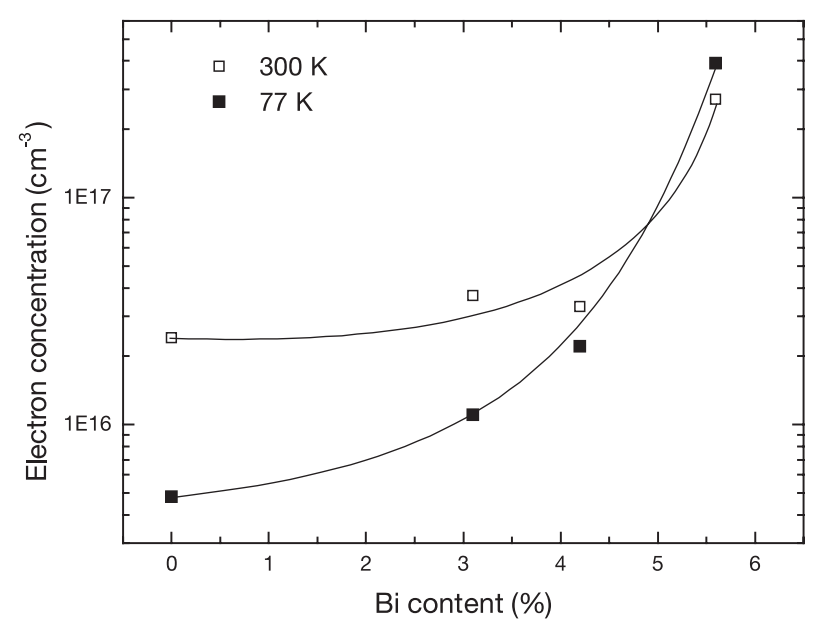

Fig. 6.9. Carrier concentration variation of InSbBi as a function of Bi content.

ure 6.10 shows the mobility as a function of temperature for different $\mathrm{Bi}$ content samples. The mobilities first increase a little bit and then decrease $(x=0,4 \%)$ when temperature decreased from $300 \mathrm{~K}$ to $77 \mathrm{~K}$. The maximum of motility occurs at $250{ }^{\circ} \mathrm{C}$ for $x=0$ and $275{ }^{\circ} \mathrm{C}$ for $x=4 \%$. The decrease in mobility with the decreasing temperature was attributed to the dislocation scattering that dominates at low temperature. Dislocation scattering is expected to be the dominant scattering mechanism at low temperature in a dislocated material with low or moderately doping level.

In Figure 6.11, it is clearly seen that the electron concentration of the $\mathrm{Bi}$ incorporated samples is always larger than that of InSb samples regardless of temperature. The more $\mathrm{Bi}$ is incorporated in to InSb, the less intrinsic behavior is observed in the InSbBi samples. Sample with $5 \%$ Bi content shows almost flat carrier concentration down to $125 \mathrm{~K}$ and then an increase of concentration, implying the unintentional $p$-type doing nature. 


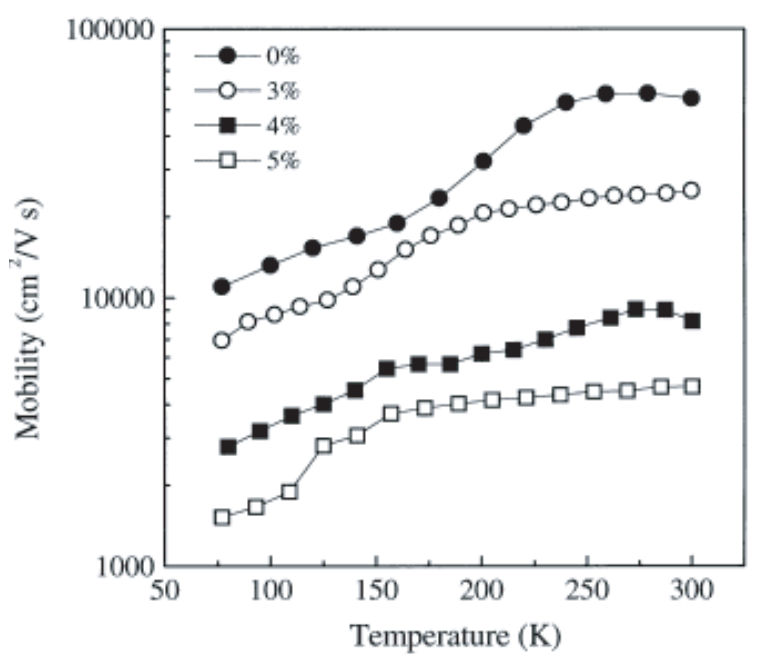

Fig. 6.10. Mobility variation of $\mathrm{InSbBi}$ as a function of temperature.

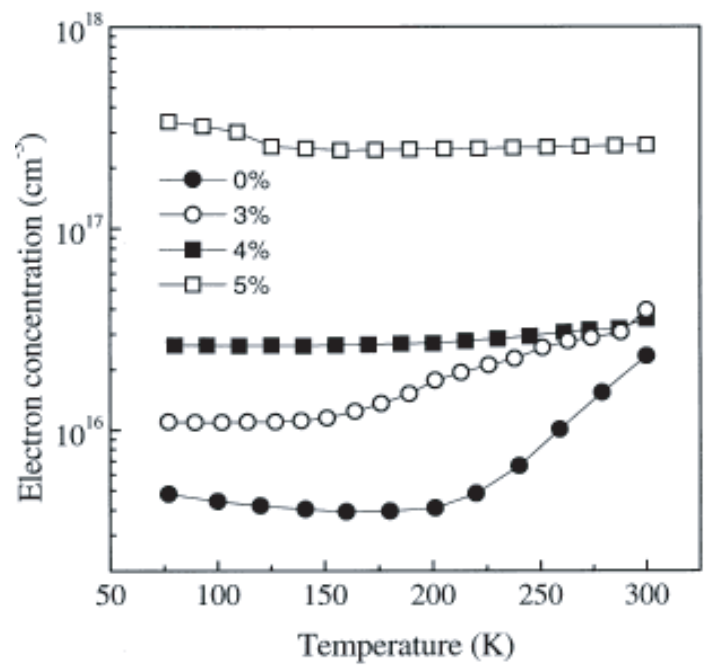

Fig. 6.11. Carrier concentration variation of InSbBi as a function of temperature.

\subsection{InSbBi photodetectors}

The preliminary LWIR InSbBi photoconductors were fabricated from the samples grown on semi-insulating GaAs substrates [46]. $500 \AA \mathrm{Au}$ and $3000 \AA$ Ti were evaporated by electron beam evaporation for the Ohmic contacts. The contact pattern is made using a metal mask, which covers parts of the surface during the evaporation. The active area of the photodetector was $4 \mathrm{~mm}^{2}$ with a $2 \mathrm{~mm}$ electrode spacing. The photodetectors were mounted onto copper heat sinks and the electrical contact was made by an Au-wire bonding. The resistance of an $\mathrm{InSb}_{0.96} \mathrm{Bi}_{0.04}$ photodetector was $435 \Omega$ at $77 \mathrm{~K}$ and $110 \Omega$ at $300 \mathrm{~K}$.

The spectral response at different temperatures of the photodetector fabricated from $\mathrm{InSb}_{0.96} \mathrm{Bi}_{0.04}$ sample is shown in Figure 6.12. The photoresponse cutoff wavelength shifts to $7.7 \mu \mathrm{m}$ at $77 \mathrm{~K}$ and to $9.3 \mu \mathrm{m}$ at $200 \mathrm{~K}$. The responsivity decreases with the increase of tempera-

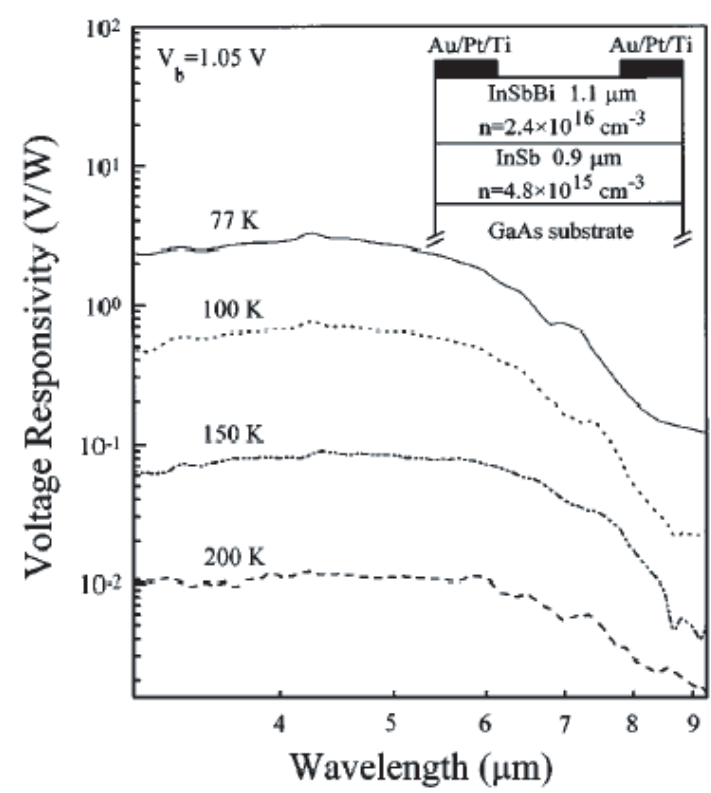

Fig. 6.12. Spectral voltage responsivity of the InSbBi photodetector at different temperatures.

ture. The $\mathrm{S} / \mathrm{N}$ ration was too weak for the adequate measurements at temperatures over $200 \mathrm{~K}$. This decrease of responsivity and $\mathrm{S} / \mathrm{N}$ ratio is due to the increase of intrinsic carriers caused by the decrease of the band gap. The maximum responsivity at $7 \mu \mathrm{m}$ is about $3.2 \mathrm{~V} / \mathrm{W}$. The corresponding Johnson-noise limited detectivity is about $4.7 \times 10^{8} \mathrm{~cm} \mathrm{~Hz}^{1 / 2} / \mathrm{W}$.

The carrier lifetime of the fabricated LWIR detector is estimated from the voltage-dependent responsivity measurements shown in Figure 6.13. The measured responsivity increases linearly with the bias at low bias voltage. However, it can be observed that the responsivity begins to saturate at a bias voltage of $5.5 \mathrm{~V}$ at $77 \mathrm{~K}$. This saturation of responsivity can be explained by the minority carrier sweep-out at the electrode at high bias field. Taking the absorption quantum efficiency as $\eta=0.21$ from infrared transmission measurements, the carrier lifetime mobility obtained from the slope of the linear portion of Figure 6.13 is found to be $\tau \mu=2.35 \times 10^{-4} \mathrm{~cm}^{2} / \mathrm{V}$. So the carrier lifetime is estimated to be about $86 \mathrm{~ns}$ at $77 \mathrm{~K}$.

Room temperature operation photoconductors based on $n$-taped $\mathrm{InSb}_{0.95} \mathrm{Bi}_{0.05}$ have been fabricated and characterized for the first time [47]. Figure 6.14 shows the spectral response of an uncooled $\operatorname{InSb}_{0.95} \mathrm{Bi}_{0.05}$ photoconductor. The cutoff wavelength extends up to $12 \mu \mathrm{m}$ at $300 \mathrm{~K}$. The voltage responsivity at $10.6 \mu \mathrm{m}$ was about $1.9 \mathrm{mV} / \mathrm{W}$ at room temperature and the corresponding Johnson noise limited detectivity was estimated to be about $1.2 \times 10^{6} \mathrm{~cm} \mathrm{~Hz}^{1 / 2} / \mathrm{W}$. The carrier lifetime derived from the voltage dependent responsivity measurements was about $0.7 \mathrm{~ns}$ (Fig. 6.15). 


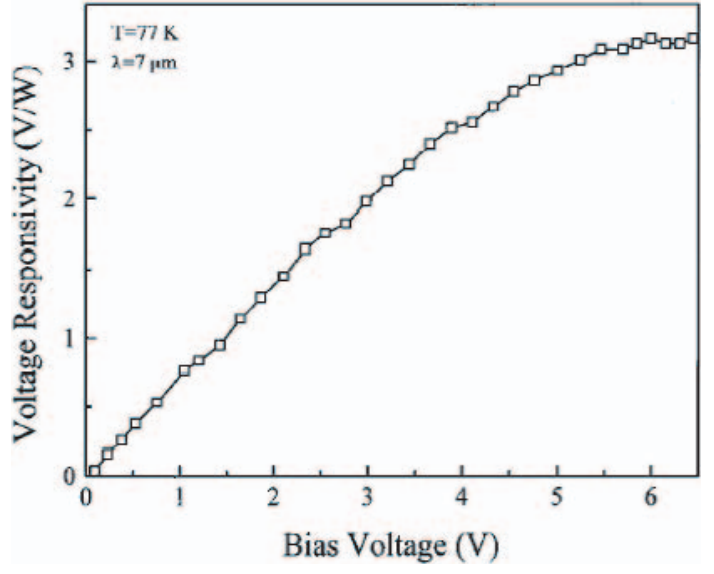

Fig. 6.13. The bias voltage-dependent responsivity of the InSbBi photodetector at $77 \mathrm{~K}$.

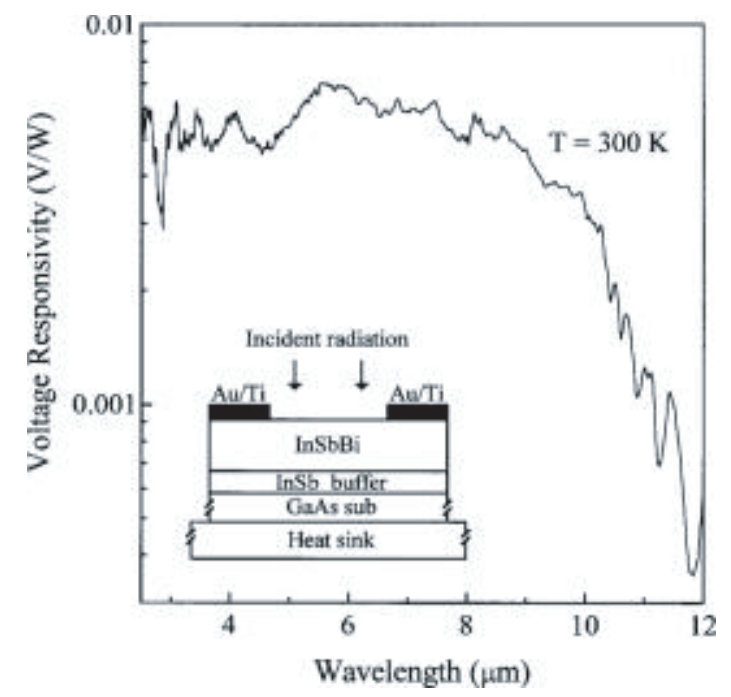

Fig. 6.14. Spectral voltage responsivity of an $\operatorname{InSb}_{0.95} \mathrm{Bi}_{0.05}$ photoconductor at room temperature. The inset shows the schematic of 8-12 $\mu \mathrm{m}$ InSbBi photoconductor.

\section{$7 \ln T I A s S b$}

In order to exceed the limitations imposed by the InTlSb miscibility gap, arsenic was added to form a quaternary material with an extended photoresponse cutoff wavelength. This work lead to first successful demonstration of InTlAsSb on GaAs substrates with a cutoff wavelength up to $15 \mu \mathrm{m}$ at room temperature. These measurements were the first observation of room temperature band edge photo-response at such long wavelengths from III-V alloys.

Film deposition was carried out in a horizontal cold wall LP-MOCVD reactor. TMIn, TMSb, CPTl, and 5\% $\mathrm{AsH}_{3}$ were used as precursors of indium, antimony, thallium, and arsenic, respectively. The reactor pressure was 76 torr for all of the growth and the temperature was around $455{ }^{\circ} \mathrm{C}$. The substrates were superclean semiinsulating GaAs (100).

Figure 7.1 shows the typical X-ray spectra of the InTlAsSb layer grown on the GaAs substrate. There were

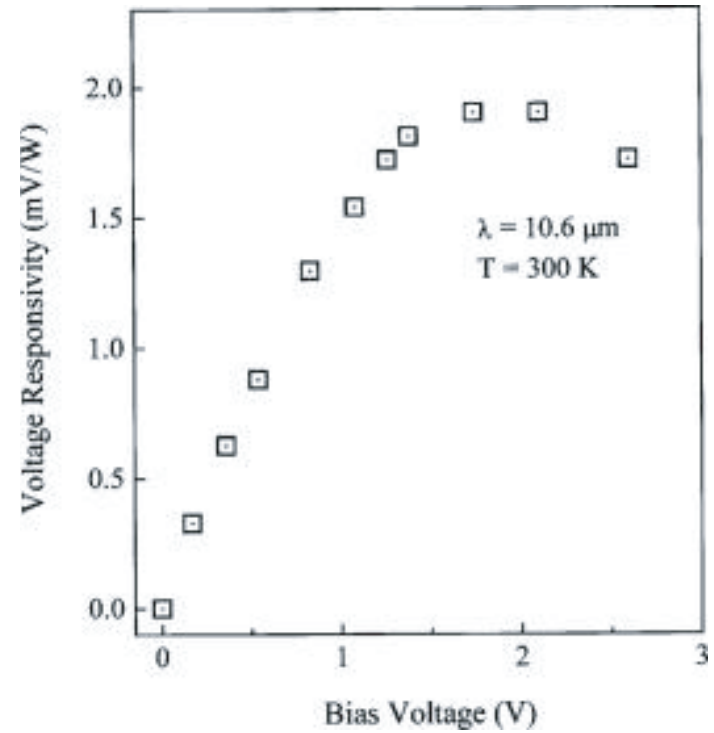

Fig. 6.15. Bias voltage dependent responsivity of an InSb$_{0.95} \mathrm{Bi}_{0.05}$ photoconductor at room temperature.

Table 7.1. Growth parameters for InTlAsSb.

\begin{tabular}{c|c}
\hline Growth pressure & 76 torr \\
\hline Growth temperature & $455{ }^{\circ} \mathrm{C}$ \\
\hline Total $\mathrm{H}_{2}$ flow rate & $1500 \mathrm{cc} / \mathrm{min}$ \\
\hline TMIn flow rate & $3.75 \mu \mathrm{mol} / \mathrm{min}$ \\
\hline $\mathrm{CpTl}$ flow rate & $0.00043 \mu \mathrm{mol} / \mathrm{min}$ \\
\hline $\mathrm{TMSb}$ flow rate & $35.74 \mu \mathrm{mol} / \mathrm{min}$ \\
\hline $\mathrm{AsH}_{3}$ flow rate & $9.38 \mu \mathrm{mol} / \mathrm{min}$ \\
\hline Growth rate & $0.7 \mu / \mathrm{h}$ \\
\hline
\end{tabular}

three peaks at a range between $27^{\circ}$ and $35^{\circ}$. The peaks at $28.4^{\circ}$ and $33.0^{\circ}$ are from the InSb buffer layer and the GaAs substrate, respectively. The remaining peak at $29.1^{\circ}$ was assigned to the (004) diffraction from an InTlAsSb layer. The diffraction spectrum of InTlSb grown with the same In, Tl, and Sb flow rates but only without As was also presented for comparison. It can be clearly seen that the diffraction peak of InTlAsSb is shifted toward a higher angle with respect to that of InTlSb by the As incorporation, confirming the significant incorporation of As into the crystal lattice of InTlSb.

In order to investigate the band gap variation due to the As incorporation into InTlSb, the infrared photoresponse measurements using FTIR was performed. Figure 7.2 shows the normalized spectral photoresponse of InSb, InTlSb, and InTlAsSb grown on SI GaAs substrates. Quaternary sample (b) [(d)] has the same growth conditions with ternary (a) [(c)] except for the As flux. For the InSb sample, an absorption cutoff wavelength at about $5.5 \mu \mathrm{m}$ was observed at $77 \mathrm{~K}$, which agrees with the PL measurement. The feature around $4.3 \mu \mathrm{m}$ is due to the $\mathrm{CO}_{2}$ absorption in the spectrometer. A clear shift of an absorption cutoff wavelength to a longer wavelength up to $8.5 \mu \mathrm{m}$ was obtained for InTlSb, which indicates that 


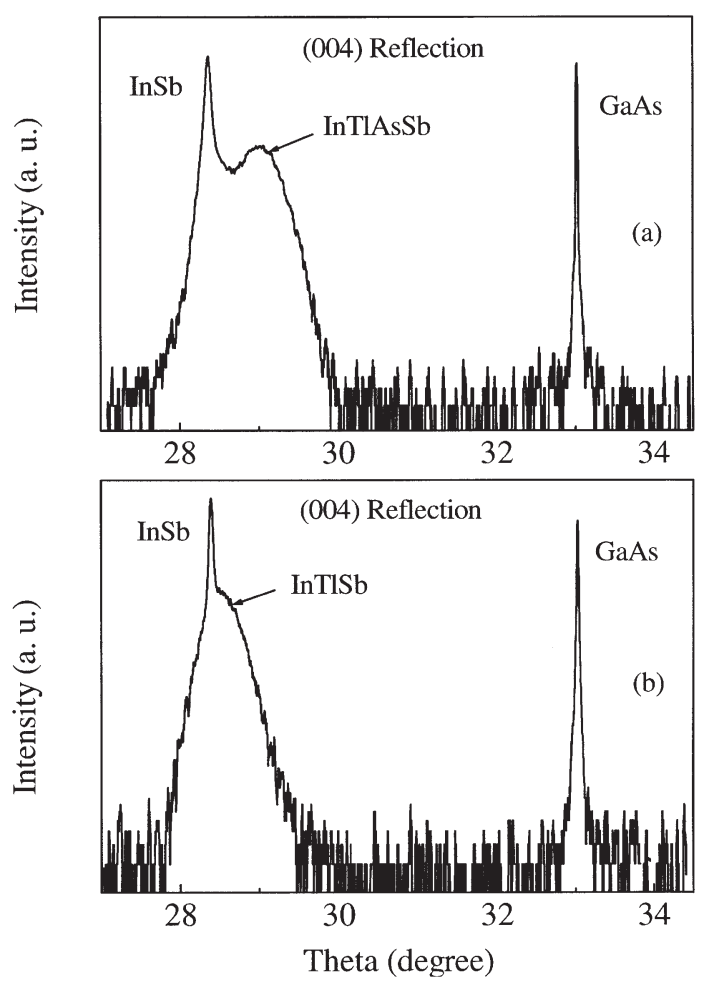

Fig. 7.1. HRXRD patterns of (a) InTlAsSb/InSb/GaAs and (b) InTlSb/InSb/GaAs. The same In, Tl, and $\mathrm{Sb}$ flow rates were used for the growth of InTlAsSb and InTlSb.

decrease of energy gap by $\mathrm{Tl}$ incorporation. Further increase in the cutoff wavelength is achieved by incorporating As into the InTlSb alloy. This indicates that there is a strong bowing effect in InTlAsSb system similar to the case of InAsSb. A cutoff wavelength as long as $10.8 \mu \mathrm{m}$ is obtained from InTlAsSb at $77 \mathrm{~K}$. These results were the first observation of infrared photoresponse at such a long wavelength from the III-V quaternary alloys. The growth conditions for sample (d) are listed in Table 7.1.

The InTlAsSb sample with $10.8 \mu \mathrm{m}$ cutoff wavelength (corresponding to a band gap of $0.115 \mathrm{eV}$ ) exhibited a lattice mismatch of $-0.86 \%$ to InSb. This is in contrast with the InAsSb system that has $0.175 \mathrm{eV}$ band gap energy with the same mismatch to InSb. Note that InAsSb has $-2.27 \%$ lattice mismatch to InSb when the band gap is at minimum $\left(\mathrm{InAs}_{0.65} \mathrm{Sb}_{0.35}: 0.136 \mathrm{eV}\right.$ at $\left.77 \mathrm{~K}\right)$. The present result indicates that InTlAsSb alloy can exhibit longer cutoff wavelength than the InAsSb system while maintaining less lattice mismatch to InSb. Realization of lattice matched InTlAsSb with InSb would improve the device performance significantly by reducing the inevitable generation of misfit dislocation.

Figure 7.3 shows the spectral response of InTlAsSb at room temperature. LWIR photoconductivity up to $15 \mu \mathrm{m}$ was demonstrated for the first time. These measurements were the first observation of room temperature band edge photoresponse at such a long wavelength for the III-V quaternary alloys.

The electrical properties of the InTlAsSb films with thickness of $1.4 \mu \mathrm{m}$ were investigated by Hall measure-

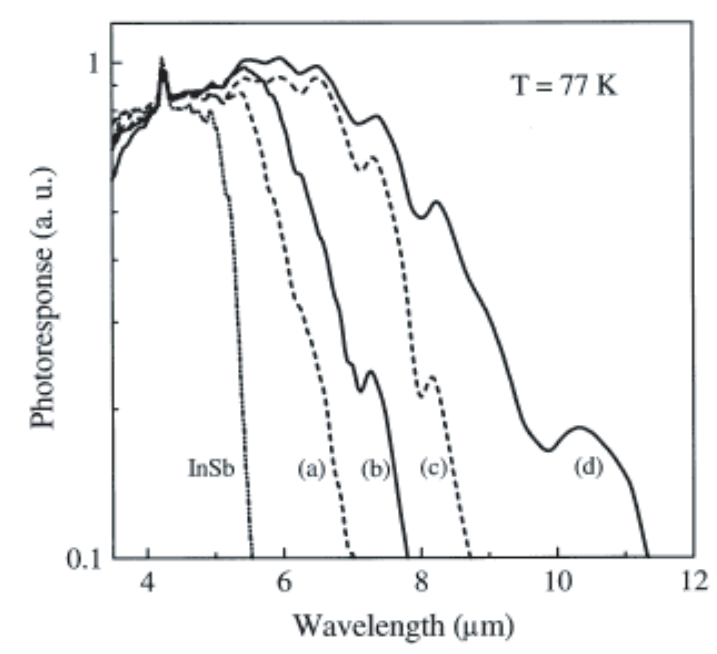

Fig. 7.2. The normalized infrared spectral photoresponse of the InSb, InTiSb (a and c), and InTlAsSb (b and d) films at $77 \mathrm{~K}$. Quaternary sample (b) [(d)] has the identical growth conditions with ternary (a) [(c)] except for the As flow.

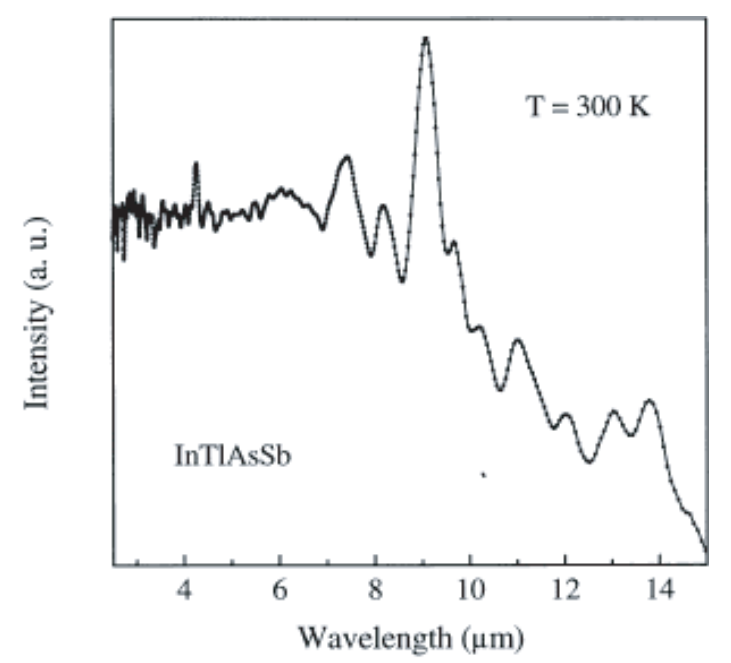

Fig. 7.3. The spectral response of InTlAsSb sample at room temperature.

ments. The Hall coefficients for the undoped samples were negative at room temperature. Figure 7.4 shows the decrease of room temperature mobility from 16100 to $6190 \mathrm{~cm}^{2} / \mathrm{V} \mathrm{s}$ as the flow rate increases. The molar flow rate of TMIn, CpTl, and TMSb were fixed. The decrease of mobility may be due to the increase of impurity and random alloy scattering. With the increase of As flow rate, the $300 \mathrm{~K}$ electron concentration increased from $8.8 \times 10^{16}$ to $3.8 \times 10^{17} \mathrm{~cm}^{-3}$ at $300 \mathrm{~K}$. For the InTlAsSb sample with a $10.8 \mu \mathrm{m}$ cutoff wavelength, the electron concentration was $7.6 \times 10^{16}$ and $1.6 \times 10^{17} \mathrm{~cm}^{-3}$ at $77 \mathrm{~K}$ and $300 \mathrm{~K}$, respectively.

\section{$8 \ln A s S b / \ln A s S b P$ for infrared lasers}

The most thoroughly developed III-V semiconductor for mid-infrared lasers is based on the InAsSb/InAsSbP 


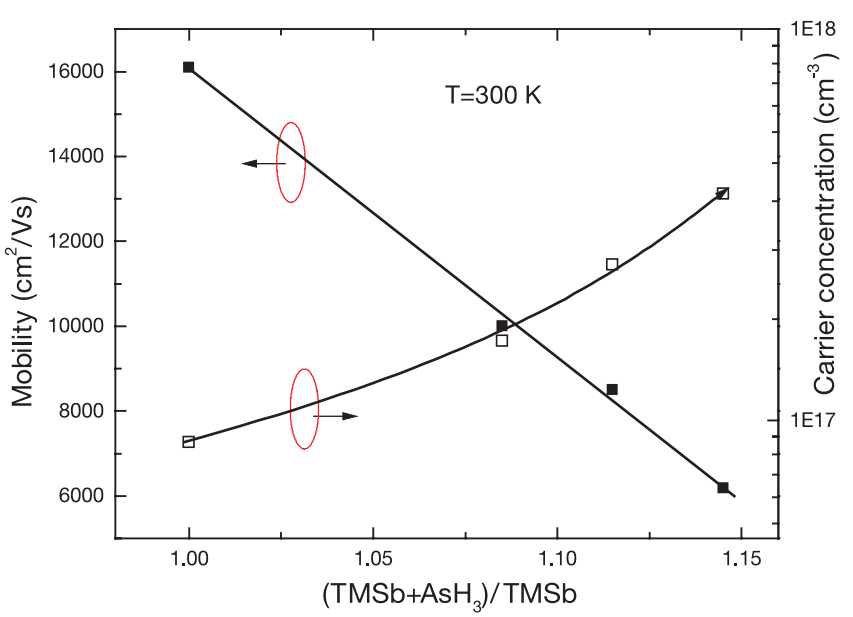

Fig. 7.4. Room temperature mobility and carrier concentration of InTlAsSb as a function of group $\mathrm{V}$ gas flow rate ratio. The molar flow rates of TMIn, $\mathrm{CpTl}$, and $\mathrm{TMSb}$ were fixed.

quaternary alloy grown on InAs substrate. This material system is a promising candidate because it can cover the $3-5 \mu \mathrm{m}$ region by adjusting its alloy composition. Additionally, the InAsSbP material system has a fairly large growth window. The state of the art growth, characterization, and operating characteristics for interband lasers based on the InAsSbP material system will be reviewed and presented throughout this section.

InAsSbP is a quaternary alloy made of InAs, InSb, and InP binary materials. It has a very wide range of energy gap and lattice constant values as can be seen in Figure 8.1 Two binary substrates can be matched with the quaternary alloy: InAs and GaSb. However, InAs substrates were used because they are less expensive and higher quality than GaSb substrates. Furthermore, GaSb has a relatively large refractive index that will reduce the optical confinement of the laser structures.

Fukui and Horikoshi reported the first growth of InAsSbP on InAs substrates by MOCVD in 1980 [49]. They also reported a miscibility gap for compositions with less than $30 \%$ arsenic. Other groups have also demonstrated high quality InAsSbP materials with energy gaps close to theoretical predictions. However, as the arsenic content is reduced, transmission electron micrographs show compositional fluctuations. This has been interpreted as compositions that lie within the miscibility gap. Currently it is accepted that the miscibility gap extends to compositions with less than $20 \%$ arsenic. A schematic of the miscibility gap is shown in Figure 8.2.

\subsection{Growth and characterization of $\ln A s, \ln A s S b$, and InAsSbP}

\subsubsection{Growth and characterization of $\ln A s$}

An Emcore vertical low-pressure MOCVD reactor was used for the growth. The growth of high quality InAs is important since it will serve as the nucleation layer for the entire growth. "Epi-ready" $n^{+}$InAs substrates used

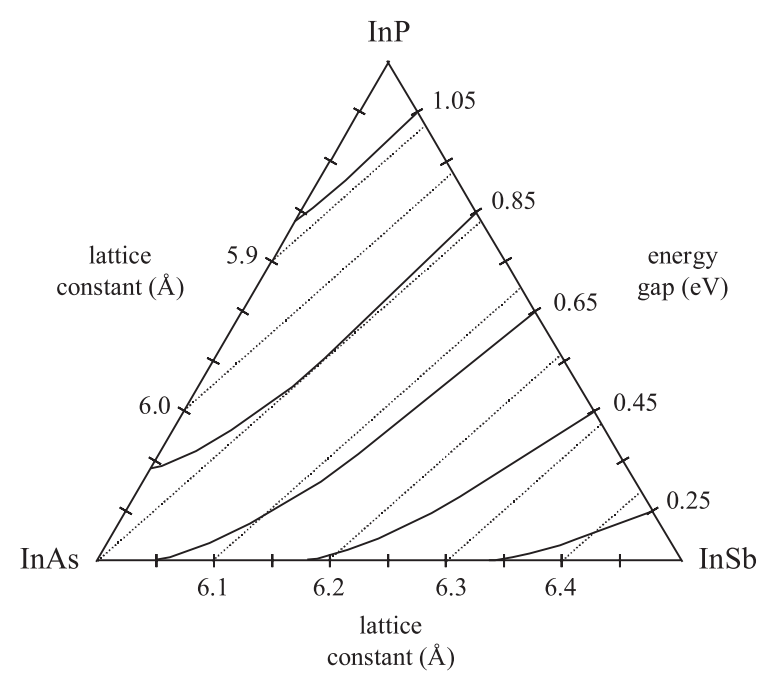

Fig. 8.1. Lattice constant and energy gap values of the InAsSbP material system. Constant lattice constant values are denoted by dotted lines and constant energy gap values are shown with solid lines (after reference [48]).

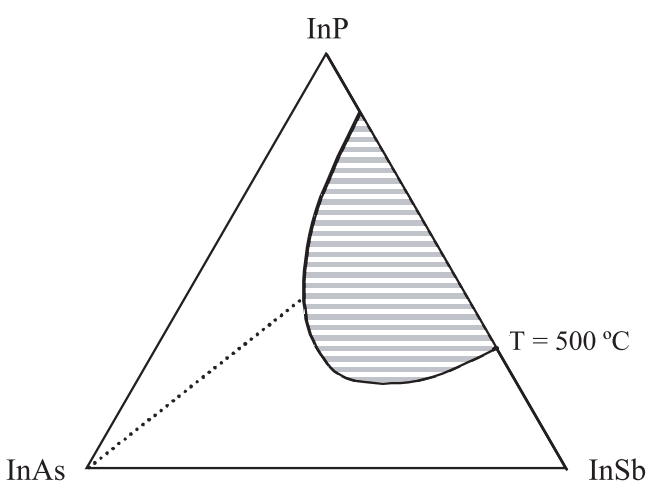

Fig. 8.2. Miscibility gap of the InAsSbP material system. Materials with compositions along the dotted line are lattice matched to InAs [50].

for all growths of InAsSbP based materials. The optimum growth temperature for InAs is approximately $500{ }^{\circ} \mathrm{C}$. The morphology of the optimized InAs is shown in Figure 8.3; the long range scan shows the absence of voids, while the small scan range shows atomic steps resulting from the $2^{\circ}$ miscut of the substrate. The long range surface roughness is similar to that of the substrate prior to growth: $0.79 \AA$ versus $0.61 \AA$.

\subsubsection{Growth and characterization of $\ln \mathrm{AsSb}$}

In order to optimize the growth temperature, temperatures between 500 and $680{ }^{\circ} \mathrm{C}$ were studied for $\mathrm{InAs}_{0.95} \mathrm{Sb}_{0.05}$. The TMIn flow rates were kept constant at $185 \mathrm{ccm}$ or $\mathrm{cm}^{3} / \mathrm{min}$. The material characterization is shown in Figure 8.4.

From Figure 8.4, it is apparent that the material characterization is optimized at roughly the same temperature. Specifically, the PL FWHM is minimum, the X-ray 
Table 8.1. Physical properties of InAs, InSb, and InP [51].

\begin{tabular}{|c|c|c|c|c|}
\hline & $T(\mathrm{~K})$ & InAs & $\mathrm{InSb}$ & $\mathrm{InP}$ \\
\hline Crystal structure & & Cub $(\mathrm{ZnS})$ & Cub $(\mathrm{ZnS})$ & $\mathrm{Cub}(\mathrm{ZnS})$ \\
\hline Lattice constant $(\AA)$ & 300 & 6.0584 & 6.4788 & 5.8688 \\
\hline \multirow[t]{2}{*}{ Energy gap $(\mathrm{eV})$} & 300 & 0.36 & 0.18 & 1.35 \\
\hline & 0 & 0.41 & 0.36 & 1.42 \\
\hline Melting point $(\mathrm{K})$ & & 1215 & 803 & 1335 \\
\hline $\begin{array}{l}\text { Coef. of thermal expansion } \\
\left(10^{-6} \mathrm{~K}^{-1}\right)\end{array}$ & 300 & 4.52 & 5.04 & 5.0 \\
\hline$m_{e}^{*} / m_{0}$ & 4 & 0.024 & 0.014 & 0.077 \\
\hline$m_{l h}^{*} / m_{0}$ & 4 & 0.025 & 0.018 & 0.12 \\
\hline$m_{h h}^{*} / m_{0}$ & 4 & 0.37 & 0.4 & 0.55 \\
\hline$m_{\text {sos }}^{*} / m_{0}$ & 4 & 0.14 & 0.44 & 0.12 \\
\hline \multirow[t]{2}{*}{ Electron mobility $\left(\mathrm{cm}^{2} / \mathrm{V} \mathrm{s}\right)$} & 300 & $3 \times 10^{4}$ & $8 \times 10^{4}$ & \\
\hline & 77 & $8 \times 10^{4}$ & $10^{6}$ & $4 \times 10^{4}$ \\
\hline \multirow[t]{2}{*}{ Hole mobility $\left(\mathrm{cm}^{2} / \mathrm{Vs}\right)$} & 300 & 200 & 850 & 150 \\
\hline & 77 & 500 & $10^{4}$ & 1200 \\
\hline Intrinsic carrier conc. $\left(\mathrm{cm}^{-3}\right)$ & 300 & $9 \times 10^{14}$ & $2 \times 10^{16}$ & $1 \times 10^{8}$ \\
\hline Static dielectric constant & 15.15 & 16.8 & 12.5 & \\
\hline $\begin{array}{l}\text { High frequency dielectric } \\
\text { constant }\end{array}$ & & 12.25 & 15.68 & 9.61 \\
\hline Elastic moduli $\left(10^{12}\right.$ dyn $\left.\mathrm{cm}^{2}\right)$ & 300 & & & \\
\hline $\mathrm{C}_{11}$ & & 8.329 & 6.669 & 10.11 \\
\hline $\mathrm{C}_{12}$ & & 4.526 & 3.645 & 6.61 \\
\hline $\mathrm{C}_{44}$ & & 3.959 & 3.626 & 4.56 \\
\hline Refractive index & & 3.44 & 3.96 & 3.35 \\
\hline \multicolumn{5}{|l|}{ Optical phonon $\left(\mathrm{cm}^{-1}\right)$} \\
\hline $\mathrm{LO}$ & & 242 & 193 & 320 \\
\hline TO & & 220 & 185 & 288 \\
\hline
\end{tabular}
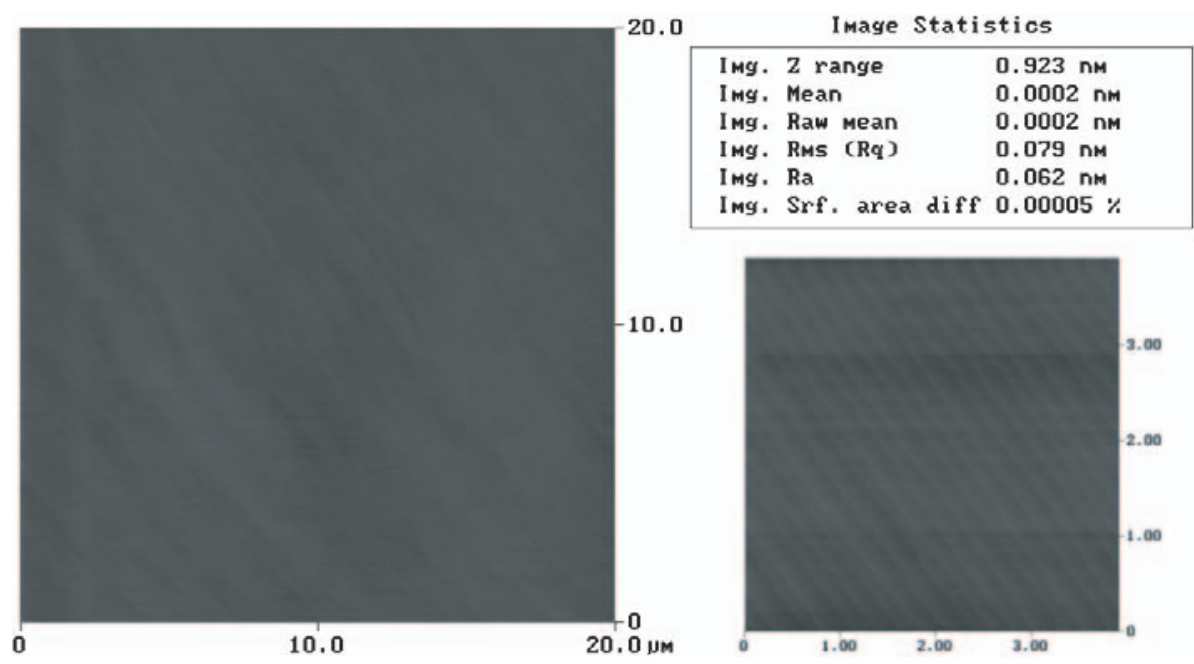

Fig. 8.3. Morphology of optimized homo-epitaxial InAs. The image statistics are shown for the long range scan $(20 \mu \mathrm{m} \times 20 \mu \mathrm{m})$. 


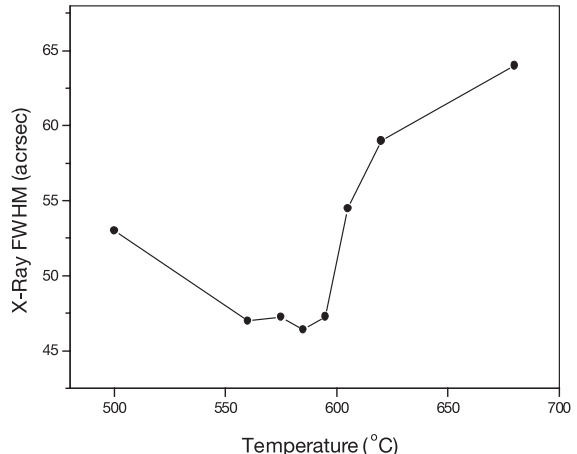

a)

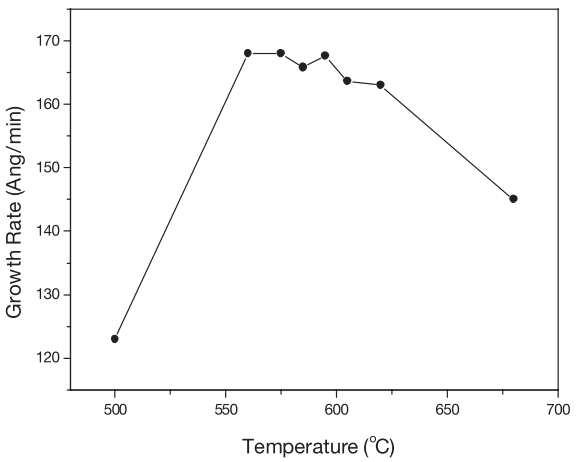

c)

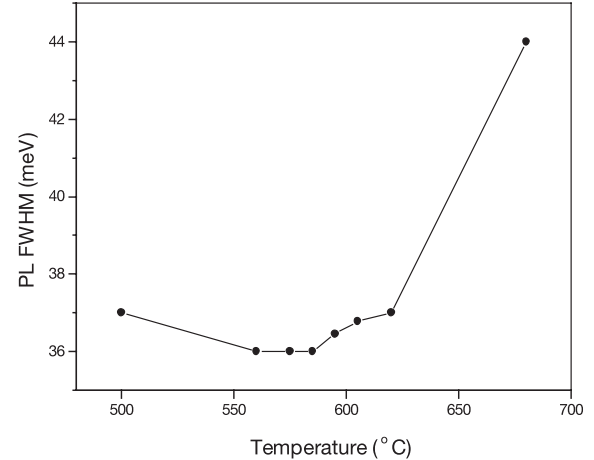

b)

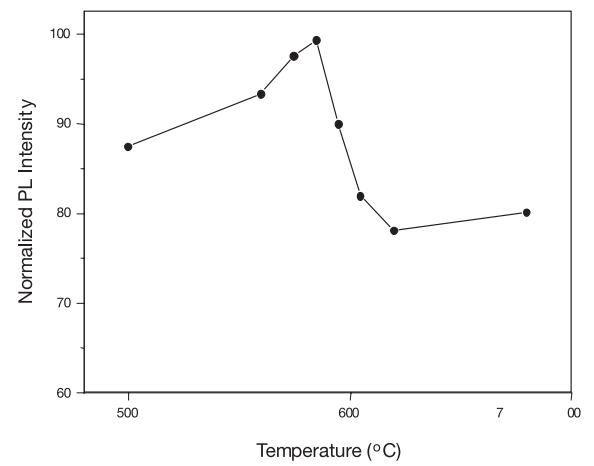

d)

Fig. 8.4. Material characterization of InAsSb versus different growth temperatures. (a) X-ray FWHM, (b) PL FWHM, (c) growth rate, and (d) relative PL intensity.

FWHM is minimum, and the PL intensity is maximum at $585{ }^{\circ} \mathrm{C}$. Therefore, both the optical and structural quality of the layers is optimized at the same temperature of $585^{\circ} \mathrm{C}$. Secondly, the growth rate is not a strong function of temperature between 560 and $605{ }^{\circ} \mathrm{C}$. Furthermore, the surface morphology of these samples is mirror like with no defects visible under $100 \times$ magnification.

The X-ray FWHM was used to optimize the growth rate. A growth temperature of $585^{\circ} \mathrm{C}$ was used and the flow rates of $\mathrm{AsH}_{3}$ and $\mathrm{TMSb}$ were kept constant for each 30 minute growth. It is seen from Figure 8.5 the X-ray FWHM decreases monotonically with decreasing growth rate with a sharp increase in the FWHM after a growth rate of $180 \AA / \mathrm{min}$. However, there are negative effects associated with a longer growth time. First, the inherent reactor temperature drift will occur for longer period of time causing small changes in the material composition. Second, the background carrier concentration increases with the growth time. For these reasons, growth rates of approximately $150-170 \AA /$ min were used.

The optimized growth conditions determined by the procedure above resulted in high quality InAsSb. The $\mathrm{X}$-ray diffraction spectra is shown in Figure 8.6. Its high structural quality is supported by the narrow FWHM of 45 arcs and interference (Pendellosung) fringes which correspond to the epilayer thickness. The morphology is mirrorlike with no defects resulting from growth. The surface topology has been measured with AFM and the long range RMS surface roughness is approximately one monolayer. The atomic steps can be seen with AFM and are shown in

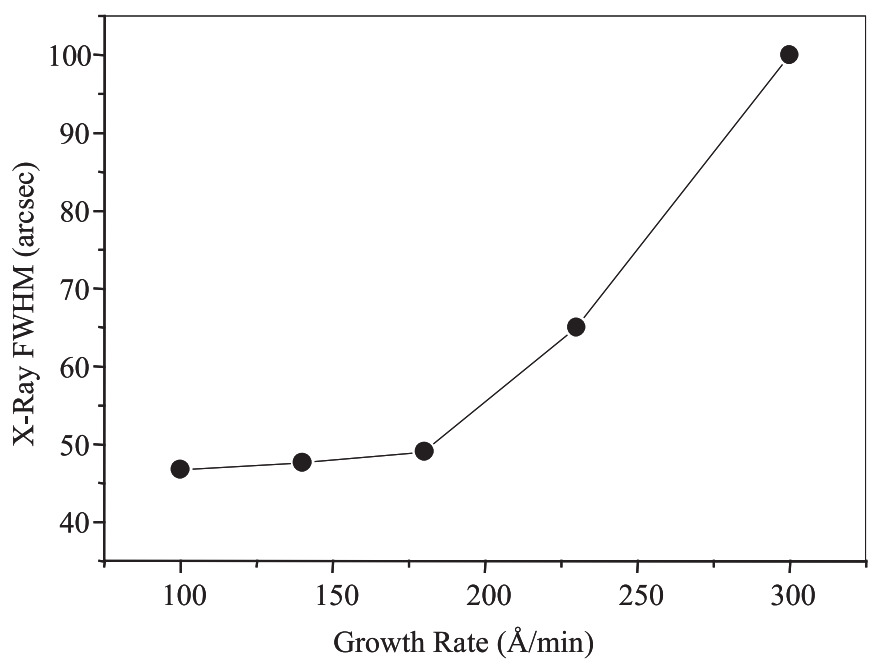

Fig. 8.5. X-ray FWHM versus InAsSb growth rate.

Figure 8.7. The optical quality has been verified with photoluminescence. Again the FWHM at low temperatures is very low at $25 \mathrm{meV}$. The PL spectra are shown in Figures 8.8 and 8.9 .

\subsubsection{Growth and characterization of $\ln \mathrm{AsSbP}$}

The InAsSbP layers will be the cladding layers; their function is to provide electronic and optical confinement. It is 


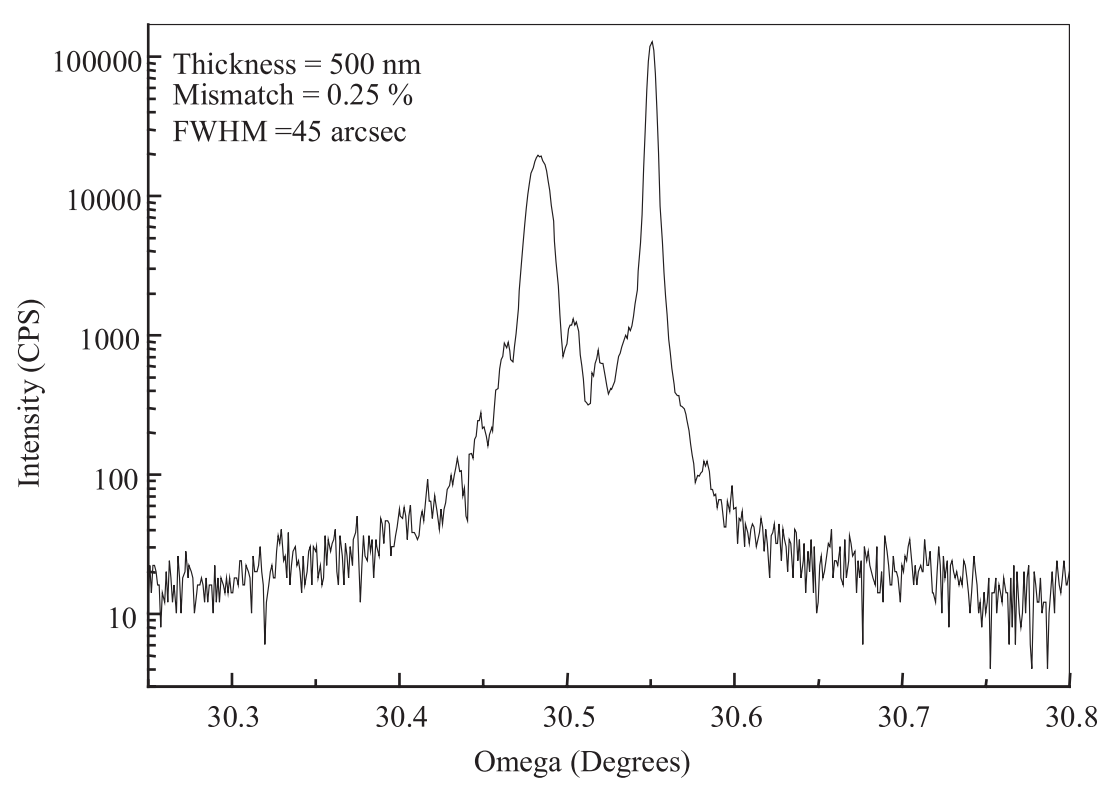

Fig. 8.6. X-ray of a $500 \mathrm{~nm}$ thick InAsSb epilayer.

most important that the cladding layer be lattice matched to the active region to avoid misfit dislocations that may act as non-radiative recombination centers. From this information, the optimal cladding layer composition is $\operatorname{InAs}_{0.20} \mathrm{Sb}_{0.30} \mathrm{P}_{0.50}$.

The percent pyrolysis and distribution coefficients for the three group V materials are the main physical parameters that determine the final material composition. The distribution coefficient can be thought of as the ratio of the amount of source that incorporates into the crystal to the amount of source that contacts the substrate. Furthermore, these parameters are all a function of temperature and so the material composition versus growth temperature relationship is important to understand. For InAsSb, as the temperature increases, the pyrolysis of $\mathrm{AsH}_{3}$ increases while the distribution coefficient of $\mathrm{Sb}$ decreases sharply. Therefore, holding all other variables constant, as the temperature increases the amount of $\mathrm{Sb}$ in the epilayer decreases. For InAsSbP, only the understanding of $\mathrm{P}$ needs to be added; the same percentage pyrolysis of $\mathrm{PH}_{3}$ and $\mathrm{AsH}_{3}$ occurs about at approximately $100{ }^{\circ} \mathrm{C}$ higher for $\mathrm{PH}_{3}$. So only increasing the growth temperature will result in less $\mathrm{Sb}$ and more $\mathrm{P}$. This hypothesis is verified experimentally and the results are shown in Figure 8.10. Using photoluminescence and X-ray characterization techniques, the composition was determined. This is shown in Figure 8.11. As the growth temperature increases from 561 to $601{ }^{\circ} \mathrm{C}$, the $\mathrm{P}$ composition increases from $0.52 \%$ to $0.56 \%$, or about $\pm 0.01 \%$ per $\pm 10{ }^{\circ} \mathrm{C}$. Within experimental error, this relationship is linear. This confirms that, although there is inherently some temperature drift during the growth, there will not be a detectable change in material composition resulting from this temperature drift.

Another important relationship is that between the $\mathrm{AsH}_{3}$ flow rate and composition. This can be explained by understanding the nature of the pyrolysis of $\mathrm{AsH}_{3}, \mathrm{TMSb}$, and $\mathrm{PH}_{3}$ as shown in the following equations:

$$
\begin{aligned}
\mathrm{AsH}_{3} & \longleftrightarrow \frac{1}{4} \mathrm{As}_{4}+3 \mathrm{H} \\
\mathrm{PH}_{3} & \longleftrightarrow \frac{1}{4} \mathrm{P}_{4}+3 \mathrm{H} \\
\mathrm{Sb}\left(\mathrm{CH}_{3}\right)_{3}+3 \mathrm{H} & \longleftrightarrow \mathrm{Sb}+3 \mathrm{CH}_{4}
\end{aligned}
$$

An important result of the pyrolysis of $\mathrm{AsH}_{3}$ is the byproduct of $\mathrm{H}_{2}$. Also, the pyrolysis of TMSb requires a hydrogen rich environment. Therefore, as the amount of $\mathrm{AsH}_{3}$ is increased, the amount of hydrogen increases thus facilitating TMSb decomposition. This relationship has been experimentally confirmed and is shown in Figure 8.12.

Finally, the composition of InAsSbP, within the range of compositions used in this study, can easily be controlled via the flow rates of the sources. Figure 8.13 corroborates this conclusion using $\mathrm{PH}_{3}$ as the source.

The optimized growth conditions resulted in high quality $\mathrm{InAs}_{0.20} \mathrm{Sb}_{0.30} \mathrm{P}_{0.50}$. A typical X-ray diffraction spectra is shown in Figure 8.14. The morphology is mirrorlike. The surface topology has been measured with AFM and the RMS surface roughness is approximately the same as InAsSb or about one monolayer. A typical AFM scan is shown in Figure 8.15. The optical quality and composition has been verified with photoluminescence (Fig. 8.16). As a result of the long diffusion length for photo-excited carriers and the lower energy gap of the InAs substrate, many of the carriers diffuse and recombine in the InAs layer. Therefore, the InAsSbP epilayer and the InAs substrate have similar emission intensities.

\subsubsection{Doping}

TeSn is used as $n$-type dopant. The bubbler pressure and temperature for TeSn were 700 torr and $-10{ }^{\circ} \mathrm{C}$. 


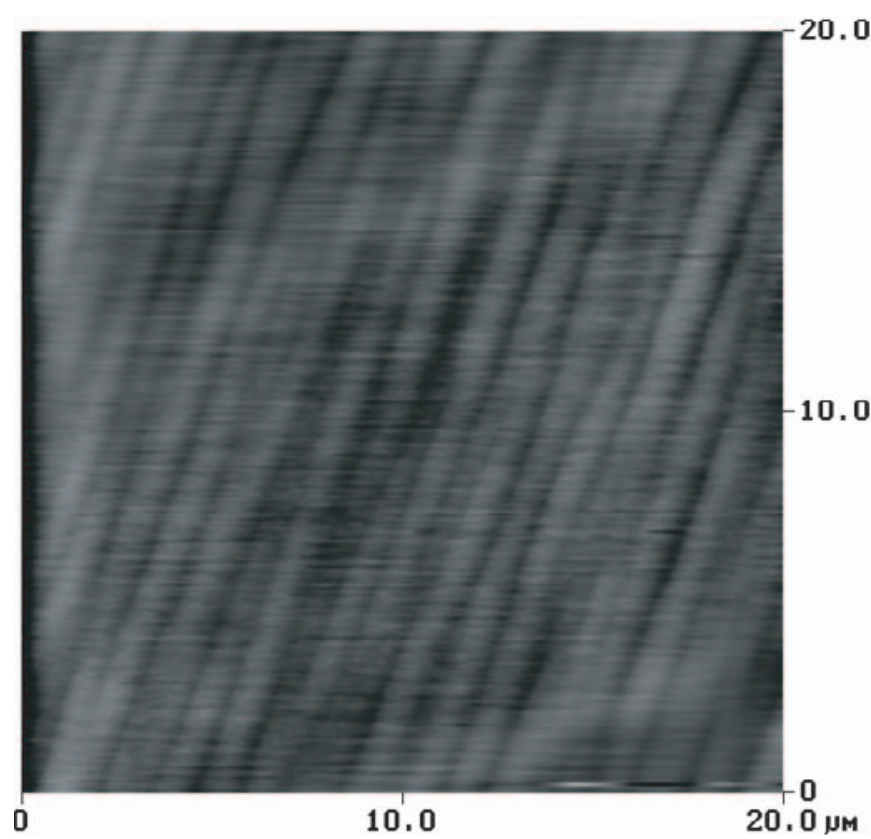

Fig. 8.7. AFM of $500 \mathrm{~nm}$ thick InAsSb showing atomic steps. The height scale is $10 \mathrm{~nm}$.

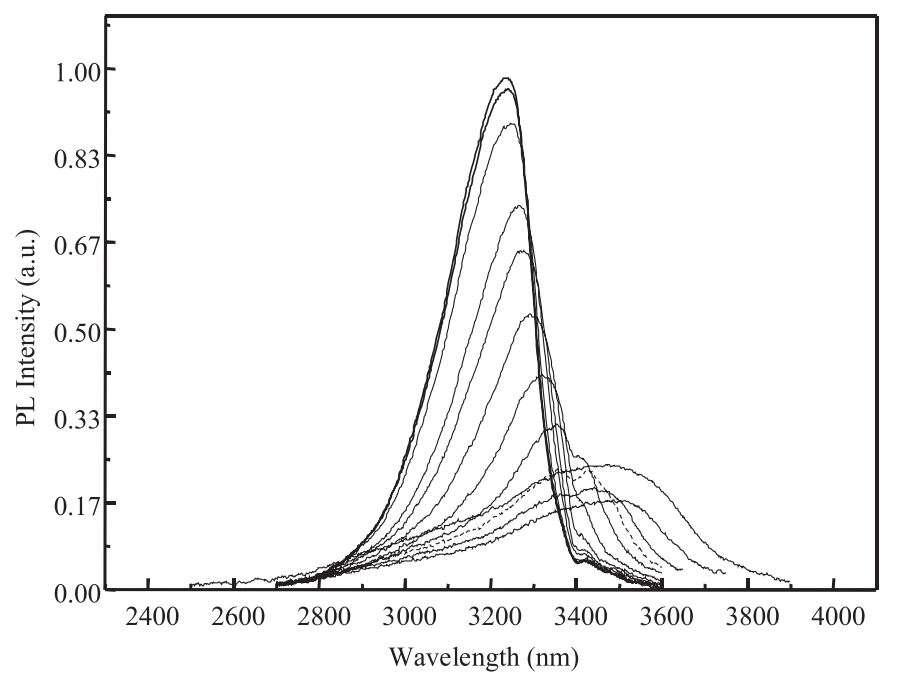

Fig. 8.8. Photoluminescence spectra of a $500 \mathrm{~nm}$ thick InAsSb layer for temperatures from 78 to $300 \mathrm{~K}$. The maximum intensity is at $78 \mathrm{~K}$ and decreases with increasing temperature in increments of $20 \mathrm{~K}$.

Electrochemical capacitance-voltage (ECV) was used to obtain the carrier concentration profile. The temperature and flow rate dependence on the carrier concentration is shown in Figures 8.17 and 8.18.

DeZn is used as $p$-type dopant. The bubbler pressure and temperature for DeZn were 700 torr and $-5^{\circ} \mathrm{C}$. Electrochemical capacitance-voltage (ECV) was used to obtain the carrier concentration profile.

Figures 8.19 and 8.20 show the experimental relationship between $p$-type carrier concentration and zinc flow rate and growth temperature.

\section{$8.2 \ln \mathrm{AsSb} / \operatorname{In} \mathrm{AsSbP} / \operatorname{In} \mathrm{As}$ double heterostructure lasers}

Double heterostructure InAsSbP/InAsSb/InAs were grown on (100)-oriented Te-doped InAs substrates by LP-MOCVD [52]. The heterostructure consists of a $1.0 \mu \mathrm{m}$ thick undoped ternary active region $\mathrm{InAs}_{0.92} \mathrm{Sb}_{0.08}$ sandwiched between two $1.5 \mu \mathrm{m}$ thick InAsSbP confinement layers: Sn doped $\left(\sim 10^{18} \mathrm{~cm}^{-3}\right)$ and $\mathrm{Zn}$ doped $\left(\sim 10^{18} \mathrm{~cm}^{-3}\right)$, respectively, and a $p^{+}$-doped InAs cap layer.

The structural quality is measured via X-ray diffraction and a typical scan is shown in Figure 8.21. The FWHM of the overlapping peaks is 120 arcs which indicates high quality.

Broad-area laser diodes with a stripe width of $100 \mu \mathrm{m}$ were fabricated using lift-off process, in which the $p^{+}$InAs cap layer is etched away between the stripes. After Ohmic contact deposition, individual diodes were cleaved and mounted $p$-side up on copper heat sinks by indium bonding. Uncoated diodes with cavity length varying from 200 to $1200 \mu \mathrm{m}$ were prepared.

Light-current characteristics were measured in both $\mathrm{cw}$ and pulse mode (pulse width $4-7 \mathrm{~ms}$, repetition rate $200 \mathrm{~Hz}$ ) operation for several lasers mounted inside a cryostat with temperatures varying between 77 and $300 \mathrm{~K}$, using a Judson J10D InSb photodetector. Figure 8.22 shows total output power of $260 \mathrm{~mW}$ per two facets from several uncoated diode lasers at injection current of $6 \mathrm{~A}$. As shown in the inset of Figure 8.22, these DH lasers exhibited multiple longitudinal modes centered at $3.2 \mu \mathrm{m}$ at $77 \mathrm{~K}$. The lowest values of threshold current density amounted to $40 \mathrm{~A} / \mathrm{cm}^{2}$ with differential efficiency of $18 \%$ at $77 \mathrm{~K}$. For cw operation, maximum output power of $60 \mathrm{~mW}$ per two facets was achieved at $77 \mathrm{~K}$ at injection current of 2 A (Fig. 8.23) with low series resistance of $0.23 \Omega$ at $77 \mathrm{~K}$. The inset of Figure 8.23 shows the typical $\mathrm{DH}$ band structure of these $100 \mu \mathrm{m}$ wide lasers. It was observed in several diodes under $\mathrm{cw}$ operation that the output power begins to saturate at higher currents as shown in Figure 8.23, probably because of an increase in junction temperature. The characteristic temperature for these diodes was determined to be $T_{0}=42 \mathrm{~K}$ from the temperature dependence of the threshold current density measured up to $130 \mathrm{~K}$. Maximum operating temperature up to $220 \mathrm{~K}$ was achieved in pulse mode operation.

\subsection{Growth, characterization, and measurement of strained-layer superlattice lasers}

Despite the need for coherent emitters in the $4-5 \mu \mathrm{m}$ wavelength region, additional increases in emission wavelength have been found to be difficult with conventional interband emitter (DH and MQW) structures. For DH lasers emitting at $\lambda>3.4 \mu \mathrm{m}$, the misfit dislocations between the $\operatorname{InAs}_{x} \mathrm{Sb}_{y} \mathrm{P}_{1-x-y}$ cladding and $\mathrm{InAs}_{x} \mathrm{Sb}_{1-x}$ active region are increased to levels that prohibit oscillation. MQW emitters have demonstrated the ability to emit spontaneously covering the entire $3-5 \mu \mathrm{m}$ region. This is 


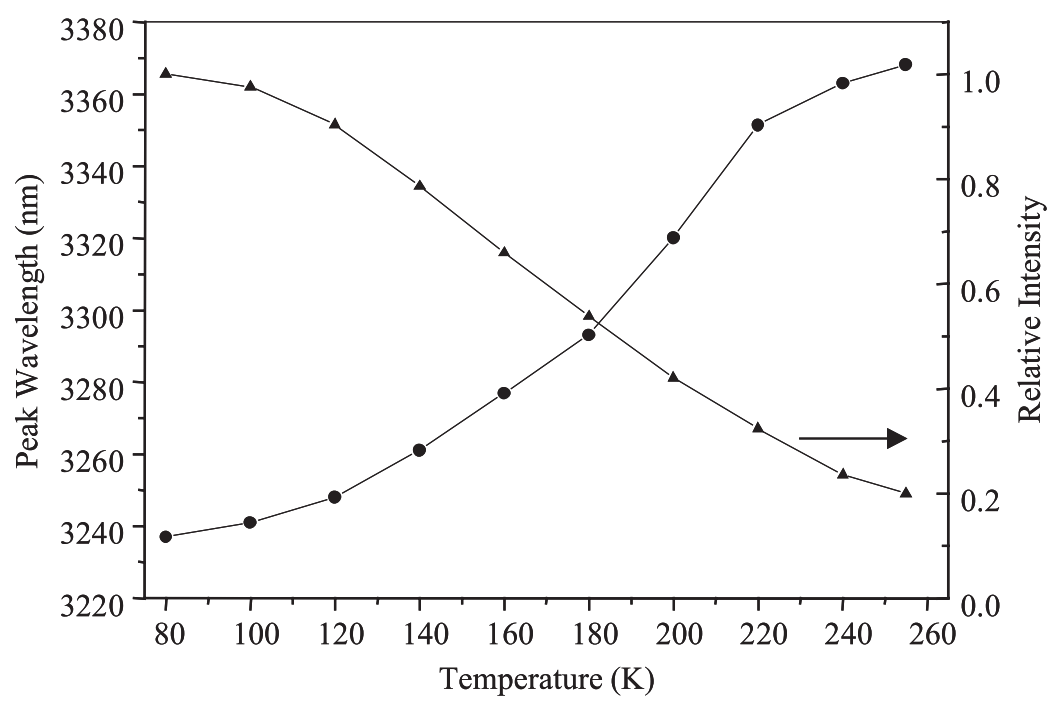

Fig. 8.9. Photoluminescence wavelength and normalized peak intensity of a $500 \mathrm{~nm}$ thick InAsSb epilayer versus temperature.

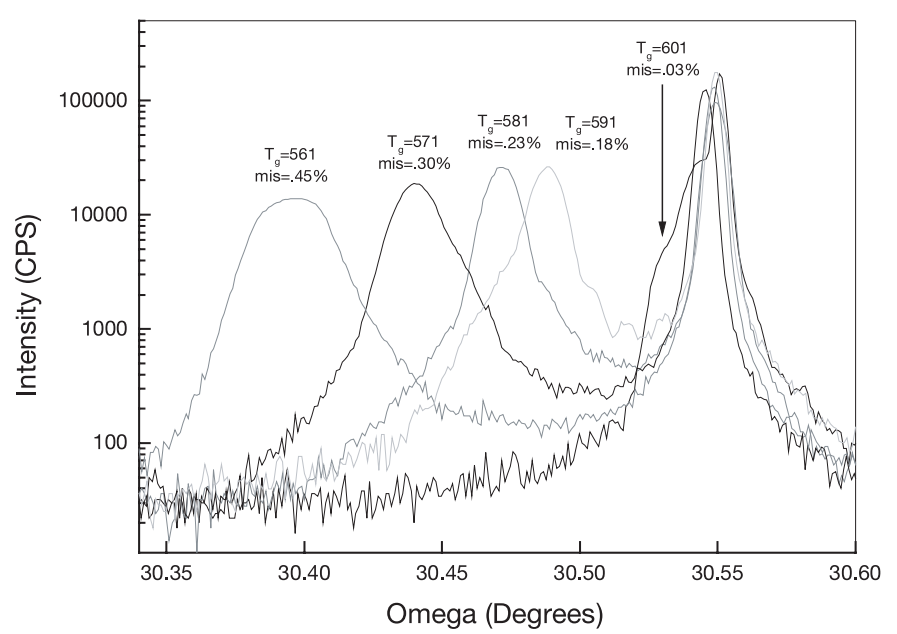

Fig. 8.10. X-ray of 5 InAsSbP epilayers grown at different temperatures.

shown below in Figure 8.24 in which the antimony composition was varied in the quantum wells (InAsSb) of an InAs/InAs ${ }_{x} \mathrm{Sb}_{1-x}$ MQW structure. The cladding region was InAsSbP in all five structures.

This is a result of the fact that MQW structures can accommodate a larger percent mismatch between active and barrier regions than DH structures as long as the thickness of the quantum well is thinner than the critical thickness. The critical thickness of InAs $/ \operatorname{InAs}_{x} \mathrm{Sb}_{1-x}$ has been calculated using the Matthews-Blakeslee and PeopleBean relationships [53,54]. The experimental results are much better fit to the People-Bean relationship which is shown in Figure 8.25.

Also, the emission wavelength for bulk InAsSb is shown in Figure 8.26. It shows that around 20\% antimony must be incorporated into a bulk active region for emission up to $5 \mu \mathrm{m}$.

From above, critical thickness of $\operatorname{InAs}_{0.8} \mathrm{Sb}_{0.2}$ is approximately $100 \AA$. However using these dimensions, the

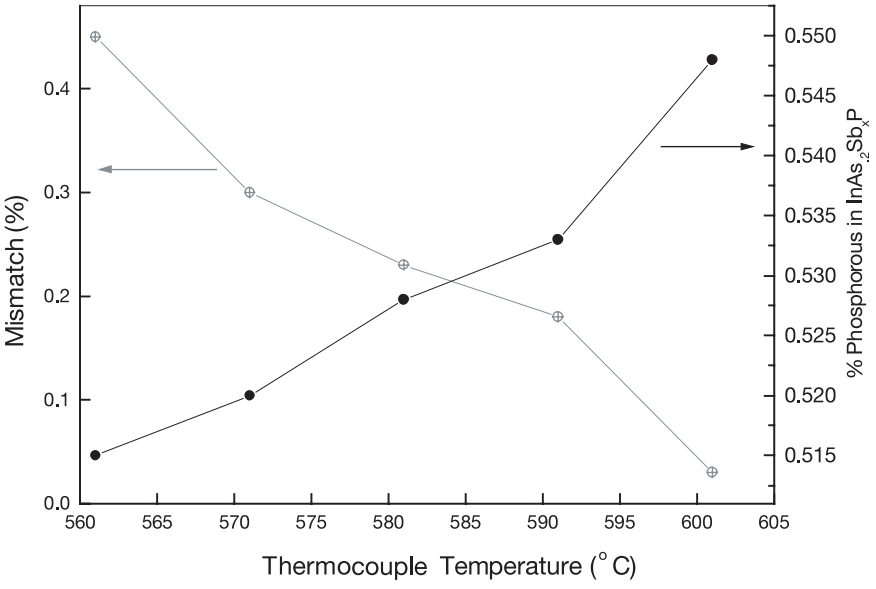

Fig. 8.11. Mismatch from $\mathrm{InAs}$ and percentage $\mathrm{P}$ in InAs $\mathrm{s}_{0.2} \mathrm{SbP}$ versus growth temperature.

electrical and optical confinements and gain inherent in active region are not enough to allow lasing in a MQW structure. This has been verified experimentally.

Strained-layer superlattice (SLS) lasers which benefit from better optical confinement and more emission wavelength flexibility than offered by a DH structure and a larger gain region than lasers based on a MQW active region are good candidates for high power and high temperature emission between 3.8 and $5 \mu \mathrm{m}$. High temperature operation is enhanced with SLS lasers by the suppression of Auger recombination mechanisms with the large number of interfaces and compressive strain inherent with these lasers [55].

As an example, Figure 8.27 shows the calculated layer thickness (the well thickness is equal to the barrier thickness) dependence of emission wavelength for an $\operatorname{InAs}_{0.95} \mathrm{P}_{0.05} / \mathrm{InAs}_{0.75} \mathrm{Sb}_{0.25}$ SLS structure which has $0.8 \%$ mismatch from InAs. The Kronig-Penney model, including the strain induced band edge shifting, has been used for the calculation. The flat dotted line represents 


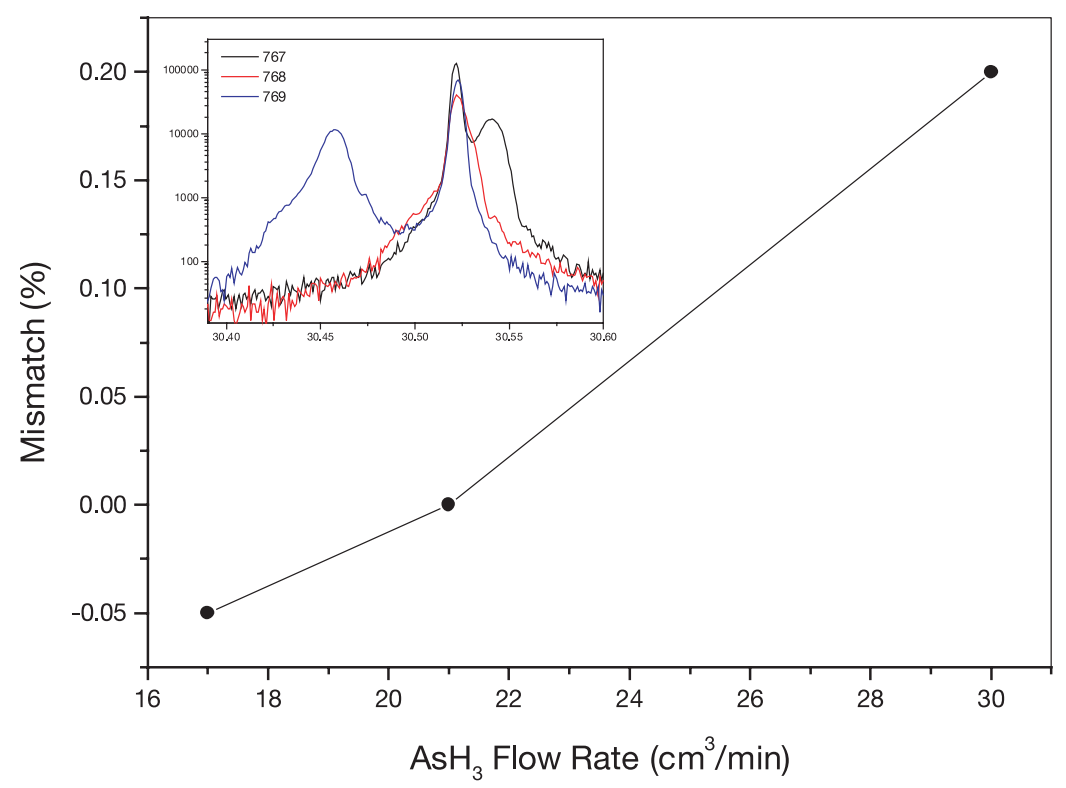

Fig. 8.12. Mismatch from InAs substrate to an InAsSbP layer as a function of different $\mathrm{AsH}_{3}$ flow rates.

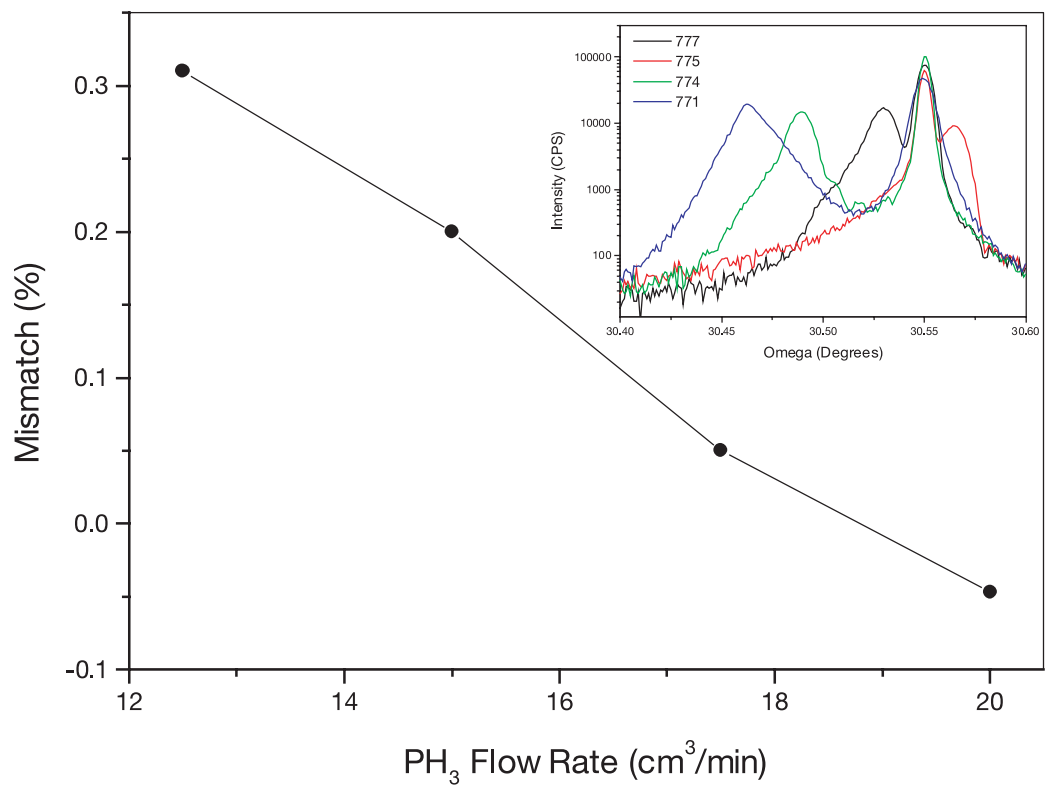

Fig. 8.13. Mismatch of InAsSbP versus $\mathrm{PH}_{3}$ flow rate. The X-ray diffraction spectra for the four samples is shown in the inset.

the emission wavelength of an InAsSb alloy with $0.8 \%$ mismatch from InAs which can only have an emission wavelength at $\lambda=3.7 \mu \mathrm{m}$. Alternatively, by changing the layer thickness in the superlattice, the emission wavelength can be tuned from 3.3 to $4.5 \mu \mathrm{m}$ independent of its lattice constant. Therefore, the SLS structure allows the structure to have longer emission wavelength $(>4 \mu \mathrm{m})$ with a non-prohibitive mismatch from InAs substrate.

There are three superlattice material alloys that have the ability to emit between $3.8-5 \mu \mathrm{m}$ : $\operatorname{InAs}_{1-x} \mathrm{Sb}_{x} / \operatorname{InAs}_{1-y} \mathrm{P}_{y}, \quad \operatorname{InAs} / \operatorname{InAs}_{1-x} \mathrm{Sb}_{x}, \quad$ and $\mathrm{InAs}_{1-x} \mathrm{Sb}_{x} / \mathrm{InAs}_{1-y} \mathrm{Sb}_{y}$.

\subsubsection{InAsSb/InAsP/InAs strained layer superlattice lasers}

Strained-layer superlattice laser structures were grown on (100) oriented Te-doped InAs substrates in a vertical metal MOCVD reactor. The active region consisted of $\operatorname{InAs}_{1-x} \mathrm{Sb}_{x} / \operatorname{InAs}_{1-y} \mathrm{P}_{y}$ superlattice with $x \approx 0.75$ and $y \approx 0.05$. The barrier and the well of the superlattice have the same thickness. The total period thickness is $100 \AA$ and 80 periods were used. For the entire laser structure, $1.1 \mu \mathrm{m}$ InAsSbP cladding layers were grown before and after the superlattice growth. These layers were grown with the same doping profile as those used for double heterostructure lasers at the optimized temperature of $585{ }^{\circ} \mathrm{C}$. Since the composition of both ternary and 


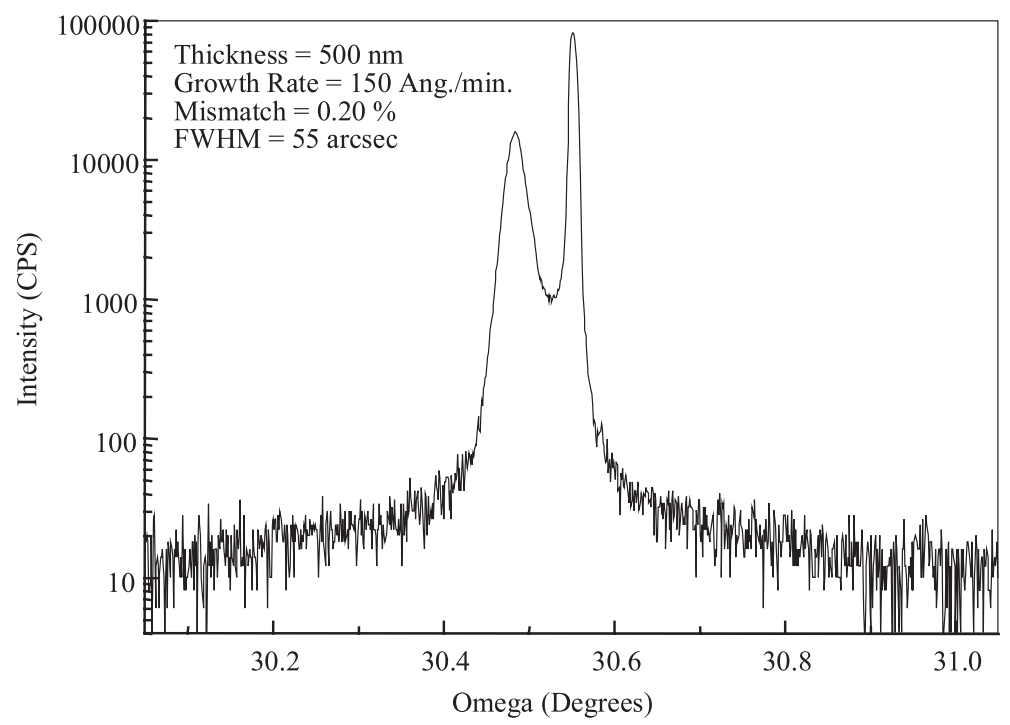

Fig. 8.14. X-ray diffraction spectra for a $500 \mathrm{~nm}$ thick InAsSbP layer.

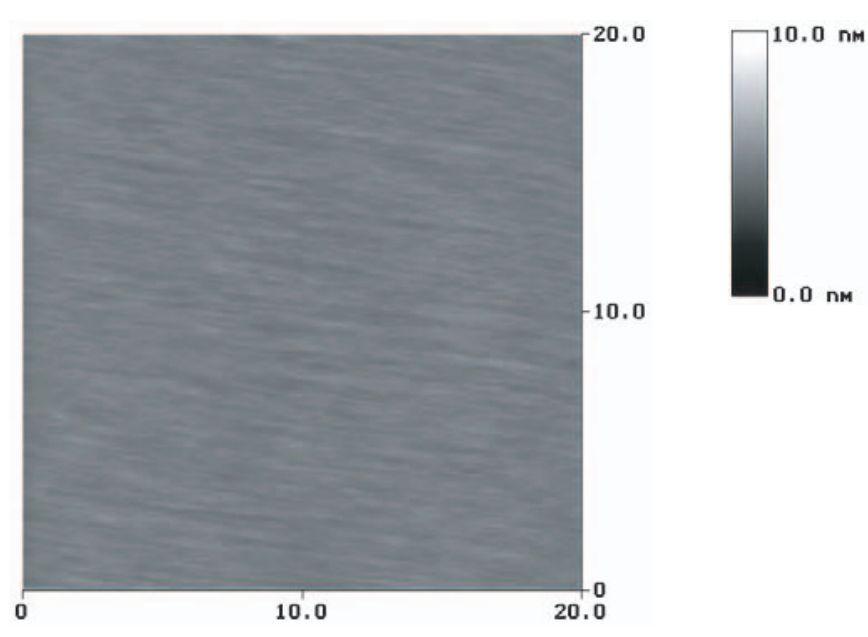

Fig. 8.15. AFM image of a $500 \mathrm{~nm}$ thick InAsSbP layer.

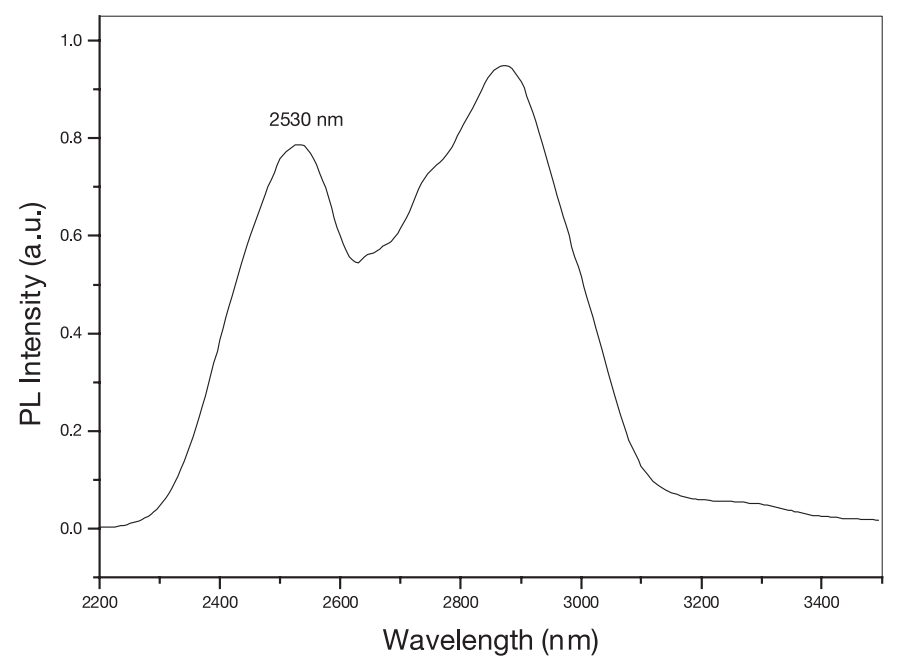

Fig. 8.16. Photoluminescence of InAsSbP on InAs at $78 \mathrm{~K}$. The InAsSbP peak is at $2530 \mathrm{~nm}$.

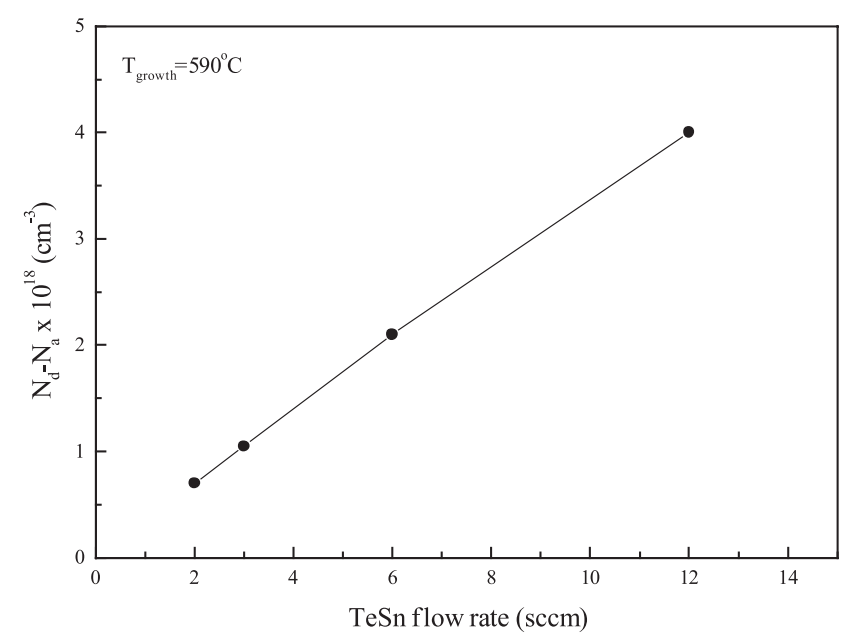

Fig. 8.17. $N$-type carrier concentration versus tin flow rate.

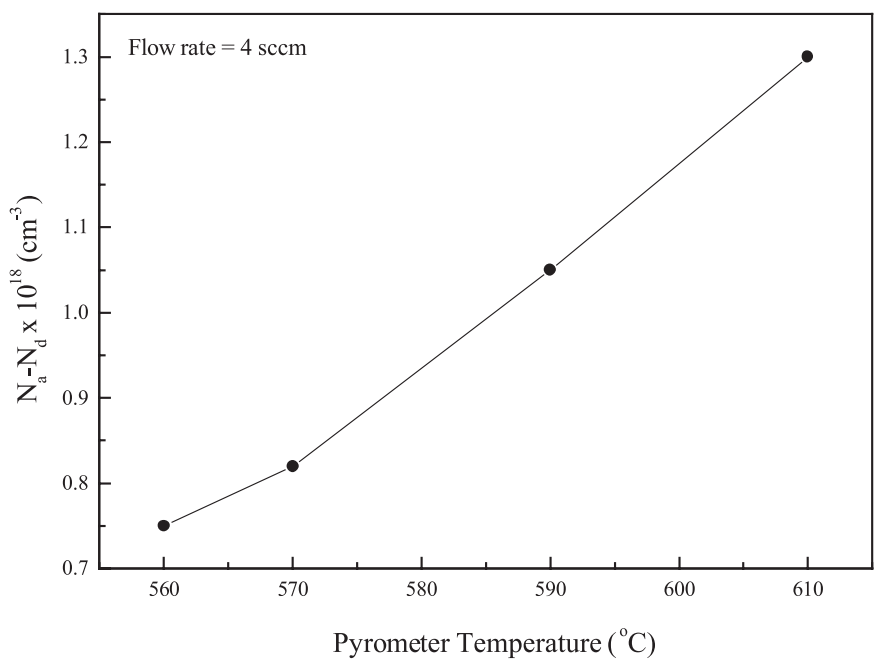

Fig. 8.18. $N$-type carrier concentration versus growth temperature. 


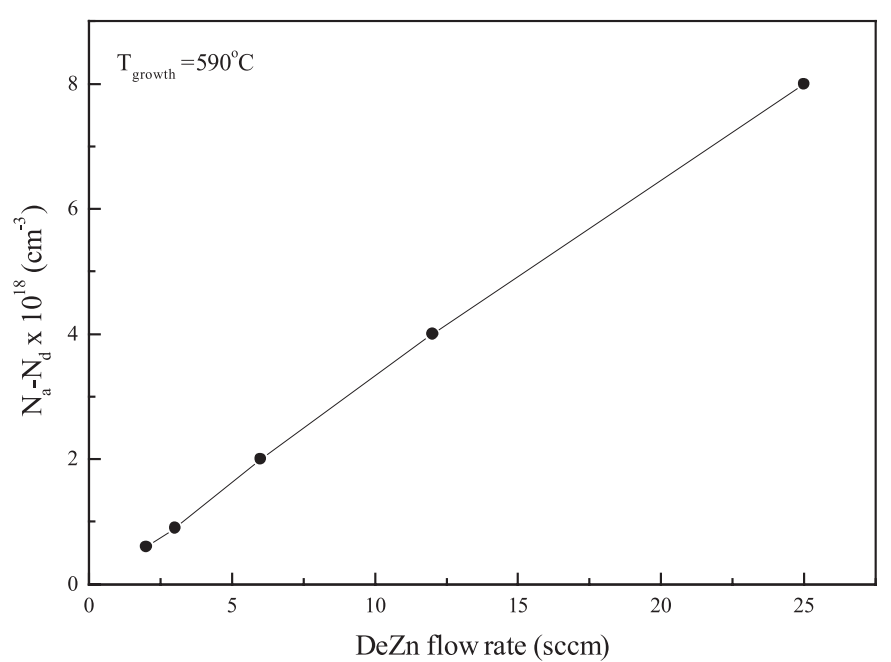

Fig. 8.19. $p$-type carrier concentration versus zinc flow rate.

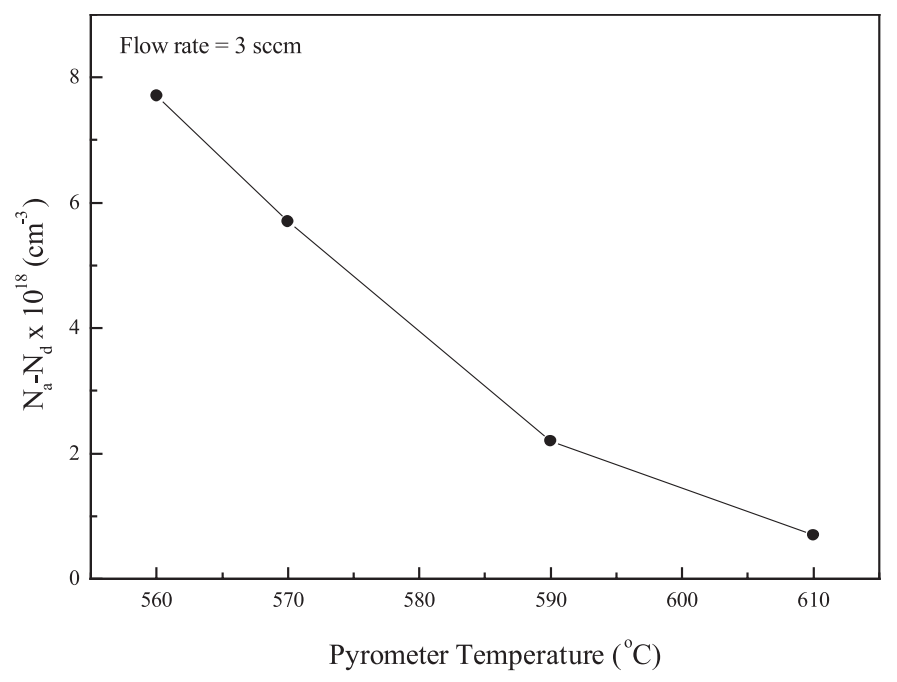

Fig. 8.20. $P$-type carrier concentration as a function of growth temperature.

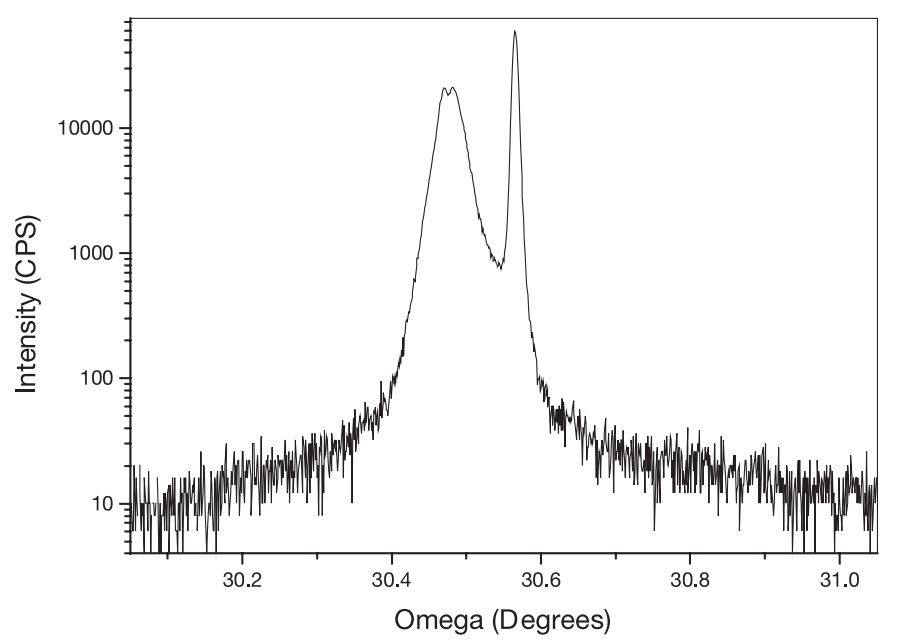

Fig. 8.21. Typical X-ray spectra for a InAsSb/InAsSbP/InAs double heterostructure laser.

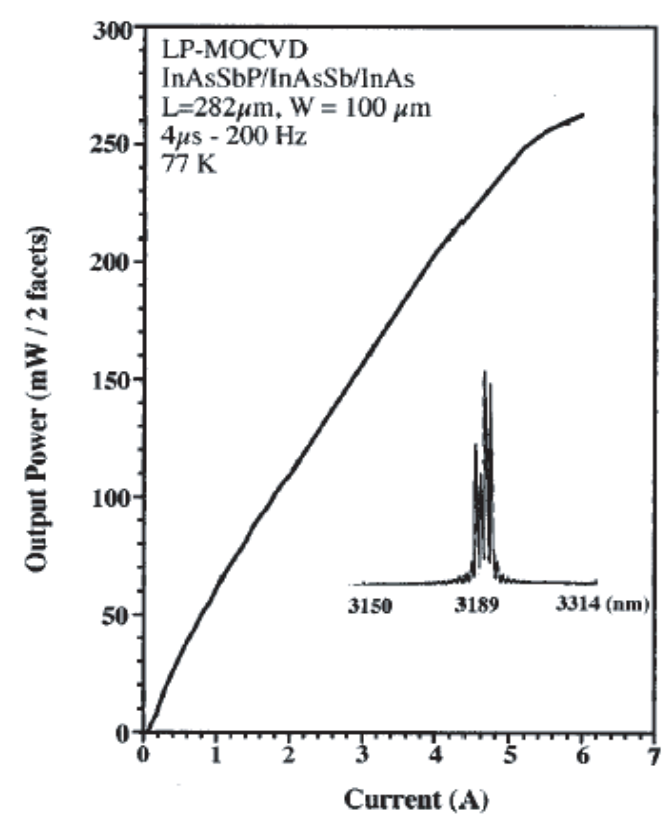

Fig. 8.22. Light output vs. pulsed current for an InAsSbP/InAsSb/InAs DH laser diode at $77 \mathrm{~K}$. The inset shows the emission wavelength at $77 \mathrm{~K}$.

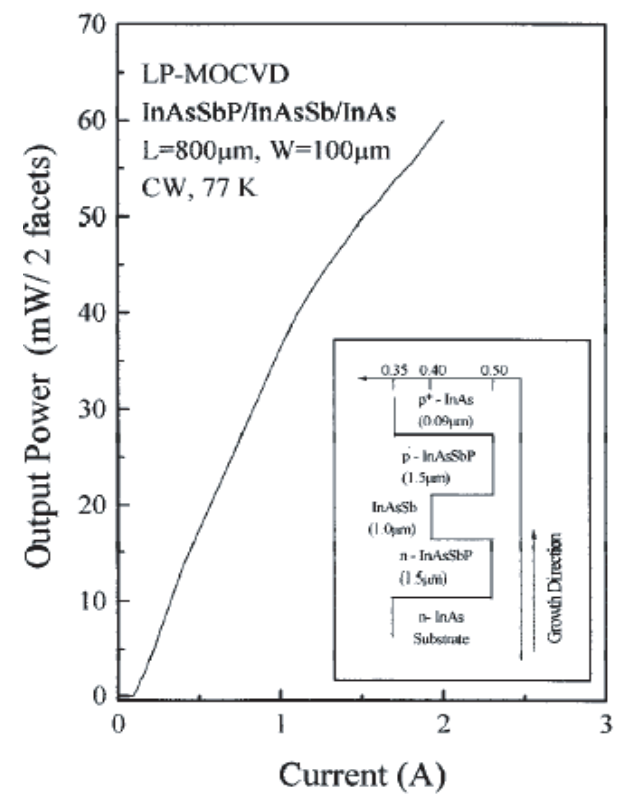

Fig. 8.23. Light output vs. current for $\mathrm{cw}$ operation of an InAsSbP/InAsSb/ InAs DH uncoated diode laser at $77 \mathrm{~K}$. The inset shows the typical DH InAsSbP/InAsSb/InAs diode lasers.

quaternary materials change with temperature, InAs was grown when the temperature was ramped between the cladding and superlattice. At the conclusion of the $n$ type InAsSbP cladding and InAsP/InAsSb superlattice region, InAs was grown while the temperature was ramped linearly between 585 and $560{ }^{\circ} \mathrm{C}$. The temperature was stabilized for two minutes after the ramping period. A growth rate of $100 \AA / \mathrm{min}$ was used to minimize the total thickness of InAs. The structure was completed with a 


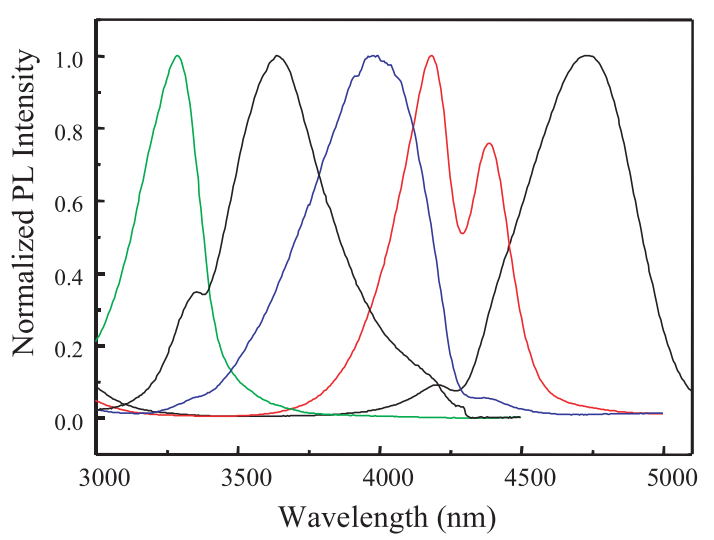

Fig. 8.24. PL spectra for $5 \mathrm{MQW}$ structures demonstrating the ability to cover the entire $3-5 \mu \mathrm{m}$ wavelength region.

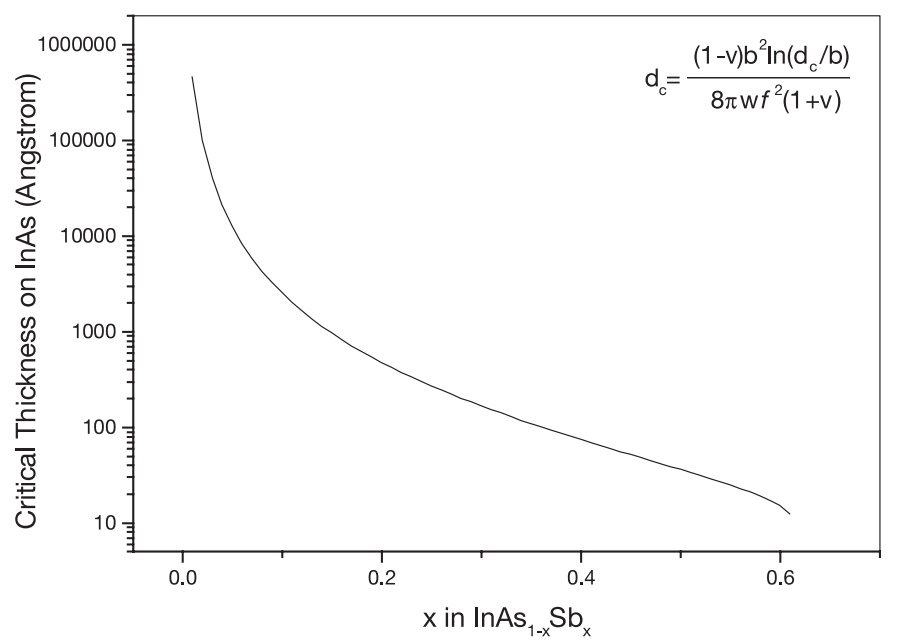

Fig. 8.25. Calculated critical thickness of $\operatorname{InAs}_{1-x} \mathrm{Sb}_{x}$ on InAs.

$100 \mathrm{~nm} p^{+}$InAs cap layer and is shown schematically in Figure 8.28.

The morphology of the laser structure was mirrorlike with misfit dislocations similar to those for double heterostructure lasers. The X-ray diffraction spectra has been measured and compared with theoretical calculations of the spectra. They are shown in Figure 8.29. The high crystalline quality of the strained superlattice structure is confirmed via X-ray measurements and by the three orders of satellite peaks observed in the spectra. These measurements were used to determine the average strain of the superlattice to be $0.79 \%$. This is compared to the theoretically predicted mismatch of $0.78 \%$. The peaks are well aligned and are periodic which demonstrates that the period is the designed thickness and is consistent throughout the growth. The measured period from the superlattice satellite peaks is $98 \AA$ while the design period was $100 \AA$. However, the FWHM is far greater for the measured spectra, which is most likely due to an interfacial layer in between the two layers.

The photoluminescence versus temperature is also provided below in Figure 8.30. The peak emission wavelength is $3.95 \mu \mathrm{m}$ with a narrow FWHM of only $29 \mathrm{meV}$ at $77 \mathrm{~K}$.

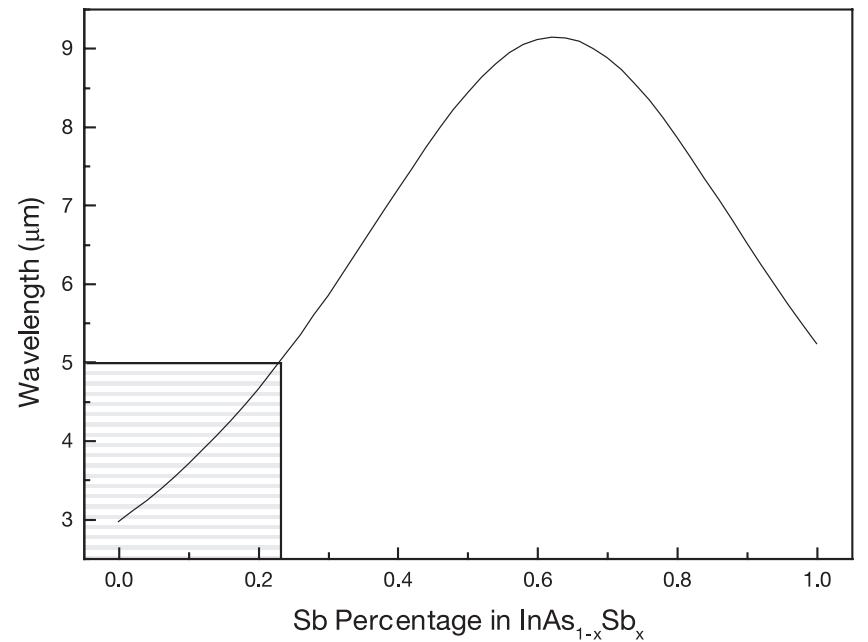

Fig. 8.26. Emission wavelength for bulk $\operatorname{InAs}_{1-x} \mathrm{Sb}_{x}$ as a function of antimony composition at $78 \mathrm{~K}$. The shaded area shows the required compositions to cover the $3-5 \mu \mathrm{m}$ region.

Broad-area laser diodes with a stripe width of $100 \mu \mathrm{m}$ were fabricated using a lift-off process, in which the $p^{+}$ InAs contact cap layer is etched away between the stripes. After Ohmic contacts were deposited, individual diodes were cleaved and mounted $p$-side up on copper heat sinks by indium bonding. Uncoated laser diodes of cavity lengths varying between 400 and $1800 \mu \mathrm{m}$ were prepared, mounted in a cryostat and then measured.

Figure 8.31 shows the $P I$ curve and emitting spectra for the laser under pulsed operation at $T=100 \mathrm{~K}$. The output power was measured to be $546 \mathrm{~mW}$ with a corresponding threshold current density of $100 \mathrm{~A} / \mathrm{cm}^{2}$ and a differential efficiency $30 \%$ for the cavity length of $1800 \mu \mathrm{m}$. From the emitting spectra in the inset of Figure 8.29, several longitudinal modes, centered around $4.08 \mu \mathrm{m}$, can be observed. Continuous wave measurements, shown in Figure 8.30, resulted in an output power of $94 \mathrm{~mW}$ per two facets with a differential efficiency of over $30 \%$. As mentioned previously, at higher currents, Joule heating becomes significant and is most likely the predominant limiting mechanism at these high powers [56]. Additionally, from the emitting spectra dependence on injection current and temperature, the actual temperature of the junction is estimated to be $20 \mathrm{~K}$ greater than that of the heat sink. To the best of our knowledge, this is the lowest reported threshold current density for electrically injected lasers operating above $4 \mu \mathrm{m}$. The highest operating temperature for this laser was $125 \mathrm{~K}$ and the threshold current density dependence upon temperature gave a $T_{o}=27 \mathrm{~K}$. The perpendicular far-field divergence was confirmed by several measurements to be $28^{\circ}$.

\subsubsection{InAsSb/InAs(Sb)/InAs strained layer superlattice lasers}

The InAsSb/InAs, InAs ${ }_{1-x} \mathrm{Sb}_{x} / \operatorname{InAs}_{y} \mathrm{Sb}_{1-y}$ superlattice active regions do not suffer from the problems resulting 


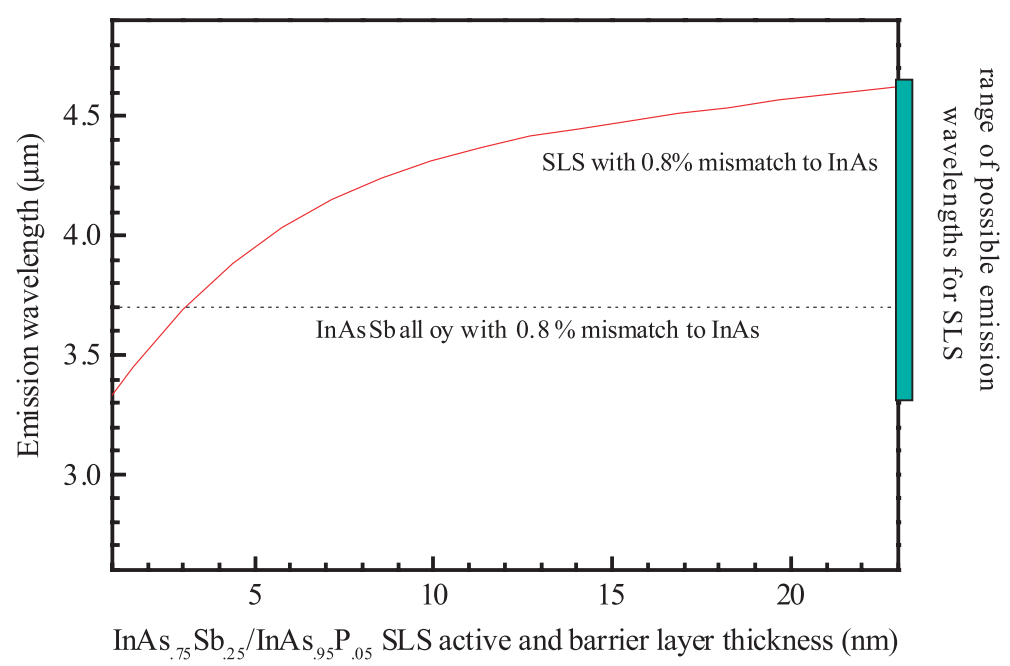

Fig. 8.27. Calculated layer thickness (in our design the well thickness is equal to the barrier thickness) dependence of emission wavelength for an $\operatorname{InAs}_{0.95} \mathrm{P}_{0.05} / \mathrm{InAs}_{0.75} \mathrm{Sb}_{0.25} \mathrm{SLS}$ structure which has $0.8 \%$ mismatch from InAs.

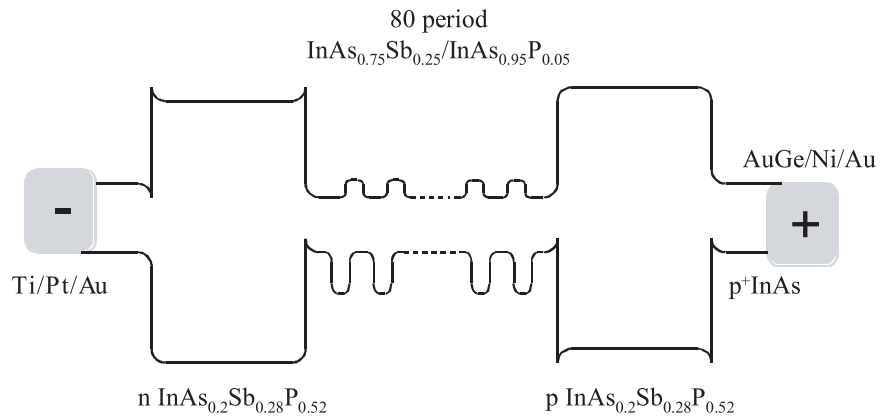

Fig. 8.28. Schematic band alignments of a InAsSb/InAsP strained-layer superlattice injection laser.

from the InAsP and have the advantages of large emission tunability because of the type-II band alignment of InAsSb and InAs. However, there are disadvantages for these two superlattice systems. First, associated with the type-II alignment is a reduced wave function overlap resulting in a lower optical matrix element and finally a reduced radiative transition probability. Additionally, since InAsSb is compressively strained to InAs, the superlattices will also be strained.

For InAsSb/InAsP SLS high values of $x$ are required because of the large conduction band offset that InAsP creates in the material. Since InAs and InAsSb are used as the barrier materials in InAsSb/InAsSb and InAs/InAsSb superlattice regions, much smaller values of $x$ are required. This will allow for higher growth temperatures that reside closer to the optimum growth temperature. Calculations show that for superlattices with the same thickness for well and barrier, $x$ is no greater than $15 \%$. Furthermore, for both of the active region superlattices, $100 \AA$ thick layers will be grown. This ensures that they are below the critical thickness and that the number of misfit dislocations is kept to a minimum.

Despite the fact that the growth temperature is closer to the InAsSb optimum growth temperature, the switch-

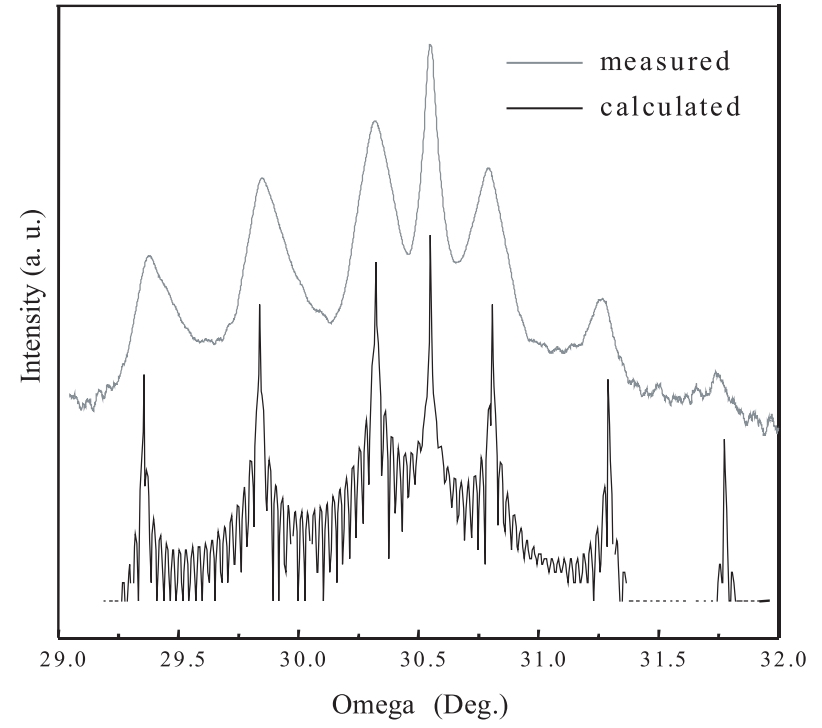

Fig. 8.29. Measured and calculated X-ray diffraction spectra for an InAsSb/InAsP superlattice laser.

ing routine is more critical for these superlattices than for the InAsSb/InAsP superlattices. Since antimony has a long residence time during the growth process, a balance must be made between the growth interruption or change in antimony flow rates. Too long an interval translates into complete antimony removal, which results in an arsenic rich InAsSb interface. Too short a growth interruption will result in non-abrupt interfaces. The growths of the barriers for both of the superlattices focused upon are routine. The barriers are either InAs or InAsSb with an antimony composition similar to that used for the active region for double heterostructure lasers.

First, the active well materials were grown. The X-ray for a sample corresponding to an antimony incorporation percentage of $9 \%$ is shown below in Figure 8.31. 


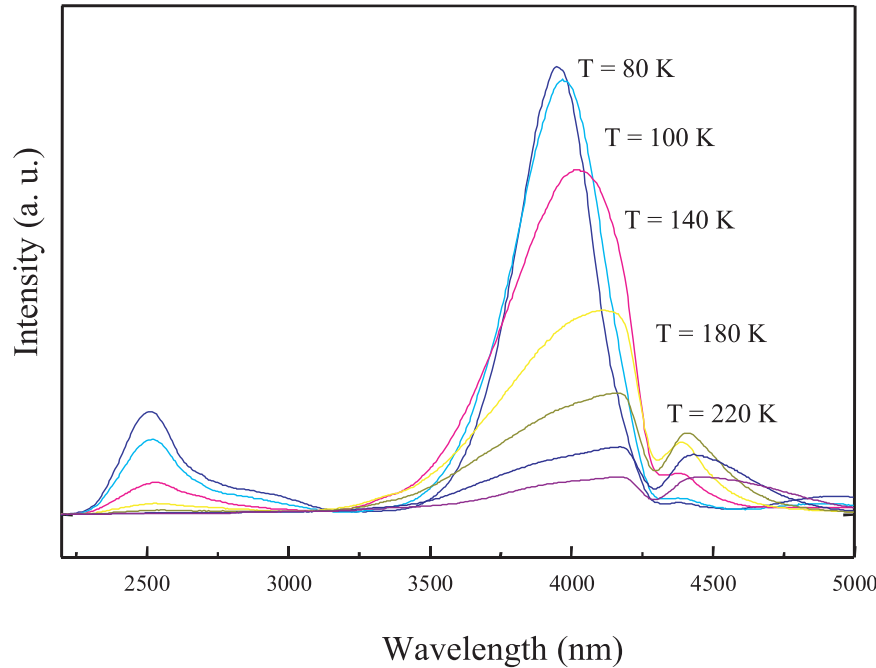

Fig. 8.30. PL spectra for an InAsSb/InAsP superlattice laser.

Finally, the InAs and InAsSb barrier layers were grown. For InAs homoepitaxy, the characterization is excellent as expected and described previously. The X-ray FWHM is limited by the resolution of the diffractometer and the AFM confirms atomically flat layers. The same is true for the $\mathrm{InAs}_{0.95} \mathrm{Sb}_{0.05}$ epilayers. The AFM characterization for a $400 \mathrm{~nm}$ sample is also very good; the atomic steps resulting from the miscut of the substrate are seen. The X-ray FWHM is $\sim 45$ arcs and the roughness of $10 \times 10 \mu \mathrm{m}$ scan is $4 \AA$.

The photoluminescence versus temperature spectra measurements are provided below in Figure 8.34. The peak emission wavelength is $3.95 \mu \mathrm{m}$ with a narrow FWHM of $29 \mathrm{meV}$. InAsSbP cladding regions, with the same composition used for the previously described lasers, were used for confinement. The peak emission wavelength at $78 \mathrm{~K}$ is $\sim 4.1 \mu \mathrm{m}$ and the FWHM is slightly eroded by $\mathrm{CO}_{2}$ absorption at $4.2 \mu \mathrm{m}$.

The X-ray and PL spectra for a typical InAsSb/InAs superlattice is shown below in Figures 8.35 and 8.36. The decay of PL intensity with increasing temperature is comparable with other double heterostructure lasers with wavelengths near $\lambda=3.2 \mu \mathrm{m}$.

Broad-area laser diodes with a width of $100 \mu \mathrm{m}$ were fabricated. Optical output power-injection current characteristics were measured in both $\mathrm{cw}$ and pulsed mode operation for lasers mounted inside a cryostat with temperatures varying between 77 and $140 \mathrm{~K}$.

For InAs/InAsSb SLS lasers designed to emit at $3.8 \mu \mathrm{m}, P I$ measurements revealed a threshold current density of $67 \mathrm{~A} / \mathrm{cm}^{2}$ and a differential efficiency of $38 \%$. These performance characteristics are similar to those of the DH lasers previously reported. The pulsed PI is shown in Figure 8.37. The differential efficiency was observed to be constant over the entire range of operating temperatures measured. The highest operating temperature was measured to be $120 \mathrm{~K}$. Under continuous wave operation, these diodes displayed output powers as high as $300 \mathrm{~mW}$ at $80 \mathrm{~K}$. This is the highest reported $\mathrm{cw}$ output power in this wavelength range for electrically injected lasers. The cw PI curve is shown in Figure 8.38. Furthermore, perpendicular far-field divergence angles and series resistance measurements revealed values of $34^{\circ}$ and $0.3 \Omega$ respectively at $78 \mathrm{~K}$. The transparent current density was found to be $\sim 47 \mathrm{~A} / \mathrm{cm}^{2}$ from the threshold current density dependence on cavity length. This compares favorably to the values measured for double heterostructure lasers considering the complexity of these lasers. The internal loss was also calculated to be $16 \mathrm{~cm}^{-1}$.

Even longer emission has been achieved with InAsSb/InAsSb SLS lasers. Figure 8.39 shows the $P I$ and spectra of an InAsSb/InAsSb SLS laser diode emitting multiple longitudinal modes centered at $4.26 \mu \mathrm{m}$. For lasers with an emission wavelength of $4.26 \mu \mathrm{m}$, we have measured an output power from a single $100 \mu \mathrm{m}$ aperture with $T=80 \mathrm{~K}$ of $165 \mathrm{~mW}$. PI measurements revealed a threshold current density of $300 \mathrm{~A} / \mathrm{cm}^{2}$ and a differential efficiency of $20 \%$. The differential efficiency was observed to be constant over the entire range of operating temperatures measured. A characteristic temperature $T_{0}=40 \mathrm{~K}$ was measured from the dependence of threshold current density on temperature. The highest operating temperature was measured to be $140 \mathrm{~K}$. Furthermore, perpendicular far-field divergence angles and series resistance measurements revealed values of $43^{\circ}$ and $0.3 \Omega$ respectively at $77 \mathrm{~K}$.

Increasing the antimony composition with the superlattice has allowed lasers with an emission wavelength of $4.46 \mu \mathrm{m}$ to be fabricated. Despite the longer emission wavelengths and therefore the higher Auger and free carrier absorption losses, output powers from a single $100 \mu \mathrm{m}$ aperture of $199 \mathrm{~mW}$ with $T=79 \mathrm{~K}$ have been measured This $P I$ curve is shown in Figure 8.40. The threshold current density was only $160 \mathrm{~A} / \mathrm{cm}^{2}$ its dependence upon temperature gave a $T_{0}$ of $16 \mathrm{~K}$.

Single stripe InAsSb/InAsSb lasers emitting at $\lambda=$ $4.76 \mu \mathrm{m}$ have also been measured. A maximum output power of $92 \mathrm{~mW}$ under pulsed mode operation for $4.3 \mathrm{~A}$ injection current at $79 \mathrm{~K}$ is reported (Fig. 8.41). The emitting spectrum reveals multiple longitudinal modes centered at $4.76 \mu \mathrm{m}$. PI measurements revealed a threshold current density of $393 \mathrm{~A} / \mathrm{cm}^{2}$ and a differential efficiency of over $10 \%$. These lasers have also been fabricated into laser bars and have displayed a maximum output power of $230 \mathrm{~mW}$ for a total aperture of $1 \mathrm{~mm}$. This is the longest reported emission wavelength for injection interband lasers with this material system.

\section{$9 \mathrm{GaSb} / \mathrm{InAs}$ type II superlattice for IR photodetectors}

\subsection{Introduction}

Recently, type-II superlattices have been proposed as an alternative to $\mathrm{HgCdTe}$ for the strategic windows of $3-5 \mu \mathrm{m}$ and $8-12 \mu \mathrm{m}$ as well as longer wavelengths. Type-II superlattices are constructed from the III-V material system, 


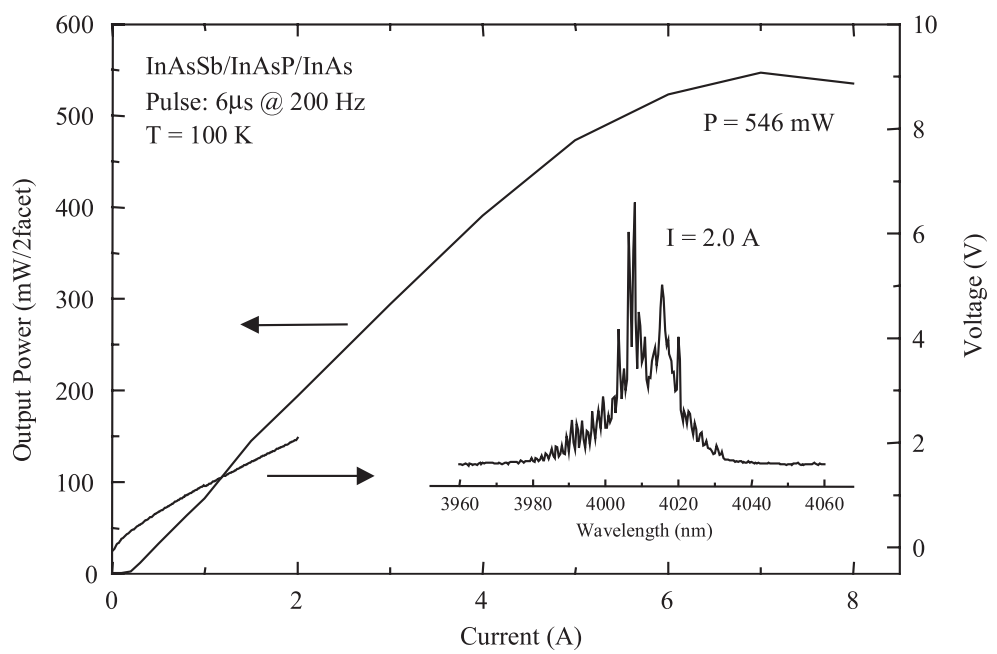

Fig. 8.31. Light output vs. pulsed current of a InAsSb/InAsP strained-layer superlattice laser at $T=100 \mathrm{~K}$. The inset shows the emission wavelength at $80 \mathrm{~K}$.

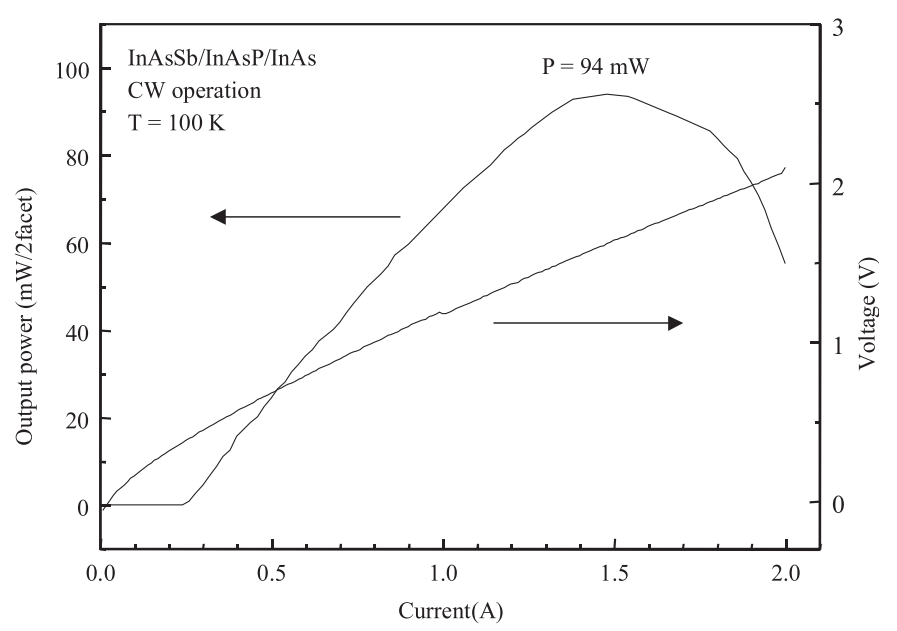

Fig. 8.32. Light output vs. current and current versus voltage curve for $\mathrm{cw}$ operation of a InAsSb/InAsP strained-layer superlattice laser at $T=100 \mathrm{~K}$.

and hence they have much better mechanical properties and material uniformity than $\mathrm{HgCdTe}$. The electron effective mass in these superlattices is higher than the electron effective mass in $\mathrm{HgCdTe}$ and InAsSb, therefore the tunneling current is less. The bandgap of the superlattice can be changed to cover a wide IR range (from $\sim 2 \mu \mathrm{m}$ to above $25 \mu \mathrm{m}$ ) by changing the thickness of the layers rather than the material composition. Also, in comparison to quantum well infrared photodetectors (QWIPs), typeII superlattices have a much higher quantum efficiency while they do not require surface gratings for absorption of normal incident light.

Type-II band alignment and some of its interesting physical behavior were originally suggested by Sai-Halasz and L. Esaki [57] in 1977. Soon after that they reported the optical absorption of type-II superlattices [58] and later the semimetal behavior of the superlattice [59]. The applications of such a superlattice were proposed several

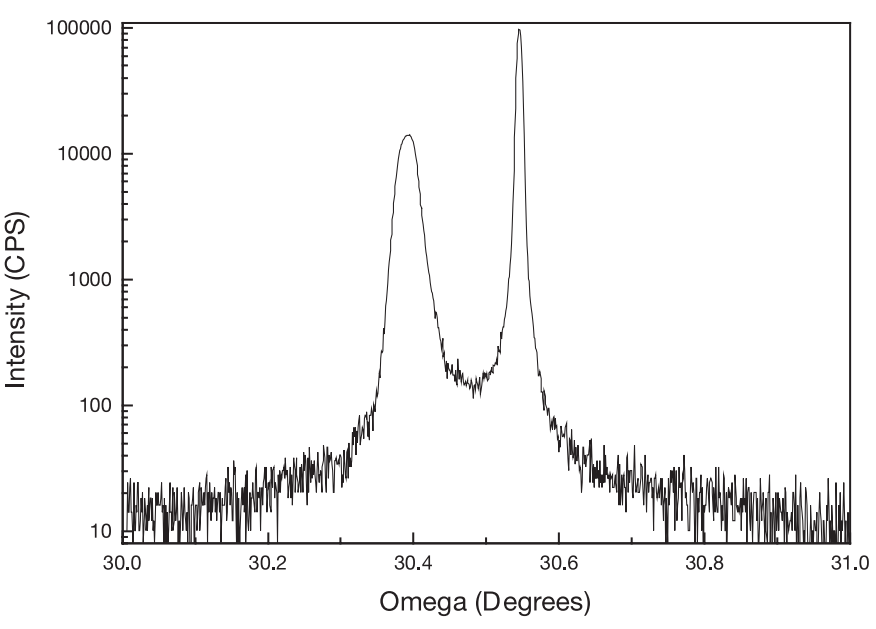

Fig. 8.33. X-ray diffraction pattern of an $400 \mathrm{~nm}$ thick InAsSb layer with $9 \%$ antimony.

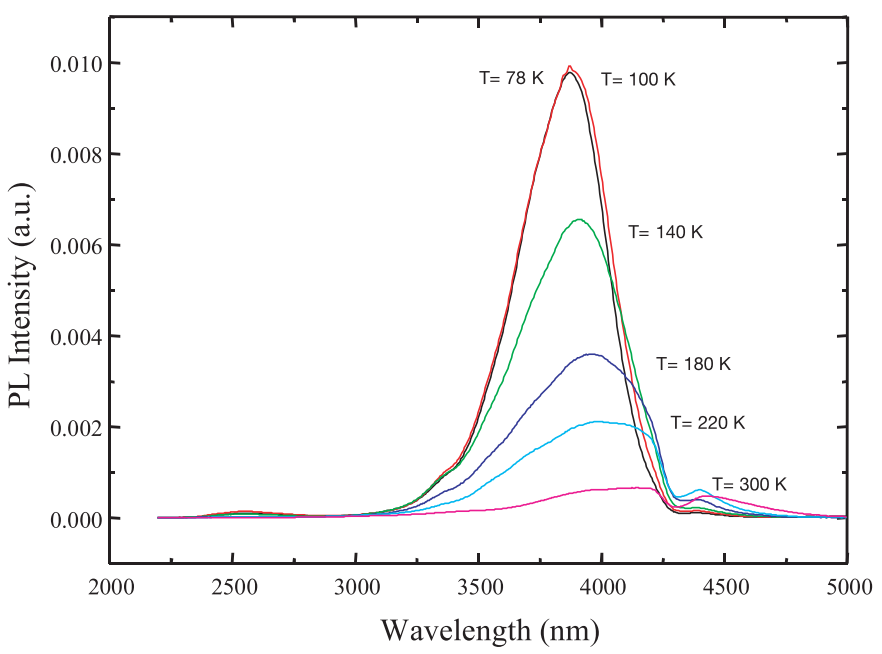

Fig. 8.34. PL spectra for an InAsSb/InAsSb superlattice. 


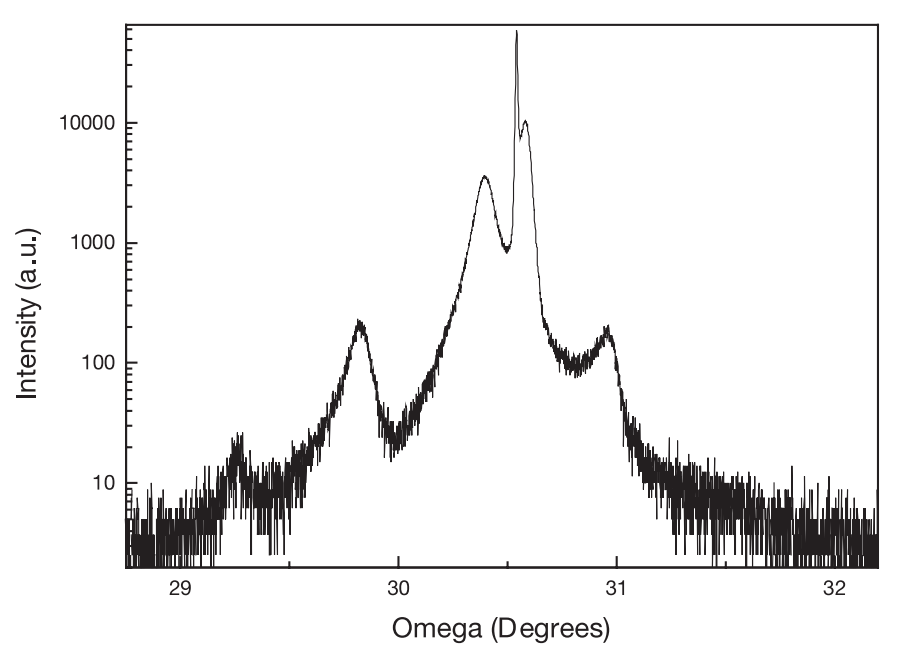

Fig. 8.35. X-ray diffraction spectra for an InAsSb/InAs superlattice.

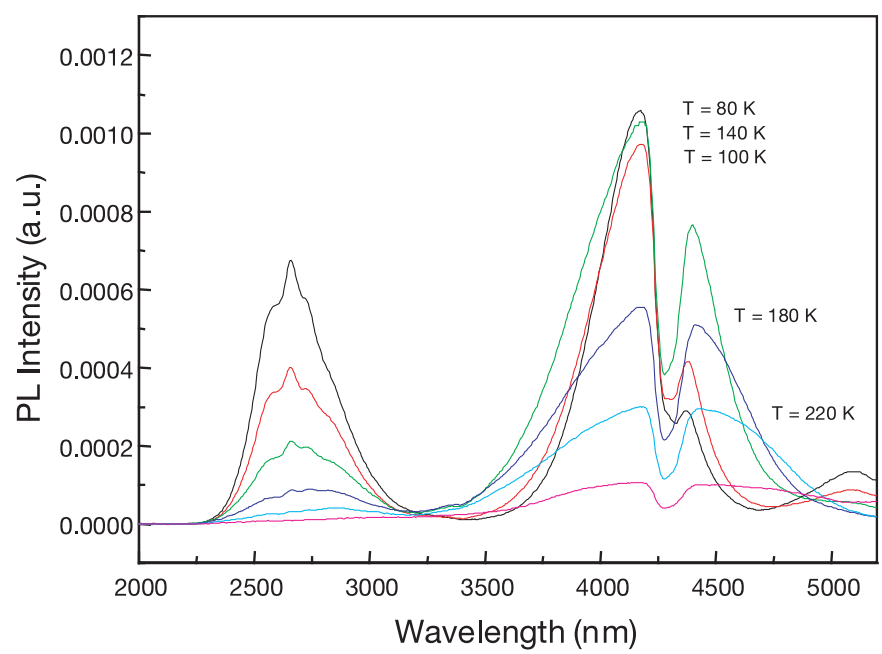

Fig. 8.36. PL spectra for an InAsSb/InAs superlattice. The spectra is distorted at $\sim 4200 \mathrm{~nm}$ as a result of $\mathrm{CO}_{2}$ absorption.

years after [60]. The flexibility of the material to cover a huge infrared range $(2$ to $>50 \mu \mathrm{m})$ and the possibility of a reduced Auger recombination rate [61] drew the attention of many groups. Type-II heterojunctions have been found many applications in electronic devices such as resonant tunneling diodes (RTD) and hot electron transistors. However, perhaps the most important applications have been in optoelectronics. Recently, many significant results have been achieved from type-II modulators [62], detectors $[63,64]$, and laser diodes $[65,66]$. The active layer of photovoltaic and photoconductive type-II detectors are made from superlattices with a type-II band alignment. Similar to a type-I superlattice, the allowed energy states form "mini bands", due to the coupling of electrons and holes in adjacent wells. However, unlike type-I superlattices the bandgap of type-II superlattices can be adjusted from nearly $0.7 \mathrm{eV}$ to virtually zero. The minimum of the mini bands of such superlattice in momentum space are at zero value, and hence the superlattice resembles a direct gap semiconductor. Therefore, knowing the band

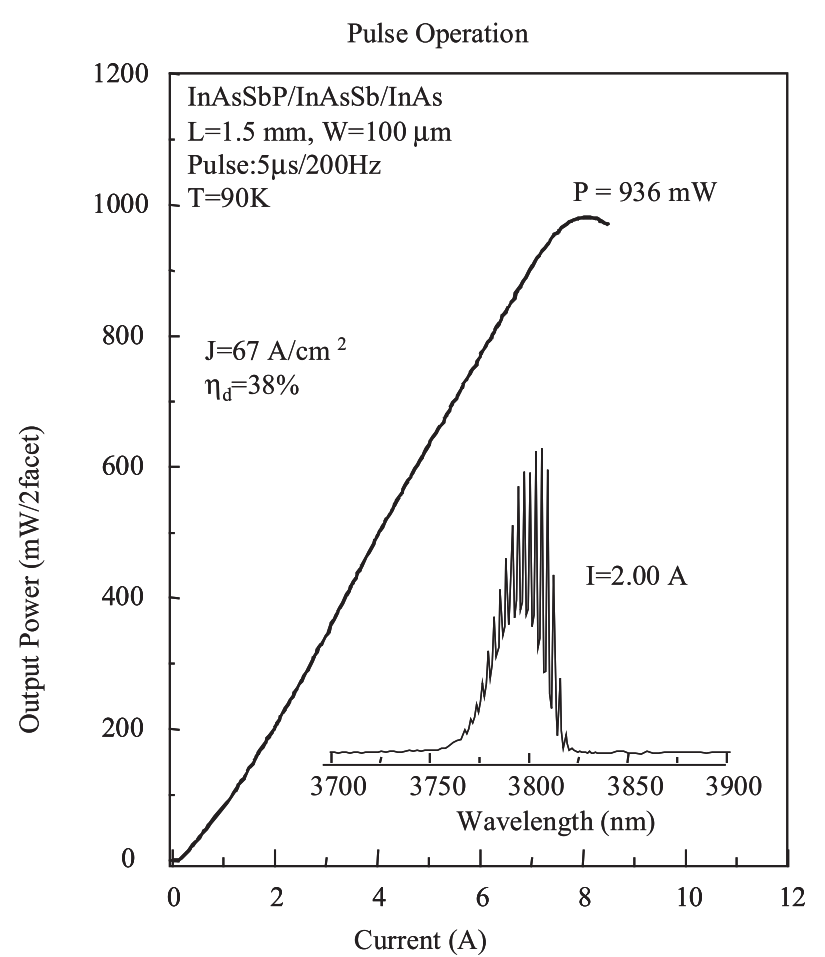

Fig. 8.37. Pulsed output power versus injected current per two facets for an InAs/InAsSb laser at $T=90 \mathrm{~K}$. The emitting spectra, centered around $3.80 \mu \mathrm{m}$, is shown in the inset.

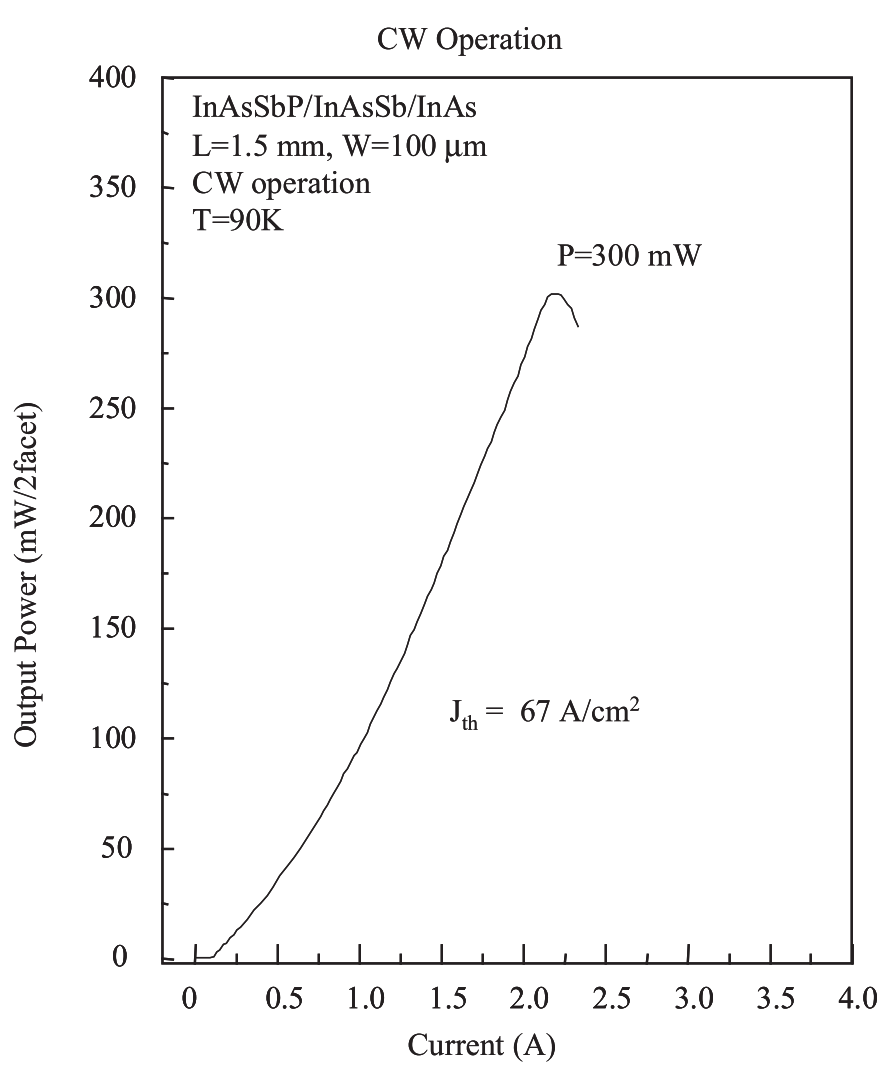

Fig. 8.38. Continuous power for an InAs/InAsSb SLS laser diode with $T=90 \mathrm{~K}$. 


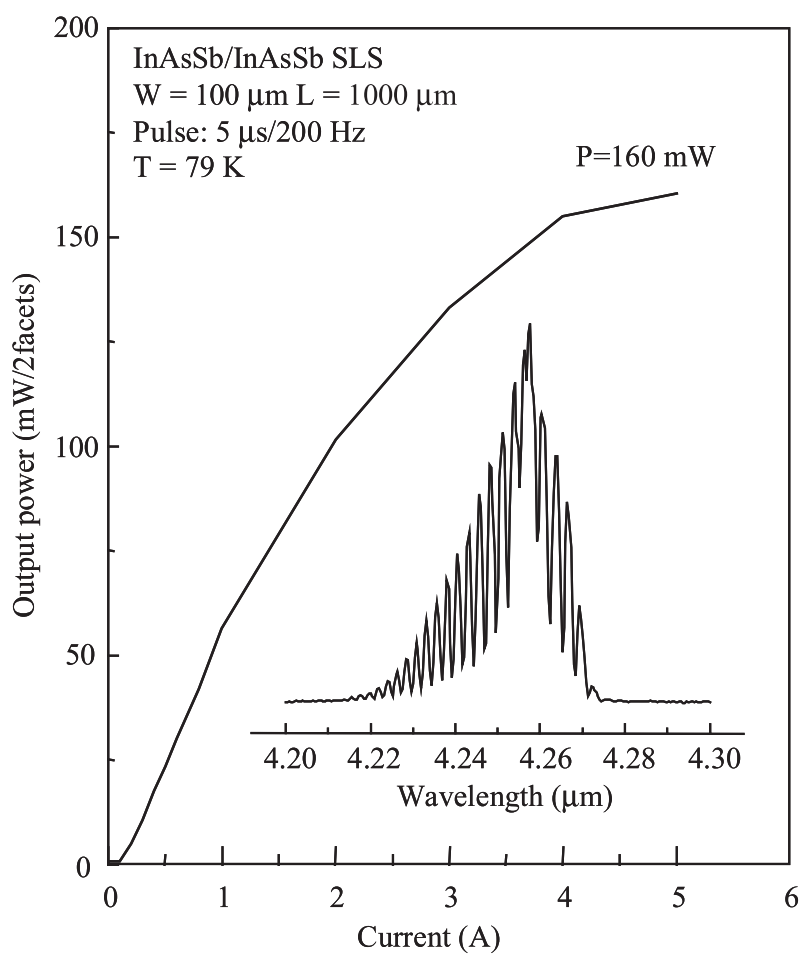

Fig. 8.39. $P I$ curve of a single stripe, 100 period InAsSb/InAsSb laser at $T=80 \mathrm{~K}$. The emission spectrum is shown in the inset.

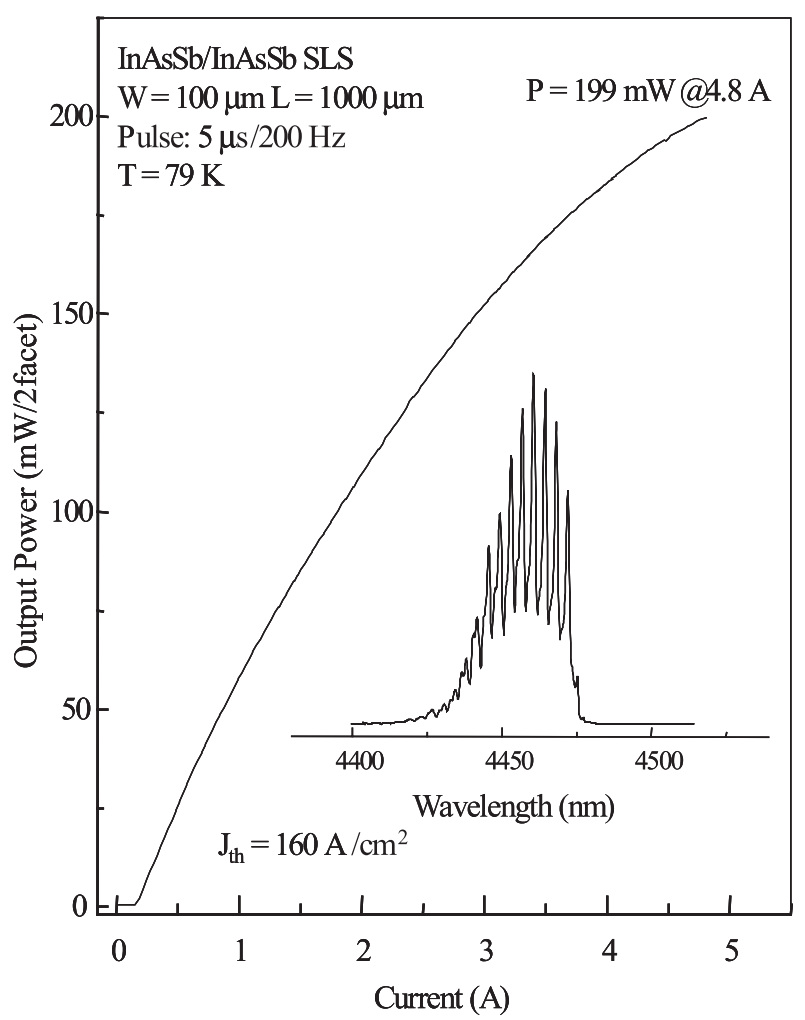

Fig. 8.40. $P I$ curve of a single stripe, 100 period InAsSb/InAsSb laser at $T=80 \mathrm{~K}$. The emission spectrum is shown in the inset.

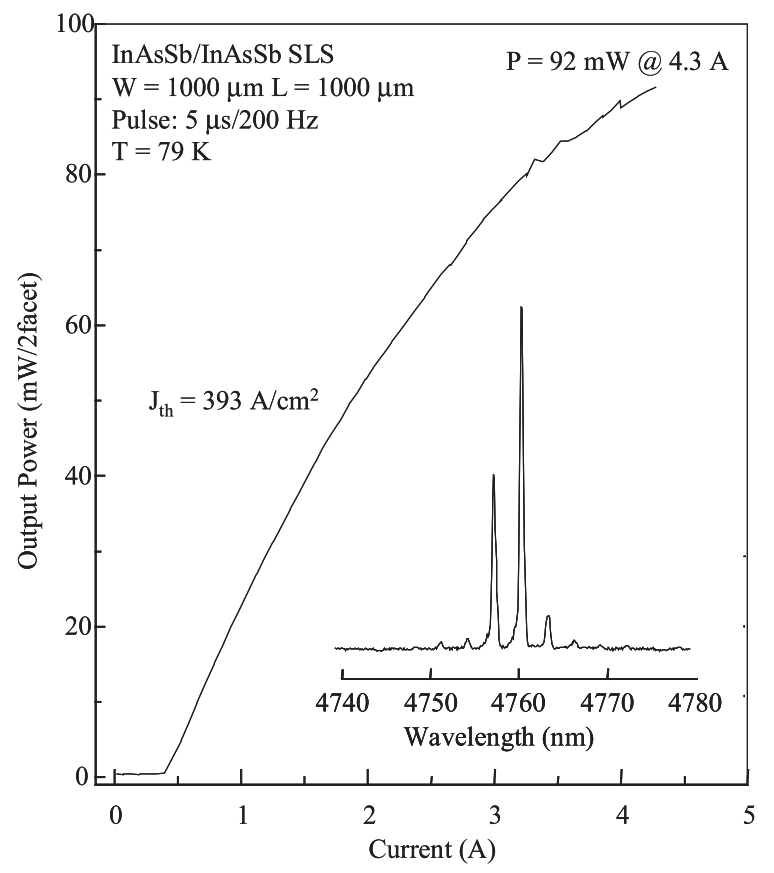

Fig. 8.41. $P I$ curve of a single stripe, 100 period InAsSb/InAsSb laser at $T=79 \mathrm{~K}$. The emission spectrum is shown in the inset.

structure and the optical absorption in type-II superlattices, one can practically use the conventional photovoltaic and photoconductive structures to realize high performance type-II detectors.

InAs/ $\mathrm{Ga}_{x} \mathrm{In}_{1-x} \mathrm{Sb}$ type-II superlattices are very promising system in the long wavelength ranges. InAs, $\mathrm{GaSb}$, and $\mathrm{AlSb}$ and their related compounds form a nearly lattice matched family of semiconductors known as the $6.1 \AA$ family, since the lattice constants of these material are about $a=6.1$ Angstrom. Figure 9.1 shows their energy gaps and lattice constants compared to the other major semiconductors. Table 9.1 shows some of the important properties of the $6.1 \AA$ family.

The numerical modeling of the superlattice energy bands has been carried out by using the Envelope Function Approximation method. Figure 9.1 shows the calculated mini band energy profile of type-II superlattices with equal InAs and GaSb layers as a function of the period of the superlattice. As an example, a $60 \AA$ period will give an emission wavelength around $4 \mu \mathrm{m}$. Figure 9.2 shows that a band engineered type-II superlattice shows Auger resonance at large $k_{\perp}$ values which leads to suppression of Auger recombination.

\subsection{Experimental results for type-II photodetectors}

9.2.1 Uncooled type-II photoconductor in the long wavelength IR (LWIR) range.

\subsubsection{Growth}

The InAs/GaSb type-II superlattices were grown by molecular beam epitaxy (MBE) on semi-insulating GaAs 
Table 9.1. Some of the important properties of InAs, GaSb, and AlSb.

\begin{tabular}{|c|c|c|c|}
\hline $\begin{array}{l}\text { Parameters at } 300 \mathrm{~K} \text { (unless } \\
\text { indicated otherwise) }\end{array}$ & InAs & $\mathrm{GaSb}$ & $\mathrm{AlSb}$ \\
\hline Lattice constant (angstrom) & 6.058 & 6.094 & 6.136 \\
\hline Density $\left(\mathrm{g} / \mathrm{cm}^{3}\right)$ & 5.70 & 6.61 & 4.26 \\
\hline Melting point (K) & 1215 & 985 & 1338 \\
\hline Expansion coefficient & $4.52 \times 10^{-6}$ & $6.2 \times 10^{-6}$ & $4 \times 10^{-6}$ \\
\hline Thermal conductivity $(\mathrm{W} / \mathrm{K} \mathrm{cm})$ & 0.48 & 0.4 & $\sim 0.7$ \\
\hline Energy gap (electron volt) & $\begin{array}{l}0.356 \text { at } 300 \mathrm{~K} \\
0.414 \text { at } 77 \mathrm{~K}\end{array}$ & $\begin{array}{l}0.7 \text { at } 300 \mathrm{~K} \\
\text { - at } 77 \mathrm{~K}\end{array}$ & $\begin{array}{l}1.63 \text { at } 300 \mathrm{~K} \\
1.666 \text { at } 77 \mathrm{~K}\end{array}$ \\
\hline Electron mobility $\left(\mathrm{cm}^{2} / \mathrm{V} \mathrm{s}\right)$ & $\begin{array}{l}20000 \text { at } 300 \mathrm{~K} \\
35000 \text { at } 77 \mathrm{~K}\end{array}$ & $\begin{array}{l}5000 \text { at } 300 \mathrm{~K} \\
\text { - at } 77 \mathrm{~K}\end{array}$ & $\begin{array}{l}200 \text { at } 300 \mathrm{~K} \\
\text { - at } 77 \mathrm{~K}\end{array}$ \\
\hline Hole mobility $\left(\mathrm{cm}^{2} / \mathrm{Vs}\right)$ & $\begin{array}{l}480 \text { at } 300 \mathrm{~K} \\
\text { - at } 77 \mathrm{~K} \\
\end{array}$ & $\begin{array}{l}880 \text { at } 300 \mathrm{~K} \\
2400 \text { at } 77 \mathrm{~K}\end{array}$ & $\begin{array}{l}375 \text { at } 300 \mathrm{~K} \\
\text { - at } 77 \mathrm{~K}\end{array}$ \\
\hline Electron effective mass & $0.023 m_{0}$ & $0.042 m_{0}$ & 0.12 \\
\hline Hole effective mass & $0.42 m_{0}$ & $0.4 m_{0}$ & $0.98 m_{0}$ \\
\hline LO phonon energy at zone center & $29.6 \mathrm{meV}$ & $28.8 \mathrm{meV}$ & $42.1 \mathrm{meV}$ \\
\hline TO phonon energy at zone center & $26.9 \mathrm{meV}$ & $27.7 \mathrm{meV}$ & $39.5 \mathrm{meV}$ \\
\hline Refractive index & $\begin{array}{l}3.42 \text { at } 10 \mu \mathrm{m} \\
3.52 \text { at } 3.7 \mu \mathrm{m} \\
4.56 \text { at } 0.517 \mu \mathrm{m}\end{array}$ & $\begin{array}{l}3.84 \text { at } 10 \mu \mathrm{m} \\
3.79 \text { at } 2 \mu \mathrm{m} \\
3.92 \text { at } 1.55 \mu \mathrm{m}\end{array}$ & $\begin{array}{l}2.995 \text { at } 20 \mu \mathrm{m} \\
2.08 \text { at } 15 \mu \mathrm{m} \\
3.1 \text { at } 10 \mu \\
3.3 \text { at } 2 \mu \mathrm{m}\end{array}$ \\
\hline Dielectric constant (static) & 15.15 & 15.7 & 12.04 \\
\hline
\end{tabular}

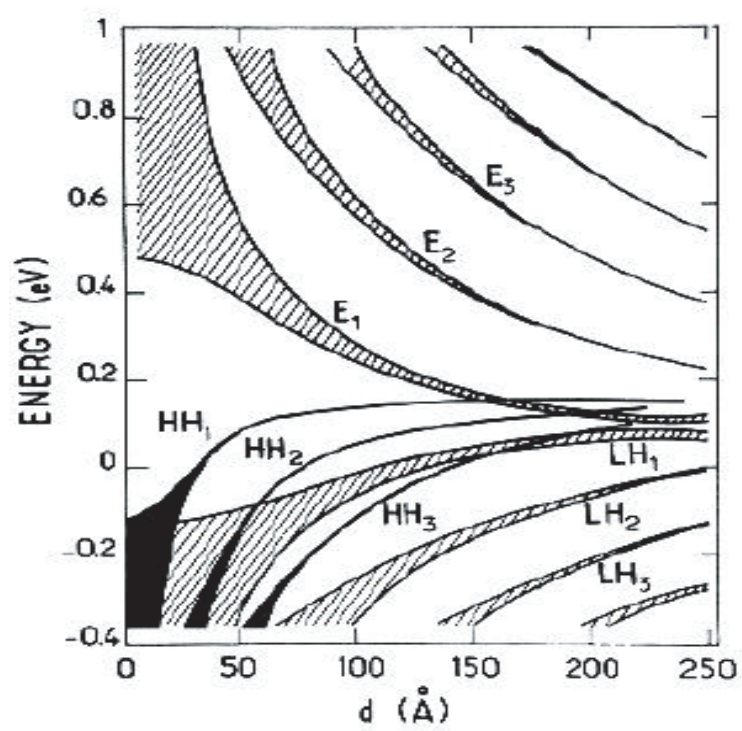

Fig. 9.1. The mini band energy profile of type-II superlattices with equal InAs and GaSb layers versus the period of the superlattice.

substrates [67]. The reactor is an Intevac Modular Gen II MBE machine with uncracked As and $\mathrm{Sb}$, and elemental $\mathrm{Ga}$, In, and Al source material. A $4 \mu \mathrm{m}$ GaSb buffer layer was grown directly on 3" GaAs substrates. The wafer was then broken into $\sim 1 \mathrm{~cm}^{2}$ pieces and indium-mounted to molybdenum blocks. InAs is found to have a very narrow

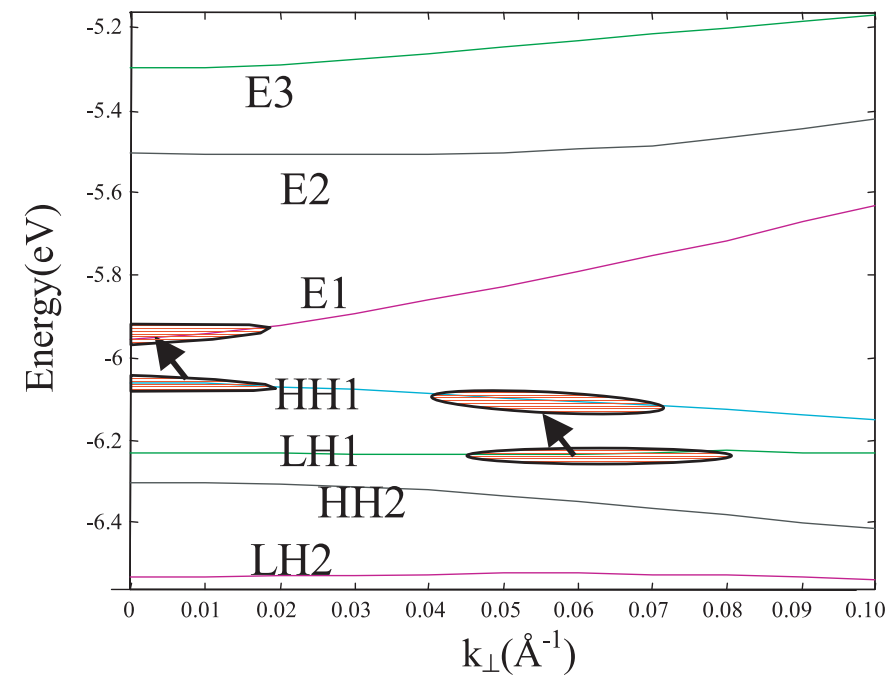

Fig. 9.2. The band structure of a band engineered type-II superlattice shows Auger resonance at large $k_{\perp}$ values which leads to suppression of Auger recombination.

window for planar growth, while high quality GaSb can be grown in a wider range of growth conditions when reflection high energy electron diffraction (RHEED) showed a $1 \times 3$ reconstruction pattern. The optimum growth conditions for InAs layers were found to be: $T=400{ }^{\circ} \mathrm{C}$ according to a pyrometer, a $\mathrm{V}$ to III incorporation rate ratio $\approx 3$, and a growth rate of 0.5 monolayer/s. In this 


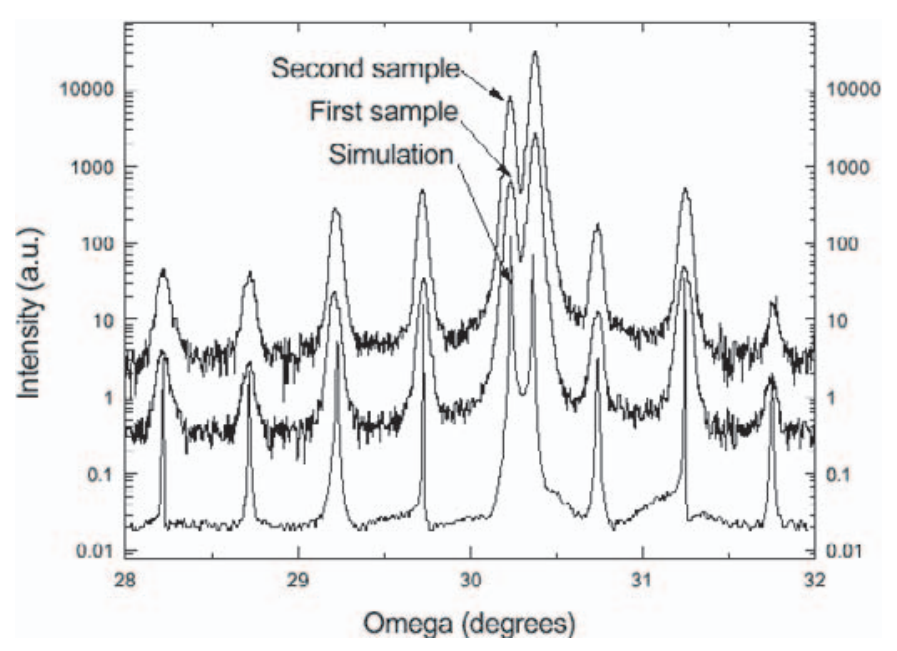

Fig. 9.3. High resolution X-ray diffraction of two samples and the simulation result. Although one of the samples was grown one week after the other, they are almost identical and the simulation is also in good agreement with them.

condition, RHEED showed $2 \times 4$ reconstruction patterns. The pyrometer is calibrated with the temperature of the transition from a $1 \times 5$ to a $1 \times 3$ reconstruction pattern in the GaSb buffer layers. Based on the theoretical modeling and simulation we chose the optimum structure for a room temperature detector at $\lambda=11 \mu \mathrm{m}$. The structure consisted of a $2 \mu \mathrm{m}$ superlattice with $48 \AA$ InAs, $30 \AA$ $\mathrm{GaSb}$, and one monolayer of InSb at the interfaces as it is shown to improve the optical and electrical quality of the superlattice [68]. Finally, the superlattice was capped with a thin $200 \AA$ GaSb layer.

\subsubsection{Characterization}

High resolution X-ray diffraction was used to investigate the structure of the material. X-ray diffraction simulation has also been performed to verify the superlattice structures. Figure 9.3 shows the typical X-ray diffraction of the type-II superlattices and the simulation results. Sharp $\mathrm{X}$-ray peaks up to the 5 th order satellite were observed. This is an indication of high crystal quality and superlattice uniformity in the samples, despite more than $6 \%$ mismatch between the lattice constant of the superlattices and the GaAs substrates. This figure also shows the reproducibility of the growth, since the second sample was grown a week after the first one.

Figure 9.3 shows that the mismatch between the average lattice constant of the SL and the lattice constant of the GaSb buffer layer is about $0.3 \%$. Although high quality SLs with thickness of about $0.5 \mu \mathrm{m}$ can be grown with such mismatch, the mismatch has to be reduced for thicker superlattices. Although the average lattice constant of the superlattice can be adjusted by changing the thickness of the InAs layer, this will also change the bandgap of the SL! Interface modification provides a better method to change the average lattice constant of the superlattices

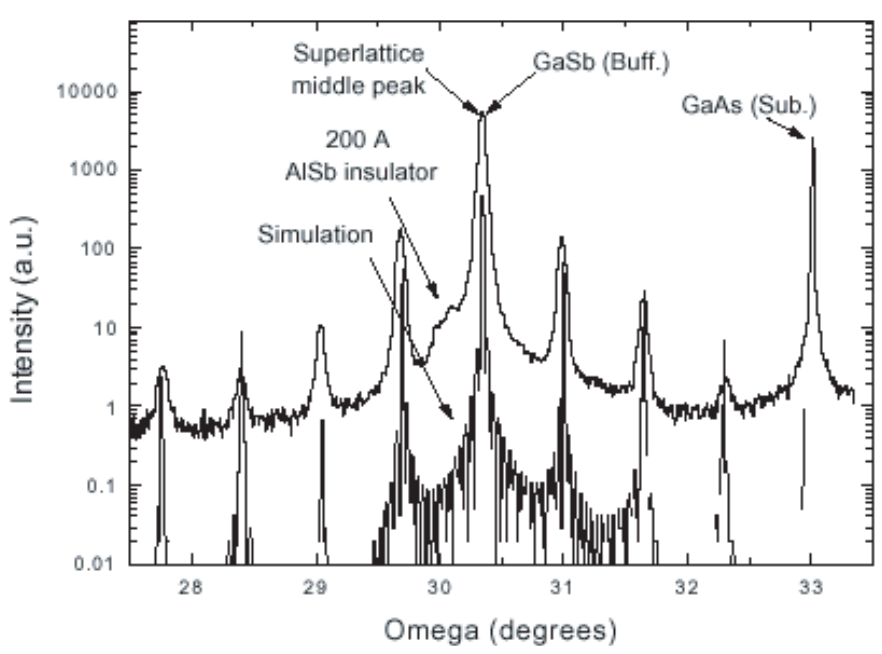

Fig. 9.4. High resolution X-ray diffraction of a superlattice grown on a $200 \AA$ AlSb layer on a GaSb buffer layer. The SL has a lattice mismatch of less than $0.05 \%$ to the GaSb buffer layer.

with a little effect on their bandgap. The interfaces can be either InSb-type or GaAs-type. Despite the fact that these layers are very thin (about 1 monolayer), they have a considerable effect on the average lattice constant of the SL, since the mismatch between $\mathrm{InSb}$ and GaSb is about $+0.6 \%$ and the mismatch between GaAs and GaSb is $-0.6 \%$. Considering the thickness of the interfaces, a precise adjustment of these layers is obviously a difficult task. We managed to routinely grow SLs with very low mismatch to the GaSb buffer layers (see Fig. 9.4) by utilizing two techniques. The atomic beam fluxes Ga, In, Sb, and As were calibrated with dynamic RHEED technique to below $1 \%$ error before each growth. Also, a low growth rate of about 0.5 monolayer per second was used, and therefore the deposition time of the interface was about two seconds which is considerably longer than the actuation time of the shutters in our MBE system $(\sim 0.1 \mathrm{~s})$.

A Mattson Instrument Galaxy 3000 Fourier transform infrared (FTIR) spectrometer was used to obtain the optical characteristics of the superlattices. Figure 9.5 shows the room temperature optical absorption spectra of the superlattice [69]. The effect of the substrate and GaSb buffer layer was removed by measuring the background with a substrate and GaSb buffer layer. The oscillatory features between $0.3 \mathrm{eV}$ to $0.5 \mathrm{eV}$ are due to the interference of internal reflections inside the SL (Fabry Perot oscillation) and the features around $0.175 \mathrm{eV}(\sim 6-7 \mu \mathrm{m})$ are artifacts due to the high atmospheric in this absorption wavelength (mainly water vapor absorption lines).

Since electrons and holes are confined in InAs and GaSb respectively, the Hall measurement technique could not be use on superlattices because of the high sheet density of electrons and holes in the InAs and GaSb layers. The overall Hall coefficient for this material $R_{H}$, and 


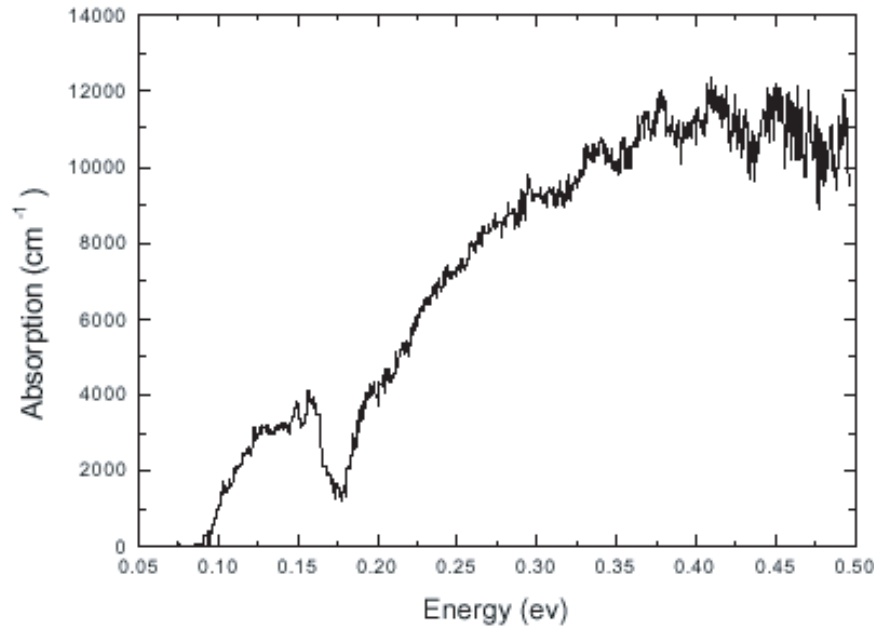

Fig. 9.5. Room temperature absorption spectrum of a $0.5 \mu \mathrm{m}$ thick sample. The absorption at $0.12 \mathrm{eV}$ is comparable to the absorption of $\mathrm{HgCdTe}$ with similar bandgap.

conductance $r$, with two dominant channels of electrons and holes can be approximated as [70]:

$$
\begin{aligned}
& R_{H}= \\
& \frac{\frac{\mu_{p} \sigma_{p}}{1+\mu_{p}^{2} B^{2}}-\frac{\mu_{n} \sigma_{n}}{1+\mu_{n}^{2} B^{2}}}{\left(\frac{\sigma_{p}}{1+\mu_{p}^{2} B^{2}}+\frac{\sigma_{n}}{1+\mu_{n}^{2} B^{2}}\right)^{2}+B^{2}\left(\frac{\mu_{p} \sigma_{p}}{1+\mu_{p}^{2} B^{2}}-\frac{\mu_{n} \sigma_{n}}{1+\mu_{n}^{2} B^{2}}\right)}
\end{aligned}
$$

where $B$ is the magnetic field in the Hall measurement, and $\sigma$ and $\mu$ are the conductivity of each channel and the mobility of carriers. The formula shows clearly that the overall Hall mobility can be much smaller than the real mobility of electrons or holes if the numerator approaches zero. As the transport of electrons is more important for photodetector operation due to its higher mobility, a single quantum well of InAs was grown to examine this property. This provided a simple, fast method for electrical assessment of the interface quality. After the optimization of growth conditions, in-plane room-temperature mobility of electrons in a $75 \AA$ InAs well increased from 5000 to $14000 \mathrm{~cm}^{2} / \mathrm{Vs}$ which is about half the value of bulk InAs.

\subsubsection{Device measurement}

The photoconductor devices were prepared by making Ohmic contacts either with indium annealing or aluminum deposition and etching. The samples were then mounted to a copper heatsink and attached to the cold finger of a liquid nitrogen cryostat equipped with a temperature controller. The spectral photoresponse of the device was measured using FTIR. The samples were illuminated through the front side with normal incidence. The absolute response of the photodetectors was calculated suing a blackbody test set. Figure 9.6 shows the responsivity of the device in the $2-17 \mu \mathrm{m}$ wavelength range at $78 \mathrm{~K}$ and $300 \mathrm{~K}$ with an in-plane electrical field of $5 \mathrm{~V} / \mathrm{cm}$.

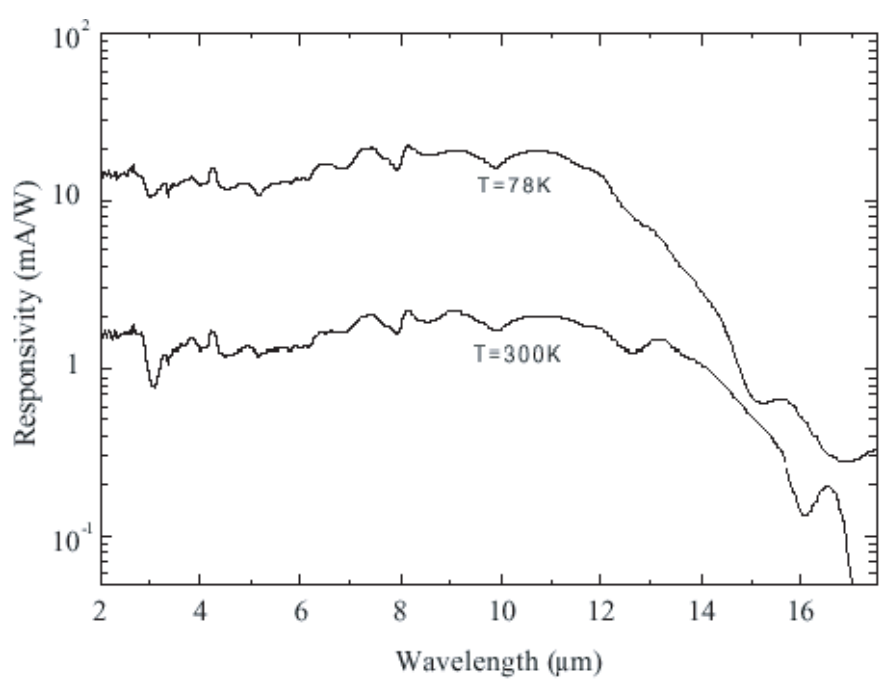

Fig. 9.6. The responsivity spectra of the device at $78 \mathrm{~K}$ and $300 \mathrm{~K}$ with an in-plane electrical field of $5 \mathrm{~V} / \mathrm{cm}$.

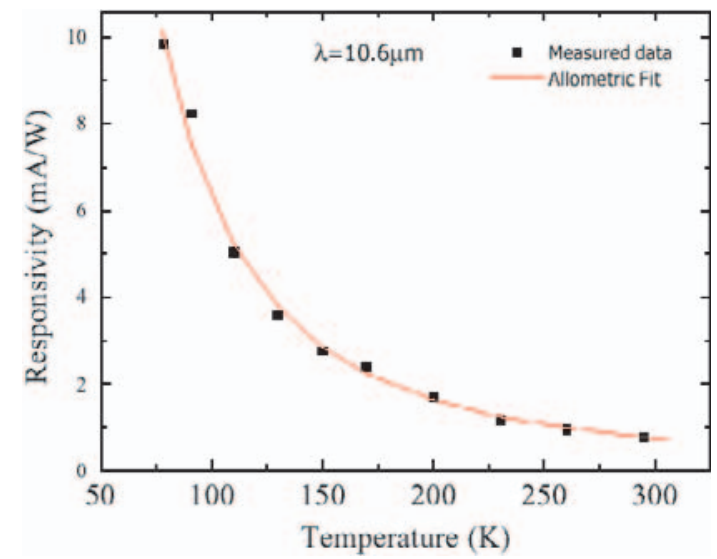

Fig. 9.7. The current responsivity of the device versus temperature at $\lambda=10.6 \mu \mathrm{m}$ at constant voltage bias. The line is an Allometric fit $\left(A T^{B}\right)$ to the measured data which shows $B \sim-2$.

To assess the temperature dependence, the current responsivity of the device was measured at $\lambda=10.6 \mu \mathrm{m}$ wavelength from $78 \mathrm{~K}$ to room temperature at a constant electrical field. The result is shown in Figure 9.7. As shown in the figure, an allometric function (proportional to $T^{-1.93}$ ) fits well to the measured data. Usually the responsivity of the narrow gap materials is an exponential function of temperature at higher temperature where Auger recombination dominates the recombination mechanism. However, it has been showed theoretically by Zegrya et al. [71] that the Auger recombination rate is a power function of the temperature (proportional to $T^{2}$ ) in type-II heterostructures compared to the exponential function in the bulk semiconductors. Since the current responsivity is proportional to the carrier lifetime which is dictated by Auger recombination rate, this power dependency of the responsivity indicates a good agreement with the theoretical predictions. 


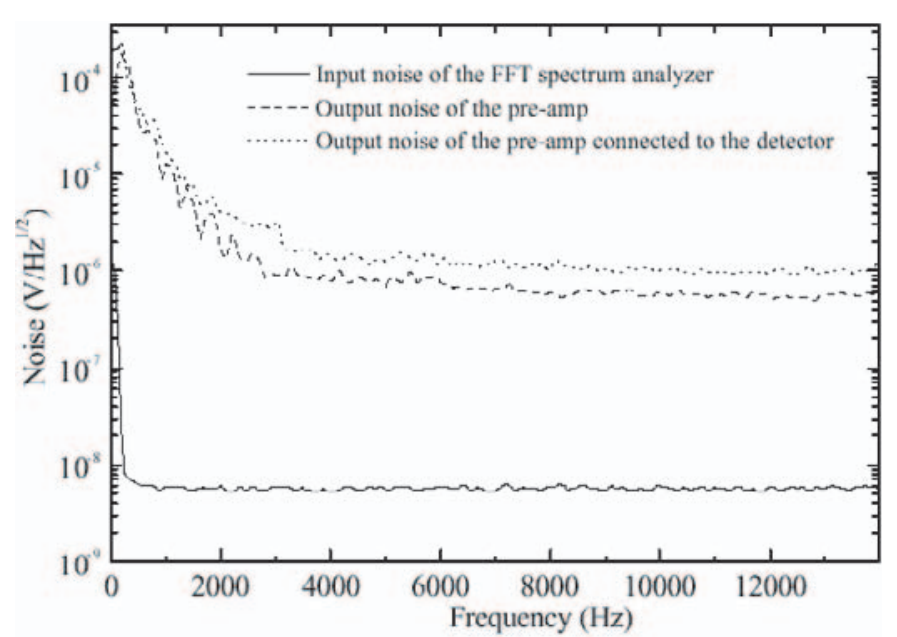

Fig. 9.8. The measured input noise spectrum of the fast Fourier transform (FFT) spectrum analyzer, the output noise spectra of the pre-amplifier with a shorted input and the output noise spectra of the pre-amplifier when it is connected to the detector at $300 \mathrm{~K}$.

The effective lifetime of the carriers extracted from the responsivity and Hall measurements on a $t=0.5 \mu \mathrm{m}$ thick superlattice is $\tau_{\text {ef }}=26.8 \mathrm{~ns}$, which is about an order of magnitude longer than the carrier lifetime in $\mathrm{HgCdTe}$ photoconductors with similar bandgap and carrier concentration at room temperature [72]. Since Auger recombination is the dominant recombination mechanism at room temperature, we believe that the enhancement of carrier lifetime is due to the suppression of Auger recombination in the type-II superlattice.

The noise was measured with a fast Fourier transform (FFT) spectrum analyzer (Stanford Research System SR $760)$ and a low noise, wide band pre-amplifier with $54 \mathrm{~dB}$ voltage gain (EG\&G PA-100). Figure 9.8 shows the input noise spectrum of the FFT analyzer, the output noise spectrum of the shorted pre-amplifier, and the output noise of the pre-amplifier, when it was connected to the detector. The detector was biased by the pre-amplifier at $V_{b}=5$ volts.

The mean-square noise of the detector can be modeled as:

$$
V_{n}^{2}=V_{J}^{2}+V_{1 / f}^{2}+V_{G R}^{2}
$$

where $V_{n}$ is the overall noise of the detector, $V_{J}$ is the Johnson-Nyquist noise, $V_{1 / f}$ is the $1 / f$ noise and $V_{G R}$ is the generation-recombination noise. The value of the Johnson noise can be calculated as

$$
V_{J}^{2}=4 k T R \Delta f
$$

where $k$ is the Boltzmann constant, $T$ is the temperature and $R=76 \mathrm{ohms}$ is the resistance of the device. The value of Johnson noise for the device at room temperature is $1.12 \mathrm{nV} / \mathrm{Hz}^{1 / 2}$. The $1 / f$ noise can be approximated with:

$$
V_{1 / f}^{2}=V_{G R}^{2} \frac{f_{1 / f}}{f}
$$

where $f_{1 / f}$ is a constant which depends on the sample, and $f$ is the frequency. This shows that at high enough frequencies, $1 / f$ noise can be negligible compared to the other two types of noise. Then the value of the generation-recombination noise can be extracted from the total noise of the device in this range. The noise equivalent circuit model [73] was used to extract the noise of the photodetector as $V_{n}=1.7 \mathrm{nV} / \mathrm{Hz}^{1 / 2}$ above $10 \mathrm{kHz}$. From the above equations, the value of the generation-recombination noise can be calculated as $V_{G R}=1.28 \mathrm{nV} / \mathrm{Hz}^{1 / 2}$. The generation-recombination noise can be approximated as [74]

$$
V_{G R}=\frac{2 V_{b}}{(l w t)^{1 / 2}} \frac{1+b}{b n+p}\left(\frac{n p}{n+p} \frac{\tau_{\Delta} f}{1+\omega^{2} \tau^{2}}\right)
$$

where $w=4 \mathrm{~mm}$ is the detector width, $\omega$ is the angular frequency and $\omega \tau \ll 1$ in this experiment, and $b$ is the ratio of $m_{e}$ to $m_{p}$. From the $V G R$ equation, the value of the carrier lifetime can be calculated as $t=17 \mathrm{~ns}$ which is close to the value of the lifetime extracted from the optical response measurement.

The response time of the type-II uncooled detectors was measured using a room temperature quantum cascade laser, developed at CQD [75], as a narrowband and high speed infrared source in our measurement. The quantum cascade laser was uncooled and operated at $\lambda=8.5 \mu \mathrm{m}$ with a negligible time delay. With the time response measurement setup [76], it shows that the detector has a fall time below $40 \mathrm{~ns}$.

The performance of infrared detectors is usually compared based on their detectivity which is an indication of their sensitivity. Knowing the responsivity and the noise of the devices, we could calculate their detectivity. The uncooled devices show a detectivity of about $1.3 \times 10^{8} \mathrm{~cm} \mathrm{~Hz}^{1 / 2} / \mathrm{W}$ at $\lambda=11 \mu \mathrm{m}$ which is higher than the detectivity of commercially available $\mathrm{HgCdTe}$ at similar wavelengths and temperature [77]. Unlike $\mathrm{HgCdTe}$, these type-II superlattices are grown on GaAs substrates, and hence highly uniform material can be grown on three and five inch wafers readily. In comparison to the thermal detectors, such as microbolometers, type-II superlattices have similar detectivity, but are showing at least five orders of magnitude faster response [78].

\subsubsection{Realization of type-II photoconductors in the very long wavelength IR (VLWIR) range}

High performance infrared detectors with cutoff wavelength above $16 \mu \mathrm{m}$ are highly needed for space based applications such as deep-space astronomy and pollution monitoring. Currently available detectors with high quantum efficiency in this wavelength range are $\mathrm{HgCdTe}$ and extrinsic silicon detectors. However, due to the high nonuniformity of $\mathrm{HgCdTe}$, detector arrays with acceptable uniformity can only be realized with extrinsic silicon detectors at long wavelength. Although high detectivity and good uniformity have been achieved with this type of detectors, they have to be cooled below $10 \mathrm{~K}$. Consequently, 
a three-stage cryo-cooler is required which is heavy, bulky, and has a short lifetime. These drawbacks are especially important for space applications since they significantly increase the launch cost. Theoretical [78] calculation and our experimental results [79] show that $\operatorname{InAs} / \mathrm{Ga}_{1-x} \operatorname{In}_{x} \mathrm{Sb}$ type-II superlattices have similar absorption coefficient to $\mathrm{HgCdTe}$, and therefore detectors with very high quantum efficiencies are possible. However, unlike MCT the uniformity of the growth is not an issue in this material system due to the strong bonding of III-V compound semiconductors.

\subsubsection{Growth}

The structures were grown on semi-insulating GaAs substrates. First, GaSb buffer layers with a thickness of about $2 \mu \mathrm{m}$ were grown directly on the GaAs substrates. The growth was optimized for low background doping levels $\left(\sim 5 \times 10^{15} \mathrm{~cm}^{-3}\right)$, and high surface smoothness $(\sim 4 \AA$ rms roughness) in this layer. The superlattices consist of InAs and GaSb layers with one monolayer of InSb at both interfaces and were grown directly on GaSb buffer layers. The growth was terminated by a $100 \AA$ GaSb cap layer.

\subsubsection{Device measurement}

Spectral photoresponse was measured using a FTIR spectrometer system. The samples were illuminated through the front side at normal incidence. Absolute response of the photodetectors was calculated using a blackbody setup. Figure 9.9 shows the responsivity of the device in 3-25 $\mu \mathrm{m}$ wavelength range at $80 \mathrm{~K}$ with an in-plane electrical field of $20 \mathrm{~V} / \mathrm{cm}$. Figure 9.10 shows the spectral responsivity of three devices based on three different superlattice structures covering a $12-25 \mu \mathrm{m}$ range.

One of the most important issues in the detectors with long cutoff wavelength is the uniformity of the material, which is a crucial requirement for high resolution focal plane arrays (PFA) where the device area can be several square centimeters. Figure 9.11 shows the relative spectral response of three samples grown at the center, middle, and the edge of a three inch block simultaneously. The $10 \%$ cutoff energy varied only about $5 \mathrm{meV}$ from the center to the edge, showing excellent uniformity over a very large area $[80]$.

\subsubsection{Uncooled type-II photodiodes in the long wavelength IR (LWIR) range}

We demonstrated high performance uncooled photoconductors in the long wavelength range based on type-II superlattices previously, however, these devices are more suitable for single-element detectors and cannot be easily used for two-dimensional (2D) FPA. Unlike a photoconductor, the current of a photodiode flows perpendicular to the surface, and hence it is scaled by the area of the device. Moreover, photodiodes can operate even at zero bias

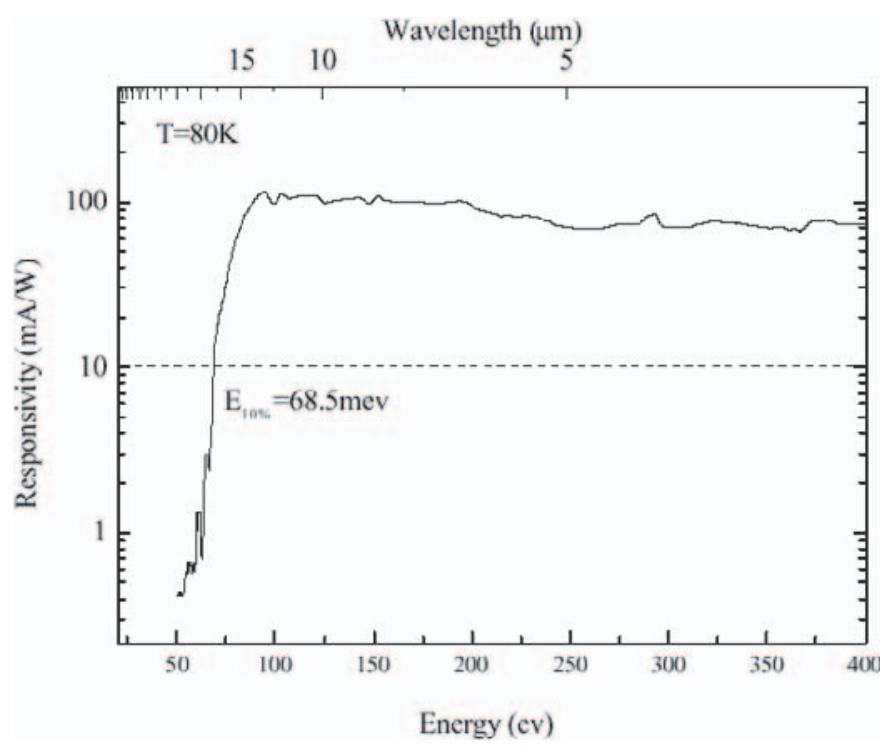

Fig. 9.9. The spectral response of the device.

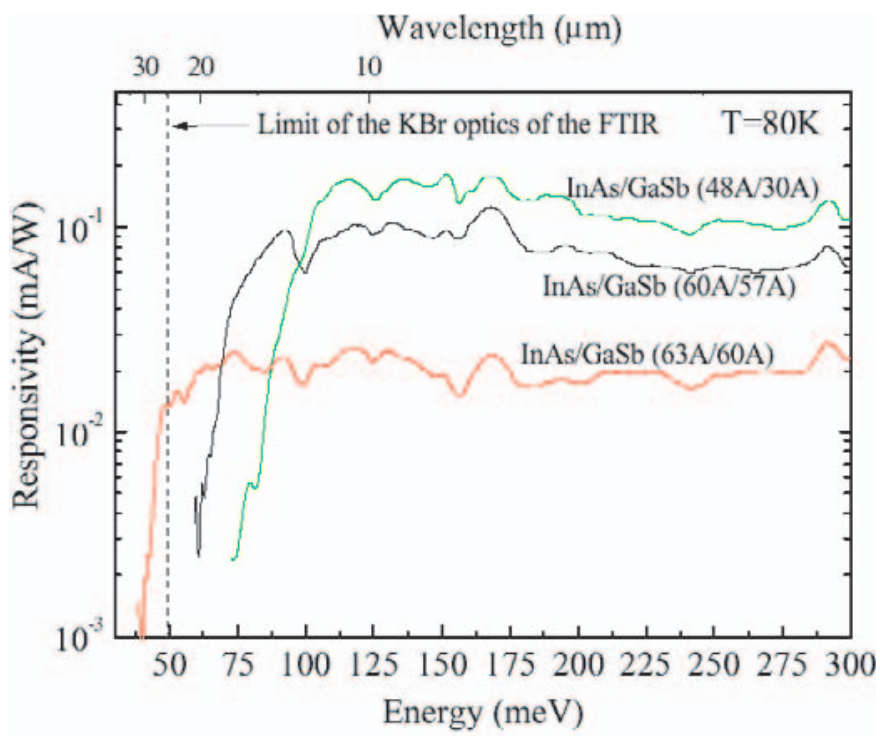

Fig. 9.10. The spectral responsivity of three superlattices with cutoff wavelengths from 12 to $25 \mu \mathrm{m}$ at $80 \mathrm{~K}$.

which not only reduces the bias and heat dissipation requirements significantly, but also eliminates the $1 / f$ noise. This type of noise increases inversely with frequency, and can be the dominant source of the total noise for low frequency applications such as IR imaging systems.

\subsubsection{Growth}

The structures were grown using cracked As and Sb sources. The cracking zone temperature for both cells was $900{ }^{\circ} \mathrm{C}$. The deposition rates of the material were calibrated with dynamic RHEED oscillation to within $1 \%$. First, $1 \mu \mathrm{m}$ of GaSb contact layer doped with Be $\left(N_{A}=1 \times 10^{18} \mathrm{~cm}^{-3}\right)$ was grown. Then a stack of five devices was grown, each of which consisted of 


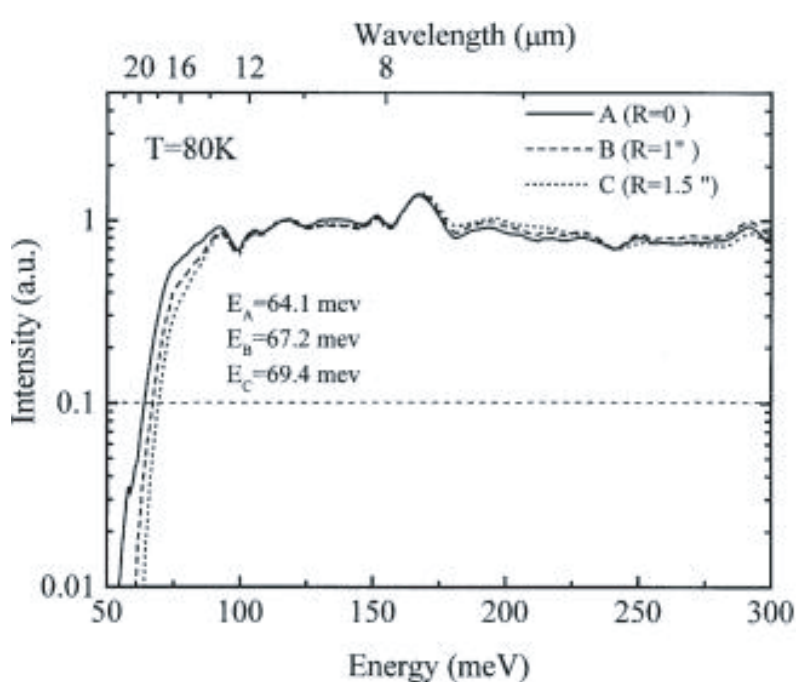

Fig. 9.11. Relative spectral response of three samples from the center, middle, and edge of a 3-in.-diam wafer block.

20 periods of $p$-type InAs/GaSb: Be $(39 \AA / 40 \AA), 20$ periods of nominally undoped InAs/GaSb $(39 \AA / 40 \AA)$, and 20 periods of $n$-type InAs:Si/GaSb (39 $\AA / 40 \AA)$ superlattices. GaSb layers in this superlattice had a graded doping from $1 \times 10^{18} \mathrm{~cm}^{-3}$ to $2 \times 10^{17} \mathrm{~cm}^{-3}$. In the $n$ type superlattice, InAs layers were doped with $\mathrm{Si}\left(N_{D}=\right.$ $\left.2 \times 10^{18} \mathrm{~cm}^{-3}\right)$. The shutter sequences were designed such that both interfaces were InSb type. Finally, the growth was capped with a $0.01 \mu \mathrm{m}$ InAs: Si $\left(N_{D}=1 \times 10^{18} \mathrm{~cm}^{-3}\right)$. The growth temperature was about $520{ }^{\circ} \mathrm{C}$ for the $\mathrm{GaSb}$, and $395{ }^{\circ} \mathrm{C}$ for the superlattices according to a pyrometer calibrated with the surface reconstruction transition temperatures of $\mathrm{GaSb}\left(\sim 390^{\circ} \mathrm{C}\right)$ and $\operatorname{InSb}\left(\sim 380^{\circ} \mathrm{C}\right)$.

\subsubsection{Device measurement}

Absolute spectral responsivity was calculated from the measured spectral response of the devices, using a FTIR spectroscopy system, and the device's photoresponse to a calibrated blackbody setup. The peak responsivity was $R_{i}=0.14 \mathrm{~A} / \mathrm{W}$ at $\lambda=7 \mu \mathrm{m}$ leading to a Johnson noise limited detectivity $D^{*}=1.2 \times 10^{8} \mathrm{~cm} \mathrm{~Hz}^{1 / 2} / \mathrm{W}$ at room temperature. Figure 9.12 shows the detectivity of a device versus IR wavelength and energy.

The operation of type-II photodiodes under zero bias ensures that the main noise component is the thermal (Johnson) noise and that $1 / f$ noise is eliminated. Our experimental measurements indicate that even under a considerable reverse bias, type-II detectors do not have a high frequency $1 / f$ noise. Figure 9.13 shows the frequency spectrum of the amplified output of a detector. The device was under a -0.2 volt bias and illuminated by the chopped IR radiation of a blackbody. The chopper frequency was $f_{0}=396 \mathrm{~Hz}$, blackbody temperature and aperture diameter were $T_{B B}=800 \mathrm{~K}$ and $D_{B B}=2.54 \mathrm{~cm}$, and the detector was located $d=15 \mathrm{~cm}$ apart from the blackbody aperture. Although the measurement includes the noise of the pre-amplifier (Analog Device AD797) and the FFT

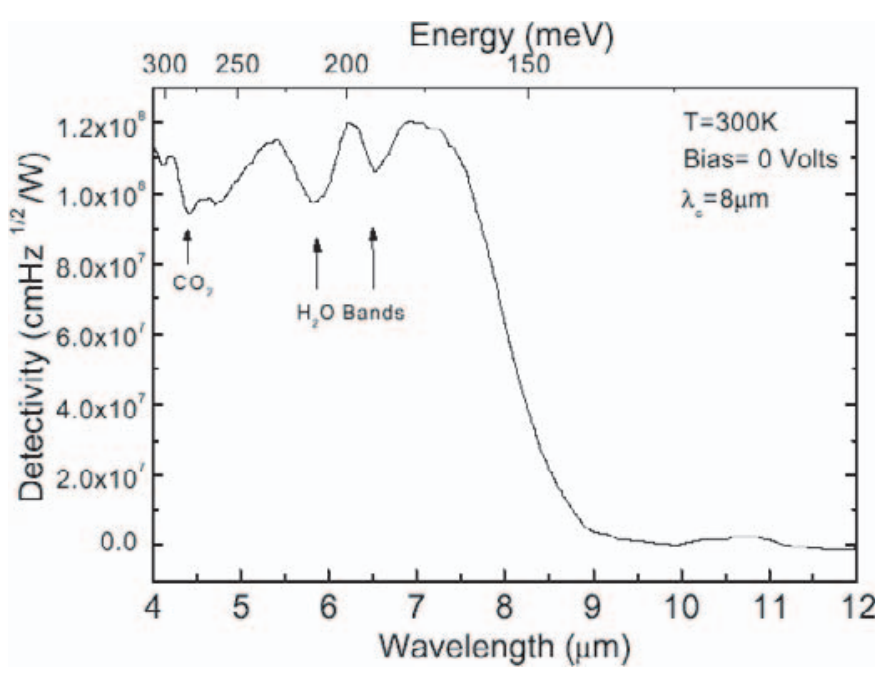

Fig. 9.12. The detectivity of a device versus the wavelength and energy of the IR radiation. Features at $4.3 \mu \mathrm{m}$ and $6-$ $7 \mu \mathrm{m}$ are due to the $\mathrm{CO}_{2}$ and water vapor absorption during the measurement.

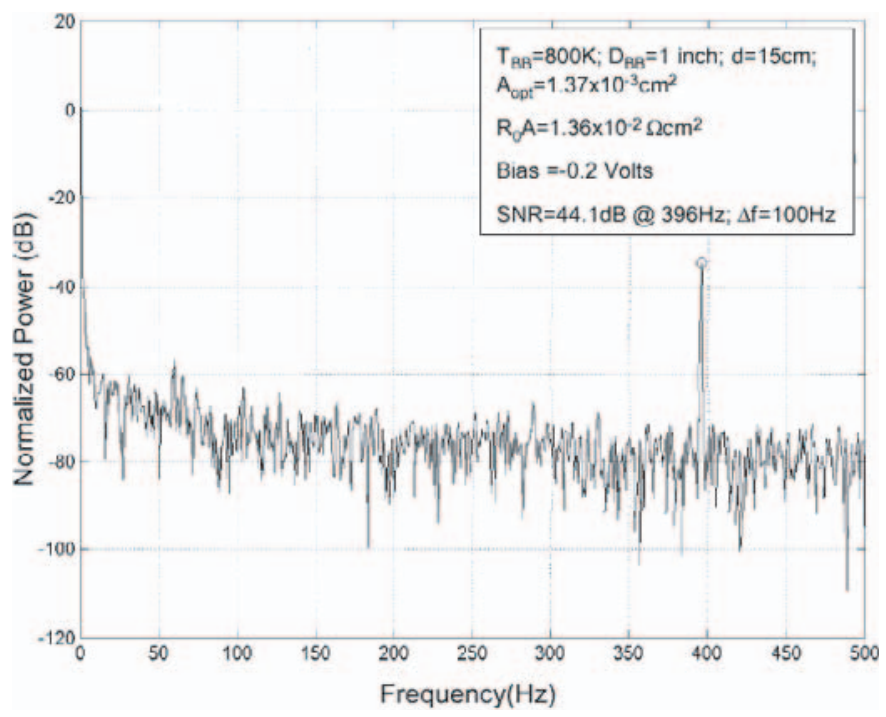

Fig. 9.13. Frequency spectrum of the amplified output of a detector illuminated by a chopped IR radiation of a blackbody. The detector was biased at $-0.2 \mathrm{~V}$ and was located $d=15 \mathrm{~cm}$ apart from the blackbody aperture.

spectrum analyzer (Stanford Research System SR 760), the knee of the $1 / f$ noise is below $\sim 100 \mathrm{~Hz}$. Under the given parameters of the inset of Figure 9.13, the signal to noise ratio (SNR) was more than $44 \mathrm{~dB}$ with a bandwidth of $\Delta f=100 \mathrm{~Hz}$ around $f_{0}=396 \mathrm{~Hz}$.

A quantum cascade laser (QCL), operating at room temperature [81], was used as a high-speed source of IR radiation at $\lambda=5 \mu \mathrm{m}$ to study the response time of the uncooled type-II devices. Figure 9.14 shows the current of the QCL as well as the output of the pre-amplifier versus time. The inset shows the schematic diagram of the measurement setup. The laser threshold current marks the current above which the laser starts emitting. Considering the fall time of the pre-amplifier ( $\sim 50 \mathrm{~ns})$ and the fall time 


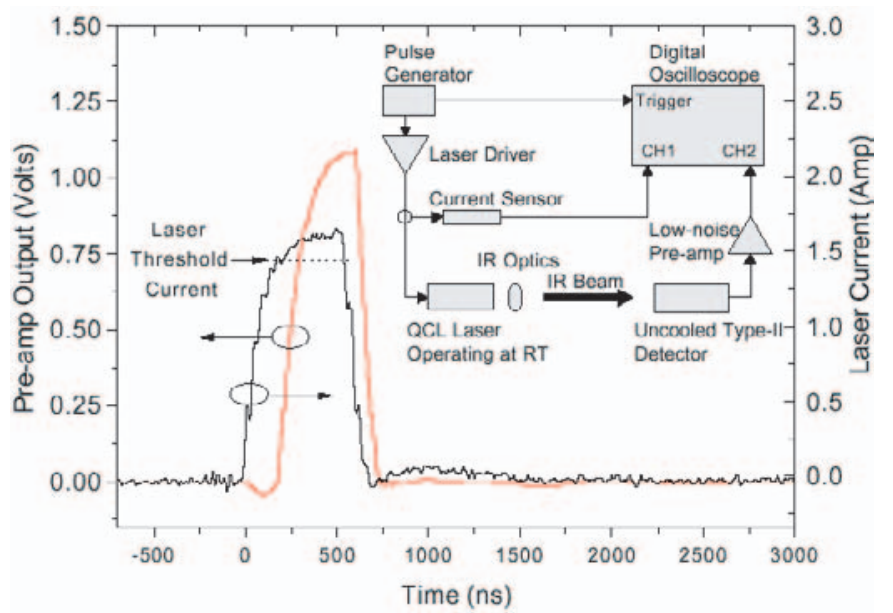

Fig. 9.14. The current of the QCL laser (right axis) and the amplified output of the detector (left axis) versus time. Inset shows the measurement setup. Note that the laser emits when current is above the threshold level.

of the output signal of the pre-amplifier ( $110 \mathrm{~ns})$, the detector response time was about $\left(110^{2}-50^{2}\right)^{1 / 2} \sim 100 \mathrm{~ns}$ using the sum-of-squares approach.

\subsubsection{Realization of type-II photodiodes in the very long wavelength IR (VLWIR) range}

The previous experimental results suggest that type-II superlattice have superior uniformity compared with the available interband detectors in the VLWIR range. However, realizing that the material quality cannot be enhanced on a lattice mismatch substrate, we started the work on GaSb substrates. Photovoltaic devices were the natural choice due to the high conduction of the undoped substrate as well as the benefits of the photovoltaic devices for imaging applications.

\subsubsection{Growth}

The material is grown with $\mathrm{As}$ and $\mathrm{Sb}$ valved cracker sources on $p$-type GaSb substrates [82]. The photodiode structures were grown at $395{ }^{\circ} \mathrm{C}$ according to a calibrated pyrometer. First, a $1 \mu \mathrm{m}$ GaSb buffer/contact layer doped with $\operatorname{Be}\left(p \sim 1 \times 10^{18} \mathrm{~cm}^{-3}\right)$ was deposited. Next a $0.5 \mu \mathrm{m}$ thick InAs/GaSb: Be $\left(p \sim 1 \times 10^{18} \mathrm{~cm}^{-3}\right.$ to $3 \times 10^{17} \mathrm{~cm}^{-3}$ ) superlattice was grown followed by a $2 \mu \mathrm{m}$ thick nominally undoped superlattice. Finally, a $0.5 \mu \mathrm{m}$ thick InAs:Si/GaSb $\left(n \sim 1 \times 10^{18} \mathrm{~cm}^{-3}\right)$ superlattice was grown and capped with a $100 \AA$ thick InAs:Si $\left(n \sim 2 \times 10^{18} \mathrm{~cm}^{-3}\right)$ top contact layer. The growth rate was 0.5 monolayer/s for InAs layers and 0.8 monolayer/s for GaSb layers. The V/III beam-equivalent pressure (BEP) ratio was about 4 for InAs layers and about 1.2 for GaSb layers. The cracker temperature for $\mathrm{As}$ and $\mathrm{Sb}$ cells was $800^{\circ} \mathrm{C}$. The selected thickness of InAs and GaSb layers were determined for specific cutoff wavelengths using a four-band superlattice k.p model. For devices with

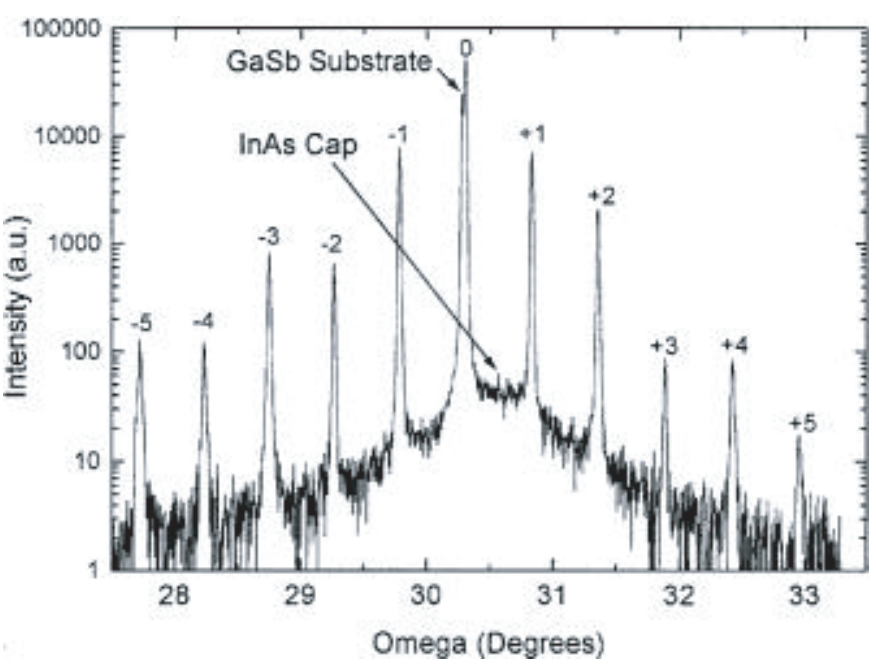

Fig. 9.15. High resolution X-ray diffraction of an InAs/GaSb (54 $\AA / 40 \AA)$ superlattice grown on a GaSb substrate. FWHM of the satellite peaks is below $60 \mathrm{arcs}$, and the mismatch to the substrate is below $0.06 \%$.

nearly a cutoff wavelength of $16 \mu \mathrm{m}$, the thickness of InAs layers was $54 \AA$ and the thickness of the GaSb layers was $40 \AA$.

\subsubsection{Characterization}

Structural quality of the epitaxial layers was assessed using high resolution X-ray diffraction. Figure 9.15 shows the typical X-ray diffraction pattern of the photodiode structures. The mismatch between the average lattice constant of the superlattice and the GaSb substrate was below $0.06 \%$, while the full width at half maximum (FWHM) of the satellite peaks was below 50 arcs for the grown devices.

The surface morphology of the samples was also studied with AFM. Figure 9.16 shows the gray-scale surface morphology of a sample. Wide atomic steps are visible which indicates excellent surface smoothness. Samples with a root-mean-square surface roughness below $4 \AA$ over a $20 \mu \mathrm{m} \times 20 \mu \mathrm{m}$ area can be grown routinely, which is among the best reported values for this material system.

\subsubsection{Device measurement}

The samples were then processed into $400 \mu \mathrm{m} \times 400 \mu \mathrm{m}$ mesas using standard lithography and wet etching. $\mathrm{Ti} / \mathrm{Au}$ contacts were defined for top and bottom contacts with metal evaporation and liftoff techniques. The samples were then indium bonded to a copper heatsink and attached to the cold finger of a liquid nitrogen cryostat with KRS-5 windows.

Figure 9.17 shows the measured and modeled current densities versus the applied bias for devices with cutoff wavelength of $\lambda_{c}=16 \mu \mathrm{m}$. The calculated current density, which consists of tunneling, generation-recombination, 


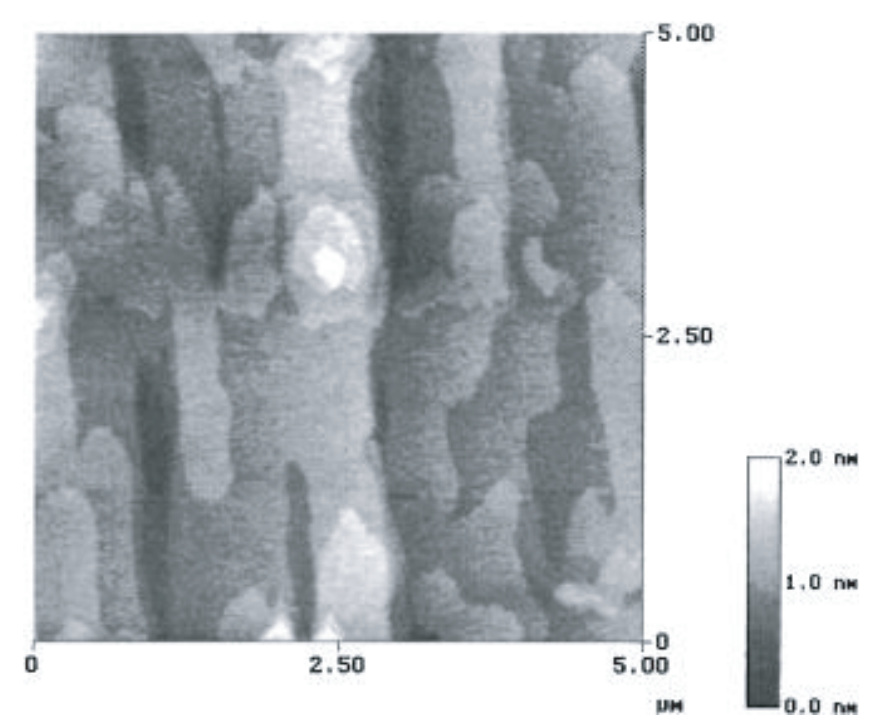

Fig. 9.16. AFM image of the surface of the device. Atomic steps with several microns width are clearly visible.

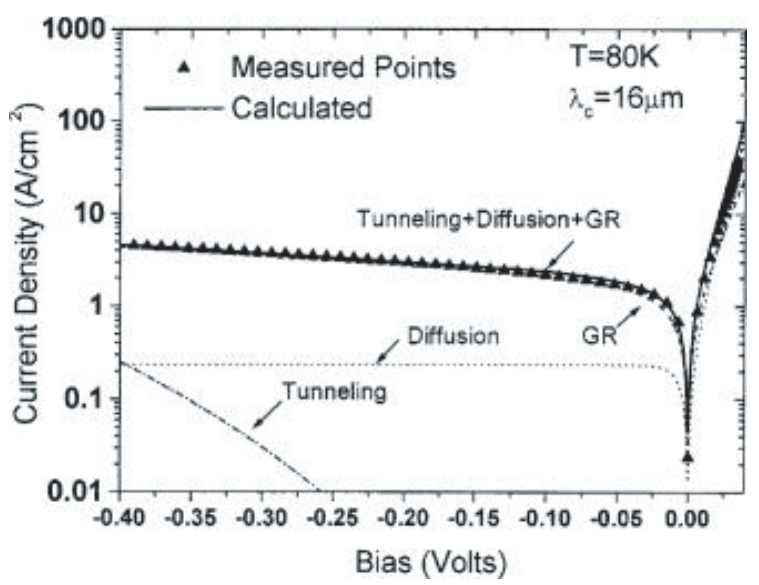

Fig. 9.17. The measured and modeled dark current density of a device with $\lambda_{c}=16 \mu \mathrm{m}$ at $T=80 \mathrm{~K}$. The model consists of the tunneling, generation recombination, and diffusion components of the dark current and indicates that generation recombination is the dominant source of the dark current around zero bias.

and diffusion current densities, shows good agreement to the measured values for forward and reverse biases [84].

Also, we could observe the Wannier-Stark oscillation in the Zener tunneling current of these devices. Figure 9.18 shows the differential resistance of the device versus reverse voltage bias. An oscillation with a peak-to-peak separation of about $29.9 \mathrm{mV}$ is clearly visible. The thickness of the depleted layer was extracted to be $d_{p}=120 \mathrm{~nm}$, and the background doping level to be $p=2.1 \times 10^{15} \mathrm{~cm}^{-3}$. This value shows an excellent agreement to the calculated background doping level based on the dark current modeling. This agreement and the fact that the background calculation based on the Wannier-Stark oscillation can be calculated without involvement of unknown parameters, indicate that the above value must be very close to the real background level.

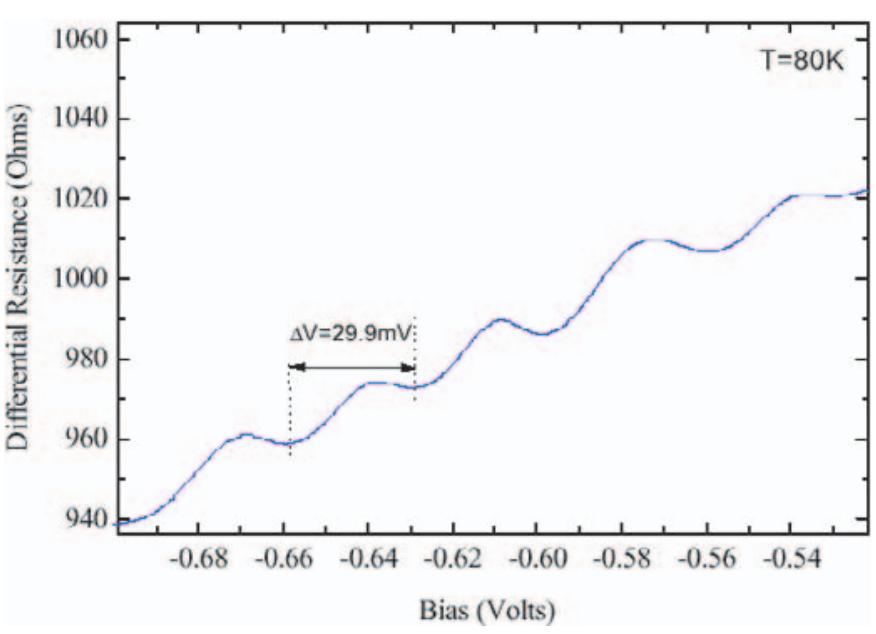

Fig. 9.18. The differential resistance of a device with $\lambda_{c}=$ $16 \mu \mathrm{m}$ at $T=80 \mathrm{~K}$ versus reverse bias shows a clear oscillation due to the Wannier-Stark oscillation in the Zener tunneling current.

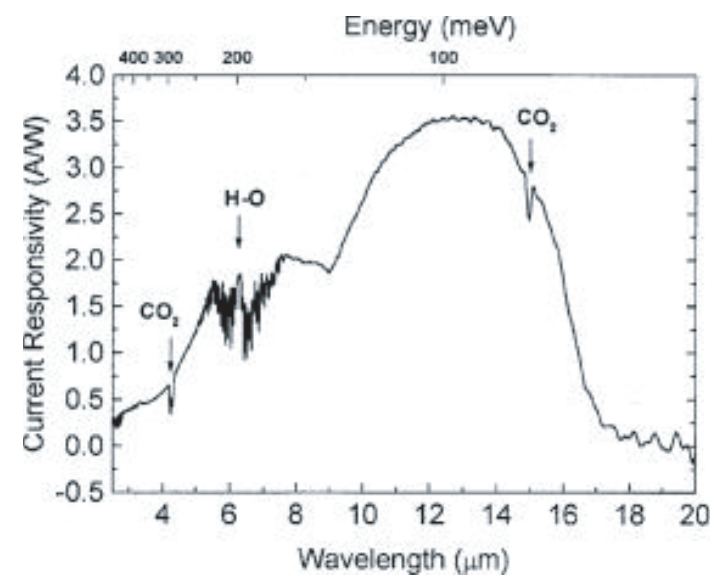

Fig. 9.19. Absolute spectral responsivity of a device with $\lambda_{c}=16 \mu \mathrm{m}$. The $90 \%$ to $10 \%$ cut-off energy width is about $12 \mathrm{meV}$. The absorption features of $\mathrm{CO}_{2}$ and $\mathrm{H}_{2} \mathrm{O}$ are due to the small difference in the optical path length of the background measurement and the detector measurement.

Absolute spectral responsivity was calculated from the measured spectral response of the device using FTIR spectroscopy system, and its photoresponse to a calibrated blackbody setup. Figure 9.19 shows the typical spectral responsivity of the detectors with $\lambda_{c}=16 \mu \mathrm{m}$. The absorption features of $\mathrm{CO}_{2}$ and $\mathrm{H}_{2} \mathrm{O}$ are due to the small difference in the optical path length of the background measurement and the detector measurement. The peak responsivity for the sample is about $3.5 \mathrm{~A} / \mathrm{W}$ which leads to a quantum efficiency of $\sim 35 \%$ at $12 \mu \mathrm{m}$. Adding antireflection coating can reduce the $30 \%$ surface reflection of the device and the quantum efficiency will reach to nearly $50 \%$. The $90 \%$ to $10 \%$ cutoff energy of the responsivity is only about $13 \mathrm{meV}$ at $T=80 \mathrm{~K}$ which is close to the thermal limit of $2 k T=12 \mathrm{meV}$. Such a sharp cutoff is a unique characteristic of our superlattices compared to the published results of other groups working on type-II 


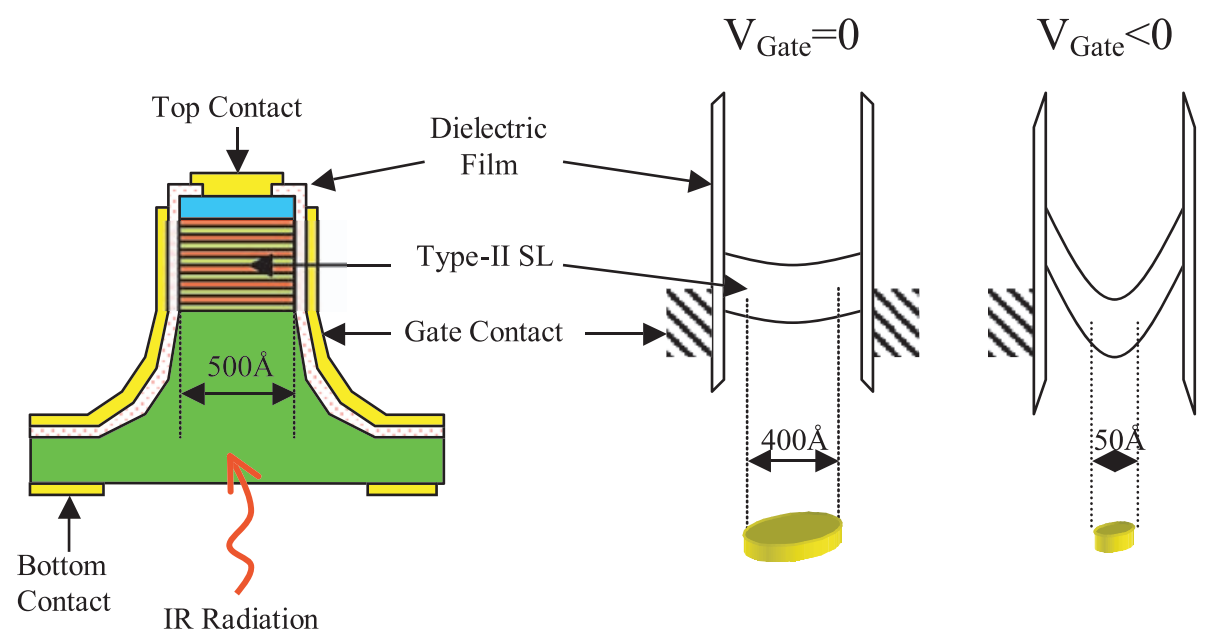

Fig. 9.20. Schematic diagram of the gated pillars with an adjustable lateral confinement.

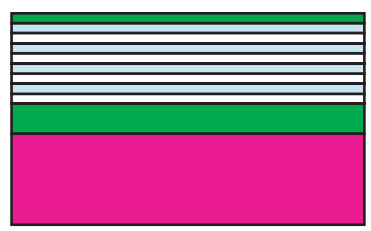

Starting material

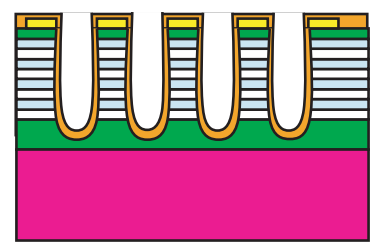

Oxide deposition

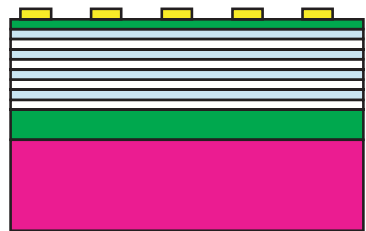

Top Contact definition

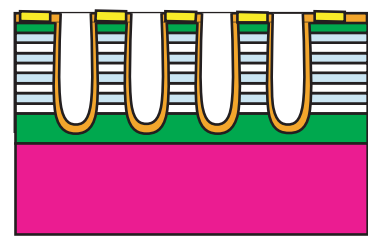

Top removal

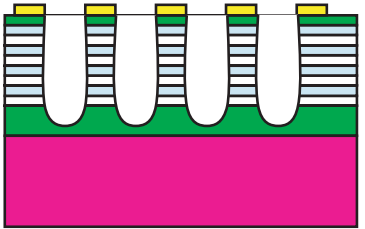

Dry etching

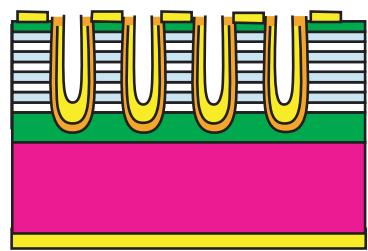

Gate Formation

Fig. 9.21. Realization of the pillars with advanced metallization and passivation techniques.

superlattice detectors [83-85] due to the use of binary materials, which are believed to have significantly enhanced the uniformity and reproducibility of the energy gap.

\subsection{Electrically induced adjustable 3-dimensional confinement}

Although Auger recombination rate could be decreased by engineering the band structure of the type-II superlattice, the Auger process is not completely eliminated.

By inducing quantum confinement in the $x$ and $y$ directions, the dispersion in these two directions can be quantized. This can eliminate the Auger recombination and hence the carrier lifetime will be limited by other mechanisms such as radiative and non-radiative recombination, which are several orders of magnitude slower than the Auger recombination at room temperature. Since the responsivity of the detector is proportional to the carrier lifetime, the responsitivity will also increase dramatically and will lead to a much higher detectivity.

Although Infra Red detector based on self-assembled quantum dots have already been demonstrated [86], the non-uniformity of the dot size leads to the line-width broadening and reduction of the signal. A method has been proposed which can produce high quality and highly uniform quantum dot structures. The starting material is a superlattice. Stacks of high quality quantum dots can be developed by etching pillars and consequent dielectric coating.

In order to achieve quantum size effect, the electrons need to be confined within tens of nanometers. However, surface leakage current will be a severe problem for such a small device, since the ratio of the surface to the volume increases dramatically.

In order to circumvent this problem, a gated device can be used as shown in Figure 9.20. Although the diameter of the device is 50 to $200 \mathrm{~nm}$, the effective conferment diameter can be adjusted by the gate voltage to a much smaller value. Moreover, the allowed energy states of the electrons can be changed by the gate voltage and hence the cutoff wavelength of the device. Figure 9.21 shows a realization scheme that can provide a large detector area containing a large number of closely packed pillars. 

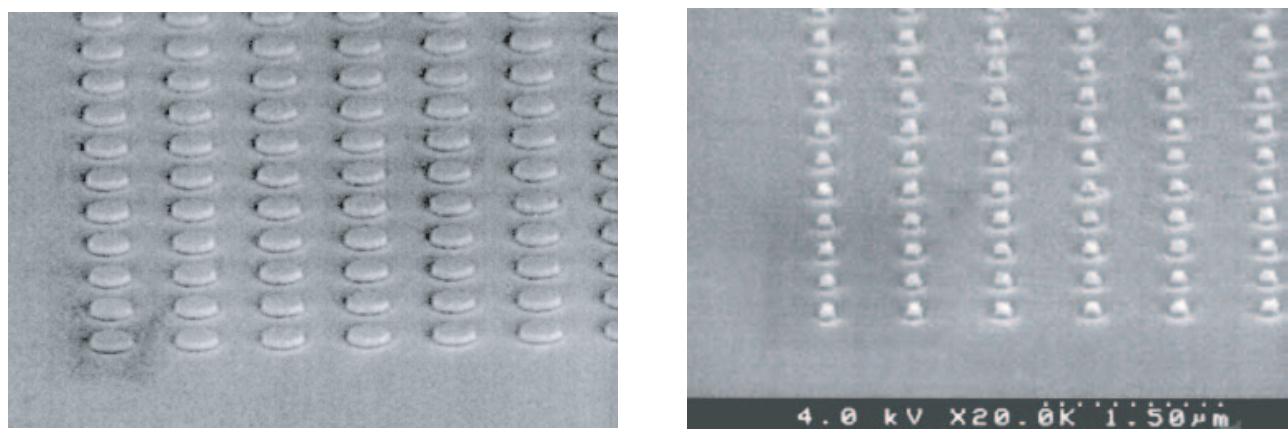

Fig. 9.22. Top metal contacts defined by e-beam lithography on the samples. (Left) $500 \mathrm{~nm}$ diameter contacts, and (right) $100 \mathrm{~nm}$ contacts.
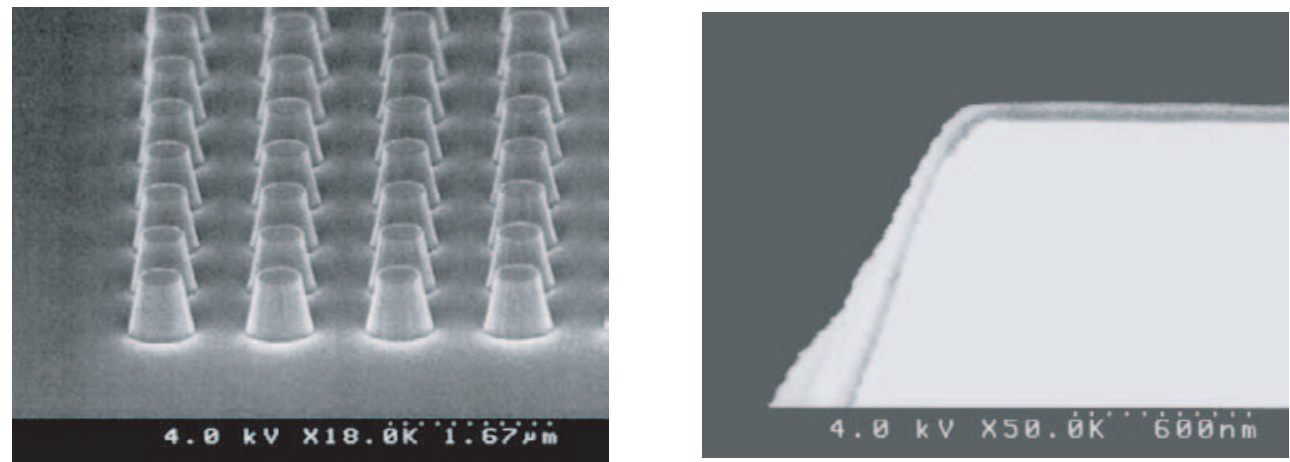

Fig. 9.23. (Left) Reactive ion etching was used for the formation of uniform two-dimensional arrays of pillars. (Right) SEM image of the cleaved edge of a mesa covered by a uniform layer of $\mathrm{Si}_{3} \mathrm{~N}_{4}$ with a thickness of about $50 \mathrm{~nm}$.

The feasibility of this approach has been demonstrated. Low energy e-beam lithography was used to produce top metal contacts. High quality metal contacts with diameter in the range of 1000 to $100 \mathrm{~nm}$ were successfully defined on the surface of the samples as shown in Figure 9.22 .

Reactive ion etching (RIE) was used to produce uniform anisotropic etching of the pillars through the GaSb test material. Figure 9.23 (Left) shows the SEM image of an array of $500 \mathrm{~nm}$ pillars produced by the dry etching. The vertical to horizontal etching is in excess of five. Twodimensional array of pillars with excellent uniformity over thousands of the square microns have been successfully produced.

Finally, passivation of the material was studied. Passivation is one of the most important steps for the realization of these quantum devices. The essential issues are the uniformity of the dielectric and coverage of the device surface. Plasma enhance chemical vapor deposition (PECVD) was used to form layers of silicon nitride on these structures. Figure 9.23 (Right) shows SEM image of a cleaved edge of a mesa covered with $50 \mathrm{~nm}$ silicon nitride layer.

\section{Conclusion}

The purpose of this review paper is to demonstrate the potential of Sb-based III-V semiconductors for the fabrication of optoelectronic devices in order to better under- stand the universe around us. Nature has provided numerous examples of efficient emission and detection systems, which have been and are still inspiring the development of optoelectronic devices that will enhance the human's abilities to better understand. For example, infrared detectors broaden our vision into the realm of heat, allowing remote sensing of an object's temperature. This has had a dramatic impact on how we perceive our environment, and has led to many types of thermal imaging, including night vision, infrared astronomy, medical diagnostics, and failure analysis. These newfound abilities have spurred the development of many new systems.

Sb-based materials are the basis for today's infrared optoelectronics. They have been widely used to produce high-speed electronic devices as well as optoelectronics devices such as emitters and detectors covering the wavelength range from mid infrared to long infrared and even very long infrared wavelength. The advanced epitaxy technologies of MOCVD and MBE enable the growth of high quality materials as well as novel structures. The development of nanotechnology has inspired the exploitation of quantum-sized effects for higher efficiency and added functionality.

The author would like to thank Yeun-ho Choi, Erick Michel, Jedon Kim, Jaejin Lee, Joseph Wojowski, Hooman Mohseni, Di Wu, Brett Lane, Yajun Wei, and especially Wei Zhang for their contribution to this work. The research at the Center for Quantum Devices and presented here was supported by the US 
Air Force (Gail Brown, William Mitchel, Daniel Johnstone), the US Army (Henry Everitt), the Office of Naval Research (Yoon-Soo Park) and the Defense Advanced Research Projects Agency (L.N. Durvasula, Ray Balcerak).

\section{References}

1. M. Schlessinger, Infrared Technology Fundamentals (Marcel Dekker, Inc., New York, 1995)

2. H. Walker, Z. Naturf. 7A, 744 (1952) (in German)

3. R.C. Sharma, Y.L. Ngai, Y.A. Chang, Binary alloy phase diagrams, ASM Int., Vol. 3, 2nd edn., edited by T.B. Massalski, p. 2286 (1990)

4. Y.J. Jung, M.K. Park, Si. Tae, K.H. Lee, H.J. Lee, J. Appl. Phys. 69, 3109 (1991)

5. M. Neuberger, III-V Ternary Semiconducting Compounds Data Tables (Plenum, New York, 1972)

6. W.J. Parrish, J.D. Balckwell, G.T. Kincaid, R.C. Paulson, Proc. SPIE 1540, 274 (1991)

7. A. Hoffman, D. Randall, Proc. SPIE 1540, 297 (1991)

8. R.D. Thom, T.L. Koch, J.D. Langan, W.J. Parrish, IEEE Trans. Electron. Dev. ED-27, 160 (1980)

9. Mortan International, "CVD" metalorganics for vapor phase epitaxy: product guide and literature review II

10. G.B. Stringfellow, Organometallic vapor-phase epitaxy: Theory and practice (Academic Press, 1989)

11. R.S. Wagner, Whiskers technology, edited by A.P. Levitt (Wiley, New York, 1970), p. 47

12. N.I. Buchan, C.A. Larsen, G.B. Stringfellow, J. Cryst. Growth 92, 591 (1988)

13. C.A. Larsen, S.H. Li, G.B. Stringfellow, Chem. Mat. 3, 39 (1991)

14. Y.H. Choi, R. Sudharsanan, C. Besikci, E. Bigan, M. Razeghi, Mat. Res. Soc. Symp. Proc. 281, 375 (1993)

15. C. Besikci, Y.H. Choi, R. Sudharsanan, M. Razeghi, J. Appl. Phys. 73, 5009 (1993)

16. J.R. Soderstrom, M.M. Cumming, J.-Y. Yao, T.G. Anderson, Semicond. Sci. Technol. 7, 337 (1992)

17. M. Yano, T. Takase, M. Kimata, Phys. Stat. Sol. A 54, $707(1979)$

18. A. Rose, Concept in photoconductivity and allied problems (Wiley, New York, 1963)

19. E. Michel, J.D. Kim, S. Javadpour, J. Xu, I. Ferguson, M. Razeghi, Appl. Phys. Lett. 69, 215 (1996)

20. I. Bloom, Y. Nemirovsky, IEEE Trans. Electron. Devices 40, 309 (1993)

21. H.H. Wieder, A.R. Clawson, Thin Solid Films 15, 217 (1973)

22. C.G. Bethea, B.F. Levine, M.Y. Yen, A.Y. Cho, Appl. Phys. Lett. 53, 291 (1988)

23. P.K. Chiang, S.M. Bedair, Appl. Phys. Lett. 53, 142 (988)

24. A. Rogalski, K. JoYwikowski, Infrared Phys. 29, 35 (1989)

25. M.Y. Yen, Appl. Phys. 64, 3306 (1988)

26. W.M. Coders, J.C. Woolley, Can. J. Phys. 46, 1207 (1968)

27. V.W.L. Chin, R.J. Egan, T.L. Tansley, J. Appl. Phys. 69, 3571 (1991)

28. C. Besikci, Y.H. Choi, G. Labeyrie, E. Bigan, M. Razeghi, J.B. Cohen, J. Carsello, V.P. Dravid, J. Appl. Phys. 76, $5820(1994)$

29. J.D. Kim, D. Wu, J. Wojkowski, J. Piotrowski, J. Xu, M. Razeghi, Appl. Phys. Lett. 68, 99 (1996)
30. J.D. Kim, S. Kim, D. Wu, J. Wojkowski, J. Xu, J. Piotrowski, E. Bigan, M. Razeghi, Appl. Phys. Lett. 67, 2645 (1995)

31. E. Michel, J.D. Kim, S. Park, J. Xu, I. Ferguson, M. Razeghi, Proc. SPIE 2685, 105 (1996)

32. M. Schilfgaarde, A. Sher, Appl. Phys. Lett. 62, 1857 (1993)

33. Y.H. Choi, C. Besikci, R. Sudharsanan, M. Razeghi, Appl. Phys. Lett. 63, 361 (1993)

34. K.T. Huang, R.M. Cohen, G.B. Stringfellow, J. Growth 156, 320 (1995)

35. N.K. Karam, R. Sudharsanan, T. Pardos, M.A. Dodd, J. Electron Mater. 25, 1209 (1996)

36. Y.H. Choi, P.T. Staveteig, E. Bigan, M. Razeghi, J. Appl. Phys. 75, 3196 (1994)

37. P.T. Staveteig, Y.H. Choi, G. Labeyrie, E. Bigan, M. Razeghi, Appl. Phys. Lett. 64, 460 (1994)

38. E. Bigan, Y.H. Choi, G. Labeyrie, M. Razeghi, Proc. SPIE 2145, 2 (1994)

39. M. Razeghi, Y.H. Choi, J.D. Kim, E, Bigan, Proc. Electrochem. Soc., Miami Beach, Florida, October, 1994

40. M. Razeghi, Y.H. Choi, X. He, C.J. Sun, Mat. Sci. Technol. 11, 3 (1995)

41. J.C. Phillips, Bonds and Bands in Semiconductors (Academic, New York, 1973)

42. J.J. Lee, M. Razeghi, Appl. Phys. Lett. 76, 297 (2000)

43. C. Besikci, Y.H. Choi, G. Labeyrie, E. Bigan, M. Razeghi, J.B. Cohen, J. Carsello, V.P. Dravid, J. Appl. Phys. 76, 5820 (1994)

44. V.W. Chin, R.J. Egan, T.L. Tansley, J. Appl. Phys. 69, 3571 (1991)

45. J.W. Harrison, J.R. Hauser, J. Appl. Phys. 47, 292 (1976)

46. J.J. Lee, J.D. Kim, M. Razeghi, Appl. Phys. Lett. 71, 2298 (1997)

47. J.J. Lee, J.D. Kim, M. Razeghi, Appl. Phys. Lett. 73, 602 (1998)

48. O. Duchemin, III-V Materials (1994)

49. T. Fukui, Y. Horikoshi, Jpn J. Appl. Phys. 19, L53 (1980)

50. K. Onabe, NEC Res. Dev. 72, 1 (1984)

51. Landolt and Börnstein, Numerical Data and Functional Relationships in Science and Technology: Semiconductors (Springer-Verlag, Berlin, 1982)

52. J. Diaz, H. Yi, A. Rybaltowski, B. Lane, G. Lukas, D. Wu, S. Kim, M. Erdtmann, E. Kaas, M. Razeghi, Appl. Phys. Lett. 70, 40 (1997)

53. J.W. Matthews, A.E. Blakeslee, J. Cryst. Growth 27, 118 (1974)

54. R. People, J.C. Bean, Appl. Phys. Lett. 47, 322 (1985)

55. H. Mohseni, V. Litvinov, M. Razeghi, Phys. Rev. B 58, 378 (1998)

56. A. Rybaltowski, Y. Xiao, D. Wu, B. Lane, H. Yi, H. Feng, J. Diaz, M. Razeghi, Appl. Phys. Lett. 71, 2430 (1997)

57. G.A. Sai-Halasz, R. Tsu, L. Esaki, Appl. Phys. Lett. 30, 651 (1977)

58. G.A. Sai-Halasz, L.L. Chang, J.M. Welter, L. Esaki, Solid State Commun. 27, 935 (1978)

59. G.A. Sai-Halasz, L. Esaki, Phys. Rev. B 18, 2812 (1978)

60. D.L. Smith, C. Mailhiot, J. Appl. Phys. 62, 2545 (1987)

61. R. Youngdale, J.R. Meyer, C.A. Hoffman, F.J. Bartoli, Appl. Phys. Lett. 64, 3160 (1994)

62. H. Xie, W.I. Wang, J. Appl. Phys. 76, 92 (1994)

63. J.L. Johnson, L.A. Samoska, A.C. Gossard, J. Merz, M.D. Jack, G.R. Chapman, B.A. Baumgratz, K. Kosai, S.M. Johnson, J. Appl. Phys. 80, 1116 (1996) 
64. F. Fuchs, U. Weimer, W. Pletschen, J. Schmitz, E. Ahlswede, M. Walther, J. Wagner, P. Koidl, Appl. Phys. Lett. 71, 3251 (1997)

65. B.H. Yang, D. Zhang, R.Q. Yang, C.-H. Lin, S.J. Murry, S.S. Pei, Appl. Phys. Lett. 72, 2220 (1998)

66. C.L. Felix, J.R. Meyer, I. Vurgaftman, C.H. Lin, S.J. Murry, D. Zhang, S.S. Pei, Phot. Tech. Lett. 9, 734 (1997)

67. H. Mohseni, E. Michel, J. Sandoen, M. Razeghi, W. Mitchel, G. Brown, Appl. Phys. Lett. 71, 1403 (1997)

68. N. Herres, F. Fuchs, J. Schmitz, K. Pavlov, J. Wagner, J. Ralston, P. Koidl, C. Gadaleta, G. Scamarcio, Phys. Rev. B 53, 15688 (1996)

69. H. Mohseni, E.J. Michel, M. Razeghi, W.C. Mitchel, G.J. Brown, SPIE 3287, 30 (1998)

70. E.V. Kutchis, Galvanomagnetic Effects and Methods of Their Investigations (Izd. Radiosvyaz, Moscow, 1990)

71. G. Zegrya, A. Andreev, Appl. Phys. Lett. 67, 2681 (1995)

72. J. Piotrowski, W. Galus, M. Grudzien, Infrared Phys. 31, 1 (1990)

73. D. Wang, G. Bosman, Y. Wang, S. Li, J. Appl. Phys. 77, 1107 (1995)

74. D. Long, Infrared Phys. 7, 169 (1967)

75. S. Slivken, A. Matlis, C. Jelen, A. Rybaltowski, J. Diaz, M. Razeghi, Appl. Phys. Lett. 74, 173 (1999)
76. H. Mohseni, M. Razeghi, Proc. 1999-ISDRS, 563 (1999)

77. H. Mohseni, J. Wojkowski, M. Razeghi, IEEE J. Quantum Elect. 35, 1041 (1999)

78. D. Chow, R. Miles, J. Schulman, D. Collins, T. McGill, Semicond. Sci. Technol. 6, C47 (1991)

79. H. Mohseni, E. Michel, J. Sandven, M. Razeghi, W. Mitchel, G. Brown, Appl. Phys. Lett. 71, 1403 (1997)

80. H. Mohseni, A. Tahraoui, J. Wojkowski, M. Razeghi, G.J. Brown, W.C. Mitchel, Y.S. Park, Appl. Phys. Lett. 77, $1572(2000)$

81. S. Slivken, A. Matlis, C. Jelen, A. Rybaltowski, J. Diaz, M. Razeghi, Appl. Phys. Lett. 74, 173 (1999)

82. H. Mohseni, M. Razeghi, G.J. Brown, Y.S. Park, Appl. Phys. Lett. 78, 2107 (2001)

83. M. Young, D. Chow, A, Hunter, R. Miles, Appl. Surf. Sci. 123/124, 395 (1998)

84. F. Fuchs, U. Weimar, E. Ahlswede, W. Pletschen, J. Schmitz, M. Walther, Proc. SPIE 3287, 14 (1998)

85. R. Miles, J. Wilson, Proc. SPIE 2816, 76 (1996)

86. S. Kim, H. Mohseni, M. Erdtmann, E. Michel, C. Jalen, M. Razeghi, Appl. Phys. Lett. 73, 963 (1998)

To access this journal online: www.edpsciences.org 